\title{
A study of the ${ }^{16} \mathrm{O}\left(\mathrm{e}, \mathrm{e}^{\prime} \mathrm{p}\right)$ reaction at deep missing energies
}

by

Nilanga Kumara Bamunusinha Liyanage

B.S., Massachusetts Institute of Technology (1993)

Submitted to the Department of Physics

in partial fulfillment of the requirements for the degree of

Doctor of Philosophy in Physics

at the

\section{MASSACHUSETTS INSTITUTE OF TECHNOLOGY}

February 1999

(C) Massachusetts Institute of Technology 1999. All rights reserved.

Signature of Author

Department of Physics

February 22, 1999

Certified by

Professor William Bertozzi

Thesis Supervisor

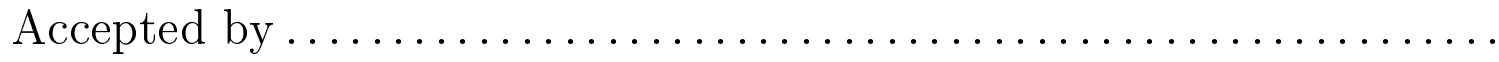

Prof. Thomas J. Greytak Associate Department Head for Education 


\title{
A study of the ${ }^{16} \mathrm{O}\left(\mathrm{e}, \mathrm{e}^{\prime} \mathbf{p}\right)$ reaction at deep missing energies
}

\author{
by \\ Nilanga Kumara Bamunusinha Liyanage \\ Submitted to the Department of Physics \\ on February 22, 1999, in partial fulfillment of the \\ requirements for the degree of \\ Doctor of Philosophy in Physics
}

\begin{abstract}
The ${ }^{16} \mathrm{O}\left(\mathrm{e}, \mathrm{e}^{\prime} \mathrm{p}\right)$ reaction was studied in the first physics experiment performed at Jefferson lab Hall A. In the quasielastic region cross sections were measured for both quasi-parallel and perpendicular kinematics at $q=1000 \mathrm{MeV}$ and $\omega=445 \mathrm{MeV}$. From the data acquired in quasi-parallel kinematics, longitudinal and transverse response functions, $R_{L}$ and $R_{T}$ were separated for $E_{\text {miss }}<60 \mathrm{MeV}$. The perpendicular kinematics data were used to extract $R_{L T}, R_{T}$, and $R_{L}+\frac{V_{T T}}{V_{L}} R_{T T}$ response functions for the same $E_{\text {miss }}$ range and for $P_{\text {miss }}<310 \mathrm{MeV} / \mathrm{c}$. The ${ }^{16} \mathrm{O}\left(\mathrm{e}, \mathrm{e}^{\prime} \mathrm{p}\right)$ cross section was measured in the dip region at $q=1026 \mathrm{MeV}$ and $\omega=586 \mathrm{MeV}$ for $10 \mathrm{MeV}<E_{\text {miss }}<320 \mathrm{MeV}$. This thesis presents the results for the missing energy continuum $\left(E_{\text {miss }}>25 \mathrm{MeV}\right)$ from this experiment.

The measured cross sections and the response functions are compared to DWIA and HF-RPA calculations of the strength for the single particle knockout from the $1 \mathrm{~s}_{1 / 2}$ shell. For the quasielastic, quasi-parallel kinematics setting, the measured cross sections and the response functions for $E_{\text {miss }}<50 \mathrm{MeV}$ are mostly accounted for by the single particle knockout from $1 \mathrm{~s}_{1 / 2}$. For $50 \mathrm{MeV}<E_{\text {miss }}<60 \mathrm{MeV}, R_{T}$ remains strong while $R_{L}$ appears to go to zero. The difference between the longitudinal and transverse spectral functions, $S_{T}-S_{L}$, which is expected to be zero for a free nucleon, is close to zero at lower $E_{\text {miss }}$ but increases above the two nucleon emission threshold.

The behavior of $R_{L}$ and $R_{T}$ for the $\left|\theta_{p q}\right|=8^{\circ}$ setting is very similar to that of the quasi-parallel setting: for $50 \mathrm{MeV}<E_{\text {miss }}<60 \mathrm{MeV}, R_{L} \rightarrow 0$ while $R_{T}$ remains strong. However $R_{L T}$ is non-zero for $50 \mathrm{MeV}<E_{\text {miss }}<60 \mathrm{MeV}$, indicating that $R_{L}$ might be small but non-zero in this $E_{\text {miss }}$ range.

For the quasielastic, $\left|\theta_{p q}\right|=16^{\circ}$ setting, the relative strength in the continuum compared to the strength in the valence states is much higher than at lower $P_{m i s s}$ settings. Only a small fraction of this continuum strength is accounted for by the calculated $1 \mathrm{~s}_{1 / 2}$ knockout strength. $R_{T}$ and $R_{L T}$ response functions are also much stronger than the prediction, while the longitudinal response is small as predicted.

The cross sections calculated for the quasielastic, $\left|\theta_{p q}\right|=20^{\circ}$ settings show even higher relative strengths in the continuum compared to the valence states. The phase space of the $\theta_{p q}=-20^{\circ}$ setting extends across the two body correlated ridge and shows an increase in cross section around the location of the ridge.

The cross section measured in the dip region exhibits continuum strength up to the highest measured $E_{m i s s}$ of $320 \mathrm{MeV}$. An increase in this cross section is observed above the pion production threshold.
\end{abstract}

Thesis Supervisor: William Bertozzi

Title: Professor of Physics 
In memory of my Father

Sirisena Bamunusinha Liyanage

1930-1982 


\section{Contents}

1 Introduction $\quad 11$

1.1 Electron scattering . . . . . . . . . . . . . 11

1.2 Inclusive electron scattering f . . . . . . . . . . . 12

1.3 Exclusive $\left(\mathrm{e}, \mathrm{e}^{\prime} \mathrm{p}\right)$ scattering . . . . . . . . . . . 15

1.3.1 The $\left(\mathrm{e}, \mathrm{e}^{\prime} \mathrm{p}\right)$ reaction formalism . . . . . . . . . 18

1.4 Plane Wave Impulse Approximation . . . . . . . . . . . . . . 22

1.5 Distorted Wave Impulse Approximation . . . . . . . . . . . 23

1.6 Previous $\left(\mathrm{e}, \mathrm{e}^{\prime} \mathrm{p}\right)$ measurements in the $E_{\text {miss }}$ continuum . . . . . . 24

1.7 The present experiment . . . . . . . . . . . . 32

2 The Experiment $\quad 34$

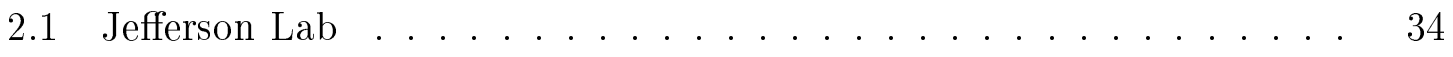

2.2 Hall A . . . . . . . . . . . . . . . 36

2.2.1 Beam line equipment . . . . . . . . . . 37

2.2.2 Waterfall target . . . . . . . . . . 39

2.2.3 High Resolution Spectrometer (HRS) pair . . . . . . . . 41

2.2.4 HRS detector packages . . . . . . . . . . . . 42

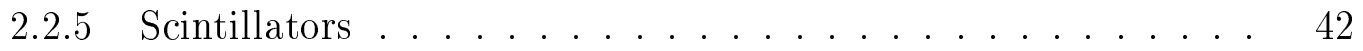

2.2.6 Gas Cherenkov counter ............... 43

2.2.7 Vertical Drift Chambers .............. 44

2.2.8 Trigger electronics . . . . . . . . . . . 52

2.2.9 Hall A data acquisition system . . . . . . . . 55

2.3 Kinematics and experimental method . . . . . . . . . 57 
2.3.1 Quasielastic region . . . . . . . . . . . . 57

2.3.2 Dip region kinematics $\ldots \ldots \ldots \ldots$. . . . . . 60

3 Data Analysis $\quad 62$

3.1 Analysis of the raw data . . . . . . . . . . . . 62

3.2 Beam energy measurement . . . . . . . . . . . . . . 66

3.3 Coincidence Time-of-Flight (TOF) calculation . . . . . . . . . 68

3.4 Pion Rejection . . . . . . . . . . . . . . . . . . . . . 71

3.5 Events with collimator punch-through protons . . . . . . . 76

3.5.1 Estimation of punch-through contamination . . . . . . . 79

3.6 Spectrometer normalization $\ldots \ldots \ldots \ldots$

3.6.1 Waterfall target thickness measurement . . . . . . . . . . 82

3.6.2 $\mathrm{H}\left(\mathrm{e}, \mathrm{e}^{\prime}\right)$ cross section calculation . . . . . . . . . . 84

3.6.3 Normalization of $\mathrm{H}\left(\mathrm{e}, \mathrm{e}^{\prime}\right)$ cross section to world data . . . . 86

3.6.4 Hadron spectrometer trigger efficiency determination using $\mathrm{H}\left(\mathrm{e}, \mathrm{e}^{\prime} \mathrm{p}\right) \quad 88$

3.6.5 Focal plane relative efficiency $\ldots \ldots \ldots \ldots$

4 Cross section and beyond $\quad 94$

4.1 Cross section calculation . . . . . . . . . . . . . . . . . 94

4.1 .1 Luminosity calculation . . . . . . . . . . . . . . 95

4.1 .2 Phase-space volume calculation $\ldots \ldots \ldots$. . . . . 97

4.1 .3 Dead time correction . . . . . . . . . . . . . . . 97

4.2 Radiative Corrections . . . . . . . . . . . . . . . . . . 98

4.2 .1 Internal radiation $\ldots \ldots \ldots \ldots \ldots$

4.2 .2 External radiation . . . . . . . . . . . . . . . 102

4.2.3 Radiative unfolding procedure for $\left(\mathrm{e}, \mathrm{e}^{\prime} \mathrm{p}\right)$ experiments . . . . 104

4.3 Cross section calculation procedure . . . . . . . . . . . 110

4.4 Response function calculation . . . . . . . . . . . . . . . . . . 112

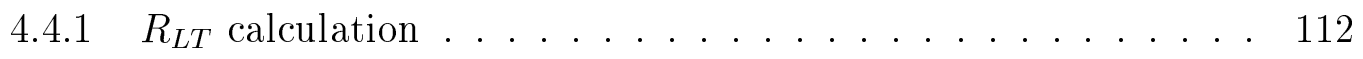

$4.4 .2 R_{L}+\frac{V_{T T}}{V_{L}} R_{T T}, R_{T}$ separation $\ldots \ldots \ldots \ldots \ldots \ldots$

4.5 Systematic uncertainty . . . . . . . . . . . . . . . 115 
5 Results and discussion $\quad 118$

5.1 Dip region results . . . . . . . . . . . . . . . . . . . . . . . . . 119

5.1.1 10.0 MeV $<E_{\text {miss }}<60.0 \mathrm{MeV}$ : single particle knockout of $1 \mathrm{p}_{1 / 2}, 1 \mathrm{p}_{3 / 2}$ and $1 \mathrm{~s}_{1 / 2}$ protons $\ldots \ldots \ldots \ldots \ldots$

5.1.2 28.0 MeV $<E_{\text {miss }}<150.0 \mathrm{MeV}$ : multi-nucleon knockout $\ldots \quad$. 125

5.1.3 $150.0 \mathrm{MeV}<E_{\text {miss }}<320.0 \mathrm{MeV}$ : pion production from ${ }^{16} \mathrm{O}$ in the target and multi-nucleon knockout $\ldots \ldots \ldots \ldots$

5.2 Quasielastic region results $(|\vec{q}|=1 \mathrm{GeV}, \omega=445 \mathrm{MeV}) \ldots \ldots .128$

5.2.1 Quasi-parallel kinematics (Quasielastic kinematics: $|\vec{q}|=1$ $\mathrm{GeV}, \omega=445 \mathrm{MeV}) \ldots \ldots \ldots \ldots \ldots$

5.2.2 Perpendicular kinematic settings (Quasielastic kinematics: $|\vec{q}|=$ $1 \mathrm{GeV}, \omega=445 \mathrm{MeV}) \ldots \ldots \ldots \ldots . \ldots \ldots$

5.3 Summary . . . . . . . . . . . . . . . 160

5.4 Conclusion . . . . . . . . . . . . . . . . . . . . . . . . 162

A Calibration of the Hall A High Resolution Spectrometer pair $\quad 164$

A.1 Coordinate systems . . . . . . . . . . . . . . . . . . . . 164

A.2 Approach . . . . . . . . . . . . . . . . . . . . 169

A.3 Experimental procedure . . . . . . . . . . . . . . 173

B Basic HRS tensor elements used for E-89003 175

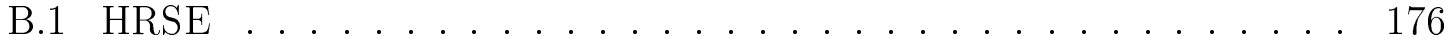

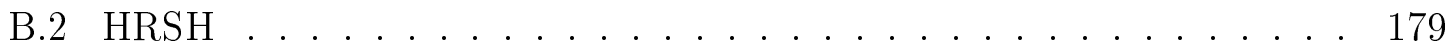

C The trigger electronics layout 182 


\section{Chapter 1}

\section{Introduction}

This thesis presents high missing energy results from an ${ }^{16} \mathrm{O}\left(\mathrm{e}, \mathrm{e}^{\prime} \mathrm{p}\right)$ experiment performed in experimental Hall A at the Thomas Jefferson National Accelerator Facility. The results for the valence states from the same experiment are reported elsewhere [1].

This thesis is organized as follows. The first part of this chapter discusses inclusive electron scattering while the latter part presents the $\left(e, e^{\prime} p\right)$ reaction formalism and a short discussion of previous (e,e'p) experiments that motivated the present experiment. Chapter 2 gives a detailed description of the experimental setup. In chapters 3 and 4, a discussion of the data analysis and the extraction of the response functions is given. Finally, chapter 5 presents and discusses the results of the experiment.

\subsection{Electron scattering}

Electron scattering is one of the most powerful tools used in the exploration of nuclear matter. The main strengths of electron scattering are that the electromagnetic interaction is calculable with well understood Quantum Electro-Dynamics (QED), and it is relatively weak compared to the hadronic interaction, which allows the electromagnetic interaction to be described with the one photon exchange approximation. The weakness of the electro-magnetic interaction also means that the resulting virtual photon can probe the entire nuclear volume, in contrast to hadronic probes that mostly sample the nuclear surface.

The following sections present a brief description of electron scattering including 
a discussion of some of the previous experiments that have motivated the present experiment.

\subsection{Inclusive electron scattering}

Fig. 1-1 presents an illustration of the energy spectrum for electrons scattered from nuclei in single arm $\left(\mathrm{e}, \mathrm{e}^{\prime}\right)$ experiments. The horizontal axis of the plot gives the electron energy transfer $\omega$ while the vertical axis gives the cross section. At $\omega=\frac{Q^{2}}{2 A}$ (where $A$ is the nuclear mass and $Q^{2}$ is the square of the four momentum of the virtual photon) the scattering is elastic, resulting in a sharp peak at the lowest edge of the spectrum. At slightly higher values of $\omega$, the target nucleus is excited to higher energy bound states. These show up as a series of discrete peaks in the spectrum. The broad bump just above these peaks corresponds to the collective modes of excitation of the nucleus and is referred to as the Giant Resonance region.

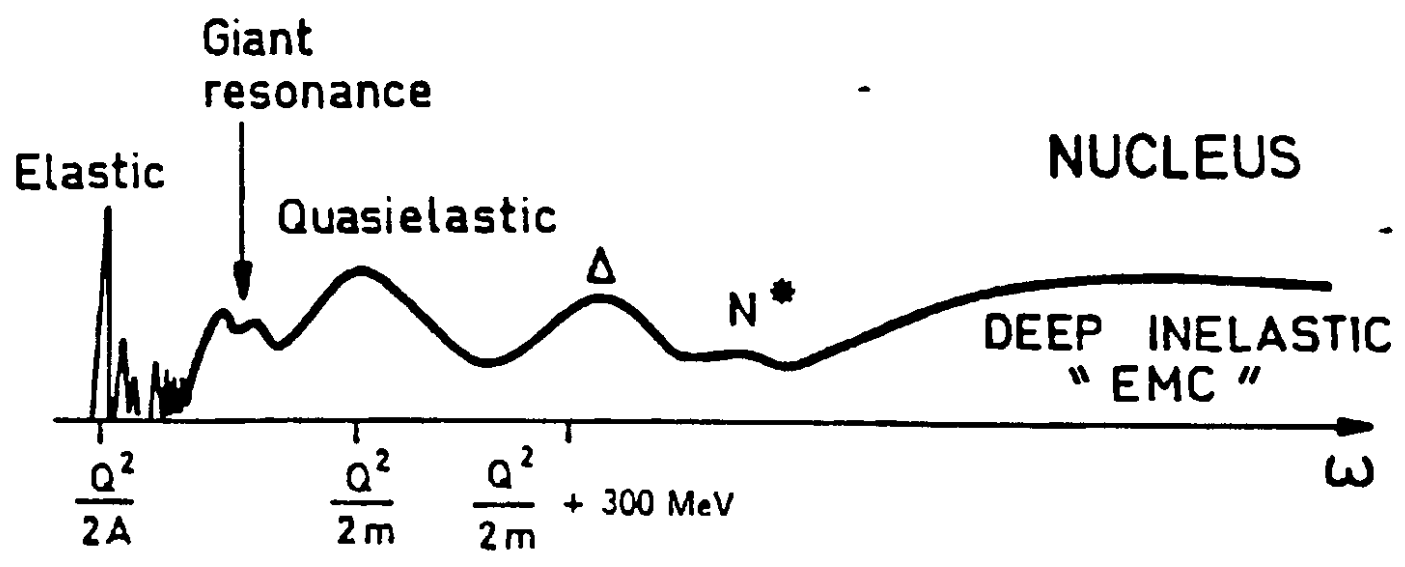

Figure 1-1: A generic energy spectrum for $\left(\mathrm{e}, \mathrm{e}^{\prime}\right)$ at a fixed value of $Q^{2}=\vec{q}^{2}-\omega^{2}$.

The quasielastic peak is centered near $\omega=\frac{Q^{2}}{2 m}$ (where $m$ is the nucleon mass). Note that $\omega=\frac{Q^{2}}{2 m}$ is the kinematic condition which corresponds to elastic scattering from a free nucleon. After the quasielastic peak come two broad bumps which correspond to the $\Delta$ and $\mathrm{N}^{\star}$ resonances. At these resonances, the knockout of a nucleon is coupled to an excitation of the nucleon. The region between the quasielastic peak and the $\Delta$-resonance peak is called the dip region. At yet higher values of $\omega$, the cross section increases to the deep-inelastic plateau, which corresponds to electrons scattering off 
the constituents of the nucleon.

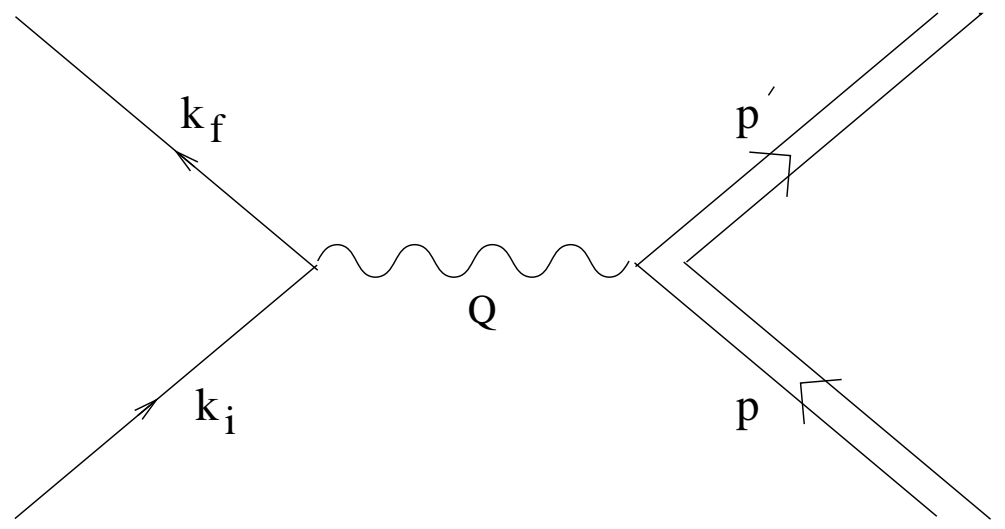

Figure 1-2: Feynman diagram for $\left(e, e^{\prime}\right)$ in the one photon exchange approximation.

For light nuclei with $Z \alpha \ll 1$ (where $Z$ is the nuclear charge and $\alpha$ is the fine structure constant), the scattering of the electron can be approximated by the exchange of a single virtual photon as illustrated in Fig. 1-2. In a simple model known as the Impulse Approximation (IA), the nucleus can be treated as a collection of non-interacting nucleons. In the IA, the virtual photon can be thought of as being absorbed by a single nucleon without disturbing the rest of the nucleus.

In this simple picture, the quasielastic bump represents the knock-out of a single nucleon by an electron. To first approximation, the width of the quasielastic peak can be attributed to the momentum distribution of nucleons inside the nucleus. Moniz [2] has shown that a Fermi gas model (where the nucleus is represented by a box of finite size in which nucleons move independently) can describe the widths of the quasielastic peaks measured by Zimmerman [3] for several nuclei. The results of Whitney [4] also showed agreement between the Fermi gas model predictions and the measured quasielastic peak widths for a range of nuclei from ${ }^{6} \mathrm{Li}$ to ${ }^{208} \mathrm{~Pb}$.

Despite these successes of the Fermi gas model, serious problems surfaced when separation of longitudinal and transverse components of the cross section was performed using the so-called Rosenbluth technique. In the one photon exchange approximation the quasielastic inclusive cross section can be written as 


$$
\frac{d^{3} \sigma}{d E^{\prime} d \Omega_{e}}=\sigma_{M}\left[\frac{Q^{4}}{\vec{q}^{4}} R_{L}\left(Q^{2}, \omega\right)+\left(\frac{Q^{2}}{2 \vec{q}^{2}}+\tan ^{2}\left(\frac{\theta_{\mathrm{e}}}{2}\right)\right) R_{T}\left(Q^{2}, \omega\right)\right],
$$

where $\theta_{e}$ is the electron scattering angle and $\sigma_{M}$ is the Mott cross section, given by

$$
\sigma_{M}=\frac{\alpha^{2} \cos ^{2}\left(\theta_{\mathrm{e}} / 2\right)}{4 E_{0}^{2} \sin ^{2}\left(\theta_{\mathrm{e}} / 2\right)}
$$

with $E_{0}$ being the electron beam energy and $\alpha$ the fine structure constant. $R_{L}$ and $R_{T}$ are two independent response functions that describe the electromagnetic properties of the hadronic system. The terms longitudinal and transverse refer to the couplings of the hadronic current to the longitudinal and transverse polarization components of the virtual photon. It is important to note that $R_{L}$ and $R_{T}$ are functions of only $Q^{2}$ and $\omega$. Therefore, by measuring the cross section at two different kinematic settings with the same values of $Q^{2}$ and $\omega$, the $R_{L}$ and $R_{T}$ contributions to the cross section can be separated.

For quasielastic kinematics, Rosenbluth separations [5], [6], [7], for nuclei with $A \geq 4$ have shown that while the transverse response is reproduced fairly well, the longitudinal response is suppressed by as much as $40 \%$ compared to the impulse approximation predictions. As a result, and perhaps more importantly, the ratio $\frac{R_{T}}{R_{L}}$ is enhanced with respect to the quasi-free impulse approximation. Rosenbluth separations performed in the dip region have demonstrated much more transverse strength than can be explained theoretically by pion photoproduction and meson exchange currents. Therefore, in both quasielastic and dip kinematics, $\frac{R_{T}}{R_{L}}$ has shown unexplained enhancement compared to the free proton.

There have been many theoretical attempts to explain this $\frac{R_{T}}{R_{L}}$ enhancement [8], [9], [10], [11], [12], [13], [14], [15], [16], [17], [18]. Many of these calculations have been able to achieve a reduction in the longitudinal responses sufficient to approximately reproduce the experimental results. However, these calculations have resulted in unacceptably bad fits to the measured transverse responses. More recently, a considerable amount of work has been invested in developing a Relativistic Fermi Gas model (RFG) to describe the behavior of quasielastic scattering [19], [20], [21]. 
A reanalysis of the world quasielastic scattering data for ${ }^{56} \mathrm{Fe}$ and ${ }^{40} \mathrm{Ca}$ has been performed recently by Jourdan [22]. This analysis has indicated that there is no reduction of the longitudinal strength compared to the prediction of the Fermi gas model.

It should also be noted that a recent measurement of ${ }^{40} \mathrm{Ca}\left(\mathrm{e}, \mathrm{e}^{\prime}\right)$ at MIT-Bates by Yates et al. [23], [24] has yielded substantially larger longitudinal responses than those obtained previously. The integrated longitudinal strength from this experiment agrees with the RFG prediction to within $\pm 18 \%$, whereas previous data indicate as much as a $40 \%$ reduction in the longitudinal strength compared to the RFG prediction.

\subsection{Exclusive (e,e'p) scattering}

Since the inclusive cross section consists of many exclusive channels, it has been inherently difficult to study and evaluate the effects of the different channels on the $\frac{R_{T}}{R_{L}}$ ratio using inclusive $\left(e, e^{\prime}\right)$ scattering experiments. Semi-exclusive $\left(e, e^{\prime} p\right)$ experiments, on the other hand, provide further insight into the understanding of these anomalies. In exclusive experiments, the contributions from different valence states and the continuum channels can be disentangled. Further, the longitudinal and transverse responses for these different channels may be studied in the hope of understanding the source of the inclusive $\frac{R_{T}}{R_{L}}$ enhancement. 


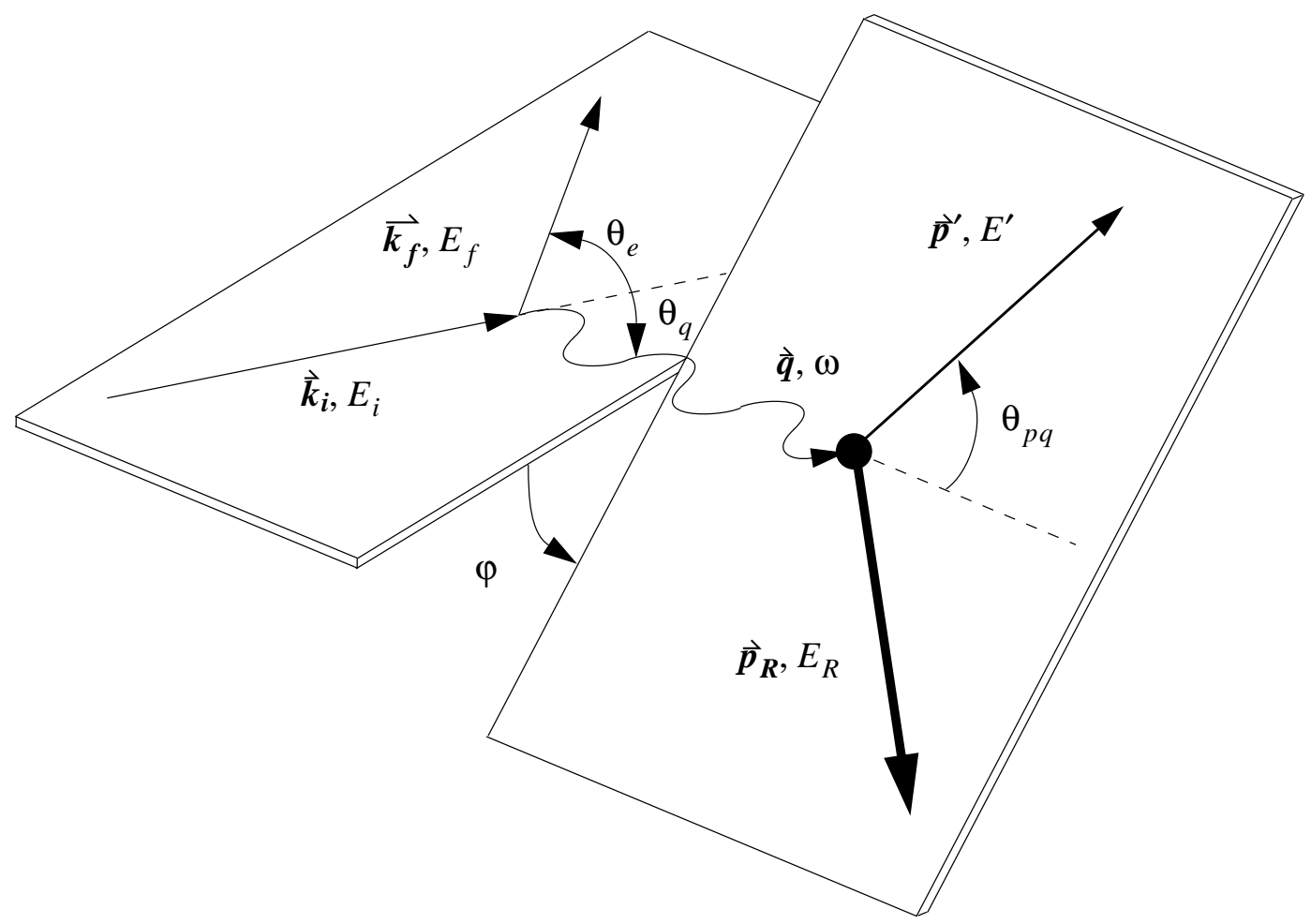

Figure 1-3: Kinematics for the $\left(\mathrm{e}, \mathrm{e}^{\prime} \mathrm{p}\right)$ reaction. The out-of-plane angle $\phi$ is defined to be the angle between the reaction plane and the scattering plane. For the present experiment, $\phi$ angles of $0^{\circ}$ and $180^{\circ}$ were used.

The kinematics for the (e,e'p) reaction is shown in Fig. 1-3. On the electron side of the diagram, the incident electron four vector is denoted by $k_{i}^{\mu}=\left(E_{i}, \vec{k}_{i}\right)$ and the scattered electron by $k_{f}^{\mu}=\left(E_{f}, \vec{k}_{f}\right)$. For the ultra-relativistic case, the electron mass can be neglected so that $\left|\vec{k}_{i}\right|=E_{i}$ and $\left|\vec{k}_{f}\right|=E_{f}$. The two vectors $\vec{k}_{i}$ and $\vec{k}_{f}$ define the scattering plane. The difference between the initial and final electron four momenta is the momentum transfered to the target nucleus, $Q=(\omega, \vec{q})$ :

$$
\begin{aligned}
Q & =k_{i}^{\mu}-k_{f}^{\mu} \\
\omega & =E_{i}-E_{f} \\
\vec{q} & =\vec{k}_{i}-\vec{k}_{f} .
\end{aligned}
$$

The four momentum of the target nucleus is defined to be $P_{A}^{\mu}=\left(E_{A}, \vec{P}_{A}\right)$. The target is at rest in the lab frame so that $\vec{P}_{A}=0$ and $E_{A}$ is equal to the mass of the target. The final proton has four momentum $P^{\prime \mu}=\left(E^{\prime}, \vec{p}^{\prime}\right)$, and the recoiling nucleus has 
four momentum $P_{R}^{\mu}=\left(E_{R}, \vec{p}_{R}\right)$. The conservation of energy and three momentum yields

$$
\begin{aligned}
\omega+M_{A} & =E^{\prime}+E_{R} \\
& =M_{p}+T_{p}+M_{B}+T_{R}, \text { and } \\
\vec{q} & =\vec{p}^{\prime}+\vec{p}_{R},
\end{aligned}
$$

where $M_{p}$ is the mass of the proton, $M_{B}$ is the mass of the recoil nucleus, and $T_{p}$ and $T_{R}$ are kinetic energies of the scattered proton and the recoil nucleus respectively. The missing energy for the reaction is defined by

$$
E_{m i s s}=\omega-T_{p}-T_{R}
$$

while the missing momentum is defined by

$$
\vec{p}_{m i s s}=\vec{p}^{\prime}-\vec{q} .
$$

The reaction plane is defined by $\vec{p}^{\prime}$ and $\vec{p}_{R}$. The angle between the reaction plane and the scattering plane is defined to be the out-of-plane angle, $\phi$. Detection of the proton at $\phi=0^{\circ}$ or $\phi=180^{\circ}$ corresponds to a measurement in the scattering plane, and hence the term "in-plane kinematics". Note that the work presented in this thesis corresponds to in-plane kinematics.

The angle between $\vec{q}$ and $\vec{p}^{\prime}$ is denoted by $\theta_{p q}$. Detection of the proton along $\vec{q}$ such that $\theta_{p q}=0$ corresponds to "parallel kinematics". In this situation, $\vec{p}_{m i s s}$ is parallel to $\vec{q}$. For kinematic settings where $|\vec{q}| \sim\left|\vec{p}^{\prime}\right|$, the detection of the proton on either side of $\vec{q}$ for small values of $\theta_{p q}$ yields $\vec{p}_{m i s s}$ close to being perpendicular to $\vec{q}$, and hence the name "perpendicular kinematics". Fig. 1-4 illustrates these two kinematic conditions. 

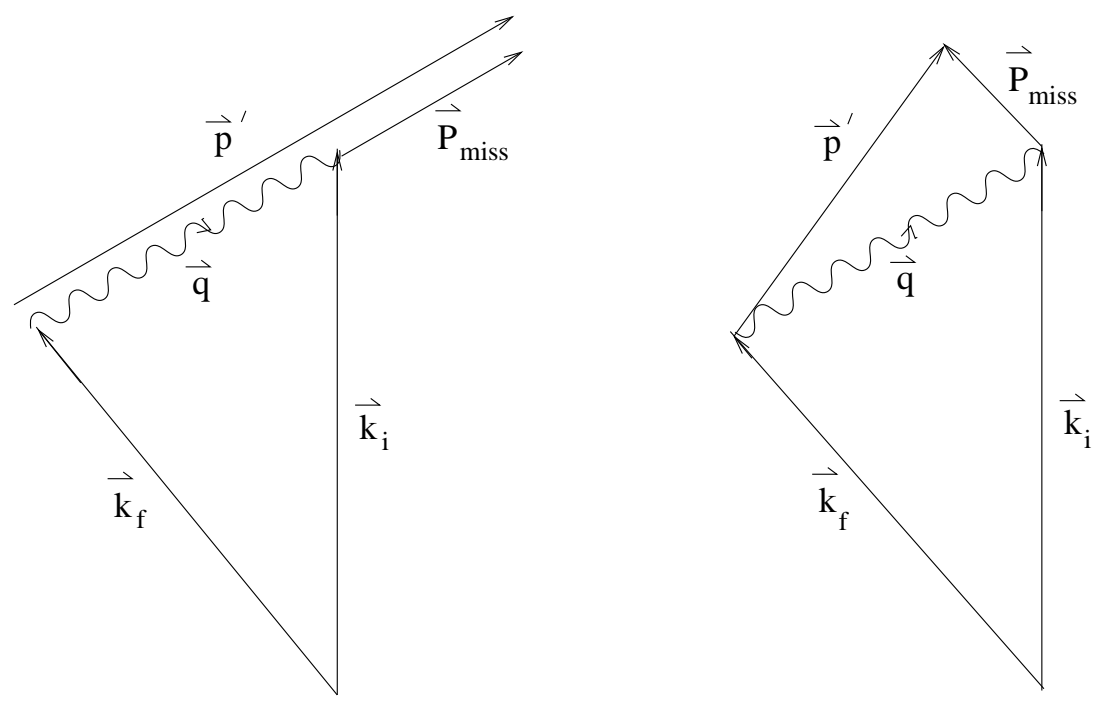

Figure 1-4: A schematic representation of parallel (left) and perpendicular (right) kinematics

\subsubsection{The $\left(e, e^{\prime} p\right)$ reaction formalism}

The (e,e'p) reaction formalism was first developed in references [25], [26], [27], [28], [29], [30].

Only a brief outline of the derivation of the non-polarized (e,e'p) cross section is presented here. For more detail, the reader is referred to a recent review of $\left(e, e^{\prime} p\right)$ physics by Kelly [31]. A thorough review of the formalism is given by Donnelly and Raskin [27]. 


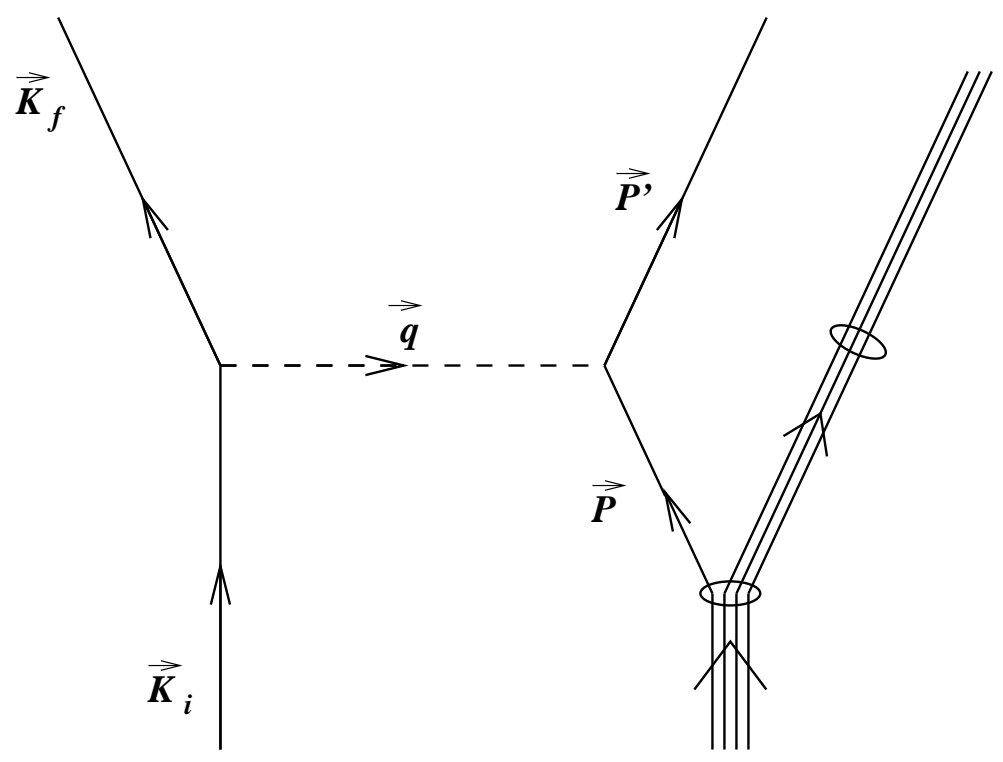

Figure 1-5: The Feynman diagram for the $\mathrm{A}\left(\mathrm{e}, \mathrm{e}^{\prime} \mathrm{p}\right) \mathrm{B}$ reaction in the one photon exchange approximation.

In the first Born approximation (one-photon exchange), the Hamiltonian corresponding to Fig. 1-5 is given by

$$
H=e \int J_{\mu}(x) A^{\mu}(x) d^{4} x
$$

where $J_{\mu}$ is the hadronic electromagnetic current operator $(\rho, \vec{J})$ and $A^{\mu}$ is the Moller potential of the electron.

The Moller potential is determined by solving Maxwell's equations, namely

$$
\square A_{\mu}(x)=-4 \pi j_{\mu}(x),
$$

where $j_{\mu}$ is the electromagnetic current of the electron. For a plane wave electron, with initial and final three momenta $\vec{k}_{i}$ and $\vec{k}_{f}$, the current is given by

$$
j_{\mu}(x)=-i e u_{\vec{k}_{f}} \gamma_{\mu} u_{\overrightarrow{k_{i}}} e^{i q_{\mu} x^{\mu}}
$$

Since the electron polarizations are not measured in this experiment, the spin indices have been suppressed in 1.13 for the sake of simplicity.

The (e,e'p) cross section is proportional to the matrix element $M^{2}$ for diagram 1-5. 
Given the Hamiltonian 1.11, the square of the matrix element can be expressed as the scalar contraction of $\eta_{\mu \nu}$ and $W_{\mu \nu}$, the Lorentz tensors that represent the leptonic and hadronic current contributions [25]

$$
\left|M^{2}\right|=\eta_{\mu \nu} W_{\mu \nu}
$$

For a plane wave electron the leptonic tensor is given by

$$
\eta_{\mu \nu}=K_{\mu} K_{\nu}-q_{\mu} q_{\nu}-Q^{2} g_{\mu \nu}
$$

where $K=k_{i}+k_{f}$.

The corresponding hadronic tensor is given by

$$
W_{\mu \nu}=\left\langle J_{\mu} J_{\nu}^{\dagger}\right\rangle
$$

where the angular brackets denote an average over initial states and a summation over final states.

For unpolarized particles, the hadronic tensor is symmetric and can be constructed from the three independent four vectors, namely the four momentum transfer $\left(q^{\mu}\right)$, the target nucleus four momentum $\left(P_{A}^{\mu}\right)$ and the ejectile nucleon four momentum $\left(p^{\prime \mu}\right)$.

Using the constraints imposed by the conservation of the hadronic current

$$
q_{\mu} W_{\mu \nu}=W_{\mu \nu} q_{\nu}=0
$$

the contraction of leptonic and hadronic tensors can be written in the simple form

$$
\eta_{\mu \nu} W_{\mu \nu}=\left\langle K \cdot J K \cdot J^{\dagger}-Q^{2} J \cdot J^{\dagger}\right\rangle
$$

This is further simplified by the elimination of the longitudinal component of the 
hadronic current in favor of the charge using the continuity equation ${ }^{1}$,

$$
J_{z}=\frac{\omega}{|q|} \rho
$$

After some algebra, the six-fold differential cross section for $\left(e, e^{\prime} p\right)$ may be obtained

$$
\frac{d^{6} \sigma}{d \Omega_{e} d \Omega_{p} d \omega d E_{p^{\prime}}}=K \sigma_{M}\left(V_{\mathrm{L}} R_{\mathrm{L}}+V_{\mathrm{T}} R_{\mathrm{T}}+V_{\mathrm{LT}} R_{\mathrm{LT}} \cos \phi+V_{\mathrm{TT}} R_{\mathrm{TT}} \cos 2 \phi\right)
$$

where

$$
\begin{aligned}
K & =\frac{p^{\prime} E_{p^{\prime}}}{(2 \pi)^{3}}, \\
\sigma_{M} & =\frac{\alpha^{2} \cos ^{2}\left(\theta_{e} / 2\right)}{4 E_{i}^{2} \sin ^{4}\left(\theta_{e} / 2\right)}, \\
V_{\mathrm{L}} & =\frac{Q^{4}}{\vec{q}^{4}}, \\
V_{\mathrm{T}} & =\frac{Q^{2}}{2 \vec{q}^{2}}+\tan ^{2}\left(\theta_{e} / 2\right), \\
V_{\mathrm{LT}} & =\frac{Q^{2}}{\vec{q}^{2}}\left[\frac{Q^{2}}{\vec{q}^{2}}+\tan ^{2}\left(\theta_{e} / 2\right)\right]^{\frac{1}{2}}, \\
V_{\mathrm{TT}} & =\frac{Q^{2}}{2 \vec{q}^{2}}
\end{aligned}
$$

The four independent response functions $R_{\mathrm{L}}, R_{\mathrm{T}}, R_{\mathrm{LT}}$, and $R_{\mathrm{TT}}$ contain all the information that can be extracted from the hadronic system using the (e,e'p) reaction. They can be expressed in terms of the components of the hadronic current and the charge density in the following manner:

$$
\begin{aligned}
R_{\mathrm{L}} & =<\rho \rho^{+}>, \\
R_{\mathrm{T}} & =<J_{\|} J_{\|}^{+}+J_{\perp} J_{\perp}^{+}>, \\
R_{\mathrm{LT}} \cos \phi & =-<\rho J_{\|}^{+}+J_{\|} \rho^{+}>, \\
R_{\mathrm{TT}} \cos 2 \phi & =<J_{\|} J_{\|}^{+}-J_{\perp} J_{\perp}^{+}>,
\end{aligned}
$$

\footnotetext{
${ }^{1}$ The replacement of the the longitudinal component of the hadronic current by the charge was originally suggested by de Forest [32], [33]. This prescription is associated with the Coulomb gauge.
} 
where $J_{\|}$and $J_{\perp}$ are the components of the hadronic current orthogonal to $\vec{q}$ such that $J_{\|}$lies in the scattering plane and $J_{\perp}$ is perpendicular to that plane.

The longitudinal response $R_{\mathrm{L}}$ is related to the hadronic charge density. The transverse response $R_{\mathrm{T}}$ is due to the incoherent sum of the contributions from the two components of the current perpendicular to $\vec{q}$. $R_{\mathrm{LT}}$ and $R_{\mathrm{TT}}$ arise from the interference of the different components of the nuclear current.

\subsection{Plane Wave Impulse Approximation}

Fig. 1-5 corresponds to the Plane Wave Impulse Approximation (PWIA). The basic assumptions of PWIA are:

- The total energy and momentum of a single virtual photon is absorbed by a single nucleon.

- The struck nucleon leaves the nucleus without interacting with the rest of the nucleus, and the outgoing nucleon may be represented by a plane wave.

- The knocked-out nucleon, not a spectator nucleon, is the one detected in the experiment.

- The nucleus can be described by an independent particle model.

In PWIA, the conservation of linear momentum requires that the initial momentum of the struck nucleon $\left(\vec{p}_{i}\right)$ is equal to the missing momentum

$$
\vec{p}_{i}=\vec{p}^{\prime}-\vec{q}=\vec{p}_{m i s s}
$$

Frullani and Mougey [34] have applied these assumptions to allow for factorization of the $\left(\mathrm{e}, \mathrm{e}^{\prime} \mathrm{p}\right)$ cross section

$$
\frac{d^{6} \sigma}{d \Omega_{e} d \Omega_{p} d \omega d E_{p^{\prime}}}=K \sigma_{e p} S\left(E_{m i s s}, \vec{p}_{m i s s}\right)
$$

where $K$ is a kinematic factor and $\sigma_{e p}$ is the off-shell electron-proton cross section for a bound proton. The spectral function $S\left(E_{m i s s}, \vec{p}_{m i s s}\right)$ is the probability of knocking out 
a nucleon and leaving the residual nucleus with momentum $\vec{p}_{R}=-\vec{p}_{i}$ and excitation energy $E_{\text {miss }}$.

\subsection{Distorted Wave Impulse Approximation}

Of the assumptions made within the PWIA framework, the second is the least likely to be valid, since the ejected nucleon interacts with the residual nucleus via the strong interaction. The Distorted Wave Impulse Approximation (DWIA) takes these Final State Interactions (FSI) into account, while at the same time maintaining the other three assumptions. Fig. 1-6 presents the diagram for DWIA.

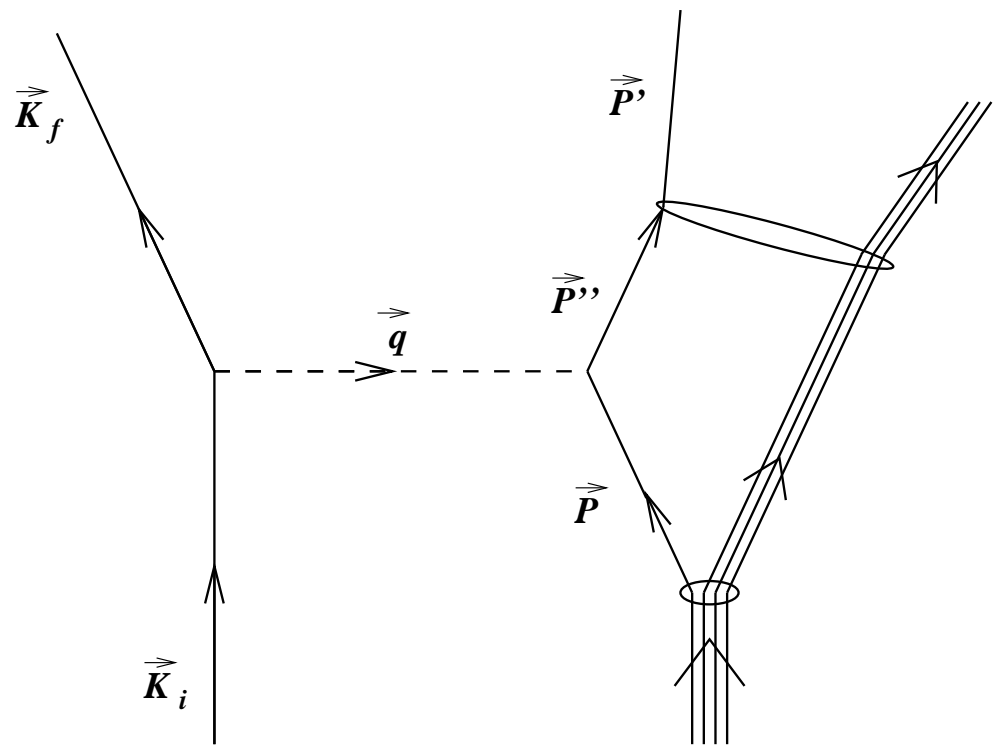

Figure 1-6: A(e,e'p)B reaction in the Distorted Wave Impulse Approximation.

In DWIA, the FSI are usually addressed using an optical potential to derive the distorted wave for the ejected nucleon. The optical potentials are obtained from fits to elastic nucleon scattering data. A description of the optical potential formalism and a detailed comparison of several widely used optical potentials may be found in [31]. 


\subsection{Previous $\left(\mathbf{e}, \mathbf{e}^{\prime} \mathbf{p}\right)$ measurements in the $E_{m i s s}$ continuum}

A series of ${ }^{12} \mathrm{C}\left(\mathrm{e}, \mathrm{e}^{\prime} \mathrm{p}\right)$ experiments performed at MIT-Bates which sampled various kinematic regions have revealed much higher strength in the $E_{\text {miss }}$ continuum than was expected for single particle knockout. All the measurements summarized below (except the measurement by Holtrop et al.) were made in parallel kinematics.

Ulmer et al. [35], [36] separated the longitudinal and transverse response functions for the ${ }^{12} \mathrm{C}\left(\mathrm{e}, \mathrm{e}^{\prime} \mathrm{p}\right)$ reaction in the quasielastic region at $q=400 \mathrm{MeV} / \mathrm{c}$ and $\omega=120 \mathrm{MeV}$. These separated response functions and the difference between the corresponding longitudinal and transverse spectral functions is shown in Fig. 1-7.

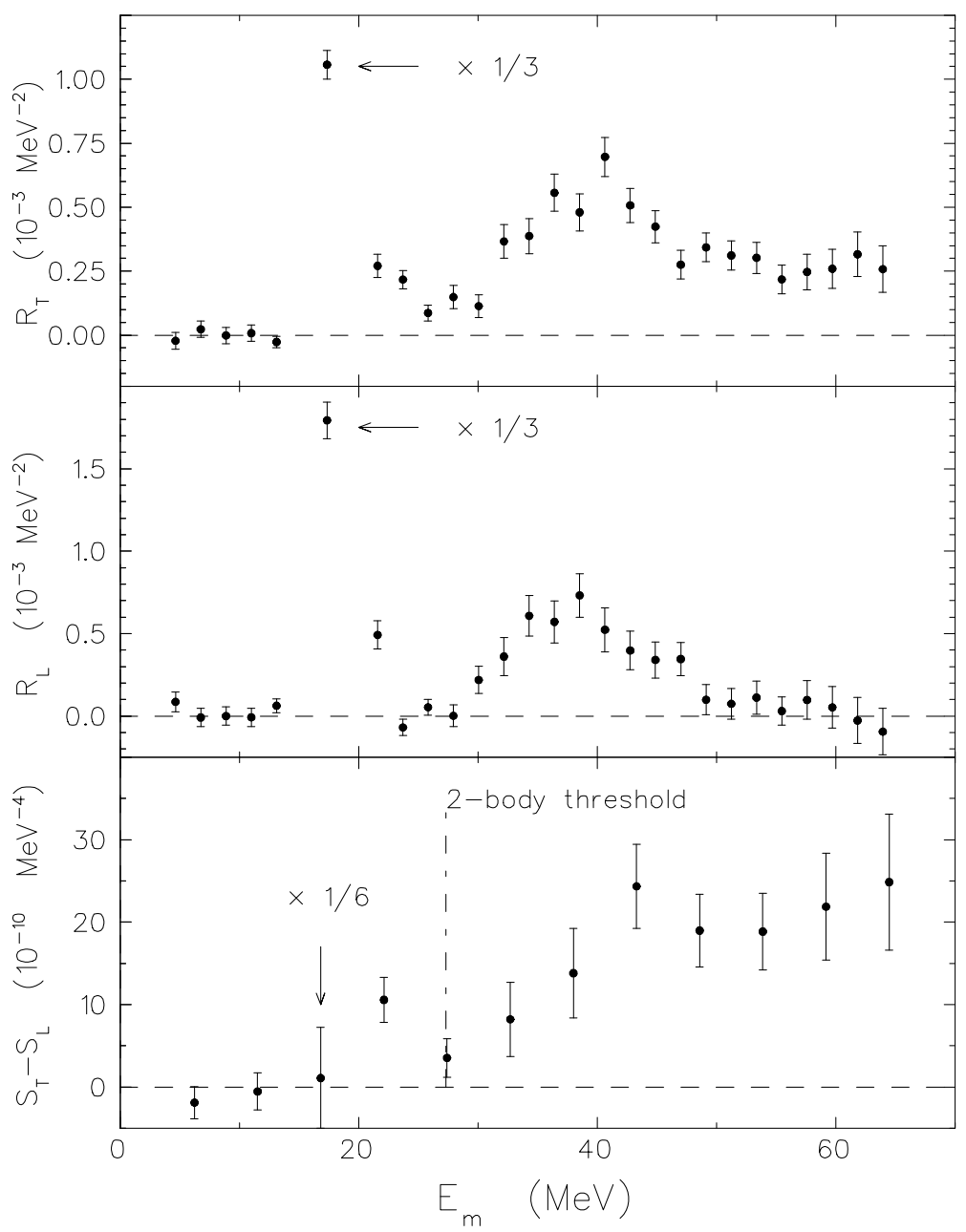

Figure 1-7: Longitudinal and transverse responses for the ${ }^{12} \mathrm{C}\left(\mathrm{e}, \mathrm{e}^{\prime} \mathrm{p}\right)$ reaction in the quasi-elastic region at $q=400 \mathrm{MeV} / \mathrm{c}$ and $\omega=120 \mathrm{MeV}$. The bottom plot shows the the difference between the corresponding longitudinal and transverse spectral functions. 
In the figure, $1 \mathrm{p}_{3 / 2}$ strength can be seen as a sharp peak around $17 \mathrm{MeV}$, while the broad peak centered around $40 \mathrm{MeV}$ can be identified as $1 \mathrm{~s}_{1 / 2} . R_{L}$ and $R_{T}$ spectra behave similarly for $E_{\text {miss }}<50 \mathrm{MeV}$. However, after $50 \mathrm{MeV}$, excess transverse strength persists up to the highest missing energy measured while the longitudinal strength appears to go to zero. For the $1 \mathrm{p}_{3 / 2}$ valence state region, the difference between the longitudinal and transverse spectral functions is close to zero, as expected for a free proton. This difference starts to grow above the two particle emission threshold $\left(E_{\text {miss }}=28 \mathrm{MeV}\right)$ and appears to reach a plateau for $E_{m i s s}>50 \mathrm{MeV}$. The excess transverse strength indicates an emerging non single-particle reaction channel after the two-particle emission threshold.

Fig. 1-8 shows the ${ }^{12} \mathrm{C}\left(\mathrm{e}, \mathrm{e}^{\prime} \mathrm{p}\right)$ cross section measured by Lourie et al. [37] [38] in the dip region at $q=400 \mathrm{MeV} / \mathrm{c}$ and $\omega=200 \mathrm{MeV}$ up to $E_{\text {miss }}=150 \mathrm{MeV}$. In this case, the $1 \mathrm{~s}_{1 / 2}$ tail appears extend to about $E_{\text {miss }}=65 \mathrm{MeV}$, after which an almost flat continuum strength extends to the deepest measured missing energy. The dashed line underneath the S-shell bump is an extrapolation of the flat continuum strength. It is possible that the strength above this line is due to single particle knockout from $1 \mathrm{~s}_{1 / 2}$. However, the Ulmer data previously discussed (Fig. 1-7) shows that the longitudinal response is consistent with zero beyond $E_{\text {miss }}=50 \mathrm{MeV}$. This indicates the strength in the " $1 \mathrm{~s}_{1 / 2}$ tail" above $E_{\text {miss }}=50 \mathrm{MeV}$ might be due to other sources. 


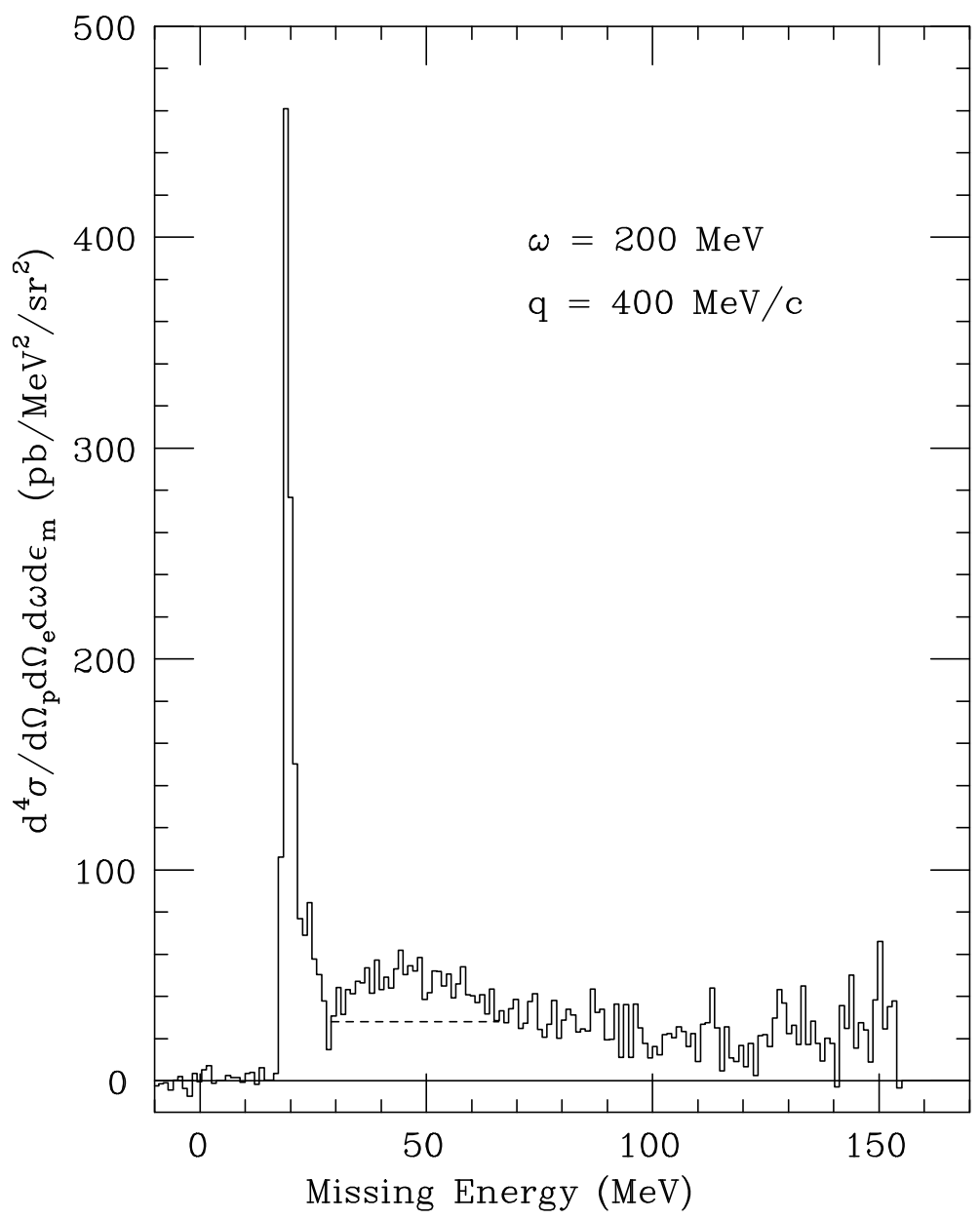

Figure 1-8: Radiatively corrected missing energy spectrum for ${ }^{12} \mathrm{C}\left(\mathrm{e}, \mathrm{e}^{\prime} \mathrm{p}\right)$ in the dip region with $q=400 \mathrm{MeV} / \mathrm{c}$ and $\omega=200 \mathrm{MeV}$ [37]. The dashed line underneath the S-shell bump is an extrapolation of the flat continuum strength.

Weinstein et al. [39] [40] measured the ${ }^{12} \mathrm{C}\left(\mathrm{e}, \mathrm{e}^{\prime} \mathrm{p}\right)$ cross section as a function of missing energy in quasielastic kinematics at momentum transfers of 585, 775 and 827 $\mathrm{MeV} / \mathrm{c}$ (Fig. 1-9). These spectra exhibit the same qualitative features as those of Lourie et al., however, the relative strength in the continuum compared to the $1 \mathrm{~s}_{1 / 2}$ strength is much lower for the quasielastic region than for the dip region. 


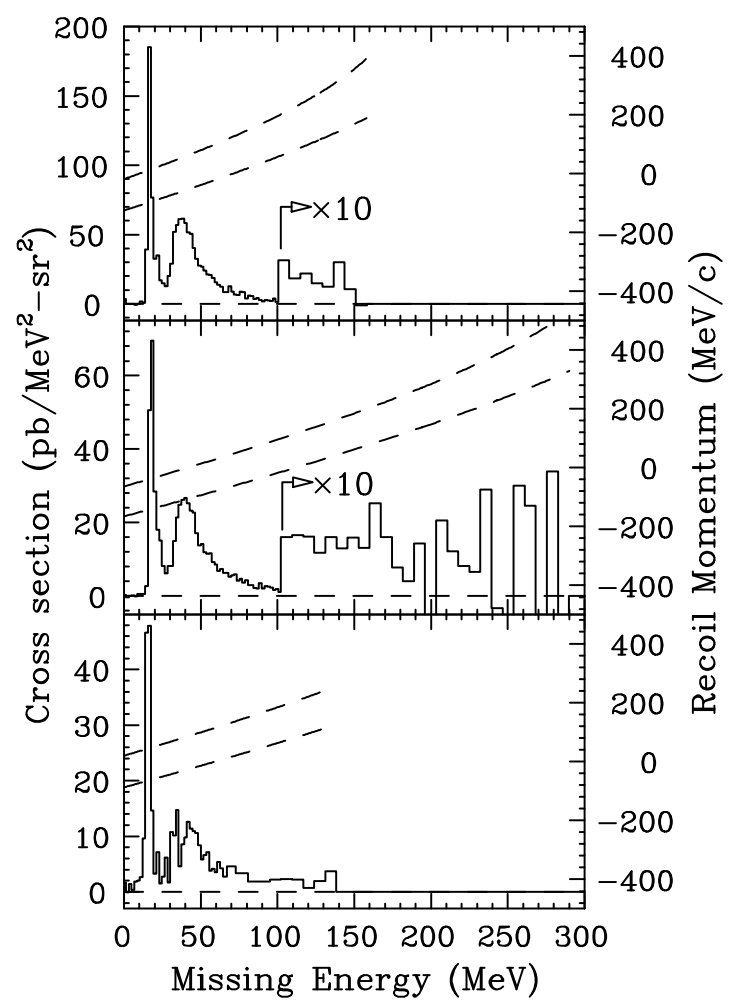

Figure 1-9: Radiatively corrected missing energy spectra for ${ }^{12} \mathrm{C}\left(\mathrm{e}, \mathrm{e}^{\prime} \mathrm{p}\right)$ obtained in parallel kinematics near the quasielastic peak [39].

Fig. 1-10 shows the ${ }^{12} \mathrm{C}\left(\mathrm{e}, \mathrm{e}^{\prime} \mathrm{p}\right)$ cross section measured by Morrison et al. [41] in the quasielastic region. For the $(|\vec{q}|=970 \mathrm{MeV}, \omega=300 \mathrm{MeV})$ setting on the lower $\omega$ side of the quasielastic peak, the continuum strength above $60 \mathrm{MeV}$ appears to be small. However, the measurement at the top of the quasielastic peak $(|\vec{q}|=990 \mathrm{MeV}$, $\omega=475 \mathrm{MeV}$ ) shows a flat continuum spectrum similar to the results of Lourie and Weinstein. There appears to be no increase in the cross section at pion threshold, $E_{\text {miss }} \approx 155 \mathrm{MeV}$. Presumably pion production is not important in the quasielastic region. 


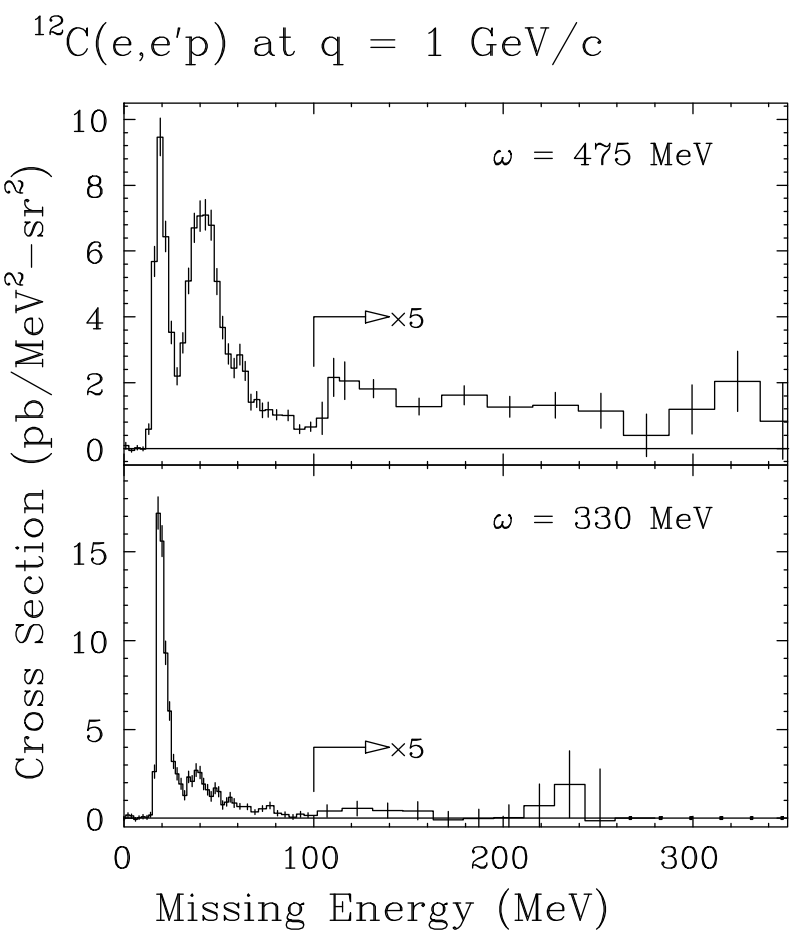

Figure 1-10: Radiatively corrected missing energy spectra for ${ }^{12} \mathrm{C}\left(\mathrm{e}, \mathrm{e}^{\prime} \mathrm{p}\right)$ obtained in parallel kinematics near the quasielastic peak at high momentum transfer [41]. $100 \mathrm{MeV}<E_{\text {miss }}$ data have been scaled by a factor of 5 .

Holtrop et al measured quasielastic ${ }^{12} \mathrm{C}\left(\mathrm{e}, \mathrm{e}^{\prime} \mathrm{p}\right)$ cross sections in perpendicular kinematics at $(|\vec{q}|=404 \mathrm{MeV}, \omega=110 \mathrm{MeV})$ and used them to extract the interference response function $R_{L T}$ for $10 \mathrm{MeV}<E_{\text {miss }}<60 \mathrm{MeV}$. As Fig. 1-11 shows, this measurement has demonstrated that $R_{L T}$ is consistent with zero for $E_{\text {miss }}>50 \mathrm{MeV}$. This observation is in agreement with the Ulmer results which showed that $R_{L}$ is consistent with zero for $E_{\text {miss }}>50 \mathrm{MeV}$.

These experiments have demonstrated the presence of substantial cross section in the $E_{\text {miss }}$ continuum above $50 \mathrm{MeV}$. It is speculated that this indicates a new reaction channel appearing at high $E_{\text {miss }}$. The evidence that the continuum cross section is predominantly transverse starting around the two-particle emission threshold indicates that the emerging reaction channel may be two-body or multi-body knockout. 


\section{${ }^{12} \mathrm{C}(\mathrm{e}, \mathrm{e} \cdot \mathrm{p})$ Cross Sections}
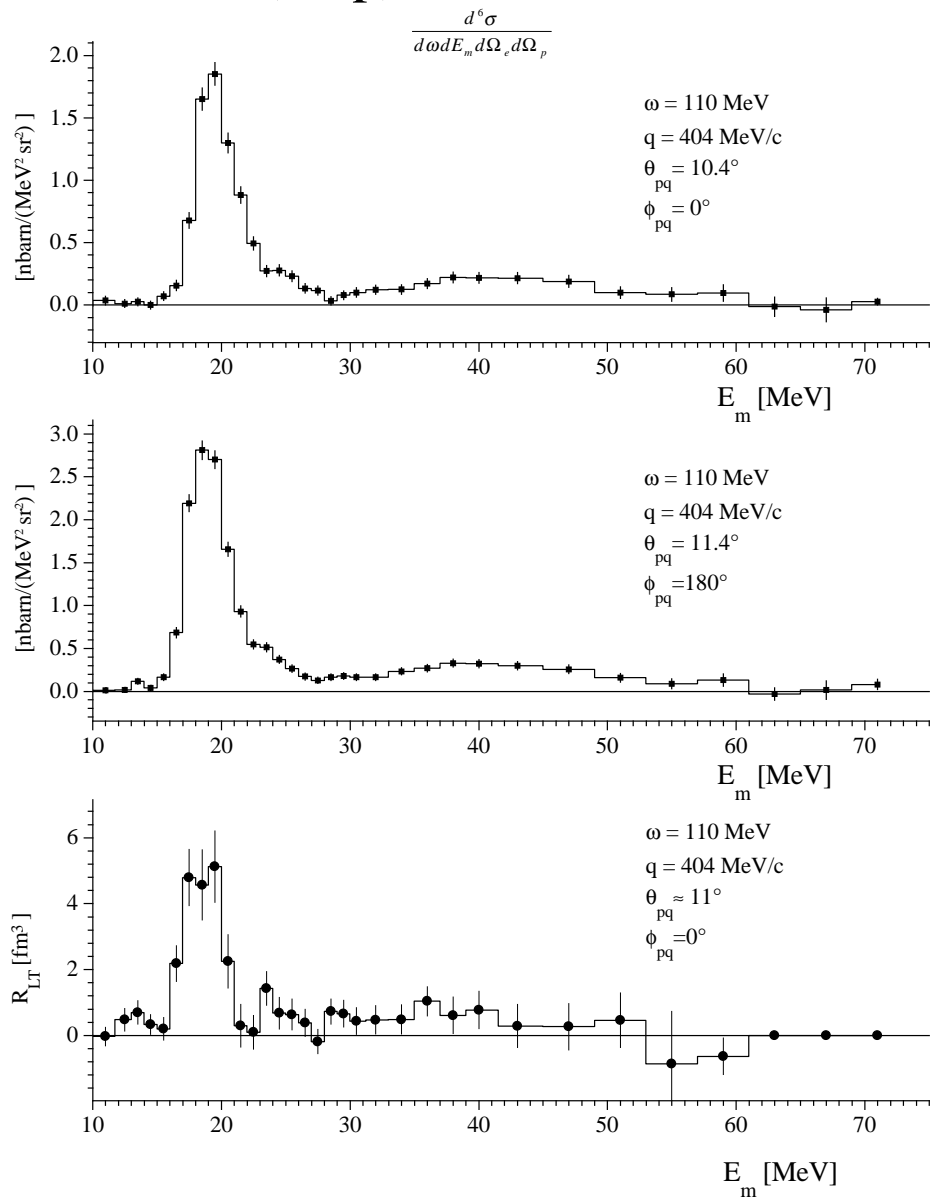

Figure 1-11: The cross sections and $R_{L T}$ for the quasielastic ${ }^{12} \mathrm{C}\left(\mathrm{e}, \mathrm{e}^{\prime} \mathrm{p}\right)$ reaction measured by Holtrop et al. $[42]$ at $(|\vec{q}|=404 \mathrm{MeV}, \omega=110 \mathrm{MeV})$.

There have been several theoretical attempts to explain the continuum strength using many-body knockout models. Recently, Ryckebusch [43] has calculated the (e,e'pn) and (e,e'pp) contributions to the (e,e'p) cross section in the continuum. This calculation includes two-nucleon knockout mediated by pion-exchange currents, intermediate $\Delta$ creation, and central short-range correlations. Fig. 1-12 shows a comparison of the Ryckebusch calculation to the response functions separated by Ulmer. The solid curves shown in the plots are single-particle knockout strengths for $1 \mathrm{~s}_{1 / 2}$ calculated by Ryckebusch et al. These calculations were performed in a self-consistent Hartree-Fock (HF) Random Phase Approximation (RPA) framework [44], [45], [46]. To account for the lack of an absorptive imaginary potential in the treatment of FSI 
in the HF-RPA approach, a spectroscopic factor has to be used to normalize the calculation. A factor of 0.5 has been adopted in the present case by fitting the $R_{L}$ distribution measured by Ulmer et al.

As can be seen in the figure, the shape of $R_{L}$ is correctly predicted by the calculation. However, the single-particle knockout contribution to $R_{T}$ (normalized with the spectroscopic factor determined from $R_{L}$ ) underestimates the data, and the discrepancy increases with increasing $E_{\text {miss }}$. The two-body knockout contribution is not sufficient to explain this discrepancy.

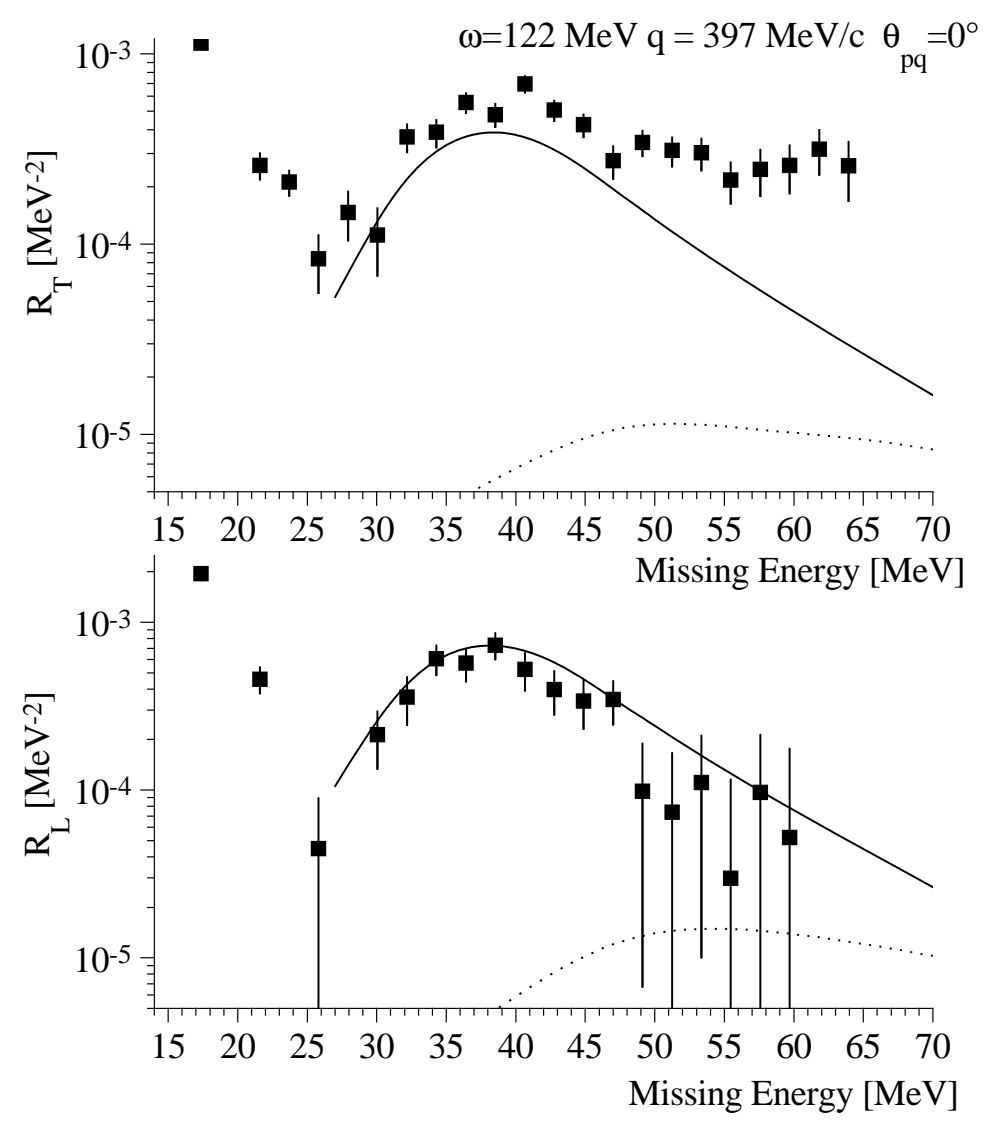

Figure 1-12: Comparison of separated $R_{L}$ and $R_{T}$ response functions from Ulmer et al. [35], [36] with an HF-RPA calculation by Ryckebusch [43]. The solid lines are the predicted contributions from the $1 \mathrm{~s}_{1 / 2}$ single particle knockout, while the dashed lines are the contributions due to $\left(e, e^{\prime} \mathrm{pn}\right)$ and (e,e'pp) knockout. Note that a spectroscopic factor of 0.5 has been adopted. 
It has also been suggested that short-range and tensor correlations could play a major role in populating the high $E_{\text {miss }}$ continuum. Muther et al. [47] calculated the effects of these correlations in ${ }^{16} \mathrm{O}$ and have shown that they cause a depletion of strength in conventional quasi-hole states to the benefit of high- $E_{m i s s}$, high- $P_{\text {miss }}$ channels. In an ${ }^{16} \mathrm{O}\left(\mathrm{e}, \mathrm{e}^{\prime} \mathrm{p}\right)$ experiment performed at Mainz, Blomqvist et al. [48] have measured the ${ }^{16} \mathrm{O}\left(\mathrm{e}, \mathrm{e}^{\prime} \mathrm{p}\right)$ cross section for $0 \mathrm{MeV}<E_{\text {miss }}<120 \mathrm{MeV}$ at several values of $P_{\text {miss }}$ ranging from $120 \mathrm{MeV} / \mathrm{c}$ to $590 \mathrm{MeV} / \mathrm{c}$. The results from this experiment (Fig. 1-13) have shown that the relative strength at high $E_{\text {miss }}$ compared to the strength in the valence states increases with increasing $P_{\text {miss }}$, in qualitative agreement with the prediction by Muther et al. However, it must be noted that while the lower $P_{\text {miss }}$ cross sections reported by Blomqvist et al. have been measured in parallel kinematics, the higher $P_{\text {miss }}$ settings have been obtained in perpendicular kinematics, which makes it difficult to make a systematic comparison between the cross sections measured at different $P_{\text {miss }}$. The kinematic limitations imposed by the relatively low beam energy available at Mainz prevented measuring the different $P_{\text {miss }}$ cross sections under similar kinematic conditions. The large strength observed at Mainz in the high $\left(P_{\text {miss }}, E_{\text {miss }}\right)$ region is possibly of non single-particle origin. The separation of longitudinal and transverse components of this cross section should greatly assist in the interpretation of the origin of this high strength. 


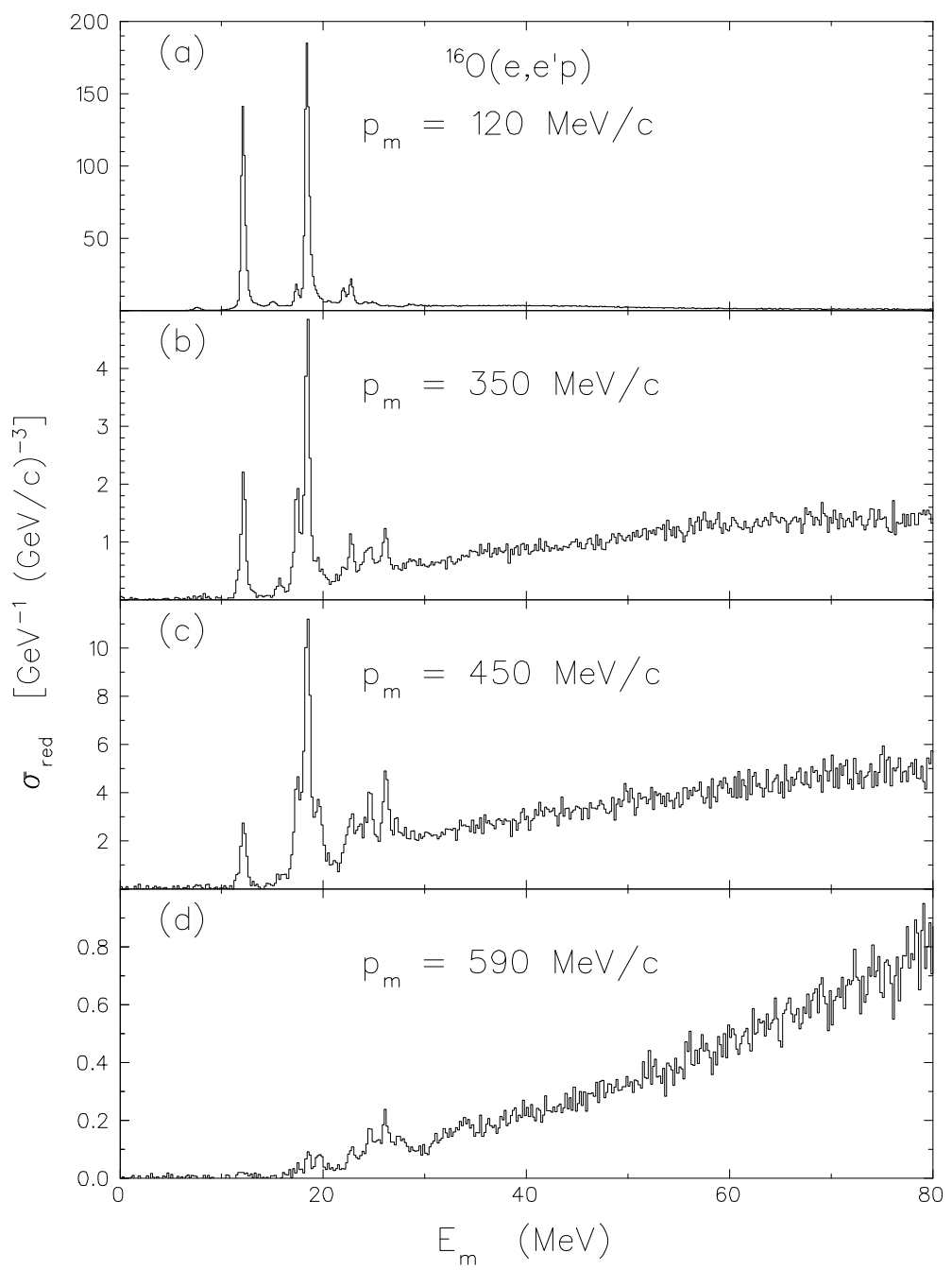

Figure 1-13: Missing energy distributions measured at Mainz by Blomqvist for ${ }^{16} \mathrm{O}\left(\mathrm{e}, \mathrm{e}^{\prime} \mathrm{p}\right)$ are presented as reduced cross sections without radiative unfolding. The figure is from reference [31].

It should be noted that there has not been a single response function separation on a complex nucleus in the large $\left(P_{\text {miss }}, E_{\text {miss }}\right)$ region ever performed at any laboratory ${ }^{2}$.

\subsection{The present experiment}

With its continuous electron beam of relatively high energy, Jefferson Laboratory overcomes many of the limitations imposed on $\left(e, e^{\prime} p\right)$ experiments at other laboratories. Some of the capabilities available at the Jefferson lab include

\footnotetext{
${ }^{2}$ As described previously, Ulmer et al. and Holtrop et al. have performed response function separations for ${ }^{12} \mathrm{C}\left(\mathrm{e}, \mathrm{e}^{\prime} \mathrm{p}\right)$ up to $E_{m i s s}=65 \mathrm{MeV}$. However these experiments were performed at relatively low missing momenta.
} 
- high trues-to-accidental ratio due to the continuous electron beam. The truesto-accidental ratio at Jefferson Lab is about two orders of magnitude higher than at a conventional pulsed beam machine. This enables a better separation of cross sections measured in the high $\left(P_{\text {miss }}, E_{\text {miss }}\right)$ region, as the yield due to accidental coincidences is greatly suppressed.

- relatively high beam energy $(\sim 4 \mathrm{GeV})$ that allows the exploration of large ranges of missing energies and missing momenta under similar kinematic conditions.

Equipped with these new capabilities, this experiment was conceived to answer some questions raised by the previous experiments:

- Is the excess strength and the response function behavior seen previously in the ${ }^{12} \mathrm{C}\left(\mathrm{e}, \mathrm{e}^{\prime} \mathrm{p}\right)$ high $E_{\text {miss }}$ continuum also present at even higher momentum transfer in ${ }^{16} \mathrm{O}$ ? Is the observed high $E_{\text {miss }}$ behavior independent of the nucleus and the momentum transfer?

- Is the Mainz observation of high strength at large $\left(P_{m i s s}, E_{m i s s}\right)$ still observed when the missing momentum is varied under similar kinematic conditions; perpendicular kinematics in the quasielastic region?

- What is the composition of the high $\left(P_{m i s s}, E_{m i s s}\right)$ cross section in terms of the separated responses?

- How does the cross section measured in the dip region compare to the cross section measured in the quasielastic region? Does the importance of $\Delta$ degrees of freedom increase in going from the quasielastic region to the dip region? 


\section{Chapter 2}

\section{The Experiment}

This work represents the first physics experiment performed in Hall A at the Thomas Jefferson National Accelerator Facility. Subsequent to a year of commissioning activities, the experiment was performed during the summer of 1997. The Hall A High Resolution Spectrometer (HRS) pair was utilized in so-called "standard" configuration. The first part of this chapter describes the CEBAF accelerator at the Jefferson Lab and the Hall A experimental setup, while the latter part presents the kinematics and the procedure of the experiment.

\section{$2.1 \quad$ Jefferson Lab}

The Thomas Jefferson National Accelerator Facility, the world's premier medium energy electron scattering laboratory, is located in Newport News, Virginia. The facility consists of a state-of-the art continuous wave electron accelerator, three complementary experiment halls that utilize the beam to explore different aspects of nuclear physics, a free electron laser facility and an applied research center.

The CEBAF accelerator at Jefferson Lab is capable of delivering high quality continuous electron beams of up to $4 \mathrm{GeV}$ as of 1997. Future plans include upgrades to $8 \mathrm{GeV}, 10 \mathrm{GeV}$ and eventually to $20 \mathrm{GeV}$. The accelerator site layout is shown in Fig. 2-1. 


\section{MACHINE CoNFIGURATION UUefrerson tam}

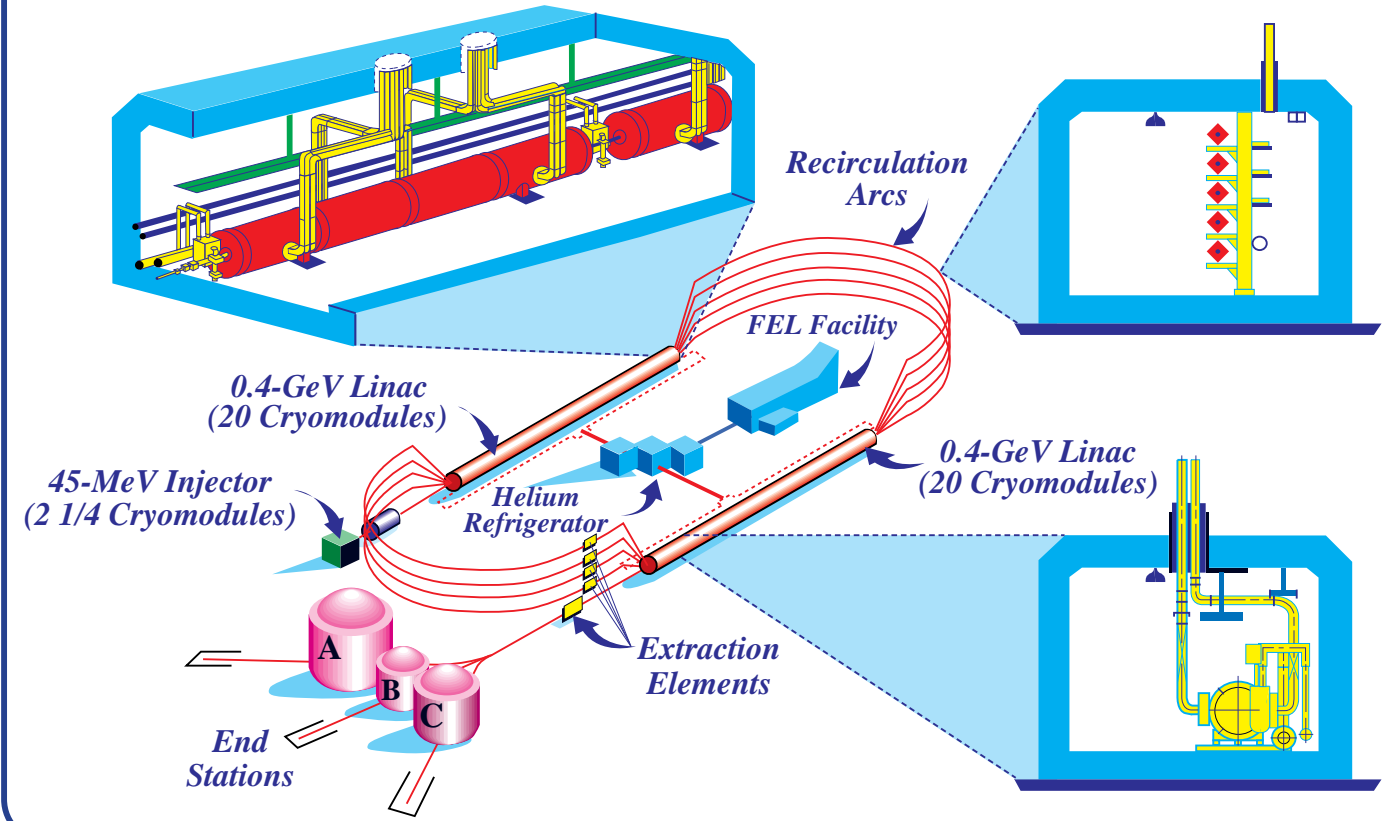

Figure 2-1: Layout of the Jefferson Lab accelerator site.

The electron beam used in the experiment is generated in the following manner. The injector delivers $45 \mathrm{MeV}$ electrons to the race-track type accelerator, which consists of two $0.4 \mathrm{GeV}$ linacs. With each pass through the machine an electron gains $0.8 \mathrm{GeV}$. At the beam switch-yard, labeled "extraction elements" in the figure, the beam is extracted and delivered to three experimental halls simultaneously.

The machine is operated in electron-linac mode, with the electron bunches "riding the crest" of the sinusoidal RF wave without longitudinal focusing, relying on the ultra-relativistic motion of the electrons. At an injection energy of $45 \mathrm{MeV}$, the total accumulated phase lag (with respect to $\beta=1$ particles) is less than $2^{\circ}$ over five passes through the machine.

The linacs use CEBAF-Cornell type superconducting cavities. The five-cell, 1497 $\mathrm{MHz}$ elliptical cavity provides acceleration gradients in excess of $5 \mathrm{MeV} / \mathrm{m}$. Each cavity is enclosed in a $2.0 \mathrm{~K}$ liquid helium bath within a cryounit. Eight cryounits are combined into a single cryomodule. Cryomodules are connected to their neighbors 
by a warm (non-superconducting) section which consists of the beam pipe, beam monitors, and magnets to focus and steer the beam. Each of the two linacs contains 20 cryomodules with each cryomodule providing an acceleration gradient of $20 \mathrm{MeV}$.

\section{$2.2 \quad$ Hall A}

Fig. 2-2 shows the layout of Experiment Hall A. The hall is circular in shape, and has a diameter of $53 \mathrm{~m}$. The bulk of the volume of the hall is underground, well shielded with concrete and a thick layer of earth. Beam enters the hall from the lower left hand corner of the figure. The beamline is instrumented with beam position and beam current monitors (see section 2.2.1). The beam is incident upon the scattering chamber, in essence a large vacuum can, located at the center of the hall. For this experiment, the scattering chamber contained a waterfall target (see section 2.2.2).

The hall is instrumented with the HRS pair shown in the figure in their standard configuration. While the spectrometers are labeled "Electron Spectrometer" and "Hadron spectrometer" in the figure, it should be noted that either spectrometer can be configured for the detection of either type of particle by simply switching the field polarity of the magnets.

The bulk of the electrons incident upon the scattering chamber and the waterfall target do not interact and are transported to a well shielded, isolated beam dump (not shown in the figure). Some of the electrons that scatter may be detected in the electron spectrometer while the protons which are knocked-out from the target nuclei may be detected in the hadron spectrometer. The detection of an electron and a proton within a small time window set by the experimenter is a signature of a coincidence (e,e'p) event. 


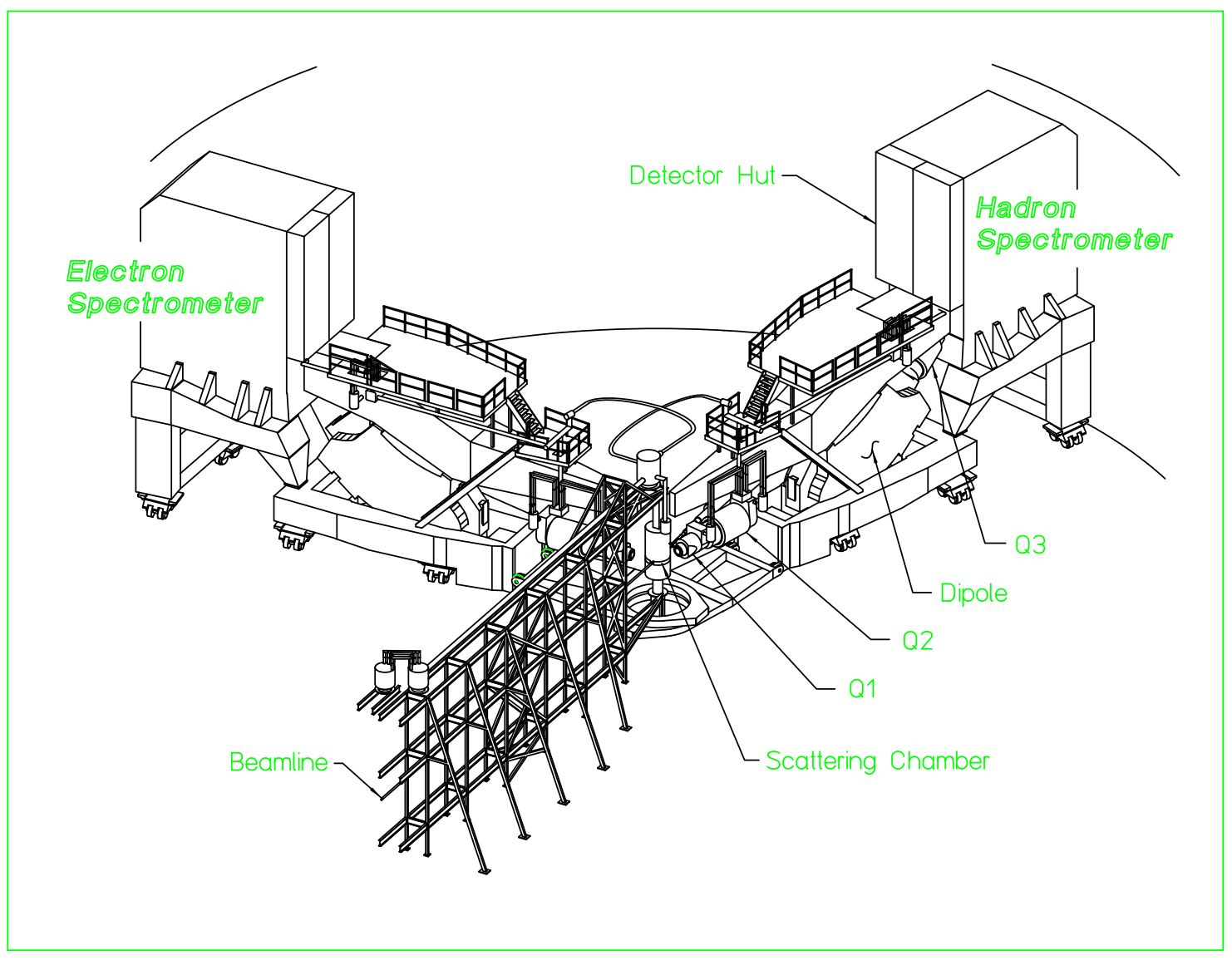

Figure 2-2: Hall A

\subsubsection{Beam line equipment}

\subsubsection{Beam current monitors}

A detailed discussion of the Hall A beam charge monitoring system is given in [49], and only a brief overview is presented here. The beam current delivered to the hall is measured by two RF Beam Cavity Monitors (BCM) placed $24.5 \mathrm{~m}$ upstream of the target. When the cavities are tuned to the frequency of the beam $(1497 \mathrm{MHz})$, they output voltage levels proportional to the beam current. Sandwiched between the two Beam Current Monitors is an Unser monitor [50], [51] used to calibrate the cavities. The Unser is a direct current transformer consisting of two identical toroidal cores driven in opposite ways by an external source. The Unser is calibrated by sending in a current generated by a high-precision source, through a wire placed within the cores to simulate the beam. 


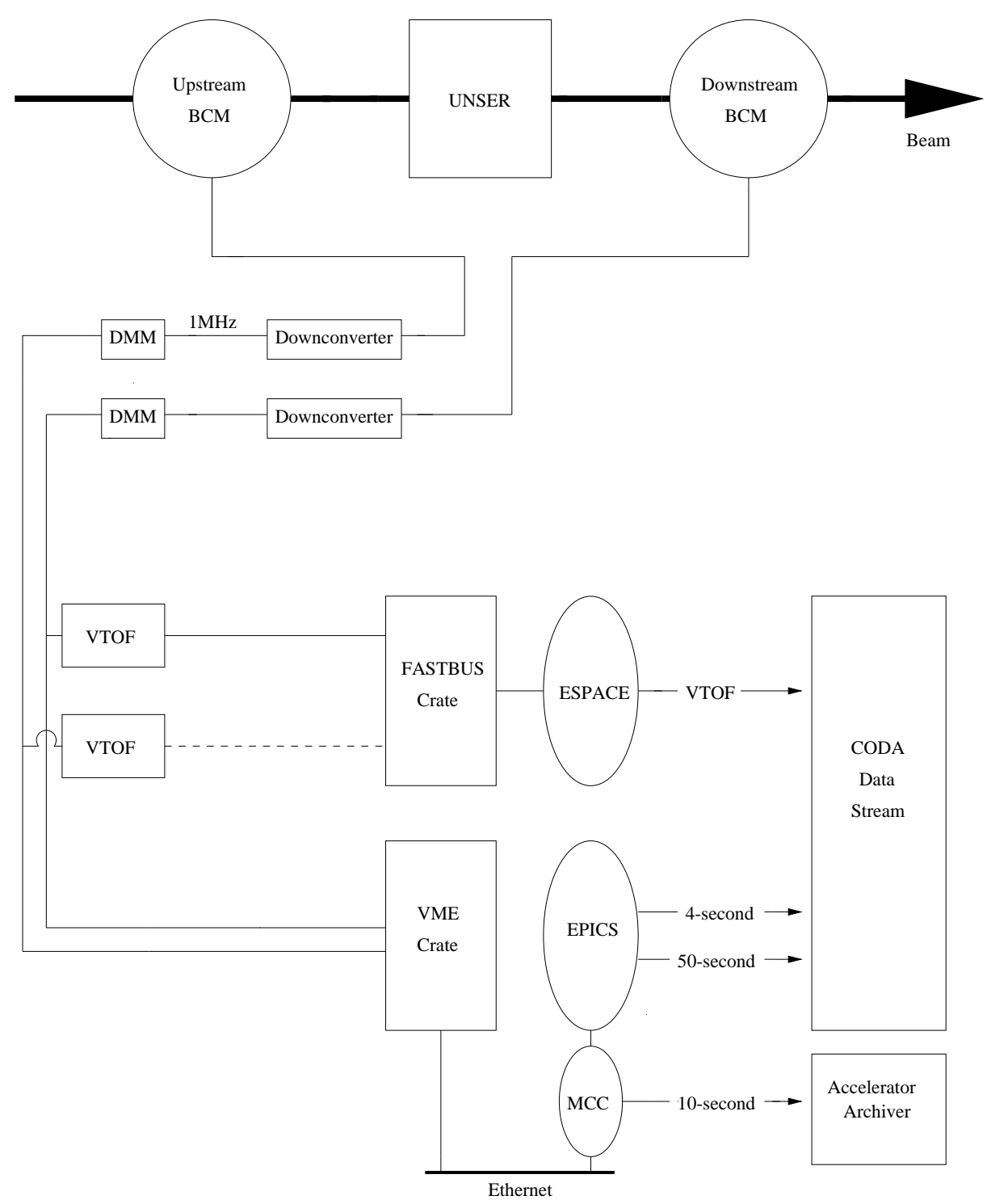

Figure 2-3: A block diagram showing the schematics of Hall A beam current monitoring at the time of this experiment. The acronyms used in the figure are defined in table 2.1. Figure courtesy of Brian Diederich.

\begin{tabular}{|cl|}
\hline BCM & Beam Cavity Monitor \\
DMM & Digital Multi-Meter \\
V-to-F & Voltage-to-Frequency converter \\
CODA & CEBAF Online Data Acquisition (Jefferson lab data acquisition software) \\
EPICS & Experimental Physics and Industrial Control System (Jefferson lab controls software) \\
MCC & accelerator Machine Control Center \\
\hline
\end{tabular}

Table 2.1: Acronyms used in Fig. 2-3 
The high frequency $(1497 \mathrm{MHz})$ signals from the BCMs are converted to $1 \mathrm{MHz}$ signals by two down converters located in the Hall. These signals are then sent to the counting house, where they are filtered by $1 \mathrm{MHz}$ bandpass filters to eliminate noise, amplified and finally measured by two Digital Multi Meters (DMM). The signals from these multi meters and from the Unser multi-meter are fed to the Hall A Controls (HAC) computer, and then through EPICS ${ }^{1}$ to the data stream.

Both sampled and integrated results from the BCMs are recorded in the data stream. Only the sampled data are calibrated against the Unser. The sampled output is generated by digitizing the instantaneous cavity voltage at regular intervals of 4 , 10, and 50 seconds. The integrated data results from converting the cavity output signal to DC and then generating a signal with a frequency proportional to this DC level using a Voltage-to-Frequency (V-to-F) converter. The output pulses are then counted by a scaler unit. All the scaler units are read into the data stream every ten seconds during data acquisition.

Of course, the interesting quantity was the integrated charge for each run. The V-to-F scaler signal provides automatic integration over the run period. However, this integration uses the uncalibrated BCM signal. Therefore the V-to-F signal was calibrated using sampled data, and then was used to determine the integrated charge for this experiment.

\subsubsection{Waterfall target}

The waterfall target used for this experiment was constructed by a group from INFN. The basic design is described in detail in [54], and only a brief outline is presented here. The water foils of the target were formed in a rectangular waterfall chamber placed in the standard Hall A scattering chamber. Three narrow slits were cut out on the roof of the chamber. The water was pumped through a heat exchanger, into a small reservoir above the waterfall chamber. Pressed through the slits and flowing down between stainless-steel poles, the water formed flat thin films which were stable due to the surface tension of the water and the adhesion to the poles.

\footnotetext{
${ }^{1}$ Slow controls software used at Jefferson Lab [52] which controls and communicates with elements such as spectrometer magnets, beam line elements, and beam line equipment.
} 
Some modifications were made to the original design in preparation for the present experiment. The water-foil configuration was redesigned at MIT to minimize energy loss and maximize luminosity for all kinematic settings of the experiment. The waterfall chamber shape was also changed from cylindrical to rectangular.

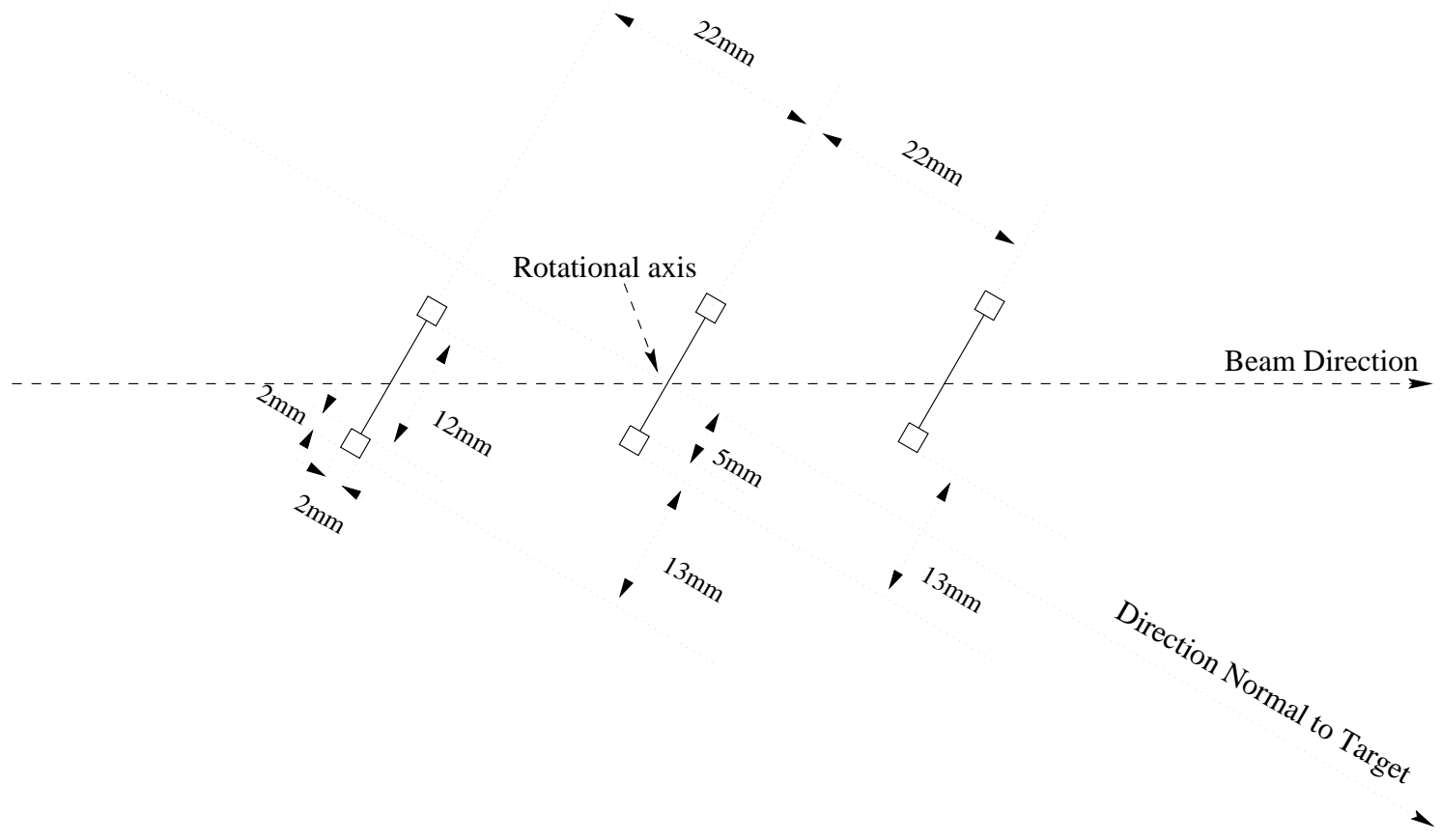

Figure 2-4: Waterfall target foil configuration

For this experiment, a three water-foil configuration was chosen. Each foil was roughly $130 \mathrm{mg} / \mathrm{cm}^{2}$ thick along the beam and was at $30^{\circ}$ to the incident beam. This configuration is better than a single foil three times thicker because the energy loss in the target is reduced. Further, the vertex reconstruction using both spectrometers to the same water-foil helps reduce the accidentals and reconstruct the energy loss for each event. The foil configuration used for this experiment is shown in Fig. 2-4. This configuration was chosen so that the scattered particles do not go through a second water foil for any of the kinematic settings of the experiment. The three foils were identical, each $12 \mathrm{~mm}$ wide and were guided by $2 \mathrm{~mm} \times 2 \mathrm{~mm}$ stainless steel poles.

The beam entrance and exit windows of the waterfall chamber were made from 75 $\mu \mathrm{m} \mathrm{Be}$, while the spectrometer-facing windows were made from $25 \mu \mathrm{m}$ stainless steel. 


\subsubsection{High Resolution Spectrometer (HRS) pair}

The Hall A High Resolution Spectrometers are an identical pair of QQDQ magnetic spectrometers with optical properties that are point-to-point in dispersive direction. Fig. 2-5 shows a cross sectional view of a HRS. The three quadrupoles and the dipole are superconducting magnets.

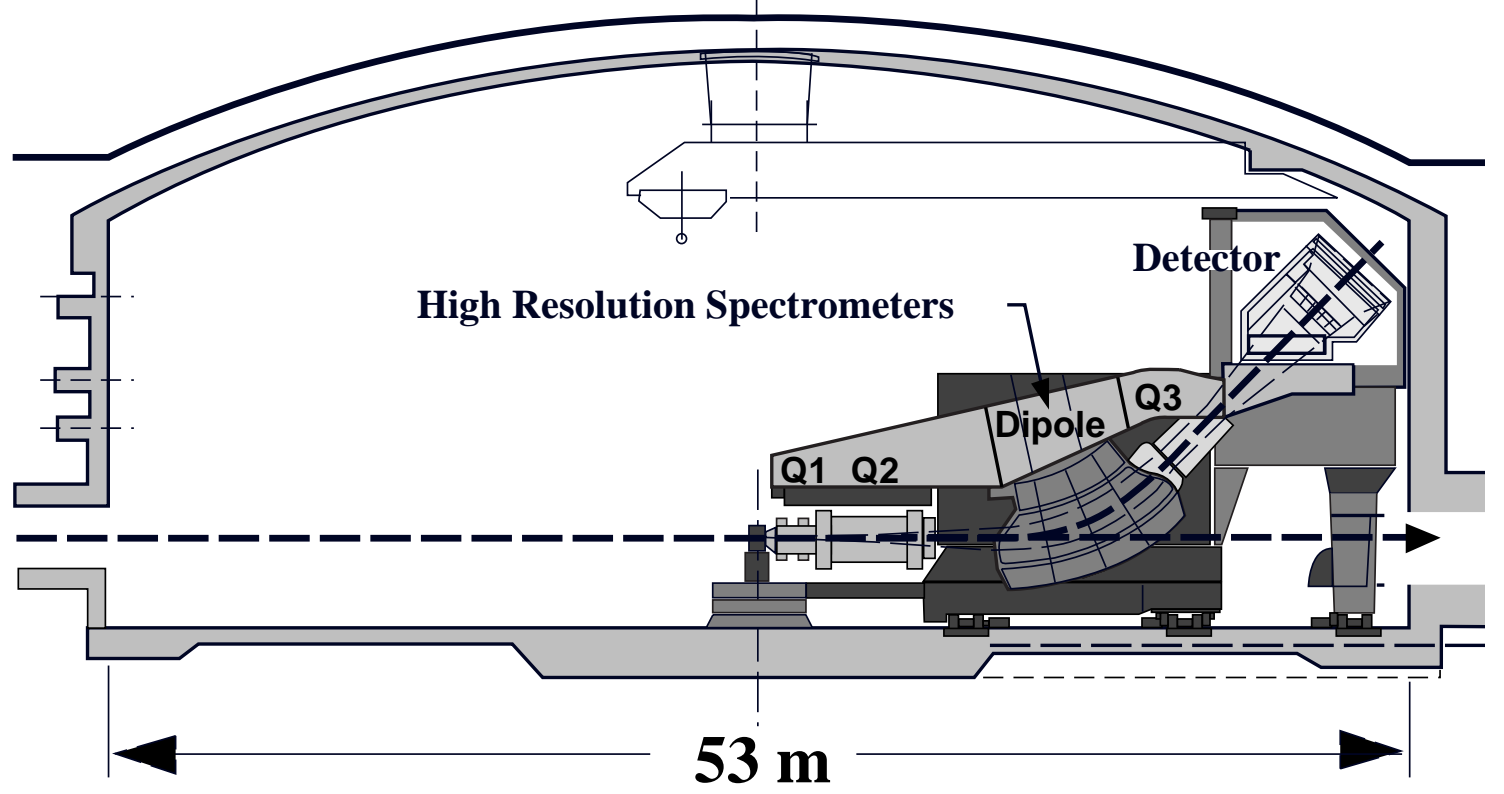

Figure 2-5: A cross sectional view of the experimental Hall A showing the details of an HRS.

Each HRS has a vertical bend of $45^{\circ}$, an optical length of 23.4 , m and a momentum range from $0.3 \mathrm{GeV}$ to $4.0 \mathrm{GeV}$.

The particles traveling through a spectrometer are detected by the focal plane detector package (described in section 2.2.4) placed in the concrete/lead shielding house right above the last quadrupole. The position and angular coordinates of the detected particle tracks are measured by the Vertical Drift Chambers (see section 2.2.7) of the detector package. These "focal plane" coordinates are used to reconstruct the coordinates of the particle track at the interaction vertex in the target. A detailed description of the track reconstruction method and a discussion of the calibration of the HRS pair is given in Appendix A. 


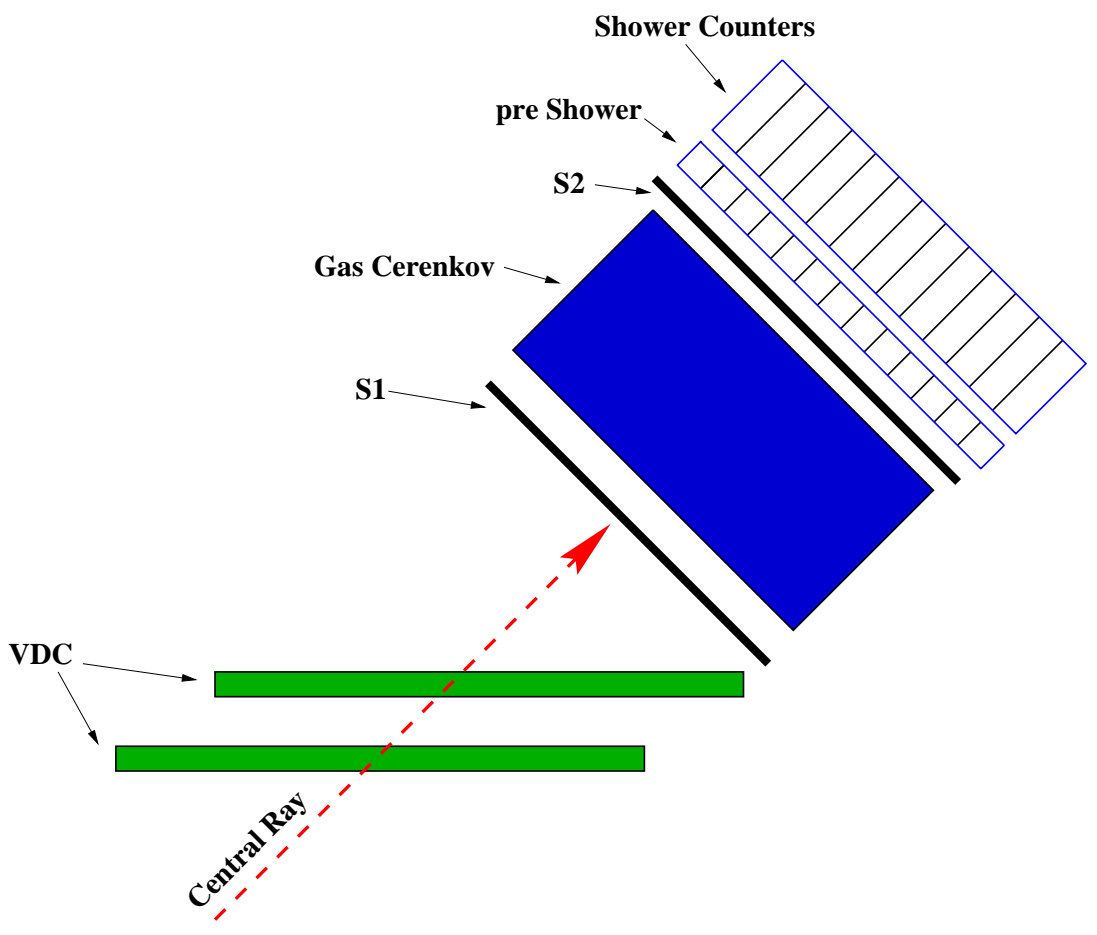

Figure 2-6: Electron arm detector package

\subsubsection{HRS detector packages}

Figs. 2-6 and 2-7 show HRS detector packages. The following components of the electron arm detector package were used for this experiment.

- Two planes of thin scintillators to generate the trigger.

- A gas Cherenkov detector for particle identification.

- Vertical Drift Chambers (VDCs) for the determination of position and angular coordinates of the particle tracks.

Only the VDCs and the trigger scintillators of the hadron arm detector package were used for this experiment.

The following sections present a description of these detectors, followed by discussions of the HRS trigger electronics and the Hall A data acquisition system.

\subsubsection{Scintillators}

The two scintillator planes of a spectrometer detector package are perpendicular to the nominal central ray and are separated by $2.0 \mathrm{~m}$. The surface area of each plane 


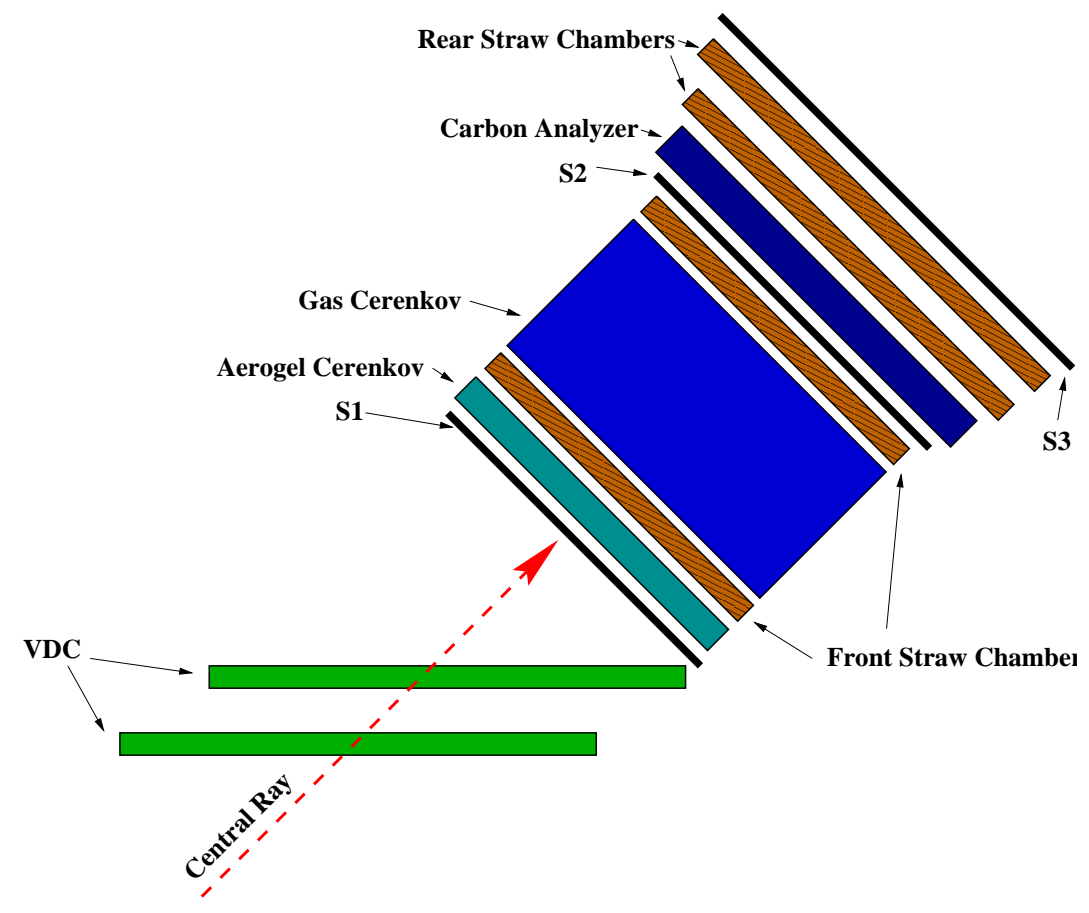

Figure 2-7: Hadron arm detector package

is larger than the particle envelope at the location of the plane. This ensures a high trigger efficiency. Each of the two planes consists of six plastic scintillator paddles. The paddles are arranged in such a way that their long axes are perpendicular to the spectrometer dispersive direction. A $5.0 \mathrm{~mm}$ overlap of the neighboring paddles is used to ensure the complete coverage of the plane. A photo-multiplier tube is mounted on either end of each paddle.

The use of the scintillators to generate the spectrometer triggers is discussed in section 2.2 .8

\subsubsection{Gas Cherenkov counter}

The gas Cherenkov counter in the HRSE detector stack is a rectangular chamber filled with carbon dioxide gas at atmospheric pressure. Five photo-multiplier tubes are mounted on either of the two long sides of the chamber. A detailed description of the Hall A Cherenkov counters is given in the Gas Cherenkov counter section of [55].

A Cherenkov counter is used to differentiate between particles with different masses 
but similar momenta. Particles with velocities exceeding a threshold velocity, $\beta_{\text {thresh }}=$ $1 / n$ where $n$ is the refractive index of the material, produce Cherenkov radiation. In the case of this experiment, the Cherenkov detector was used to differentiate electrons from $\pi^{-}$. For $1 \mathrm{~atm} \mathrm{CO}_{2}, n \sim 1.00041$ resulting in threshold momenta of $17 \mathrm{MeV} / \mathrm{c}$ for electrons and $4.8 \mathrm{GeV} / \mathrm{c}$ for pions.

\subsubsection{Vertical Drift Chambers}

The Vertical Drift Chambers for the HRS pair were constructed, commissioned and installed by the Nuclear Interaction Group from MIT. A brief description of the operation, design and special features of MIT-Hall A VDCs is given here. For furthers detail, see [56], [57], and [58].

Each HRS detector stack is equipped with a VDC package for the detection of particle tracks. A VDC package consists of two VDCs. Figs 2-8 and 2-9 show the positioning of the VDCs near the focal plane of the spectrometer. The lower VDC is located near the ideal focal surface ${ }^{2}$. The second VDC is placed $50 \mathrm{~cm}$ downstream offset in the dispersive direction such that the nominal central ray of the spectrometer passes through the center of each VDC.

\footnotetext{
${ }^{2}$ Note however, that due to an incorrectly mounted sextupole element of the HRS dipole, the actual focal plane does not coincide with the lower VDC.
} 


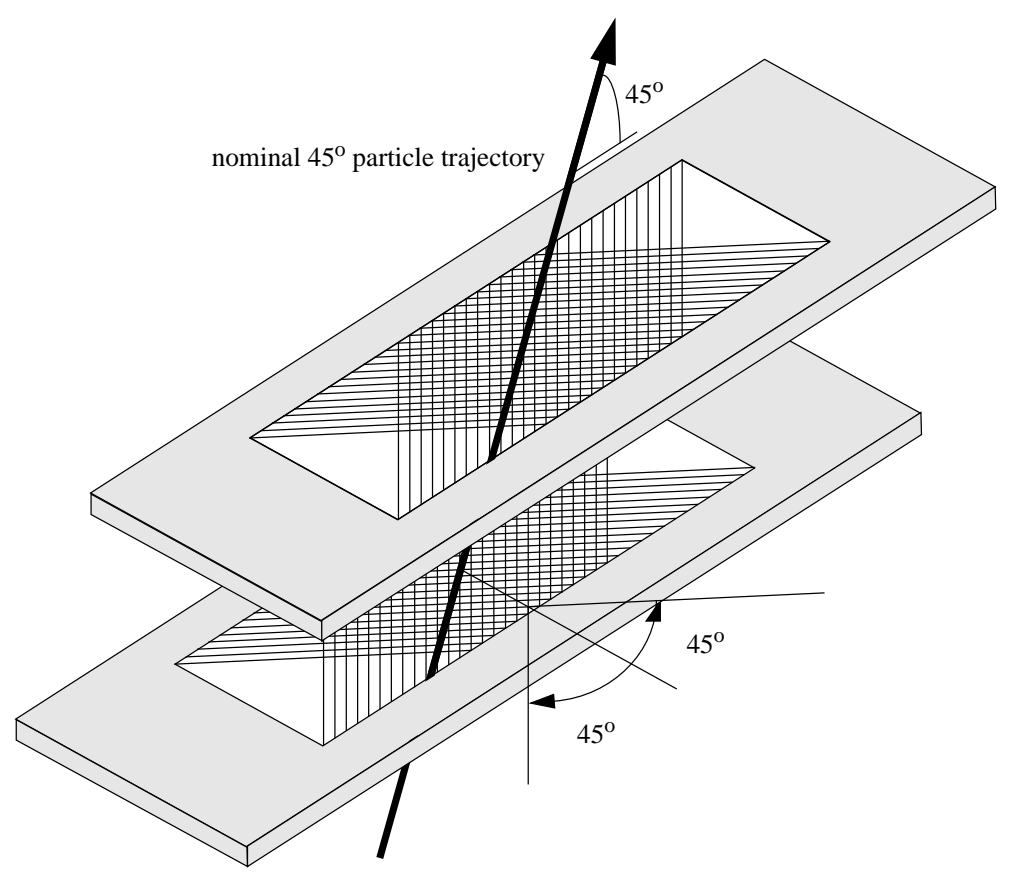

Figure 2-8: Schematic layout of the VDC assembly (not to scale)
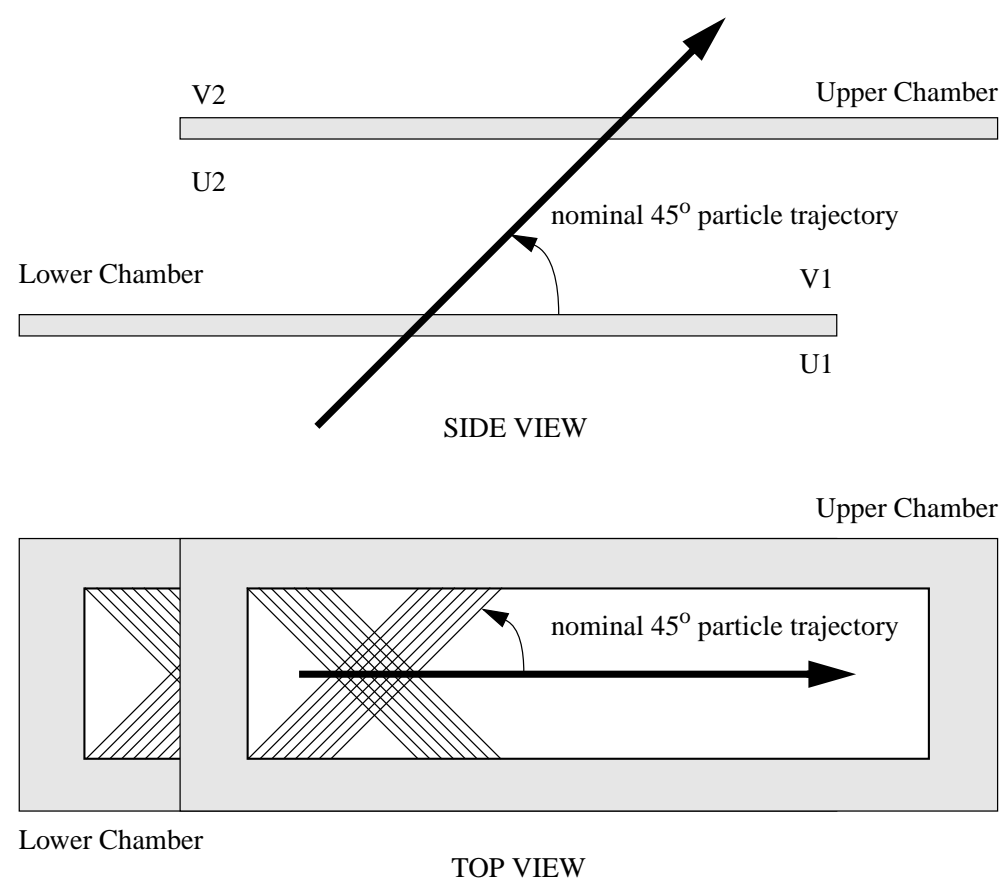

Figure 2-9: Schematic layout of the VDC assembly (not to scale)

A VDC has two wire planes, enabling the detection of both coordinates of a particle track in the plane of the VDC. The position resolution achieved in each direction with 
these VDCs is $\sim 225 \mu \mathrm{m}$ (FWHM). Therefore, the two VDCs separated by $50 \mathrm{~cm}$ are capable of measuring the two angles of the track with a resolution of $0.3 \mathrm{mr}$ (FWHM).

\subsubsection{Principle of operation}

The operational unit of a VDC is a drift cell, made of a thin conducting wire and a conducting plane separated by about $1.3 \mathrm{~cm}$. The surface is kept at a negative high voltage, while the wire is maintained at ground voltage. This results in the electric field configuration as approximately illustrated in Fig. 2-10. Near the wire, the field is radial and increases as $\frac{1}{r}$, while away from the wire the field is parallel and uniform. When a charged particle travels through the chamber, it ionizes the gas inside the chamber and leaves behind a track of electrons and ions along its way. The total number of electron-ion pairs produced depends on the ionization properties of the gas and is proportional to the amount of energy deposited.

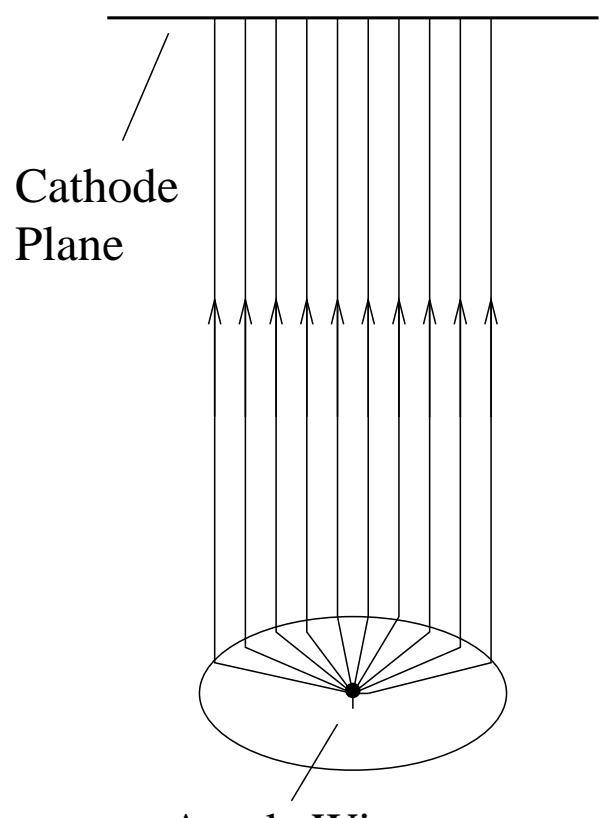

Anode Wire

Figure 2-10: An approximate representation of the electric field configuration of a VDC drift cell. The ellipse represents the locus of transition in the electric field from uniform to radial.

In the electric field of the drift cell, the liberated electrons accelerate towards the wire along the field lines. However, this acceleration does not result in increasing the average drift velocity, as an electron loses the acquired energy at its collisions with 
the gas atoms. In the strong electric field very close to the thin wire, the electrons gather enough energy between two collisions to ionize another gas atom. This creates an avalanche effect, with the number of electrons multiplying at each collision. As the avalanche approaches the wire, the positive ions drift away from the wire. The depletion of positive ions from the vicinity of the wire induces a detectable negative signal on the wire.

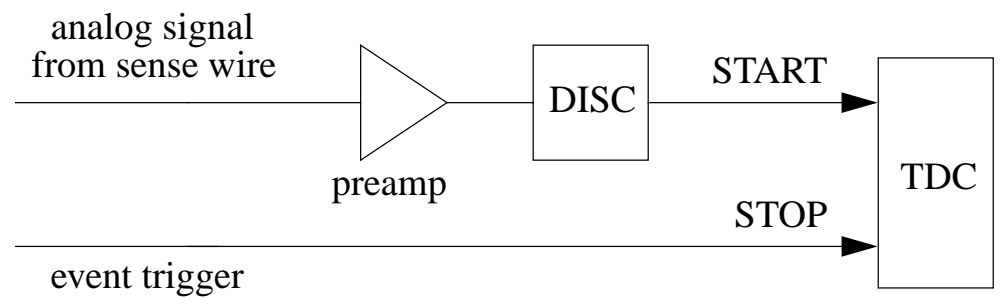

Figure 2-11: A block diagram of the drift time measuring electronics. The wire signal is amplified and then discriminated. The resulting logic pulse is sent to start a Time to Digital converter (TDC) which is stopped by an event trigger from the fast scintillators.

The time elapsed between the initial ionization and the induction of the signal on the sense wire can be measured by using a Time to Digital Converter (TDC) as shown schematically in Fig. 2-11. This drift-time, combined with the knowledge of the drift velocity of the electrons in the chamber gas, yields the drift distance from the particle track to the wire. As shown in Fig. 2-12, the drift distance is different, in general, from the perpendicular distance from the track to the wire plane. For the determination of the intercept of the particle track with the wire plane, the perpendicular distance is a more useful concept than the drift distance. For the MIT, Hall A VDCs, the perpendicular distance is calculated using the method described in reference [56]. 


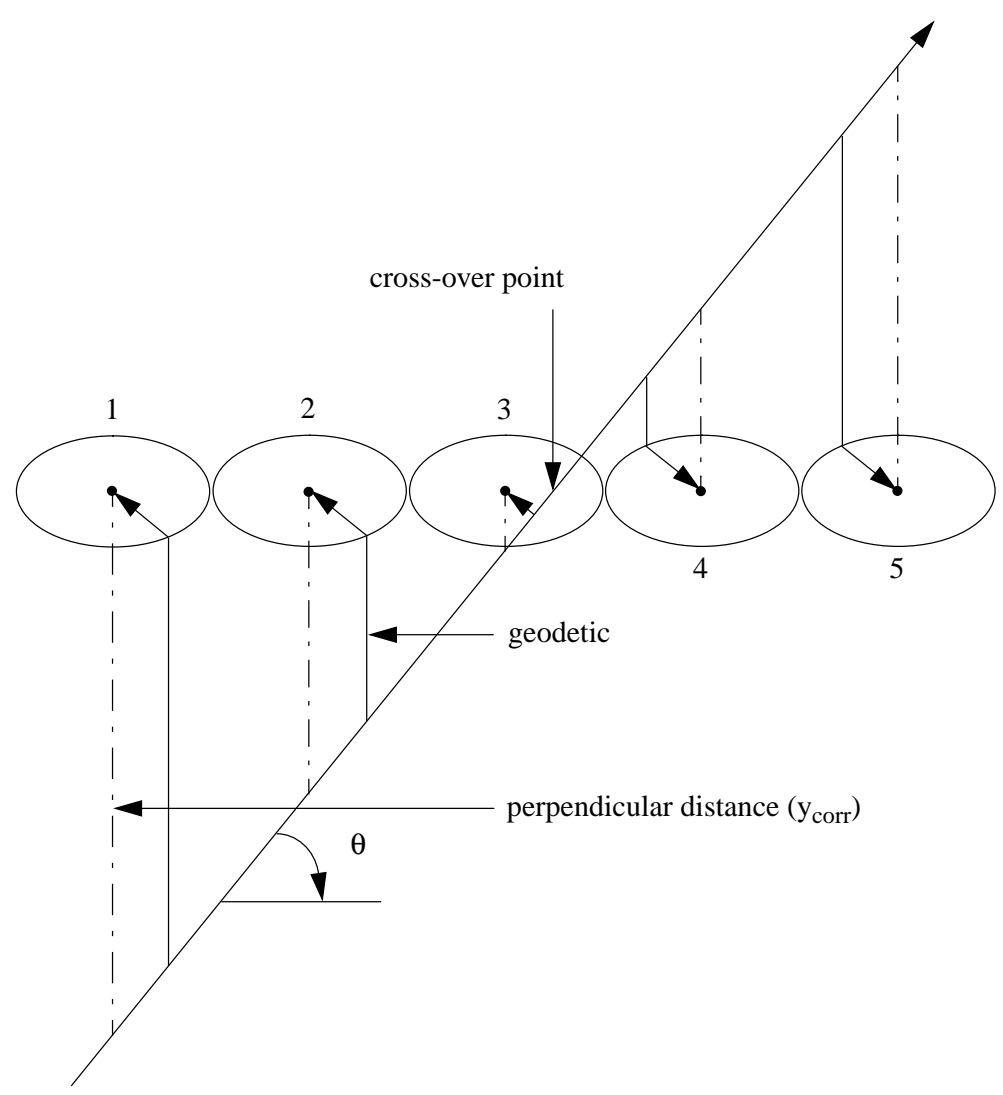

Figure 2-12: A typical track going through five drift cells. The drift paths are the paths of least time (geodetcs) from the track to the wire. The track can be approximately divided into two sections: one in a region of parallel field lines perpendicular to the wire plane and the other in the region near the wire where the field is $1 / \mathrm{r}$ like. The ellipses represent the approximate locus of transition in the electric field from uniform to radial

The MIT Hall A VDCs are of five cell design, i.e a typical $45^{\circ}$ track passes through five drift cells on its way through a wire plane. Therefore, for a typical track, the analysis of drift times yields five perpendicular distances. A fit to these distances gives the intercept of the track with the wire plane. The width of the distribution of uncertainty in the determination of this intercept is defined to be the resolution of the VDC. 


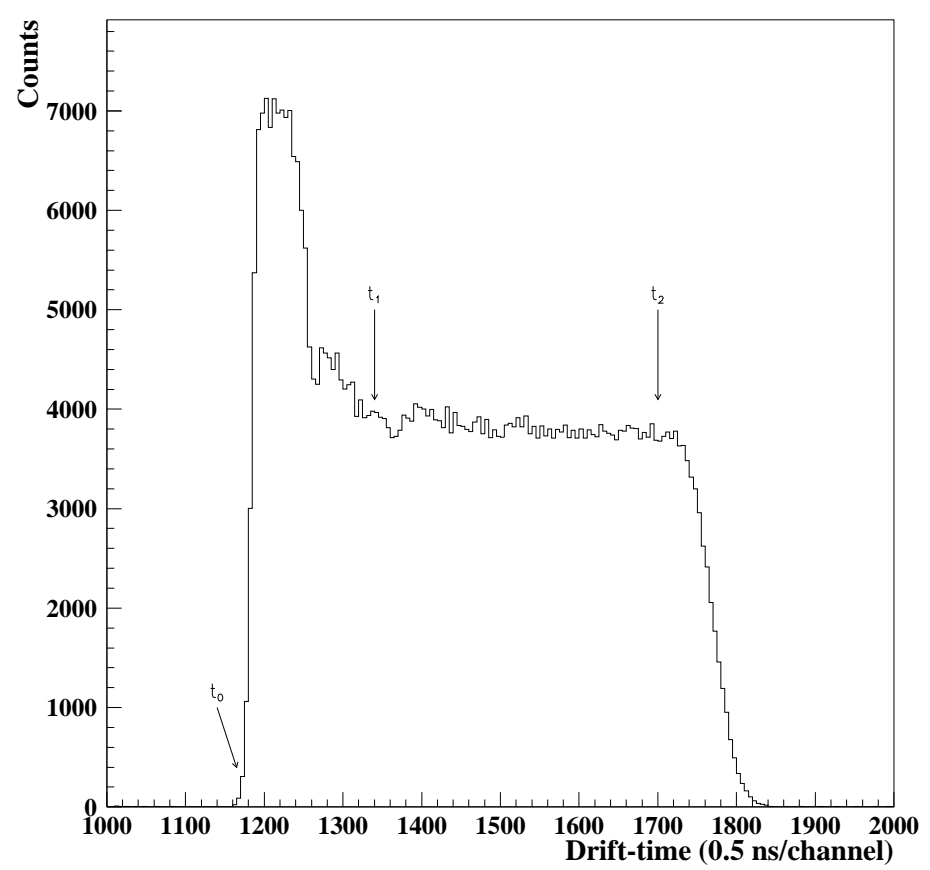

Figure 2-13: A typical drift time spectrum from a MIT Hall A VDC.

Fig. 2-13 shows a typical drift time spectrum obtained from a Hall A VDC. Time $t_{0}$ is the minimum drift time corresponding to tracks grazing the sense wire. The peak in the spectrum is due to tracks passing through the radial region of the electric field. In this region a typical track is roughly perpendicular to the field line along which the electron drifts, whereas in the parallel field region, the tracks are roughly at $45^{\circ}$ to the field lines. As a result, the number of tracks per unit drift length is higher in the radial region, yielding the peak in the spectrum. 


\subsubsection{Physical description of the VDCs}

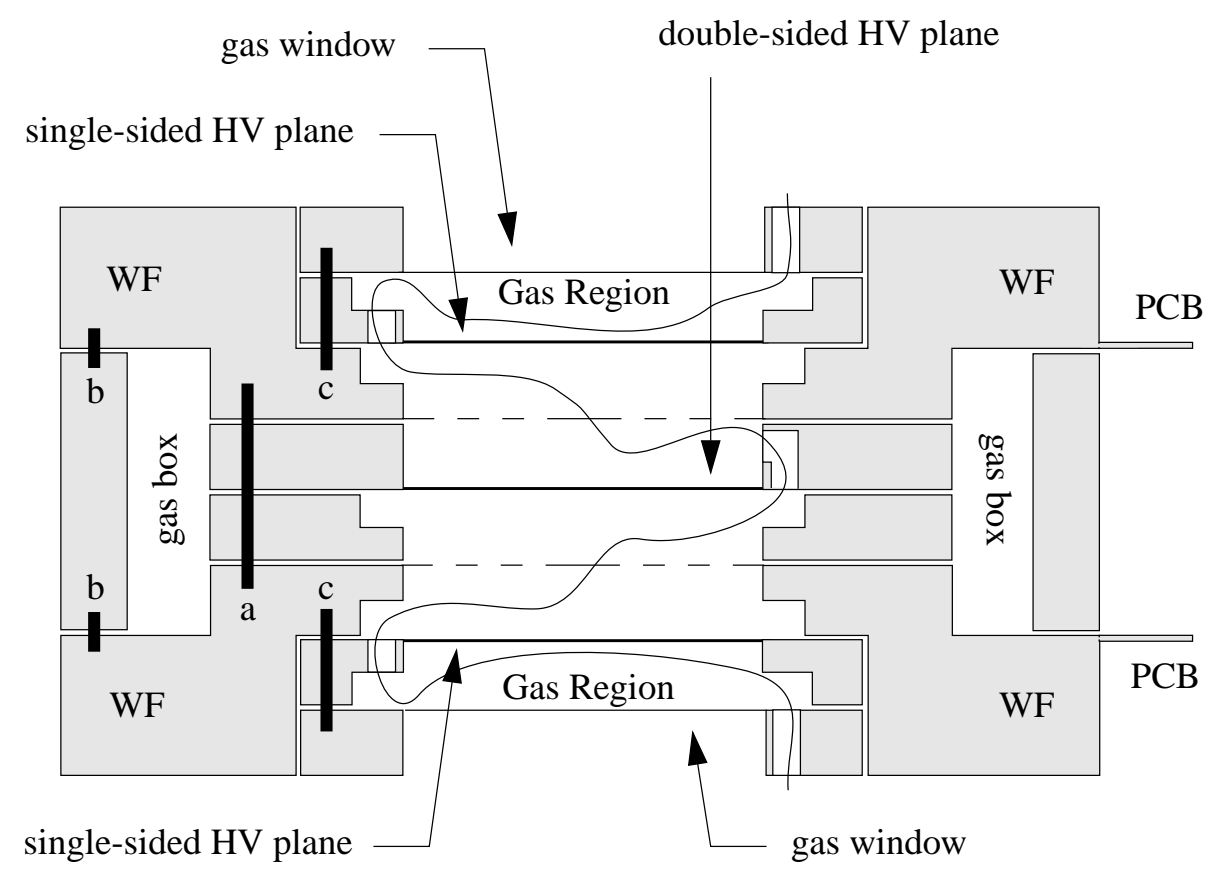

Figure 2-14: The cross sectional view of a VDC (not to scale). Wire frames are labeled "WF" while "PCB" indicates printed circuit boards. The path of the gas follow is shown by the meandering line. The dashed lines indicate the location of the wire planes

Fig. 2-14 shows a cross sectional view of an MIT Hall A VDC. The wire frames are made of the dielectric material stesalite EP-107 (an epoxy-fiberglass composite), with the outside surface copper plated to reduce RF pickup. The high-voltage planes are $6 \mu \mathrm{m}$ gold-plated mylar foils, while the gas windows are $6 \mu \mathrm{m}$ aluminized mylar foils. The wires used for the chambers are $20 \mu \mathrm{m}$, gold-plated tungsten.

The wires on each plane are at $45^{\circ}$ to the edge of the frame, with the wires of the two frames being perpendicular to each other as shown in Fig. 2-9. The $45^{\circ}$ wire orientation ensures that the lengths and the tensions of all the wires are equal.

One unique feature of the MIT Hall A VDCs is the lack of field shaping wires. A conventional VDC has one or more field shaping wires between two signal wires, resulting in narrower drift cells than in the case of an MIT Hall A VDC. As a result, for a given chamber voltage, the amount of charge ending up on a sense wire is not as big as in the present case. Thus a conventional VDC requires a higher chamber voltage to operate. The higher the chamber voltage, the more difficult it is to operate 
the VDC because of spontaneous voltage trips, especially in the humid weather of Virginia. The lack of guard wires in MIT Hall A VDCs has made it possible to operate them at half the cathode voltage used on a conventional VDC. This has resulted in the stable operation of the VDCs with very few voltage trips. These VDCs are operated at $4.0 \mathrm{kV}$ with a chamber gas mixture of $65 \%$ Argon and $35 \%$ Ethane.

Each wire plane contains 368 signal wires. The high resolution of the VDC requires precise positioning of the wires on the frame. The following wire mounting procedure was used in order to achieve this precise positioning of the wires. The grooves for wire mounting were machined on the frame using a precision milling machine. Each wire is placed in a $100 \mu \mathrm{m}$ hole in a thick tube. This tube fits tightly in a sleeve precisely placed in a groove on the wire frame. The wire-tube assembly is mounted on a spring. Fig. 2-15 shows the wire assembly. For a detailed discussion of the wire stringing, see [56]. All the wire positions were measured after stringing, and were found to be within $\pm 50 \mu \mathrm{m}$ of the required positions. 


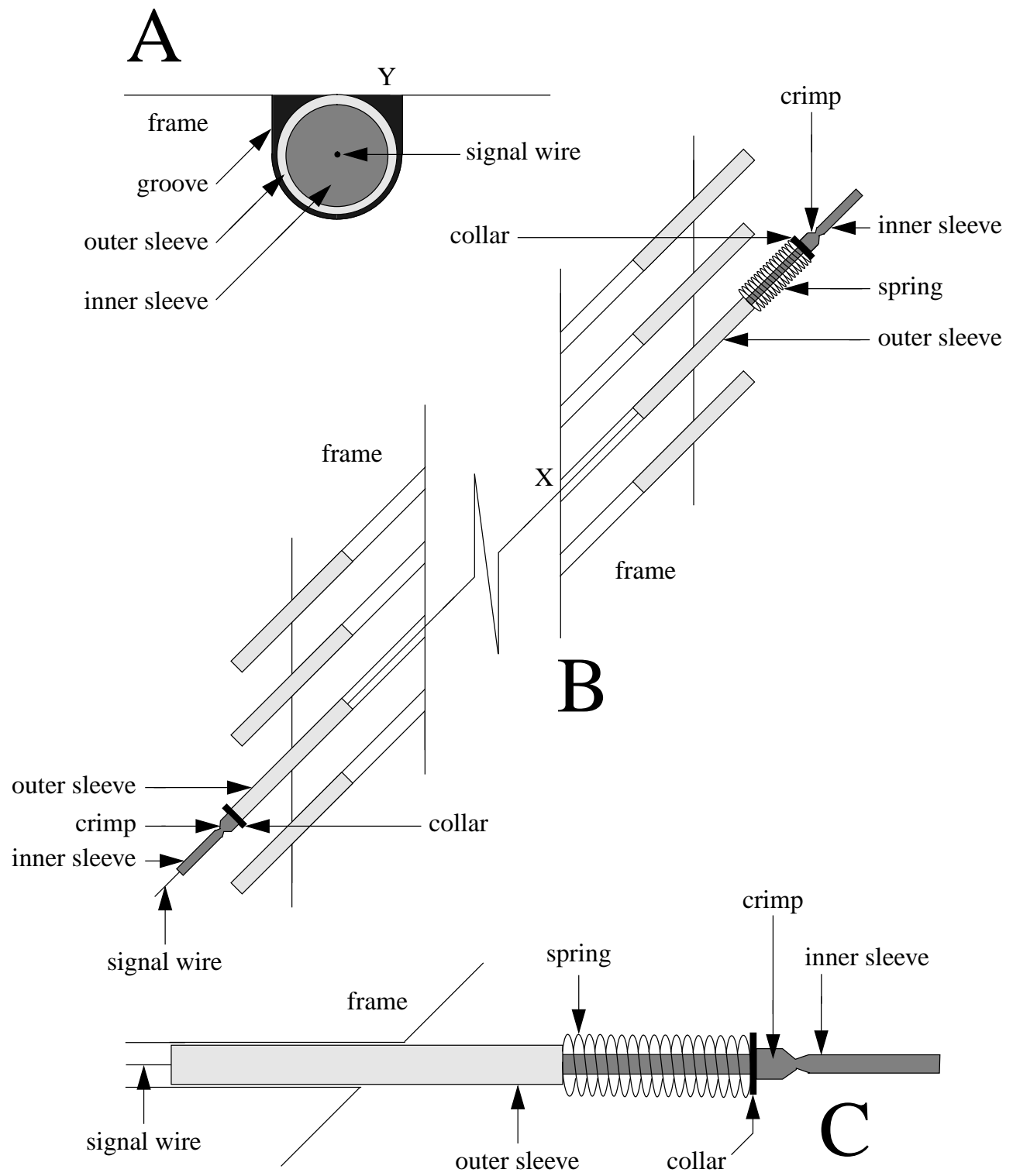

Figure 2-15: VDC wire assembly. Inset 'A' shows a cross sectional view of a single wire in its positioning channel. Inset 'B' presents an overall view of a wire mounted on a wire frame. Inset ' $\mathrm{C}$ ' is a detailed view of the spring-loaded end of a wire. None of the sketches are to scale

\subsubsection{Trigger electronics}

The HRSE trigger detector package consists of (a) two scintillator planes, S1 and S2. (b) a gas Cherenkov counter (c) a lead-glass shower detector, and (d) a lead-glass pre-shower detector. The HRSH trigger detector package includes three scintillator planes, and gas and aerogel Cherenkov counters. The complete trigger configuration on the electron spectrometer and the complete coincidence trigger are presented in 
Appendix C.

For this experiment, only the S1 and S2 scintillator planes on each spectrometer were used to generate the trigger for that spectrometer. Fig. 2-16 shows a simplified schematic diagram of the trigger components used for this experiment.

Each scintillator plane consists of six scintillator paddles. An acceptable hit on a given scintillator paddle requires signals from both left and right photo multiplier tubes of that paddle in coincidence. These signals are then sent to a Memory Lookup Unit (MLU) which can be thought of as a programmable coincidence unit. To generate a singles trigger for the spectrometer, the MLU requires signals from the two scintillator planes in coincidence. Further, the MLU can be programmed to check for hit patterns on the two planes, to make sure that the two hits belong to an approximately $45^{\circ}$ track.

The singles triggers, $\mathrm{S} 1$ from the electron arm and S3 from the hadron arm, are then sent to an overlap AND circuit to form a coincidence trigger, S5. All three trigger types are sent to a scaler unit for counting and to a Trigger Supervisor (TS) unit. The TS can be thought of as a two part device, with a pre-scale function and a MLU function. When a trigger arrives at the TS, the pre-scale function acts first and scales the signal by a factor set by the experimenter. For example, if the pre-scaler factor for trigger type (a) is N, then TS ignores N-1 triggers of type (a) and accepts the $\mathrm{N}^{\text {th }}$ one.

The MLU function of the TS acts on pre-scaled events. Once a trigger arrives, the MLU function checks with the data acquisition system to make sure that it is not busy, or "dead", processing a previous event. If the data acquisition system is "alive", then the TS accepts the trigger and signals to write data. Once a trigger is accepted the data acquisition system is dead for approximately $700 \mu \mathrm{s}^{3}$ and no triggers are accepted during this time. In order to give priority to coincidence events, the singles triggers are delayed by $30 \mathrm{~ns}$ with respect to the coincidence trigger. As a result, in the case of a coincidence, the coincidence trigger is accepted and the singles triggers that generated the coincidence are rejected.

\footnotetext{
${ }^{3}$ This was the situation during this experiment. Improvements to software and hardware since this experiment, have reduced the event dead time to $300 \mu \mathrm{s}$.
} 


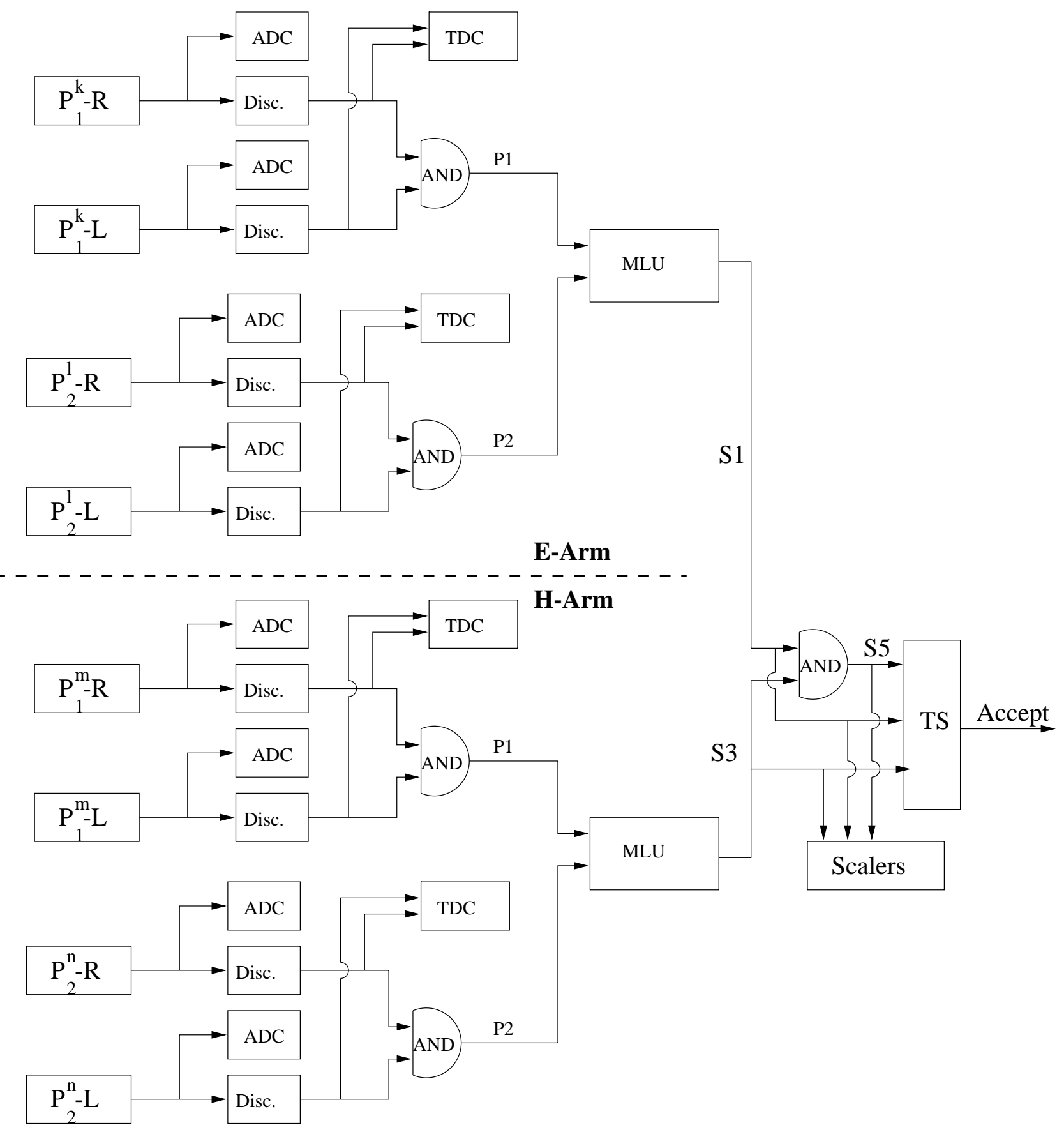

Scintillators

Figure 2-16: A simplified schematic diagram of the trigger setup used for this experiment. Note that none of the delays are shown. $\mathrm{P}_{1(2)}^{i}-\mathrm{R}(\mathrm{L})$ indicates the right (left) photo multiplier tube connected to paddle $i$ of scintillator plane 1(2). For example, $\mathrm{P}_{1}^{2}-\mathrm{R}$ denotes the right photo-multiplier tube connected to paddle 2 of scintillator plane 1. P1(2) indicates the accepted signal from scintillator plane 1(2). S1, S3, and S5 denote electron singles, hadron singles and coincidence triggers respectively. The acronyms used in the figure are described in the text. 


\subsubsection{Hall A data acquisition system}

The Hall A data acquisition system is schematically shown in Fig. 2-17. All Time to Digital Converters (TDC) and Analog to Digital Converters (ADC) used in Hall A are Fastbus based. Fastbus electronics provide higher data acquisition rates than CAMAC. The Jefferson lab data acquisition software package, CODA [53] was used for the data acquisition.

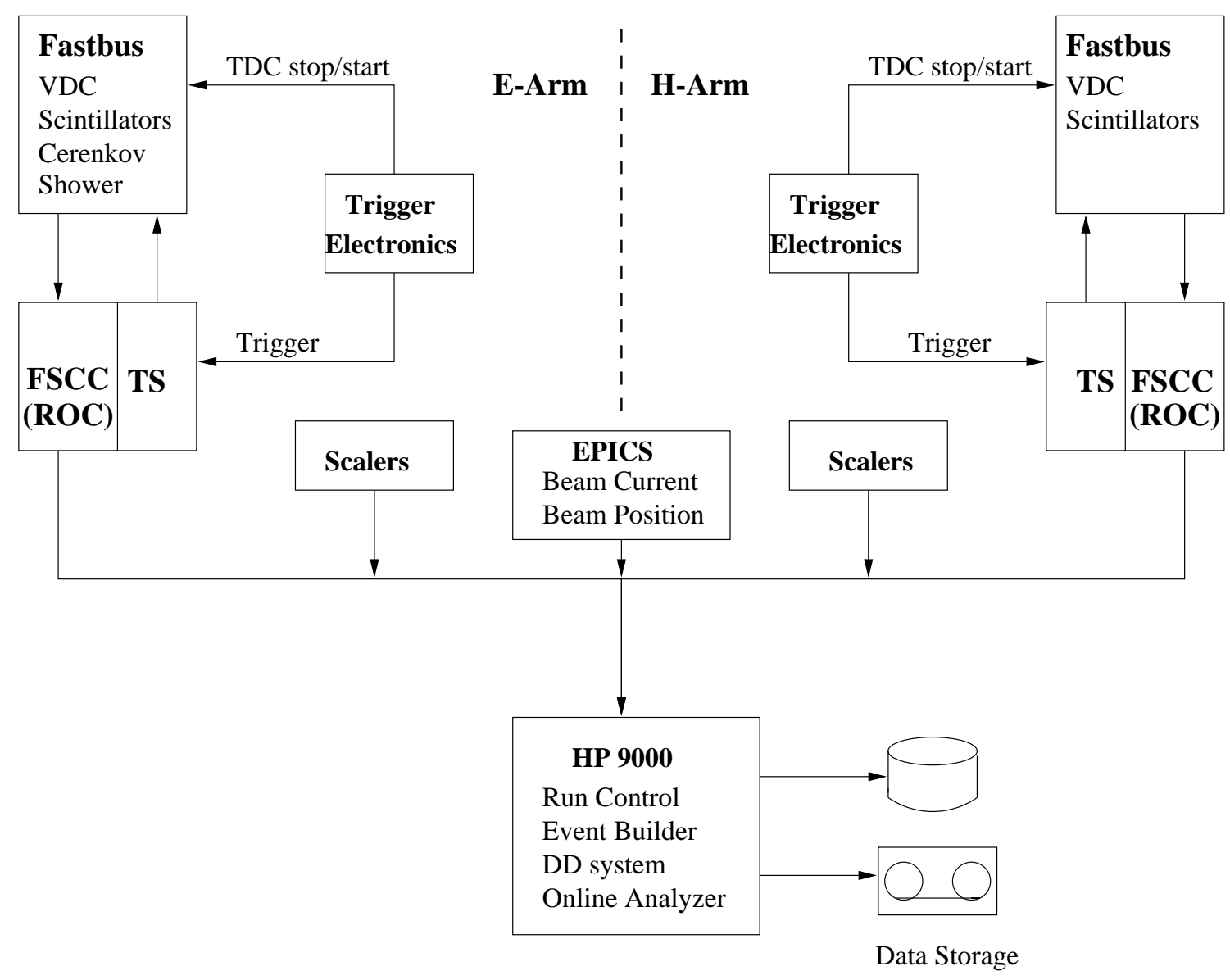

Figure 2-17: A schematic representation of the Hall A data acquisition system used for E89003.

On each spectrometer, a Fastbus crate houses all TDC and ADC modules that register data from focal plane detectors. The Fastbus crate is interfaced with the data acquisition computer via a single board computer which is called the Read Out Controller (ROC) . During E89003, the Fastbus computer used was a Fermilab Smart Crate Controller (FSCC). CODA uses commercially available VxWorks software to communicate with the ROC. 
The ROC transmits data to the data acquisition computer through a dedicated fast Ethernet line. During this experiment, an HP-9000 was used as the data acquisition computer. The CODA user-interface runs on this computer while the event-builder function builds events from the event fragments arriving from each ROC. The data events are written to hard disk and then transfered to tape.

During data acquisition, data can be spied upon using the Data Distribution (DD) system of CODA. The DD system is a shared memory buffer of events in real time. During this experiment, we used Hall A online analyzers dhist and dataspy [61] to analyze data online and to generate diagnostic histograms. These programs rely on the DD system to read data from the data stream.

The scaler modules count the number of triggers of each trigger type, the number of hits on different detectors and other relevant numbers such as the BCM-VtoF frequency. The accumulated number on each scaler module is read into the data stream every ten seconds. The sampled beam current and beam position information as well as the magnet information are written to the data stream through the Jefferson Lab slow controls software, EPICS [52]. 


\subsection{Kinematics and experimental method}

\subsubsection{Quasielastic region}

The kinematics of the ${ }^{16} \mathrm{O}\left(\mathrm{e}, \mathrm{e}^{\prime} \mathrm{p}\right)$ measurements in the quasielastic region are given in table 2.2. All quasielastic measurements were performed at fixed values of momentum and energy transfer, $|\vec{q}|, \omega$, of $1 \mathrm{GeV}$ and $445 \mathrm{MeV}$. The corresponding four momentum transfer squared, given by $Q^{2}=q^{2}-\omega^{2}$, was $0.8(\mathrm{GeV} / \mathrm{c})^{2}$. This combination of $|\vec{q}|, \omega$ and $Q^{2}$ corresponds to a point close to the center of the quasielastic peak as determined by the relationship, $\omega=\frac{Q^{2}}{2 M_{N}}$, where $M_{N}$ is the nucleon mass.

The nominal beam energies used for this experiment, $0.845,1.645$ and $2.445 \mathrm{GeV}$, were chosen to give a broad range of the values of the virtual photon polarization, $\epsilon^{4}$. The range in $\epsilon$ acts as the lever arm in the separation of the longitudinal and transverse response functions. Thus, a broad range in $\epsilon$ results in smaller systematic errors in the separated response functions.

\begin{tabular}{|c|c|c|c|c|}
\hline \hline $\begin{array}{c}\mathrm{E}_{\text {beam }} \\
(\mathrm{GeV})\end{array}$ & $\begin{array}{c}\theta_{e} \\
\left(^{\circ}\right)\end{array}$ & $\begin{array}{c}\theta_{p q} \\
\left(^{\circ}\right)\end{array}$ & kinematics & $\begin{array}{c}\left|\vec{p}_{\text {miss }}\right| \\
(\mathrm{MeV} / \mathrm{c})\end{array}$ \\
\hline 0.845 & 100.7 & 0 & $\|$ & 0 \\
0.845 & 100.7 & 8 & $\perp$ & 140 \\
0.845 & 100.7 & 16 & $\perp$ & 275 \\
\hline 1.645 & 37.17 & -8 & $\perp$ & 140 \\
1.645 & 37.17 & 0 & $\|$ & 0 \\
1.645 & 37.17 & 8 & $\perp$ & 140 \\
\hline 2.445 & 23.38 & -20 & $\perp$ & 350 \\
2.445 & 23.38 & -16 & $\perp$ & 275 \\
2.445 & 23.38 & -8 & $\perp$ & 140 \\
2.445 & 23.38 & 0 & $\|$ & 0 \\
2.445 & 23.38 & 8 & $\perp$ & 140 \\
2.445 & 23.38 & 16 & $\perp$ & 275 \\
2.445 & 23.38 & 20 & $\perp$ & 350 \\
\hline \hline
\end{tabular}

Table 2.2: Quasielastic kinematics

$$
{ }^{4} \epsilon=\frac{\sqrt{V_{T}}}{2 V_{L}}
$$


Fig. 2-18 illustrates the settings of the two spectrometers at the three beam energies. At each beam energy, the HRSE was set at a central angle corresponding to the value of $|\vec{q}|$ given above. The HRSH was initially positioned along $\vec{q}$. This setting corresponds to parallel kinematics with $\hat{q}=\hat{p}_{p}$. 


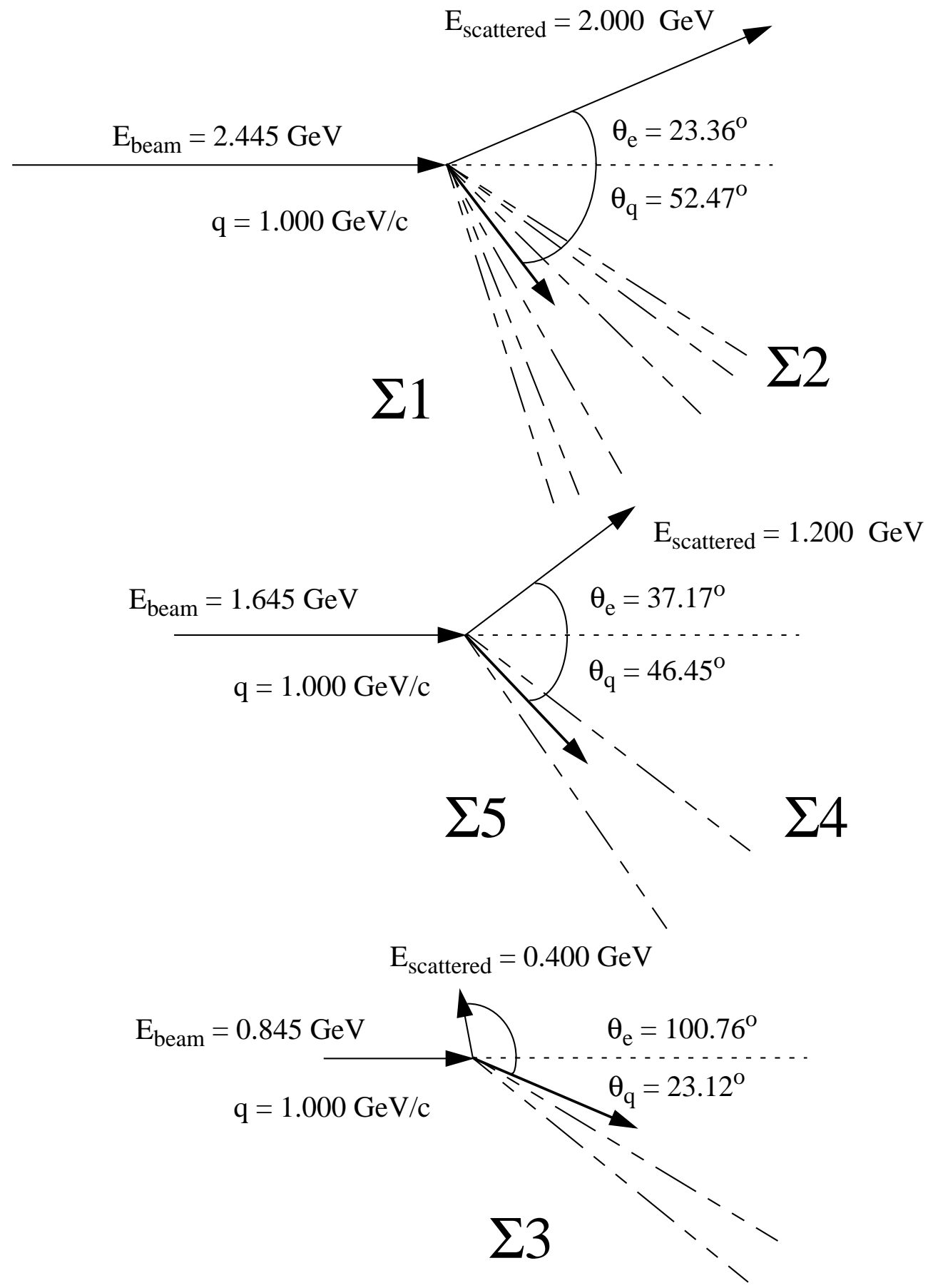

Figure 2-18: A schematic of the experimental settings, indicating the beam energies and the spectrometer central angles.

The accuracy of a response function separation experiment depends on how well the values of $|\vec{q}|$ and $\omega$ can be matched between different kinematic settings corresponding to different values of $\epsilon$. In order to ensure proper matching of $|\vec{q}|$, data were acquired 
in parallel kinematics with a pin-hole collimator on the HRSE prior to the regular data taking. The momentum of the protons detected on the HRSH corresponding to $\mathrm{H}\left(\mathrm{e}, \mathrm{e}^{\prime} \mathrm{p}\right)$ scattering in this case is equal to $|\vec{q}|$. The high momentum resolution of the spectrometer allowed the determination of the $\mathrm{H}\left(\mathrm{e}, \mathrm{e}^{\prime} \mathrm{p}\right)$ momentum peak to the $2 \times 10^{-4}$ level. Thus the pin-hole technique resulted in the matching of $|\vec{q}|$ to the $2 \times 10^{-4}$ level between different kinematic settings.

The regular ${ }^{16} \mathrm{O}\left(\mathrm{e}, \mathrm{e}^{\prime} \mathrm{p}\right)$ data taking was performed with $6.0 \mathrm{msr}$ collimators on both spectrometers. After parallel kinematics data taking at each beam energy, the HRSH was moved to the angles indicated in Fig. 2-18 for perpendicular kinematics data taking.

Throughout the experiment $\mathrm{H}\left(\mathrm{e}, \mathrm{e}^{\prime}\right)$ singles data, measured simultaneously with the ${ }^{16} \mathrm{O}\left(\mathrm{e}, \mathrm{e}^{\prime} \mathrm{p}\right)$ data from the waterfall target, were collected on the HRSE. Thes data provided a continuous monitor of luminosity and beam energy.

\subsubsection{Dip region kinematics}

Data for the dip region were acquired at a beam energy of $1.645 \mathrm{GeV}$. The quantities $\omega$ and $|\vec{q}|$ were kept constant at $589 \mathrm{MeV}$ and $1026 \mathrm{MeV} / \mathrm{c}$ respectively. This combination of $\omega$ and $|\vec{q}|$ corresponds to a point $213 \mathrm{MeV}$ above the center of the quasielastic peak and approximately $87 \mathrm{MeV}$ below the $\Delta$ peak. The variable $y$ (defined to be the minimum value of the nucleon initial momentum for a certain combination of $\omega$ and $\vec{q}$ ) is a better measure of how quasielastic a given kinematic setting is than is the value of $\omega$. For a point on top of the quasielastic peak $y$ is equal to zero. For $\omega$ and $\vec{q}$ corresponding to our dip region kinematics, the value of $y / m_{N}$ (where $m_{N}$ is the nucleon mass) calculated by using the equation [31]

$$
y=-\frac{|\vec{q}|}{2}+\frac{m_{N} \omega}{|\vec{q}|} \sqrt{\frac{\vec{q} \cdot \vec{q}}{Q^{2}}\left(1+\frac{Q^{2}}{4 m_{N}^{2}}\right)}
$$

is 0.22 .

The momentum and the angle of the electron spectrometer were held fixed at values corresponding to the values of $\omega$ and $|\vec{q}|$ given above. The hadron spectrometer was positioned at a central angle corresponding to parallel kinematics. Data were acquired 


\begin{tabular}{|l|l|l|l|l|}
\hline$E_{\text {beam }}$ & $\theta_{e}$ & $P_{e}$ & $\theta_{p}$ & $P_{p}$ \\
\hline \hline $1645 \mathrm{MeV}$ & $37.17^{\circ}$ & $1056 \mathrm{MeV} / \mathrm{c}$ & $38.45^{\circ}$ & $828 \mathrm{MeV} / \mathrm{c}$ \\
\hline & & & & $890 \mathrm{MeV} / \mathrm{c}$ \\
\hline & & & & $957 \mathrm{MeV} / \mathrm{c}$ \\
\hline & & & & $1029 \mathrm{MeV} / \mathrm{c}$ \\
\hline & & & & $1104 \mathrm{MeV} / \mathrm{c}$ \\
\hline & & & & $1190 \mathrm{MeV} / \mathrm{c}$ \\
\hline
\end{tabular}

Table 2.3: Dip region kinematic settings. Note that $\theta_{e}$ and $\theta_{p}$ denote the angles of electron and proton spectrometers respectively while $P_{e}$ and $P_{p}$ denote the central momenta of the two spectrometers.

for six momentum settings of the HRSH. Table 2.3 summarizes the kinematics for this region. 


\section{Chapter 3}

\section{Data Analysis}

\subsection{Analysis of the raw data}

The Hall A event analyzer ESPACE [60] was used to analyze the raw data events for this experiment. ESPACE was written by Dr. Eddy Offerman and was adapted to Jefferson lab Hall A from Mainz.

ESPACE reads in data events in CODA format and decodes them. First it performs detector and obtains the focal plane vertex, $\left(x_{f p}, \theta_{f p}, y_{f p}, \phi_{f p}\right)$. A description of the detector level analysis for this experiment is given in [1]. Using the HRS optics data base, ESPACE then calculates the target vertex $\left(\theta_{t g}, y_{t g}, \phi_{t g}, \Delta p / p\right)$ as described in section A.2. Figs. 3-1 to 3-4 show the reconstruction of the target variables for the data taken during this experiment and during the HRS commissioning just prior to the experiment. The resolutions of the target variables achieved with the HRS pair with a thin ${ }^{12} \mathrm{C}$ target at a beam energy of $843.3 \mathrm{MeV}$ are given below.

- Angle determination accuracy

$$
\begin{array}{ll}
\text { - in-plane: } & \pm 0.2 \mathrm{mrad} \\
\text { - out-of-plane: } & \pm 0.6 \mathrm{mrad}
\end{array}
$$

- Angular Resolution (FWHM)
- in-plane:
$2.0 \mathrm{mrad}$
- out-of-plane:
$6.0 \mathrm{mrad}$ 
- Momentum Resolution (FWHM)

$$
2.5 \times 10^{-4}
$$

- Transverse position determination accuracy

$$
\pm 0.3 \mathrm{~mm}
$$

- Transverse position resolution (FWHM)

$$
3.0 \mathrm{~mm}
$$

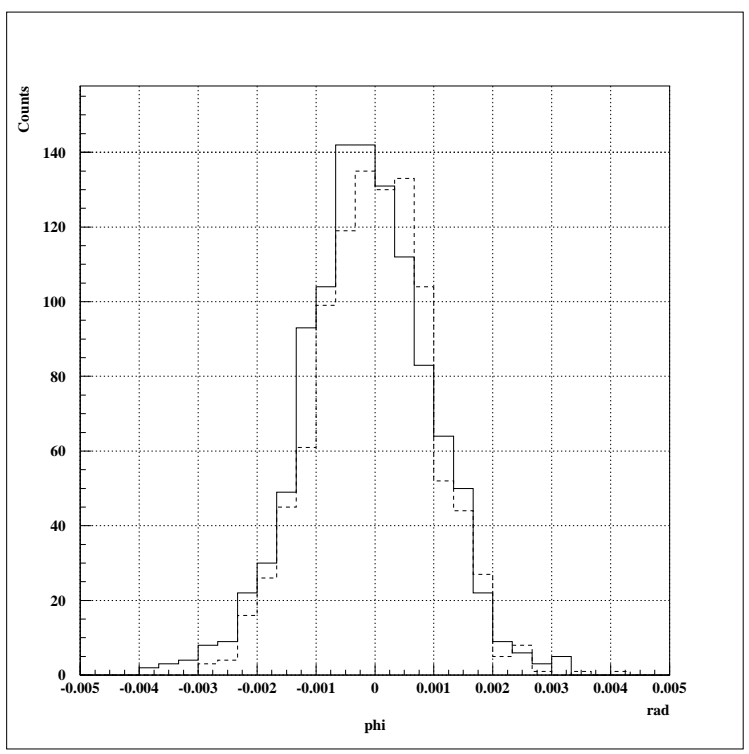

Figure 3-1: Reconstructed $\phi_{t g}$ distribution for a small sieve slit hole $\left(\mathrm{E}_{0}=843.3 \mathrm{MeV}, \theta_{e}=16^{\circ}\right)$. The dashed line shows the simulation of a sieve slit hole folded with a $2.0 \mathrm{mr}$ FWHM Gaussian. 


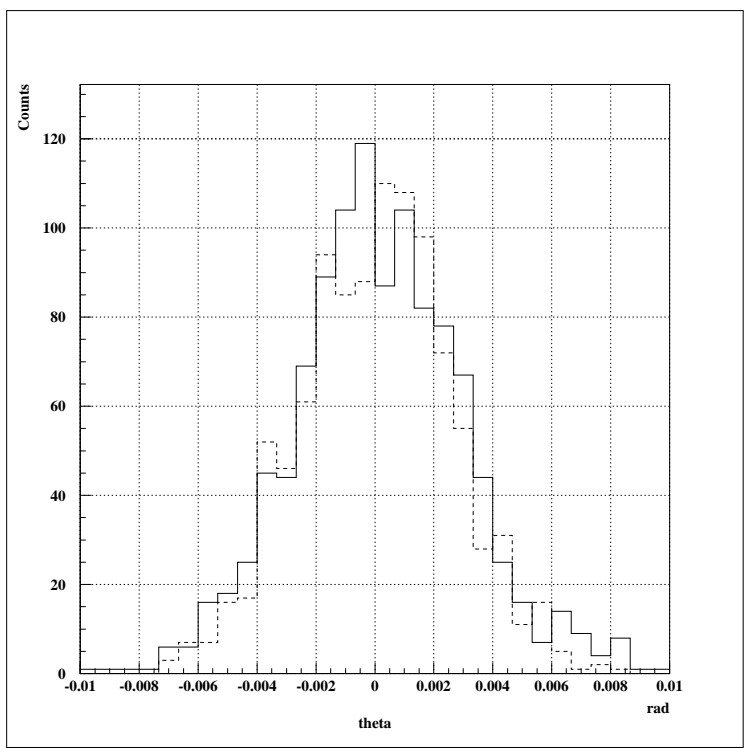

Figure 3-2: Reconstructed $\theta_{t g}$ distribution for a small sieve slit hole $\left(\mathrm{E}_{0}=843.3 \mathrm{MeV}, \theta_{e}=16^{\circ}\right)$. The dashed line shows the simulation of a sieve slit hole folded with a $2.0 \mathrm{mr}$ FWHM Gaussian.

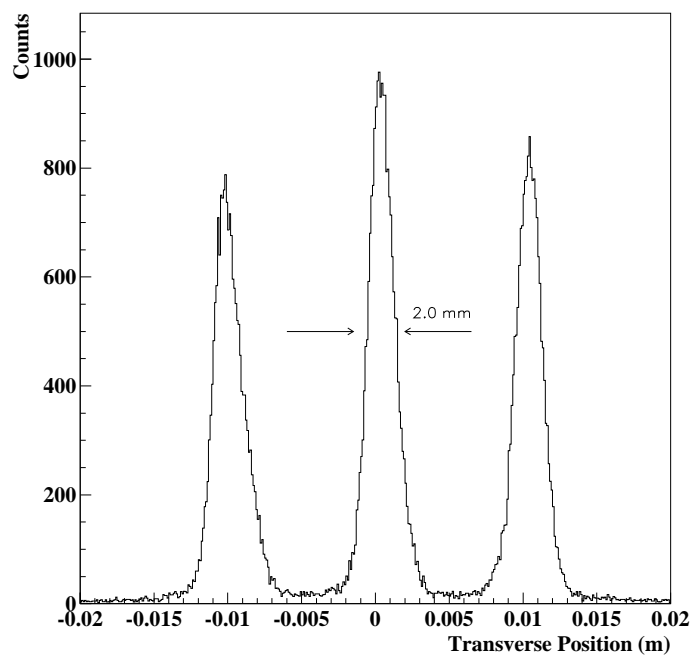

Figure 3-3: Transverse position $\left(y_{t g}\right)$ reconstruction with the waterfall target for $\mathrm{E}_{0}=2442.3 \mathrm{MeV}$ and $\theta_{e}=23^{\circ}$. Note that the actual thickness of a water-foil in $y_{t g}$ direction was approximately 0.5 $\mathrm{mm}$. 


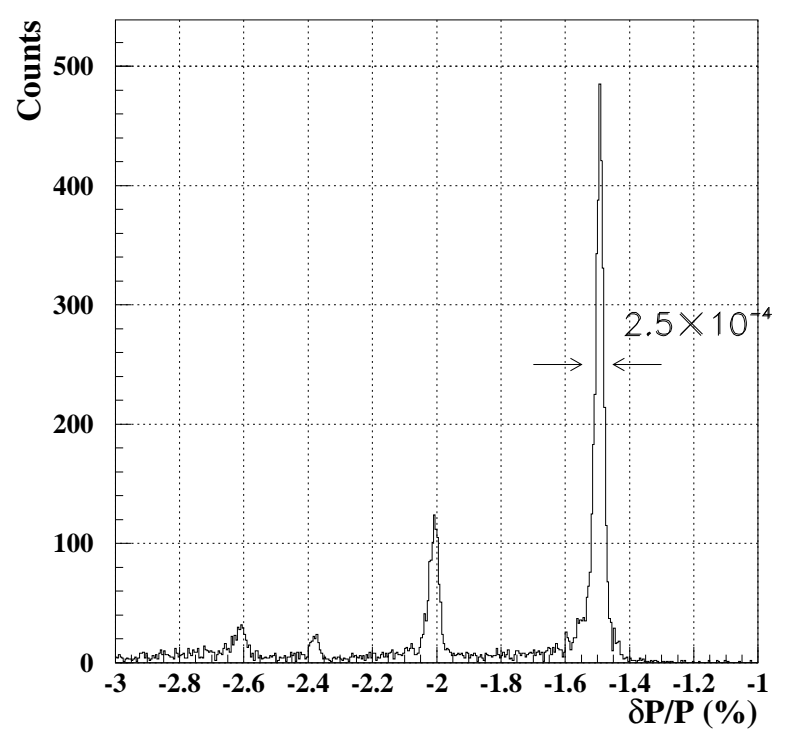

Figure 3-4: $\delta \mathrm{P} / \mathrm{P}$ for ${ }^{12} \mathrm{C}\left(\mathrm{e}, \mathrm{e}^{\prime}\right): \mathrm{E}_{0}=843.3 \mathrm{MeV}, \theta_{e}=16^{\circ}$.

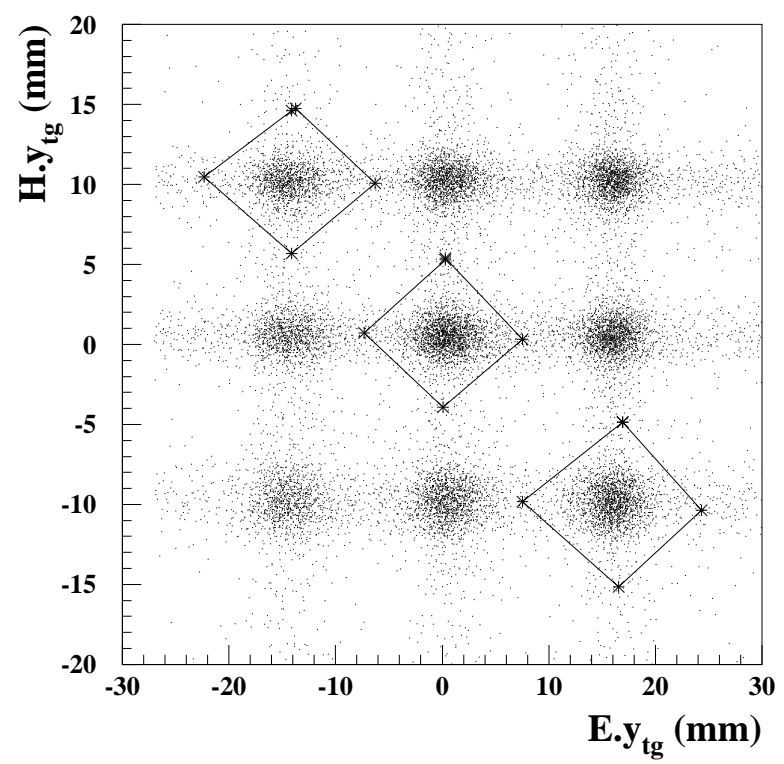

Figure 3-5: $y_{t g}$ reconstructed by the electron spectrometer vs. $y_{t g}$ reconstructed by the hadron spectrometer for $\left(e, e^{\prime} p\right)$ events from the waterfall target. The three boxes indicate the cuts used to select the true coincidence events from the three water-foils. $\left(\mathrm{E}_{0}=2442.3 \mathrm{MeV}, \theta_{p q}=-16^{\circ}\right.$ kinematic setting.)

The high resolution achieved in measuring the transverse position at the target $\left(y_{t g}\right)(\sim 2 \mathrm{~mm}$ FWHM) was used to partially suppress the accidental coincidences by requiring that both spectrometers reconstruct the target vertex to the same water 
foil. Fig. 3-5 presents a scatter plot of $y_{t g}$ reconstructed by the electron spectrometer verses $y_{t g}$ reconstructed by the hadron spectrometer, with the cuts used to select real coincidence events.

Once the target vertex is calculated, $\Delta p / p$ and the spectrometer dipole field $B$, along with the spectrometer constant $\Gamma$, were used to calculate the absolute momentum for the event as measured by the spectrometer.

$$
P_{f}=\Gamma B\left[1+\frac{\Delta p}{p}\right]
$$

For a detailed discussion of the determination of $\Gamma$ for the HRS pair, see [62].

\subsection{Beam energy measurement}

At the time of this experiment none of the standard Hall A beam energy measuring devices were operational. As a result, other methods were used to obtain the beam energy during this experiment. Only a brief outline of these methods is given here. See reference [62] for a detailed discussion of the Hall A beam energy measurement.

The following three methods were used for the beam energy measurement.

- ${ }^{12} \mathbf{C}\left(\mathbf{e}, \mathbf{e}^{\prime}\right)$ technique. This method is based on the accurate knowledge of the locations of states in the ${ }^{12} \mathrm{C}\left(\mathrm{e}, \mathrm{e}^{\prime}\right)$ spectrum. For ultra-relativistic electrons, the energy of the scattered electrons $E_{f}$ can be expressed in terms of the focal plane position as

$$
E_{f}=P_{f}=\Gamma B\left[1+\sum_{i=1}^{2} d_{i} x_{f p}^{i}\right]
$$

where $d_{i}(i=1,2)$ are spectrometer dispersion coefficients, and $\Gamma, B$ and $P_{f}$ are as defined before.

For each state of the ${ }^{12} \mathrm{C}\left(\mathrm{e}, \mathrm{e}^{\prime}\right)$ spectrum, the energy $E_{f}$ can be expressed in terms 
of the excitation energy $E_{x}$ and the beam energy $E_{i}$ as

$$
E_{f}=\frac{E_{i}-E_{l o s s 1}-E_{x}\left[1+\frac{E_{x}}{2 M_{t}}\right]}{f_{r e c}}-E_{l o s s 2},
$$

where

$M_{t}$ is the mass of the target nucleus,

$f_{\text {rec }}$ is the recoil factor given by $f_{\text {rec }}=1+2\left(E_{i}-E_{\text {loss1 } 1}\right) \sin ^{2}(\theta / 2) / M_{t}$,

$E_{l o s s 1}$ and $E_{l o s s 2}$ are mean energy losses before and after scattering.

For the kinematics used for the HRS commissioning $\left(E_{i}=843.3 \mathrm{MeV}\right.$, and $\left.\theta_{e}=16^{\circ}\right), f_{r e c}$ is very insensitive to both the beam energy and the scattering angle [62]. Thus, $f_{\text {rec }}$ calculated using the nominal value of $E_{i}$ is a very good first approximation to be used in eqn. 3.3. The calculation was performed iteratively where the value of $f_{\text {rec }}$ used at each step was calculated using $E_{i}$ determined in the previous step.

Data were acquired for the ${ }^{12} \mathrm{C}\left(\mathrm{e}, \mathrm{e}^{\prime}\right)$ reaction with the elastic peak moved to several different positions across the focal plane by varying the dipole field $B$. At each of the magnetic field excitations, the focal plane positions $x_{f p}$ of the elastic peak and of the excied states were measured. Then, a global least-squares fit was performed on 3.2 and 3.3 to obtain $\Gamma, d_{i}(i=1,2)$, and the beam energy $E_{i}$.

- ${ }^{16} \mathrm{O}\left(\mathbf{e}, \mathbf{e}^{\prime} \mathbf{p}\right)$ missing energy technique. Combined with the spectrometer constant $\Gamma$ calculated using the ${ }^{12} \mathrm{C}\left(\mathrm{e}, \mathrm{e}^{\prime}\right)$ method, the missing energy for the $P_{1 / 2}$ state of the ${ }^{16} \mathrm{O}\left(\mathrm{e}, \mathrm{e}^{\prime} \mathrm{p}\right){ }^{15} \mathrm{~N}$ reaction can be used to calculate the beam energy. This method was used throughout this experiment to monitor the changes in beam energy.

- $\mathbf{H}\left(\mathbf{e}, \mathbf{e}^{\prime} \mathbf{p}\right)$ scattering angle method. For the $H\left(e, e^{\prime} p\right)$ reaction the beam energy can be related to the proton mass $M_{p}$, the electron scattering angle $\theta_{e}$ and the proton scattering angle $\theta_{p}$ by

$$
E_{i}=M_{p}\left[\cot \frac{\theta_{\mathrm{e}}}{2} \cot \theta_{\mathrm{p}}-1\right]+E_{\text {loss } 1} .
$$


This method is independent of the other two techniques. However, the measured beam energy is very sensitive to the knowledge of the spectrometer angles. As a result, a comparison of the beam energy calculated using this method to the beam energy calculated using the other methods can be used to deduce the accuracy of the spectrometer angle measurements. This proved rather useful for this experiment, since the accuracy of this experiment heavily depends on the accuracy of spectrometer angle measurements.

\begin{tabular}{|c|c|c|c|c|}
\hline \hline $\begin{array}{c}\text { Nominal } \\
(\mathrm{MeV})\end{array}$ & $\begin{array}{c}\text { From }{ }^{12} \mathrm{C}\left(\mathrm{e}, \mathrm{e}^{\prime}\right) \\
(\mathrm{MeV})\end{array}$ & $\begin{array}{c}\text { From H(e,e'p) } \\
(\mathrm{MeV})\end{array}$ & $\begin{array}{c}\text { From }{ }^{16} \mathrm{O}\left(\mathrm{e}, \mathrm{e}^{\prime} \mathrm{p}\right) \\
(\mathrm{MeV})\end{array}$ & $\begin{array}{c}\Delta \theta \\
(\mathrm{mrad})\end{array}$ \\
\hline 843.3 & $843.2 \pm 1.2$ & $842.7 \pm 0.1$ & $843.3 \pm 1.2 \pm 0.2$ & 0.3 \\
\hline 1642.5 & $\mathrm{~N} / \mathrm{A}$ & $1644.1 \pm 0.1$ & $1642.5 \pm 2.3 \pm 0.3$ & -0.3 \\
\hline 2442.3 & $\mathrm{~N} / \mathrm{A}$ & $2439.9 \pm 0.2$ & $2442.3 \pm 3.4 \pm 0.5$ & 0.3 \\
\hline \hline
\end{tabular}

Table 3.1: A Comparison of beam energies from the presented methods.

Table 3.1 presents the beam energies determined using the three different methods. Note that the uncertainties quoted for the $\mathrm{H}\left(\mathrm{e}, \mathrm{e}^{\prime} \mathrm{p}\right)$ method do not include the uncertainties arising from the angle measurements. $\Delta \theta$ is the maximum discrepancy corresponding to the beam energies calculated from $\mathrm{H}\left(\mathrm{e}, \mathrm{e}^{\prime} \mathrm{p}\right)$ and ${ }^{16} \mathrm{O}\left(\mathrm{e}, \mathrm{e}^{\prime} \mathrm{p}\right)$ methods. For this experiment, the beam energies were calculated with an accuracy of $1.5 \times 10^{-3}$ using the combination of ${ }^{12} \mathrm{C}\left(\mathrm{e}, \mathrm{e}^{\prime}\right)$ and ${ }^{16} \mathrm{O}\left(\mathrm{e}, \mathrm{e}^{\prime} \mathrm{p}\right)$ methods. Using the ${ }^{16} \mathrm{O}\left(\mathrm{e}, \mathrm{e}^{\prime} \mathrm{p}\right)$ beam energy monitoring, it was established that the beam energy shift during the entire experiment was less than $2.0 \times 10^{-4}$. The agreement between the beam energies calculated with the $\mathrm{H}\left(\mathrm{e}, \mathrm{e}^{\prime} \mathrm{p}\right)$ and ${ }^{16} \mathrm{O}\left(\mathrm{e}, \mathrm{e}^{\prime} \mathrm{p}\right)$ methods implies that the systematic uncertainty for the spectrometer angle measurement is less than 0.3 mrad.

\subsection{Coincidence Time-of-Flight (TOF) calculation}

For a coincidence event, the time between the two spectrometer triggers corresponds to the difference in the flight times through the spectrometers of the detected electron and the proton. Since the particles detected in this experiment were relativistic and the range of the proton momenta was narrow, the time-of-flight for a true coincidence 
event should lie in a narrow range. Fig. 3-6 shows an uncorrected time-of-flight spectrum measured by a TDC with the start and stop signals from the electron trigger and the hadron trigger respectively. The peak of the TOF spectrum represents true coincidence events while the background corresponds to accidental coincidence events for which electron and hadron singles triggers just happened to fall within the coincidence time window.

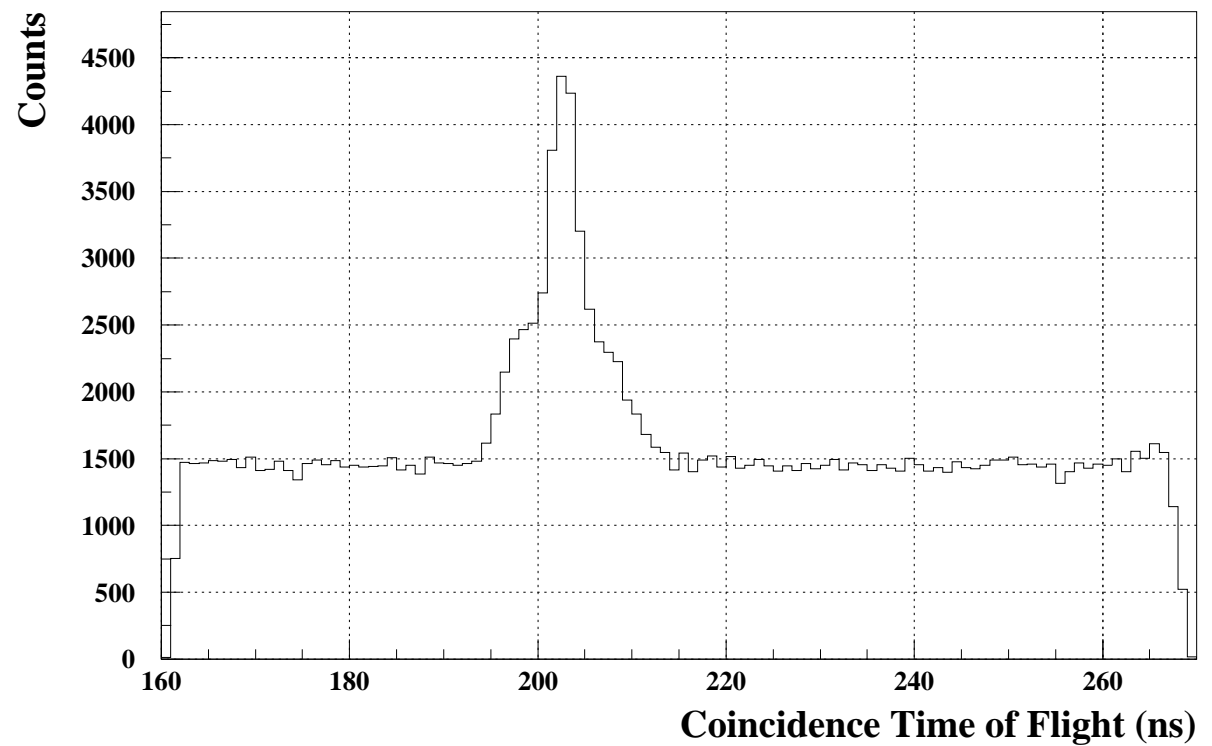

Figure 3-6: Uncorrected coincidence time-of-flight spectrum. $\left(\mathrm{E}_{0}=2442.3 \mathrm{MeV}, \theta_{p q}=-16^{\circ}\right.$. kinematic setting).

The coincidence TOF peak is widened due to the effects described below. These effects are corrected by ESPACE.

- Scintillator timing offset effects. Different scintillator paddles have different time offsets which need to be calculated and subtracted from the raw TOF.

- Variations in proton flight time due to variations of proton velocity. This effect is corrected by using the proton velocity obtained from the flight time between the two scintillator planes.

- Variations in electron and proton flight times due to path differences through the spectrometer. The path difference is essentially a function of the focal plane coordinates $\left(x_{f p}, y_{f p}, \theta_{f p}, \phi_{f p}\right)$, and can be determined from spectrometer optics. 
The correction term $\Delta t$ to TOF due to the last two effects can be calculated as follows.

For a particle traveling along the central ray of the spectrometer, the flight time is given by

$$
t_{0}=\frac{l_{0}}{\beta_{0}},
$$

where $l_{0}$ is the path length along the central ray and $\beta_{0}$ is the velocity of a particle traveling along the central ray.

Due to the last two effects described above, a particle, in general, would have a flight time

$$
t=\frac{l_{0}+\Delta l}{\beta}
$$

where $\beta$ is the velocity of the particle calculated using the flight time between the two scintillator planes and $\Delta l$ is the path difference calculated from spectrometer optics.

Therefore, the correction term can be written as

$$
\Delta t=t-t_{0}=l_{0}\left(\frac{1}{\beta}-\frac{1}{\beta_{0}}\right)+\frac{\Delta l}{\beta} .
$$

The corrections to the raw coincidence TOF spectrum narrows the true coincidence peak to less than 1 ns (FWHM). Fig. 3-7 shows the corrected coincidence time-offlight spectrum. The number of accidental coincidence events that show up as the background in this spectrum can be reduced using the $y_{t g}$ vertex cut described in section 3.1. Fig. 3-8 shows the TOF spectrum after the $y_{t g}$ vertex cut has been applied. 


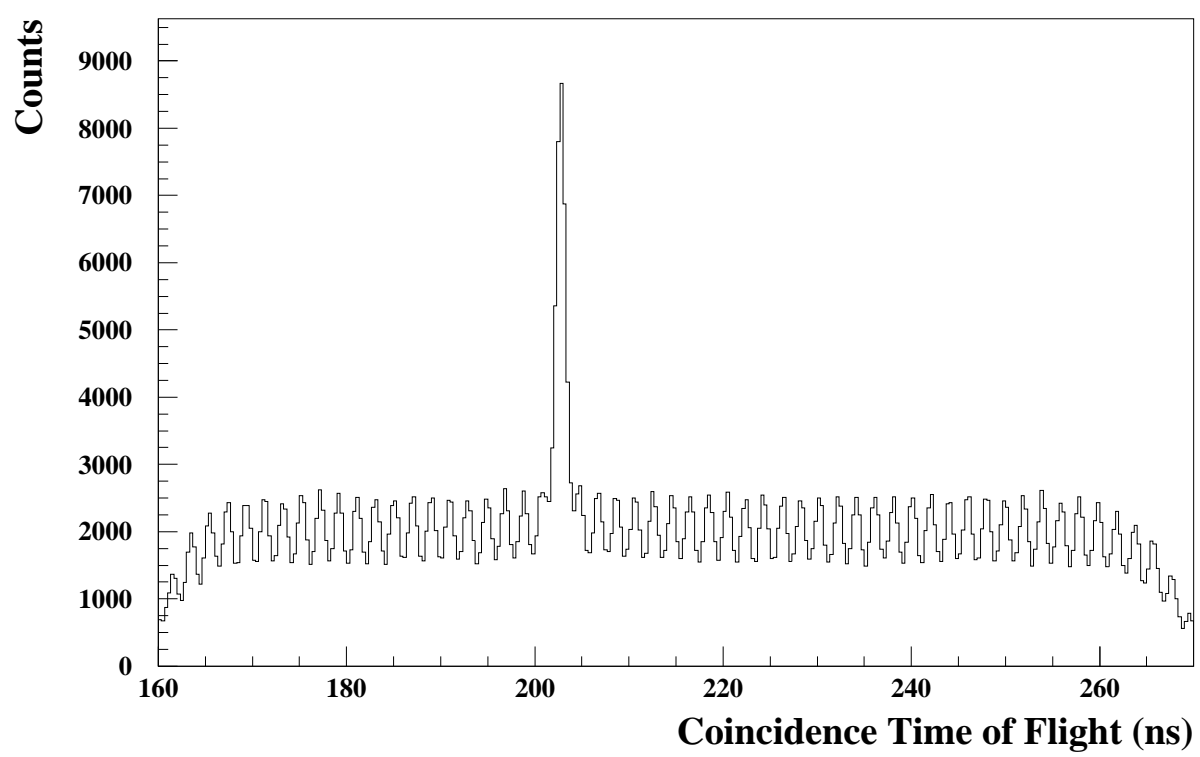

Figure 3-7: Corrected coincidence time-of-flight spectrum. Note the 2 ns pulse structure due to the $500 \mathrm{MHz}$ micro structure of the electron beam. ( $\mathrm{E}_{0}=2442.3 \mathrm{MeV}, \theta_{p q}=-16^{\circ}$ kinematic setting).

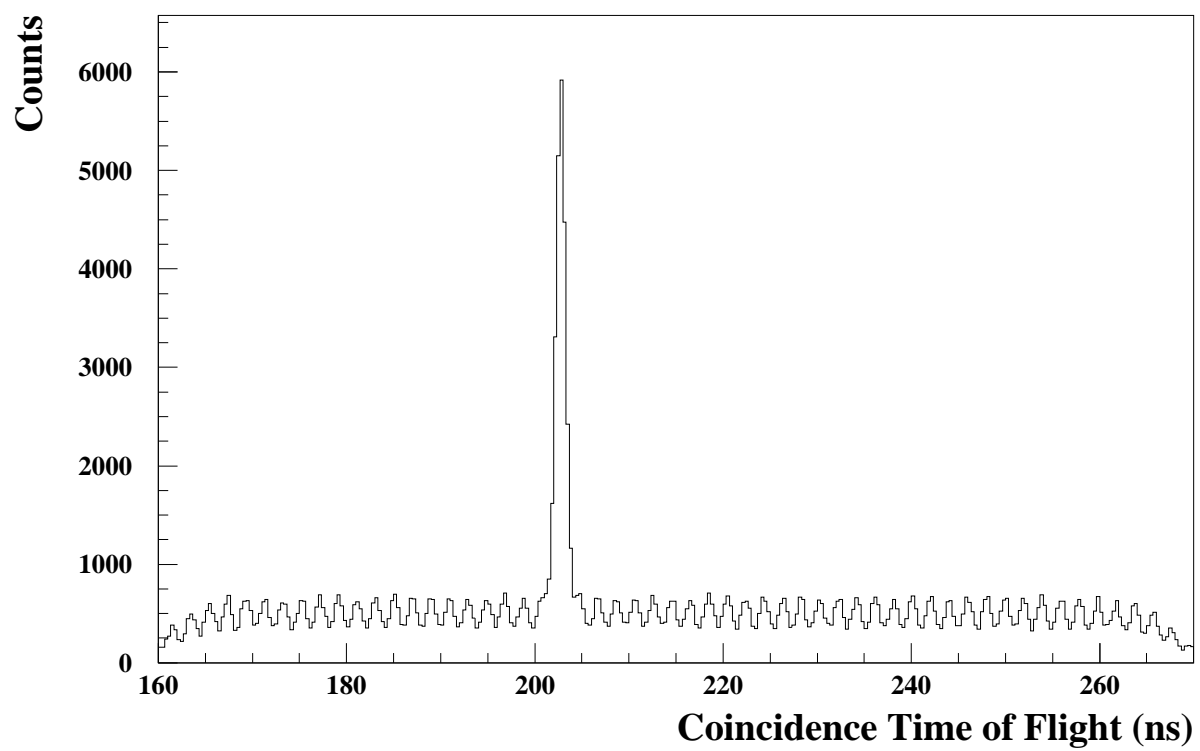

Figure 3-8: Corrected coincidence time-of-flight spectrum after the $y_{t g}$ vertex cut to reduce accidental coincidence events has been applied $\left(\mathrm{E}_{0}=2442.3 \mathrm{MeV}, \theta_{p q}=-16^{\circ}\right.$ kinematic setting).

\subsection{Pion Rejection}

The values of $\omega$ used for this experiment, $445 \mathrm{MeV}$ for the quasielastic kinematics and $589 \mathrm{MeV}$ for the dip region kinematics, are large enough to produce pions. There are 
three main channels through which a single pion can be produced when an electron interacts with a nucleon

$$
\begin{aligned}
& e+p \rightarrow e^{\prime}+p+\pi^{0} \\
& e+p \rightarrow e^{\prime}+n+\pi^{+} \\
& e+n \rightarrow e^{\prime}+p+\pi^{-}
\end{aligned}
$$

The reaction 3.8 with an electron and a proton in the final state gives a true $\left(\mathrm{e}, \mathrm{e}^{\prime} \mathrm{p}\right)$ coincidence. It will be shown in chapter 5 that this reaction makes a large contribution to the $\left(e, e^{\prime} p\right)$ cross section at high missing energies.

However, the reactions 3.9 and 3.10 do not represent true $\left(e, e^{\prime} p\right)$ coincidences. Reaction 3.9 can generate a coincidence between an electron and a $\pi^{+}$detected in the hadron spectrometer instead of a proton, while reaction 3.10 can generate a coincidence between a proton and a $\pi^{-}$detected in the electron spectrometer. In order to calculate the electron-proton coincidence cross section, the contributions from these two reactions have to be removed.

Throughout this experiment the hadron spectrometer momentum was around 1 $\mathrm{GeV} / \mathrm{c}$. At this momentum the velocity of a $\pi^{+}$traveling through the spectrometer is $\sim 1.0 \mathrm{c}$ while the velocity of a proton is $\sim 0.7 \mathrm{c}$. Since the path length through the spectrometer is about $27 \mathrm{~m}$, the difference between the velocities of the two particles translates to a flight time difference of $\sim 30 \mathrm{~ns}$. As a result, the coincidence timeof-flight for a $\left(e, e^{\prime} \pi^{+}\right)$event would be shorter by about $30 \mathrm{~ns}$ than the time-of-flight for a $\left(e, e^{\prime} p\right)$ event. Therefore, with the real coincidence TOF window selected $\pm 8 \mathrm{~ns}$ around the true (e,e $\left.e^{\prime} p\right)$ peak, the coincidences from $\left(e, e^{\prime} \pi^{+}\right)$are easily excluded.

Since the velocity of $\pi^{-}$traveling through the electron spectrometer was very close to the velocity of the electrons, the available coincidence TOF resolution did not permit the separation of $\left(\mathrm{e}, \mathrm{e}^{\prime} \mathrm{p}\right)$ and $\left(e, \pi^{-} p\right)$ peaks using the coincidence TOF spectrum. Instead the gas Cherenkov detector on the electron spectrometer was used to reject pions. Fig. 3-9 shows typical TDC and ADC spectra from the Cherenkov detector. In the ADC spectrum shown, the pedestal is around the $450^{\text {th }}$ channel, while the single photo-electron peak is around the $520^{\text {th }}$ channel. A cut was placed 
to reject all the events below half of the single photo-electron peak.
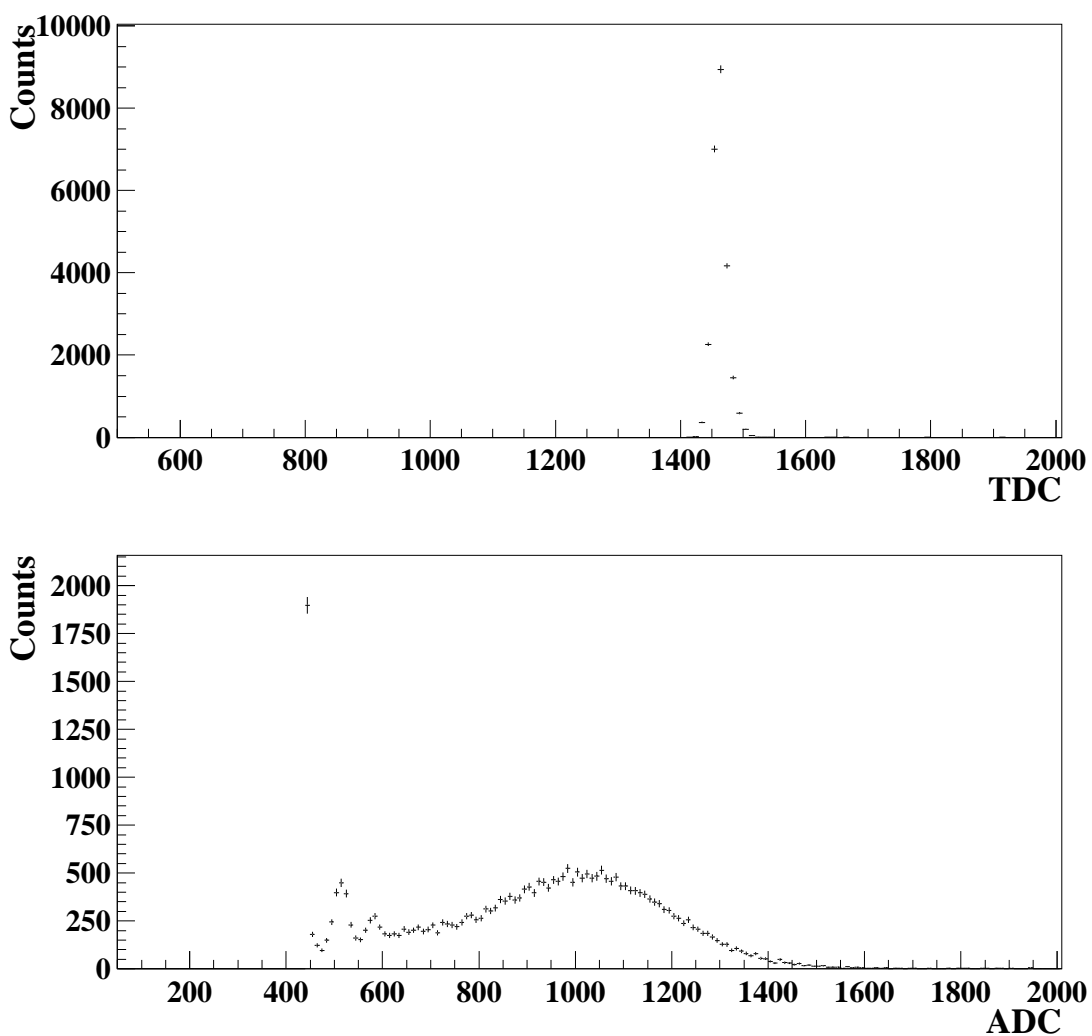

Figure 3-9: Typical TDC (top) and a ADC (bottom) spectra from the gas Cherenkov detector

In the dip region, the cross section was measured up to a very high missing energy of $320 \mathrm{MeV}$. At high missing energies, the (e,e'p) data are more susceptible to contamination due to $\left(\mathrm{e}, \mathrm{e} \pi^{-} \mathrm{p}\right)$ events.

The reaction 3.10 can proceed through the mediation of a virtual photon or a real photon (water foils acting as radiators of real photons)

$$
\begin{gathered}
e+n \rightarrow e^{\prime}+\gamma^{\star}+n \rightarrow e^{\prime}+\pi^{-}+p \\
\gamma+n \rightarrow \pi^{-}+p .
\end{gathered}
$$


Energy conservation for these two processes gives (neglecting target recoil)

$$
E_{0}+M_{A}=E_{e^{\prime}}+E_{\pi}+M_{A-1}+E_{p}
$$

where $M_{A}$ is the mass of the target nucleus and $M_{A-1}$ is the mass of the residual nucleus. Using $E_{p}=T_{p}+M_{p}$ and $M_{A}-M_{A-1}=M_{n}-E_{B}$ (where $T_{p}$ is the kinetic energy of the final proton, $E_{B}$ is the binding energy of the neutron and $M_{p}$ and $M_{n}$ are the masses of the proton and the neutron respectively) eqn. 3.13 can be rewritten as

$$
E_{0}-T_{p}=E_{B}-M_{n}+M_{p}+E_{\pi}+E_{e^{\prime}}
$$

Even if the $\pi^{-}$is detected by the electron spectrometer, the missing energy for the event is calculated by assuming that the detected particle is an ultra-relativistic electron; that is, $E_{H R S E}=P_{H R S E}$, where $E_{H R S E}$ and $P_{H R S E}$ are the energy and momentum measured on the electron spectrometer.

$$
E_{m i s s}=E_{0}-P_{H R S E}-T_{p}=E_{0}-P_{\pi}-T_{p}
$$

Substituting for $E_{0}-T_{p}$ from eqn. 3.14 and neglecting the mass difference between a proton and a neutron yields

$$
E_{\text {miss }}=E_{B}+E_{\pi}-P_{\pi}+E_{e^{\prime}}
$$

For a $\pi^{-}$detected by the electron spectrometer at its central momentum for the dip region kinematic setting $\left(P_{\pi}=1056 \mathrm{MeV} / \mathrm{c}\right), E_{\pi} \cong 1065 \mathrm{MeV}$. For a neutron ejected from a p shell $E_{B} \cong 15 \mathrm{MeV}$. Substituting these values in eqn. 3.16 yields

$$
E_{m i s s} \cong 24 \mathrm{MeV}+E_{e^{\prime}}
$$

Thus, the $E_{\text {miss }}$ threshold for processes given in eqns. 3.11 and 3.12 is around 24 $\mathrm{MeV}$. The available phase-space for these processes grows with increasing $E_{e^{\prime}}$ and hence with increasing $E_{m i s s}$. 

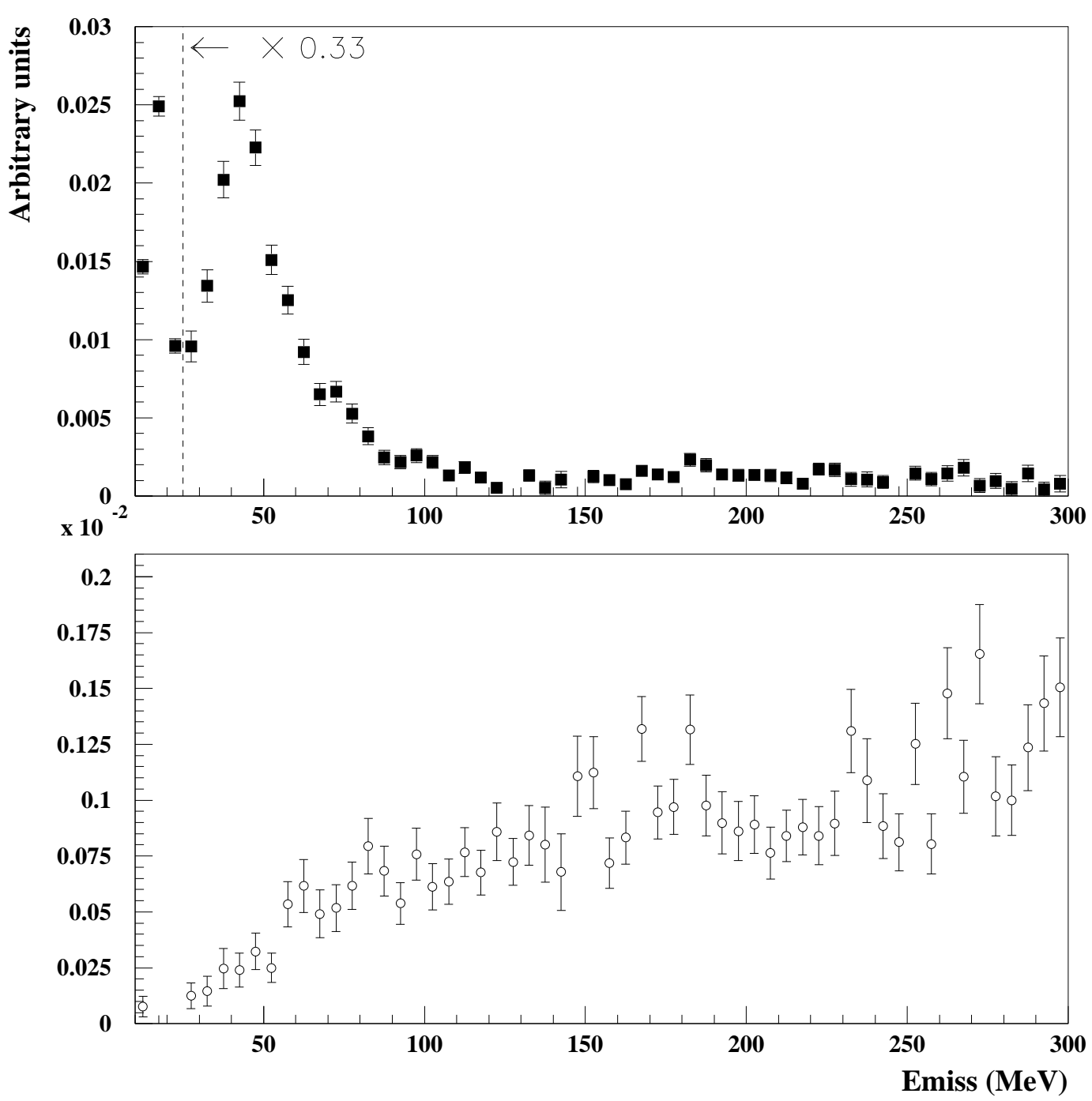

Figure 3-10: Events accepted by the Cherenkov cut (top plot) compared to to events rejected for the dip region data. Note the factor of $10^{-2}$ on the vertical scale of the rejected plot.

Fig. 3-10 shows the yield for the distribution of events rejected by the Cherenkov cut compared to that for the events accepted for the dip region data (Note the factor of $10^{-2}$ on the vertical scale of the rejected plot). The absence of rejected yield for $E_{\text {miss }} \lesssim 25 \mathrm{MeV}$ indicates the high electron detection efficiency of the Gas Cherenkov detector. To quantify the electron detection efficiency, the ratio between the rejected 
and the accepted strengths for $E_{\text {miss }}<24 \mathrm{MeV}$ was calculated:

$$
R=\frac{\int_{E_{m i s s}=0 \mathrm{MeV}}^{24 \mathrm{MeV}}(\text { Events rejected by the Cherenkov cut })}{\int_{E_{\text {miss }}=0 \mathrm{MeV}}^{24 \mathrm{MeV}}(\text { Events accepted by the Cherenkov cut })} .
$$

For the present case, $R=6 \times 10^{-4}$, yielding an electron detection efficiency of $99.94 \%$ for the Cherenkov detector.

At the highest $E_{\text {miss }}$, the rejected strength is comparable to the accepted strength.

\subsection{Events with collimator punch-through protons}

Another source of contamination of the data were the protons that penetrated through the HRSH collimator. The collimator used for this experiment was made out of an 8cm thick plate of lead, with 1-cm wide tungsten "lips" of the same thickness attached to the four sides of the collimator hole. For this experiment, the energy loss suffered by the highest momentum protons passing through the lead in the collimator was approximately $150 \mathrm{MeV}$, while the energy loss due to passing through tungsten was about $230 \mathrm{MeV}$. As a result, the protons from $\mathrm{H}$ and from valence states of ${ }^{16} \mathrm{O}$ can penetrate through the collimator and end up in the deep $E_{\text {miss }}$ continuum of $150 \mathrm{MeV}$ $<E_{\text {miss }}$.

However, only a fraction of the protons that are incident upon the collimator end up at the focal plane. The thicknesses of lead and tungsten in the collimator amount to 0.5 and 1.0 nuclear interaction lengths respectively. This means that about $40 \%$ of the protons traveling through the lead are absorbed, while about $65 \%$ of those traveling through the tungsten are absorbed. The multiple scattering suffered by the protons which penetrate through the collimator has a FWHM angular distribution of approximately $100 \mathrm{mr}$. The solid angle acceptance limits of the spectrometer, mainly set by the Q1 acceptance, are about $\pm 30 \mathrm{mr}$ in-plane and about $\pm 60 \mathrm{mr}$ out-of-plane. Thus, $\sim 80 \%$ of the protons which are incident on the collimator walls cannot reach the focal plane. 

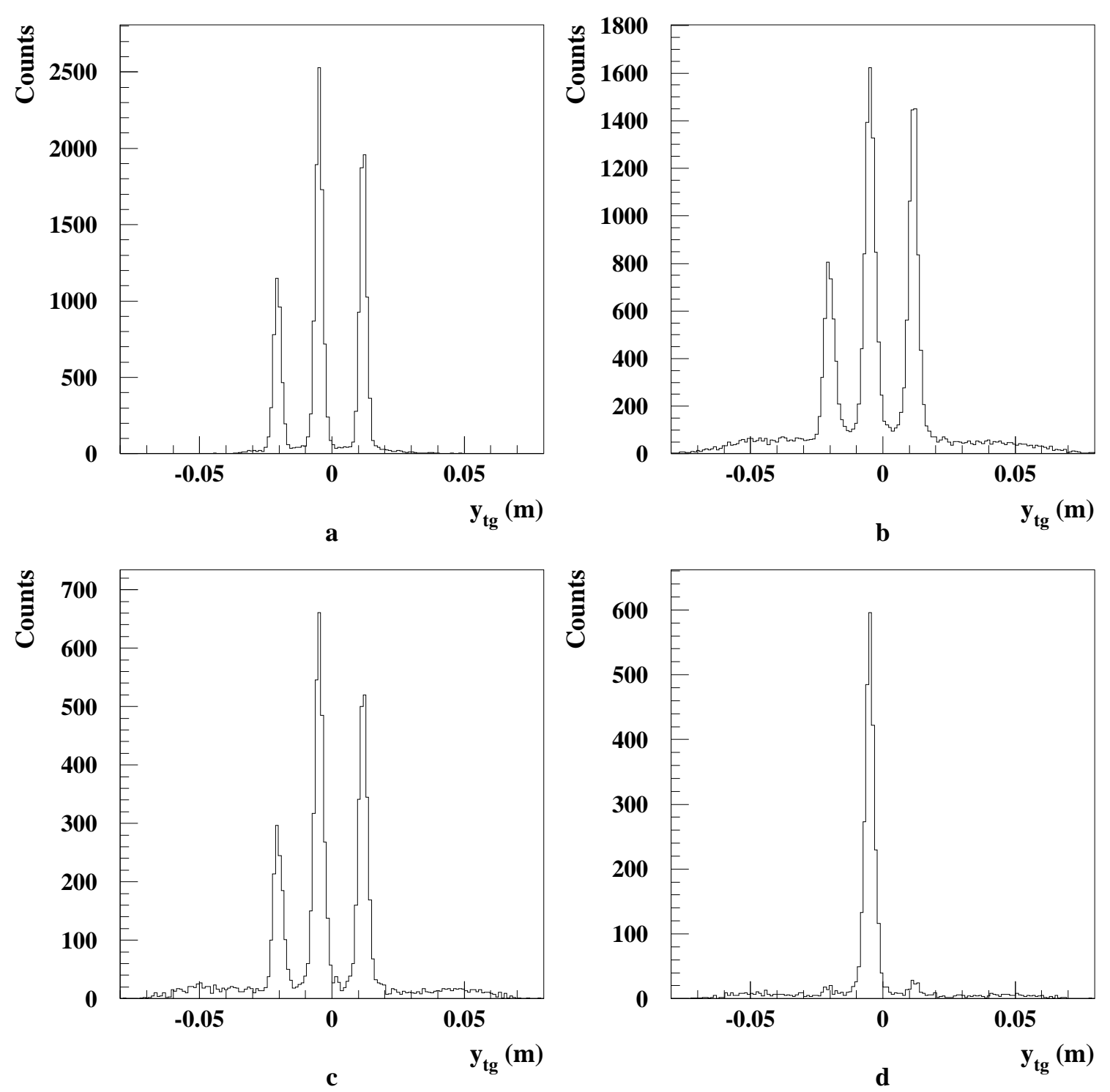

Figure 3-11: HRSH $y_{t g}$ distributions for the dip region kinematics. (a) is for low $E_{m i s s}$ values from $10 \mathrm{MeV}$ to $50 \mathrm{MeV}$, with no solid angle cuts. (b) is for high $E_{\text {miss }}$ values from $150 \mathrm{MeV}$ to 250 $\mathrm{MeV}$ with no solid angle cuts. (c) is for the same $E_{\text {miss }}$ as (b) but with the solid angle cuts used for the experiment (see table 3.3). (d) has the HRSE $y_{t g}$ cut shown in Fig. 3-12 in addition to the solid angle cuts. Note the different vertical scales.

A comparison of the HRSH $y_{t g}$ distribution for a low $E_{m i s s}$ range (Fig. 3-11.a) to a $y_{t g}$ distribution for a moderate $\left(150 \mathrm{MeV}<E_{\text {miss }}<250 \mathrm{MeV}\right) E_{\text {miss }}$ range (Fig. 3-11.b) shows that the higher $E_{\text {miss }} y_{t g}$ distribution has much stronger side tails than the lower $E_{m i s s} y_{t g}$ distribution. This indicates that there is a significant punch- 
through presence at high $E_{m i s s}$ values. The software cuts ${ }^{1}$ employed in the analysis of these data successfully remove most of these events. Fig. 3-11.b was obtained without any solid angle cuts. Fig 3-11.c shows the $y_{t g}$ distribution after the solid angle cuts (see table 3.3) are applied.

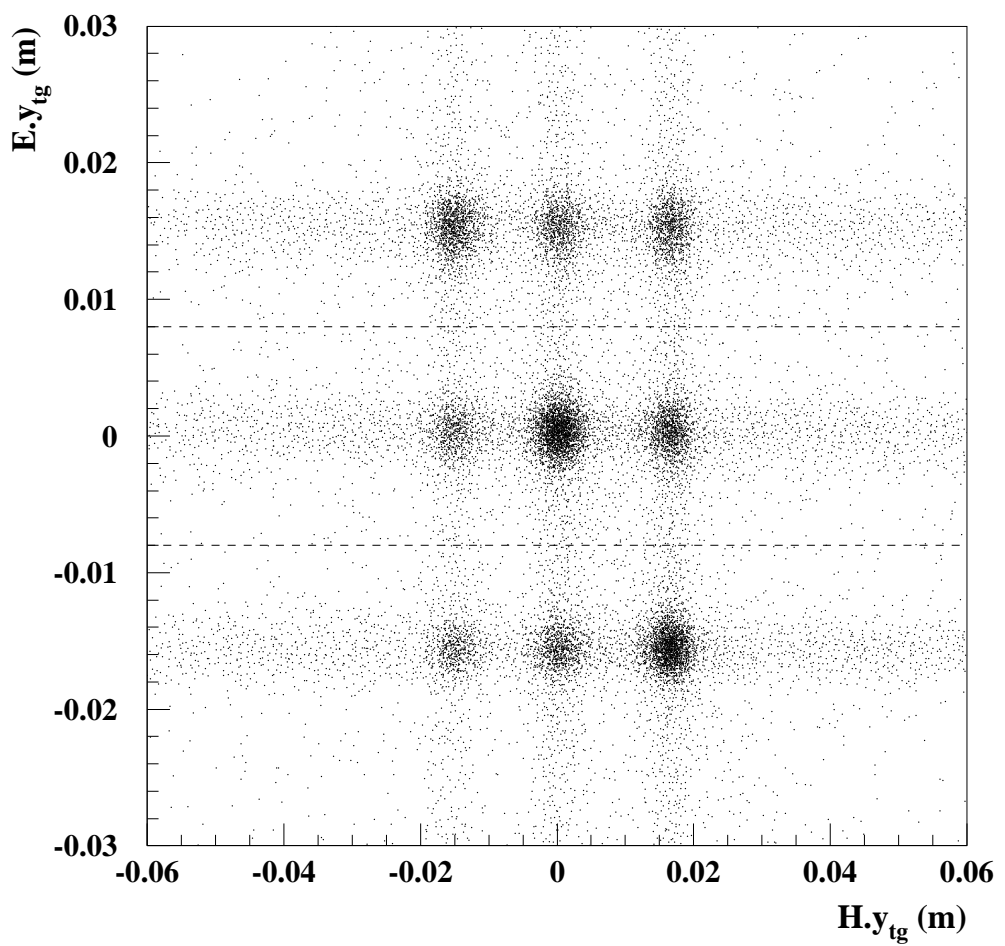

Figure 3-12: HRSE $y_{t g}$ vs. HRSH $y_{t g}$ distribution for the dip region kinematics showing the HRSE $y_{t g}$ vertex cut. The three thick blobs of events on the diagonal correspond mainly to real $\left(e, e^{\prime} p\right)$ events. The other clusters of events are due to accidental coincidences with the electron and the proton from two different foils. Most of the events in the thick horizontal bands extending out from the real clusters correspond to punch-through protons. A HRSE $y_{t g}$ cut as shown in this figure ensures that the punch-throughs from the neighboring foils do not come under the peak of a given foil.

The punch-through background under any one of the peaks shown in Fig. 3-11.b can come from any of the three foils. However, since there is no collimator penetration on the electron side, $y_{t g}$ reconstruction by the HRSE can be used to remove the punchthrough contributions to a given foil from the other two foils. A HRSE $y_{t g}$ cut, as illustrated in Fig. 3-12, results in the HRSH $y_{t g}$ distribution shown in Fig. 3-11.d.

\footnotetext{
${ }^{1}$ The software cuts are given in table 3.3 .
} 


\subsubsection{Estimation of punch-through contamination}

Since Fig. 3-11.d has been obtained with the same cuts employed for the cross section calculation, the tails of this $y_{t g}$ spectrum to either side of the central peak show the level of punch-through contamination of the cross section. In the worst case, this contamination is only about $10 \%$ of the cross section. The following procedure was then employed to remove the punch-through contribution from the data.

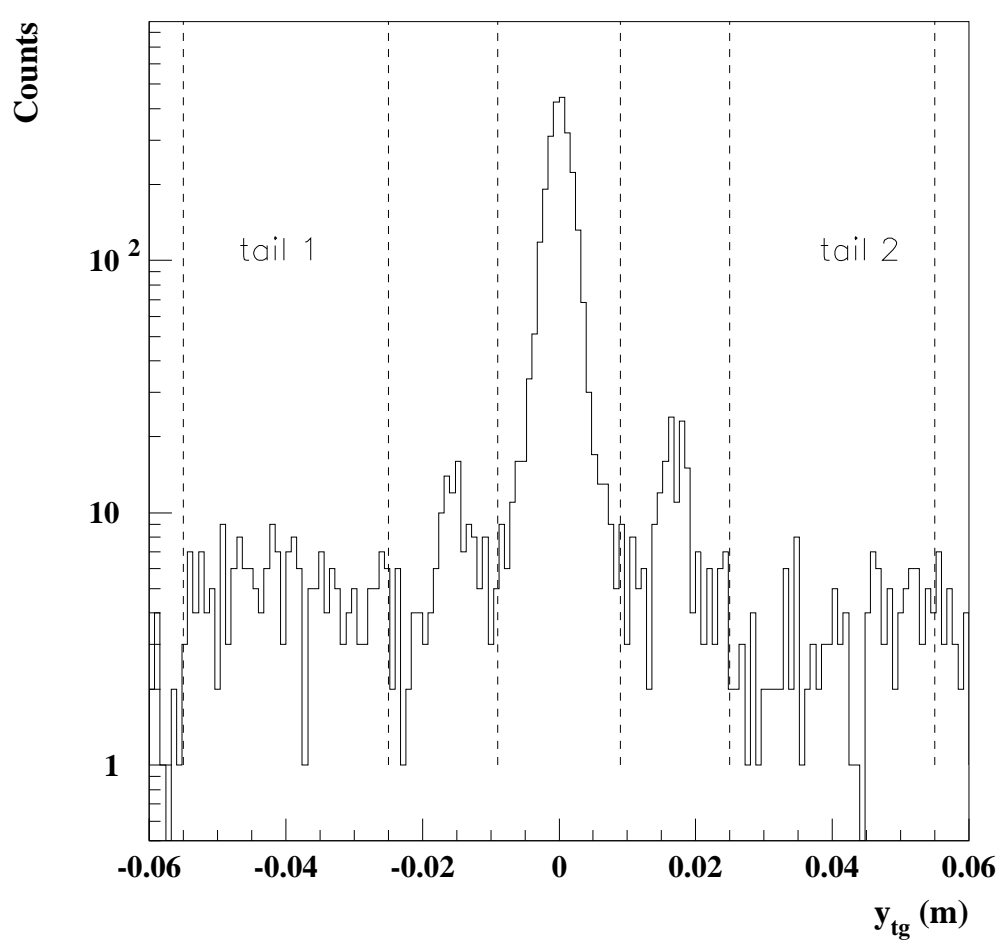

Figure 3-13: HRSH $y_{t g}$ distribution for the dip region kinematics showing the "tail" regions used for the punch-through subtraction. Note the logarithmic scale. The small peaks on either side of the central peak are due to accidental coincidence events with the two members of a coincidence events originating at different water-foils. The coincidence time-of-flight spectra corresponding to these small peaks are flat.

- Two cuts on the flat region of the $y_{t g}$ tail on either side of the central peak (see Fig. 3-13) were made. The "tail" cross section was calculated for the events in these regions. In Fig. 3-14 the cross sections calculated for the tails is compared to the continuum strength for the accepted events. These spectra exhibit several important features: 

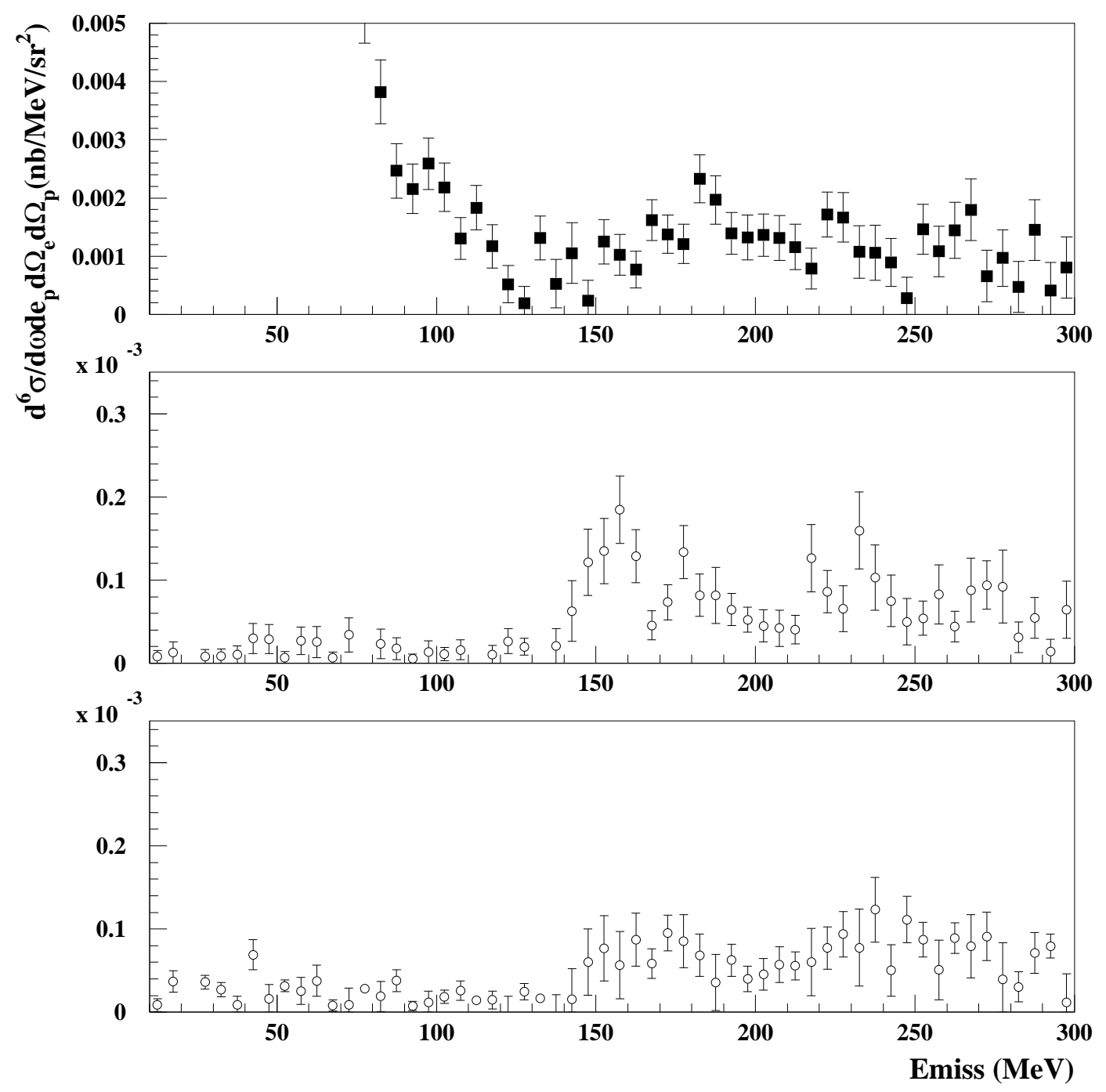

Figure 3-14: The cross sections from the HRSH $y_{t g}$ tails compared to the cross section for the events in the HRSH $y_{t g}$ peak for the dip region kinematics. (a) gives the cross section for events in the $y_{t g}$ peak. while (b) and (c) show cross sections calculated for the tail regions 1 and 2 (defined in Fig. 3-13) respectively. Note the factor of $10^{-3}$ used for the y scale of the "tail" cross sections.

- the spectra from the tails on the two sides of the central peak have the same shape (within the statistical error). This indicates that the missing energy distribution for the punch-throughs should be the same for all $y_{t g}$ values, and justifies the use of the strength in the $y_{t g}$ tails to correct the contamination in the peak region.

- in the worst case, the punch-through contamination is only about $10 \%$ of 
the accepted strength.

- both "tail" region cross section spectra exhibit two bumps, one at 160 MeV and the other one at $\sim 240 \mathrm{MeV}$, at the expected locations (see section 3.5)

- there is very little strength in the tails up to $150 \mathrm{MeV}$. This indicates that the valence state data has negligible punch-through contamination.

- The data from the $y_{t g}$-tails were binned in the same manner as the accepted data.

- The punch-through events in each bin were subtracted using a method similar to the accidental coincidence subtraction. The corrected strength in a given bin was calculated as

$$
N_{\text {corrected }}=N_{\text {peak }}-\frac{W_{\text {peak }}}{W_{\text {tail }}} N_{\text {tail }}
$$

where $N_{\text {peak }}$ is the number of events in the $y_{t g}$ peak, $N_{t a i l}$ is the number of events in the $y_{t g}$ tail sections, $W_{\text {peak }}$ is the width of the peak, and $W_{\text {tail }}$ is the total width of the tail sections.

The results presented in this thesis were obtained after the punch-through subtraction. 


\subsection{Spectrometer normalization}

A response function separation experiment demands precise normalization of cross sections measured at different kinematic settings. This was particularly crucial for the present experiment because the thickness of the waterfall target could change during the experiment, and a continuous thickness-measuring device for the waterfoils was not available. Instead, the $\mathrm{H}\left(\mathrm{e}, \mathrm{e}^{\prime}\right)$ elastic cross section measured by the electron spectrometer was used as a continuous luminosity monitor, providing the desired precise normalization for the various kinematic settings.

Each cross section measured in this experiment could be normalized to the $\mathrm{H}\left(\mathrm{e}, \mathrm{e}^{\prime}\right)$ cross section because $\mathrm{H}\left(\mathrm{e}, \mathrm{e}^{\prime}\right)$ elastic events were scattered into the center of the HRSE focal plane for all kinematic settings. Since this was the first experiment performed in Hall A, the absolute cross section for $\mathrm{H}\left(\mathrm{e}, \mathrm{e}^{\prime}\right)$ for each setting was also calculated. This was compared to the $\mathrm{H}\left(\mathrm{e}, \mathrm{e}^{\prime}\right)$ "world" cross section used for normalization.

\subsubsection{Waterfall target thickness measurement}

The waterfall target thickness was calculated by comparing the ${ }^{16} \mathrm{O}\left(\mathrm{e}, \mathrm{e}^{\prime}\right)$ elastic cross section measured for the waterfall target to the same cross section measured for a thin $\mathrm{BeO}$ target of known thickness. The BeO target thickness of $155 \mathrm{mg} \mathrm{cm} \mathrm{cm}^{-2}$ was measured to $1.5 \%$ accuracy using a high precision electronic balance and an electronic vernier caliper. At the beginning of the experiment, ${ }^{16} \mathrm{O}\left(\mathrm{e}, \mathrm{e}^{\prime}\right)$ elastic data were acquired with the $\mathrm{BeO}$ target. Immediately after that ${ }^{16} \mathrm{O}\left(\mathrm{e}, \mathrm{e}^{\prime}\right)$ elastic data were collected from the waterfall target under similar conditions. The number of events under the elastic peak was determined for both spectra by integrating over a $4.0 \mathrm{MeV}$ interval in $\omega$. Only the central water foil was used for this comparison because the $y_{t g}$ position of the central foil was similar to the $y_{t g}$ position of the BeO target. This ensured that the spectrometer acceptance was the same for both targets. 


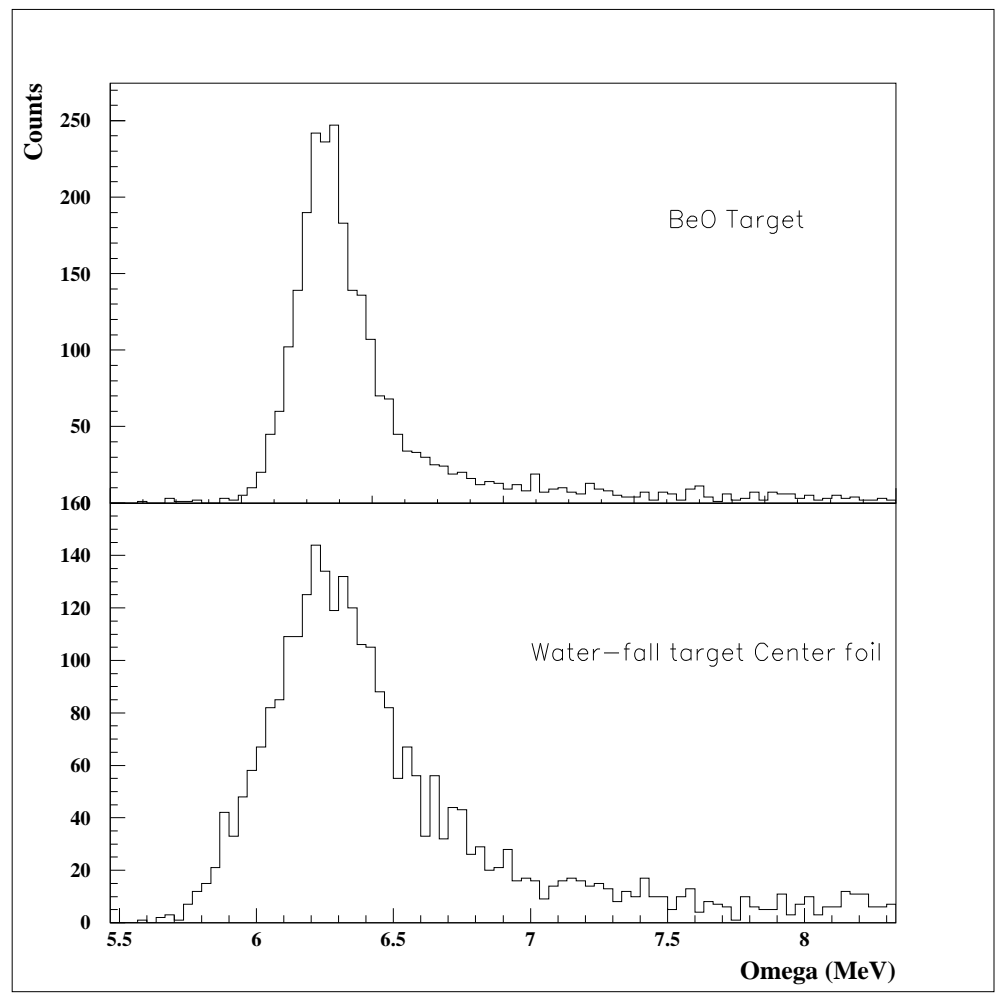

Figure 3-15: ${ }^{16} \mathrm{O}\left(\mathrm{e}, \mathrm{e}^{\prime}\right)$ elastic peak obtained for $\mathrm{BeO}$ and $\mathrm{H}_{2} \mathrm{O}$ targets. Note that the peak is widened for the waterfall target due to the multiple scattering suffered by the electrons going through the waterfall chamber windows.

The $\mathrm{BeO}$ target thickness was similar to the thickness of a single water-foil. However due to the presence of three water-foils and the waterfall chamber windows, the path length of the electrons through heavy material is much longer for the waterfall target than that for the $\mathrm{BeO}$ target. As a result, the probability of external bremsstrahlung radiation is higher for the central water foil than for the $\mathrm{BeO}$ target, as apparent in Fig. 3-15. External bremsstrahlung correction factors were calculated separately and were applied to the yield from each target to account for this difference. The internal (Schwinger) radiative correction is same for both targets and hence drops out of the calculation.

The charge measured by the beam cavity monitors and recorded by the $\mathrm{V}$ to $\mathrm{F}$ scaler was used to normalize the two runs. Since it involved the comparison of two runs taken next to each other, this measurement is not sensitive to the absolute normalization of the beam cavity monitors. 


\begin{tabular}{|c|c|}
\hline Foil number & thickness $\left(\mathrm{mg} \mathrm{cm}^{-2}\right)$ \\
\hline 1 & $110.0 \pm 4.0$ \\
\hline 2 & $113.0 \pm 3.8$ \\
\hline 3 & $111.0 \pm 4.0$ \\
\hline
\end{tabular}

Table 3.2: Calculated water foil thicknesses. Foil number increases in the direction of the beam.

Once the thickness of the center water foil was calculated using the above method, the thickness of each side foil was calculated by comparing the $\mathrm{H}\left(\mathrm{e}, \mathrm{e}^{\prime}\right)$ elastic cross section from the middle foil to that from the side foil. Again, the external bremsstrahlung correction factors were applied separately to account for the difference of path-lengths through heavy material.

Since the above methods involve only comparisons of cross sections measured under similar conditions, systematic errors are smaller than $1 \%$. The errors given in table 3.2 are dominated by statistics.

\subsection{2 $\mathrm{H}\left(\mathrm{e}, \mathrm{e}^{\prime}\right)$ cross section calculation}

The $\mathrm{H}\left(\mathrm{e}, \mathrm{e}^{\prime}\right)$ elastic cross section can be calculated from experimental data using the equation

$$
\left(\frac{d \sigma}{d \Omega}\right)=\frac{e A}{N_{A} \rho t C \Omega_{e} \varepsilon} N R D,
$$

where $e$ is the electron charge,

$A$ is the atomic weight of $\mathrm{H}$, $N_{A}$ is the Avagadro's number, $\rho t$ is the target thickness,

$C$ is the accumulated charge, $\Omega_{e}$ is the solid angle, $\varepsilon$ is the trigger efficiency, $D$ is the computer dead time correction,

$N$ is the number of events in the $\mathrm{H}\left(\mathrm{e}, \mathrm{e}^{\prime}\right)$ elastic peak integrated over an energy interval $\Delta E$, and

$R$ is the radiative correction for the events radiated out of the interval $\Delta E$. 
$\mathrm{H}\left(\mathrm{e}, \mathrm{e}^{\prime}\right)$ data were acquired simultaneously with ${ }^{16} \mathrm{O}\left(\mathrm{e}, \mathrm{e}^{\prime} \mathrm{p}\right)$ data for every kinematic setting of the experiment. Special care was taken to set the electron singles pre-scale factor low enough to gather enough $\mathrm{H}\left(\mathrm{e}, \mathrm{e}^{\prime}\right)$ events for a one percent cross section measurement for each setting. These $\mathrm{H}\left(\mathrm{e}, \mathrm{e}^{\prime}\right)$ events were used to normalize the measured ${ }^{16} \mathrm{O}\left(\mathrm{e}, \mathrm{e}^{\prime} \mathrm{p}\right)$ cross sections. Further the $\mathrm{H}\left(\mathrm{e}, \mathrm{e}^{\prime}\right)$ cross section was calculated using the data from the central water-foil at each setting of the electron spectrometer. This cross section was compared to the $\mathrm{H}\left(\mathrm{e}, \mathrm{e}^{\prime}\right)$ "world" cross section used for normalization to provide a check of the systematics.

The $\mathrm{H}\left(\mathrm{e}, \mathrm{e}^{\prime}\right)$ elastic peak sits on a smooth quasi-elastic ${ }^{16} \mathrm{O}$ bump. The peak fitting code ALLFIT [63] was used to separate the $\mathrm{H}$ elastic peak from the background (Fig. 3-16). ALLFIT parameterizes the background with a polynomial and includes this polynomial in the function to fit the data. We have used a third order polynomial to parameterize the background and included a portion of the ${ }^{16} \mathrm{O}$ quasi-elastic bump below the $\mathrm{H}\left(\mathrm{e}, \mathrm{e}^{\prime}\right)$ elastic peak to determine the behavior of the background.

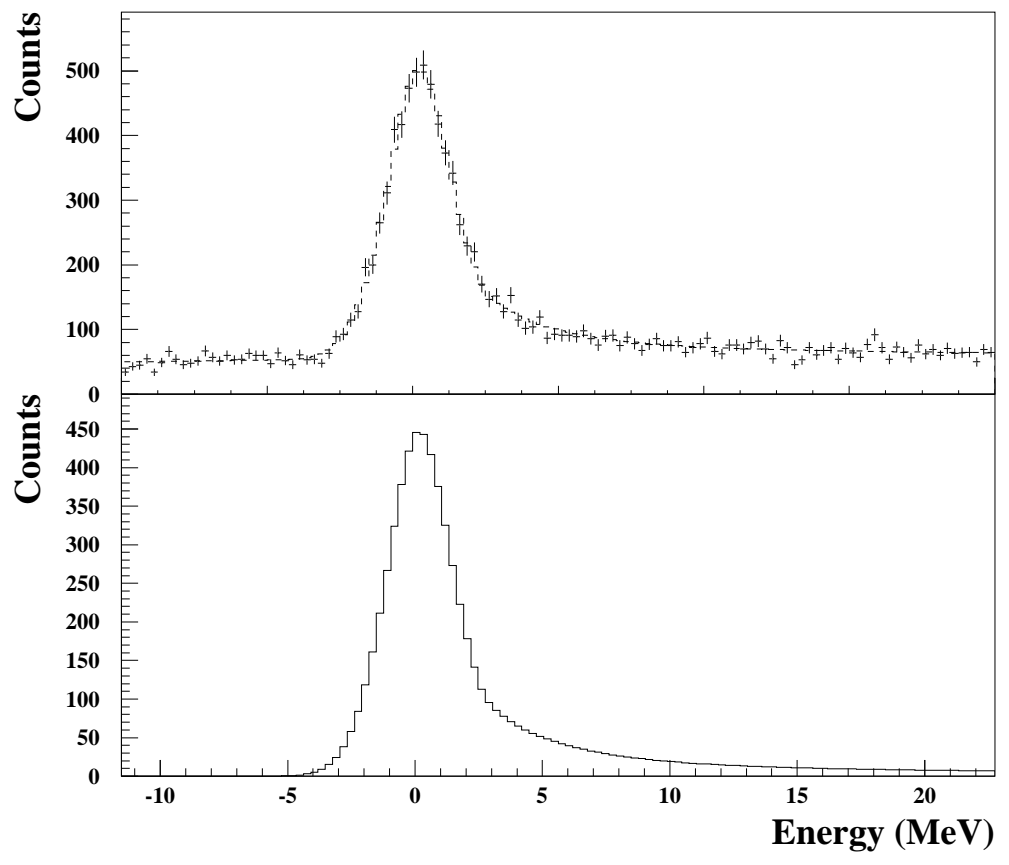

Figure 3-16: The top plot shows the $\mathrm{H}\left(\mathrm{e}, \mathrm{e}^{\prime}\right)$ elastic peak sitting on the ${ }^{16} \mathrm{O}$ quasi-elastic peak with the ALLFIT fit superimposed. The lower plot shows the elastic peak with background subtracted by ALLFIT.

After subtracting the background, ALLFIT applies radiative corrections to the 
integrated yield. To calculate the $\mathrm{H}\left(\mathrm{e}, \mathrm{e}^{\prime}\right)$ cross section for the central foil, the target thickness calculated using the "BeO method" was used. Accumulated charge was obtained from the calibrated $\mathrm{V}$ to $\mathrm{F}$ scaler data from beam current monitors. The data acquisition dead time correction factor for the singles events was determined by the ratio $\frac{S 1}{T 1}$ where $S 1$ is the total number of electron singles events counted by the scaler module and $T 1$ is the total number of electron singles events written to tape.

\subsubsection{Normalization of $\mathbf{H}\left(\mathbf{e}, \mathbf{e}^{\prime}\right)$ cross section to world data}

The measured $\mathrm{H}\left(\mathrm{e}, \mathrm{e}^{\prime}\right)$ cross sections were compared to the available world cross section data. In order to obtain world $\mathrm{H}\left(\mathrm{e}, \mathrm{e}^{\prime}\right)$ cross sections at the $Q^{2}$ value for this experiment, the "Mainz Form Factor Parameterization" [64] was used. This parameterization has been obtained by fitting to Mainz $\mathrm{H}\left(\mathrm{e}, \mathrm{e}^{\prime}\right)$ data up to $Q^{2}=0.7 \mathrm{GeV}^{2}$. $\mathrm{H}\left(\mathrm{e}, \mathrm{e}^{\prime}\right)$ cross section values obtained through the Mainz parameterization have been compared to the $\mathrm{H}$ elastic cross sections measured at MIT-Bates previously, and have shown to be in very good agreement [65].

The $\mathrm{H}\left(\mathrm{e}, \mathrm{e}^{\prime}\right)$ elastic cross section can be written as

$$
\frac{d \sigma}{d \Omega}=\frac{\sigma_{M}}{\eta}|F|^{2}
$$

where the form factor $F$ can be written in terms of Sachs form factors

$$
|F|^{2}=\frac{G_{E}^{2}+\tau G_{M}^{2}}{1+\tau}+2 \tau G_{M}^{2} \tan ^{2}\left(\theta_{\mathrm{e}} / 2\right)
$$

the recoil factor $\eta$ is given by

$$
\eta=1+\frac{2 E_{0}}{M_{p}} \sin ^{2}\left(\theta_{\mathrm{e}} / 2\right)
$$

and $\tau$ is given by

$$
\tau=\frac{Q^{2}}{4 M_{p}^{2}}
$$

The Mott cross section $\sigma_{M}$ is the electron scattering cross section for an infinitely 
heavy unit positive charge and can be written as

$$
\sigma_{M}=\frac{\alpha^{2} \cos ^{2}\left(\theta_{\mathrm{e}} / 2\right)}{4 E_{0}^{2} \sin ^{2}\left(\theta_{\mathrm{e}} / 2\right)}
$$

The Mainz electric and magnetic proton form factor parameterizations are given by

$$
G_{E}\left(Q^{2}\right)=\frac{0.312}{1+Q^{2} / 6.0}+\frac{1.312}{1+Q^{2} / 15.02}-\frac{0.709}{1+Q^{2} / 44.08}+\frac{0.085}{1+Q^{2} / 154.2}
$$

and

$$
\frac{G_{M}\left(Q^{2}\right)}{\mu}=\frac{0.694}{1+Q^{2} / 8.5}-\frac{0.719}{1+Q^{2} / 15.02}+\frac{0.418}{1+Q^{2} / 44.08}+\frac{0.005}{1+Q^{2} / 355.4}
$$

where $\mu$ is the proton magnetic dipole moment.

The normalized cross section results for the perpendicular kinematics settings at all three beam energies are shown in Fig. 3-17. The uncertainty in the cross section is dominated by the uncertainty in the waterfall target thickness. For all three beam energies, the measured cross sections are in good agreement with the Mainz values, indicating that the systematics of the cross section measurement during this experiment were under control.

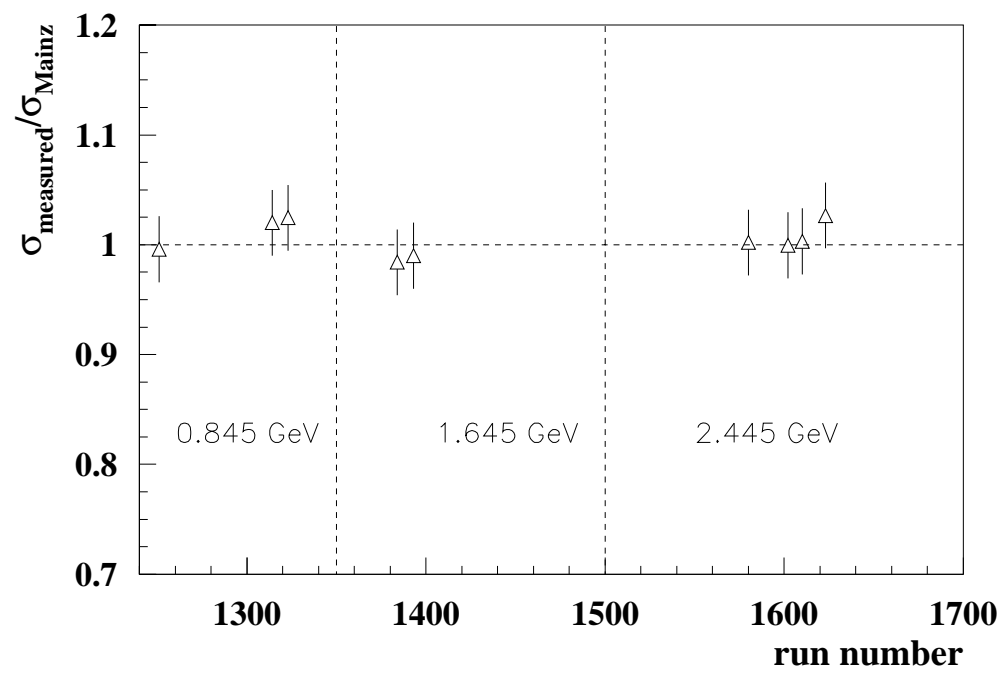

Figure 3-17: Measured $\mathrm{H}\left(\mathrm{e}, \mathrm{e}^{\prime}\right)$ elastic cross section normalized to the cross sections predicted by Mainz parameterization. The results are presented here for each perpendicular kinematics setting with the run number indicating the first run in the group. 


\subsubsection{Hadron spectrometer trigger efficiency determination using $\mathbf{H}\left(\mathbf{e}, \mathrm{e}^{\prime} \mathbf{p}\right)$}

The overall efficiency for the $\left(e, e^{\prime} p\right)$ reaction is given by the product of the electron spectrometer efficiency $\left(\epsilon_{e}\right)$, the hadron spectrometer efficiency $\left(\epsilon_{p}\right)$ and the coincidence trigger efficiency $\left(\epsilon_{\text {coin }}\right)$ :

$$
\epsilon=\epsilon_{e} \epsilon_{p} \epsilon_{c o i n}
$$

After determining the electron spectrometer efficiency, we used the $H\left(e, e^{\prime} p\right)$ reaction to determine $\epsilon_{p} \epsilon_{\text {coin }}$. In parallel kinematics the two spectrometers are set at the correct angles and the magnetic fields to detect electrons and protons from the $\mathrm{H}\left(\mathrm{e}, \mathrm{e}^{\prime} \mathrm{p}\right)$ reaction. In this setting, when an electron scatters off a proton and is detected in the electron spectrometer, the corresponding proton should be detected in the hadron spectrometer given that the hadron spectrometer acceptance is large enough. An electron detected without the corresponding proton would indicate an inefficiency of either the hadron spectrometer or the coincidence trigger.

The solid angle acceptance of the electron spectrometer combined with two body $\mathrm{H}\left(\mathrm{e}, \mathrm{e}^{\prime} \mathrm{p}\right)$ kinematics yields the angular spread of the corresponding protons. In order to perform a meaningful efficiency check, one has to make sure that the hadron spectrometer angular acceptance is large enough to include the distribution of the protons completely.

This was the case for the $843.3 \mathrm{MeV}$ parallel kinematics setting of the present experiment. For this setting, the electron spectrometer central momentum was 400 $\mathrm{MeV}$ while $|\vec{q}|$ (and the proton spectrometer central momentum) was $\sim 1 \mathrm{GeV}$. Thus, the angular spread of the the protons was 2.5 times smaller than the angular spread of electrons in both the in-plane and out-of-plane angles. Since the solid angle acceptance is the same for the two spectrometers, all the $H\left(e, e^{\prime} p\right)$ protons were detected in the middle region of the hadron acceptance with a sizable margin at each of the four sides. Having wide enough margins in the hadron acceptance ensures that the

protons that multiply scatter away from this angular envelope are still within the hadron arm angular acceptance.

The following procedure was used to calculate $\epsilon_{p} \epsilon_{\text {coin }}$. 
- Data were acquired for the $843.3 \mathrm{MeV}$ parallel kinematics setting with both the electron singles pre-scale factor and the coincidence pre-scale factor set to 1.

- A histogram of electron spectrometer relative momentum (dp/p) cut on coincidence triggers was generated. As shown in Fig. 3-18.a, this histogram shows the $\mathrm{H}\left(\mathrm{e}, \mathrm{e}^{\prime} \mathrm{p}\right)$ elastic peak on a very small ${ }^{16} \mathrm{O}\left(\mathrm{e}, \mathrm{e}^{\prime} \mathrm{p}\right)$ background.

- A histogram similar to the above was generated, but cut on "anti-coincidence events", which are electron singles events without a corresponding proton detected on the hadron spectrometer (Fig. 3-18.b). This spectrum shows a high ${ }^{16} \mathrm{O}\left(\mathrm{e}, \mathrm{e}^{\prime} \mathrm{p}\right)$ background. Any $\mathrm{H}\left(\mathrm{e}, \mathrm{e}^{\prime}\right)$ events present in this spectrum would indicate an inefficiency of the hadron spectrometer.

- In order to find the amount of $\mathrm{H}\left(\mathrm{e}, \mathrm{e}^{\prime}\right)$ elastic events in spectrum 3-18.b, the following procedure was used:

- A polynomial fit to the "non-peak region" of this spectrum $(0.01 \%<|\mathrm{dp} / \mathrm{p}|)$ was used to determine the ${ }^{16} \mathrm{O}\left(\mathrm{e}, \mathrm{e}^{\prime} \mathrm{p}\right)$ background level.

- This background level was then subtracted from spectrum 3-18.b yielding spectrum 3-18.c. As this spectrum indicates, there is only a small number of events at the location of the $\mathrm{H}\left(\mathrm{e}, \mathrm{e}^{\prime}\right)$ elastic peak. This indicates a high level of efficiency for the hadron spectrometer and the coincidence trigger.

- The product $\epsilon_{p} \epsilon_{\text {coin }}$ was quantified by comparing the number of coincidence $\mathrm{H}\left(\mathrm{e}, \mathrm{e}^{\prime}\right)$ events (Fig. 3-18.a) to the number of "anti-coincidence" H(e,e') events (Fig. 3-18.c). This was accomplished by integrating histograms (a) and (c) over a $0.04 \%(\mathrm{dp} / \mathrm{p})$ interval centered on the $\mathrm{H}\left(\mathrm{e}, \mathrm{e}^{\prime}\right)$ elastic peak, and comparing the yields.

This check yields an inefficiency of $1.1 \%$. We believe that the proton absorption in the waterfall target, spectrometer windows and the scintillators can explain this inefficiency. We corrected all measured ${ }^{16} \mathrm{O}\left(\mathrm{e}, \mathrm{e}^{\prime} \mathrm{p}\right)$ cross sections for this inefficiency. 


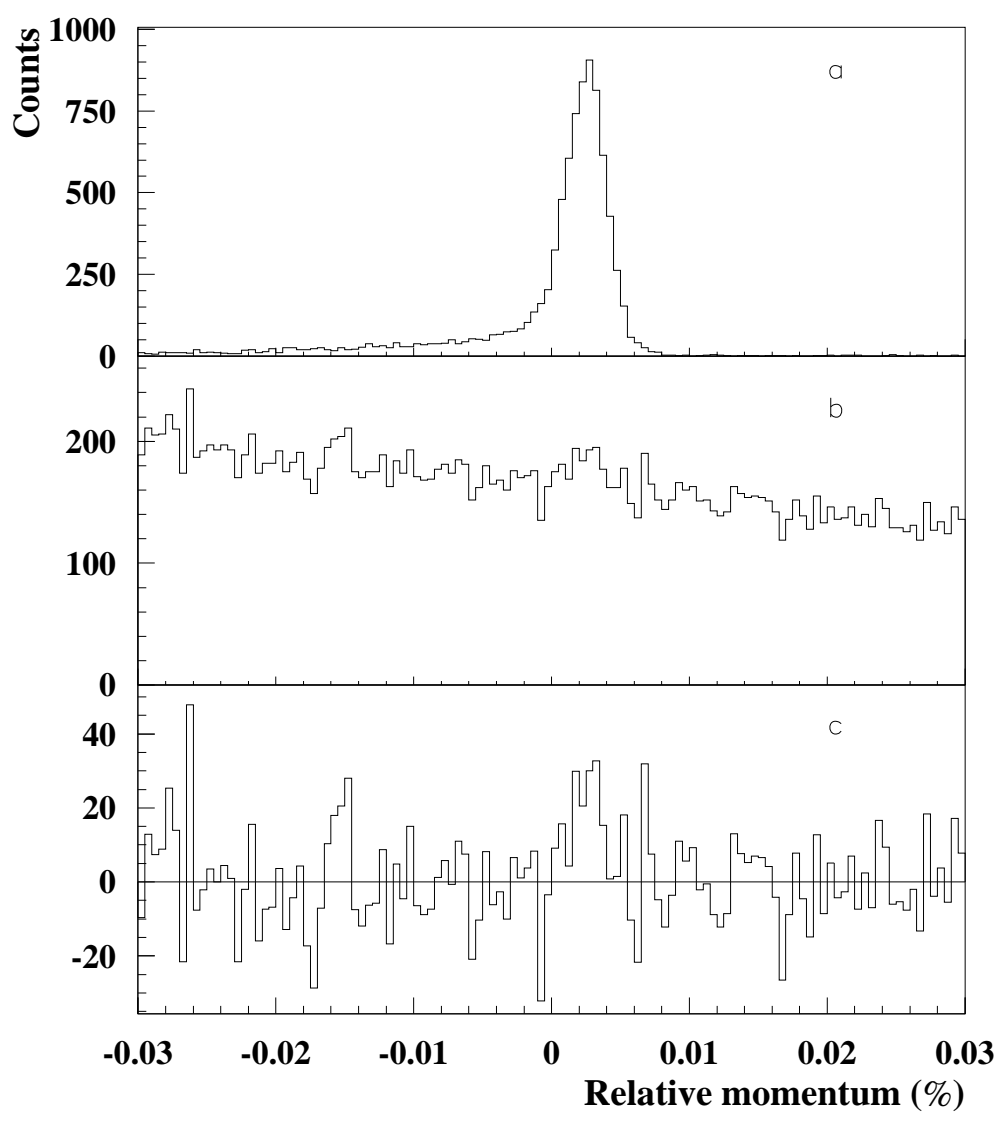

Figure 3-18: The electron spectrometer relative momentum (dp/p) for the $E_{0}=843.3 .0 \mathrm{MeV}$ parallel kinematics setting. Plot (a) is cut on coincidence triggers, and plot (b) on "anti-coincidences" (electron singles events without a corresponding proton detected on the hadron spectrometer). The ${ }^{16} \mathrm{O}\left(\mathrm{e}, \mathrm{e}^{\prime} \mathrm{p}\right)$ quasi-elastic background has been subtracted from (b) to generate plot (c).

\subsubsection{Focal plane relative efficiency}

To determine the relative efficiency profile of the focal plane with respect to the center, the "white spectra" technique was used. Since this procedure has been described in detail in several previous theses [40], [36], [65], only a brief outline is given here.

The basic principal of the white spectra technique is to measure the same cross section at several points along the focal plane. The change in the yield measured at different points reflects the variation of focal plane efficiency between these points. The use of a white spectrum (a smoothly varying distribution) instead of a sharp peak allows one to cover the entire focal plane in several steps.

For the present experiment, ${ }^{16} \mathrm{O}\left(\mathrm{e}, \mathrm{e}^{\prime}\right)$ and ${ }^{16} \mathrm{O}(\mathrm{e}, \mathrm{p})$ spectra from the dip region 
were measured at five points on the HRSE and HRSH respectively. The program RELEFF [40] was used to deconvolute the focal plane efficiency from the measured white spectra. The formalism of the deconvolution is given in [1], and only the results from this analysis are presented here.

Figures 3-19 and 3-20 show the deconvoluted efficiency profiles for the HRSE and HRSH respectively. For each water-foil, a solid angle cut has been used to select the flat region of the angular acceptance so that all the particles with the central momentum can reach the focal plane. For the central foil, the solid angle cut was $4.75 \mathrm{msr}$, whilr for the side foils, the cut changes with the spectrometer angle. As the

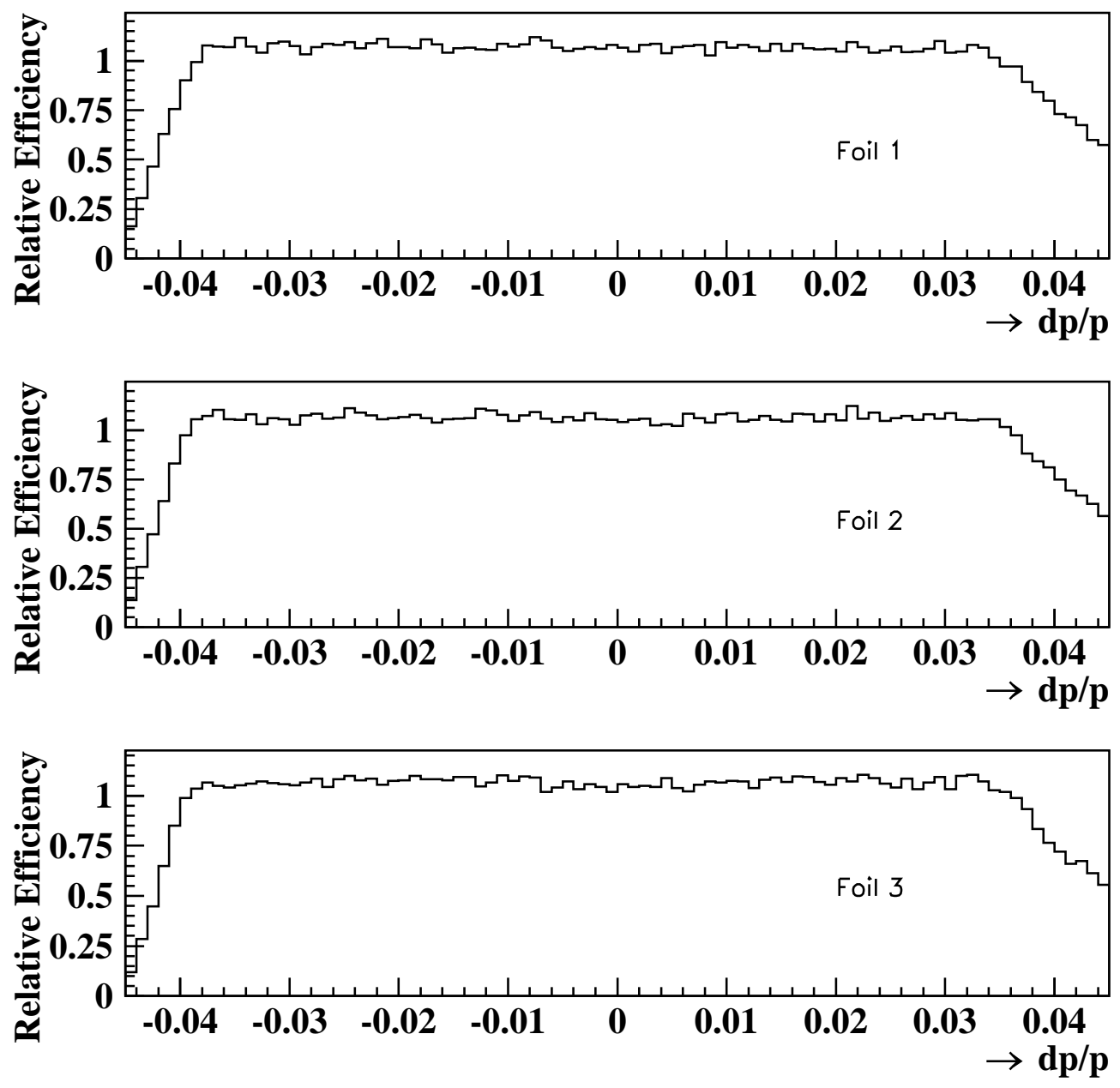

Figure 3-19: The relative efficiency profile of the HRSE focal plane for the three water-foils.

figures indicate, the relative efficiency profiles are flat over a $7 \%$ relative momentum range under the above solid angle cuts. For the analysis of the present experiment, only the data within the flat regions of acceptance was used. Table 3.3 presents the 

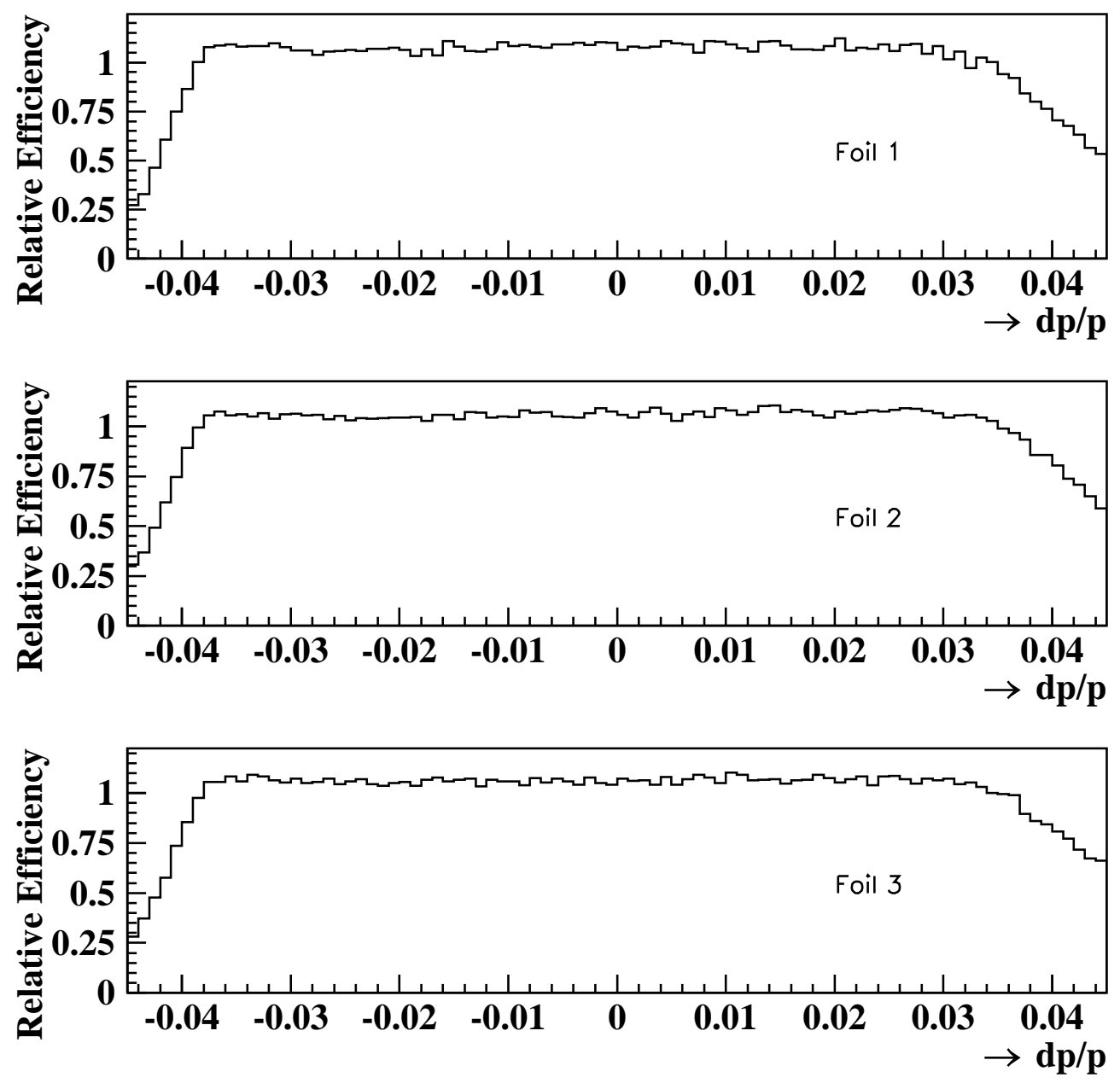

Figure 3-20: The relative efficiency profile of the HRSH focal plane for the three water-foils.

solid angle and relative momentum cuts used for all the data from this experiment.

\begin{tabular}{|c|c|c|}
\hline Variable & HRSE & HRSH \\
\hline Out of plane angle & $-50.0 \mathrm{mr}<\theta_{\mathrm{tg}}<45.0 \mathrm{mr}$ & $-50.0 \mathrm{mr}<\theta_{\mathrm{tg}}<50.0 \mathrm{mr}$ \\
\hline In plane angle & $-26.0 \mathrm{mr}<\phi_{\mathrm{tg}}<24.0 \mathrm{mr}$ & $-22.0 \mathrm{mr}<\phi_{\mathrm{tg}}<22.0 \mathrm{mr}$ \\
\hline Relative momentum & $-3.7 \%<\frac{d P}{P}<3.3 \%$ & $-3.7 \%<\frac{d P}{P}<3.3 \%$ \\
\hline
\end{tabular}

Table 3.3: Cuts used for the central water-foil to select the flat region of acceptance

The flat relative efficiency profiles combined with the absolute efficiency calculated at the center of the focal plane yields a $100.0 \% \pm 3.0 \%$ absolute efficiency for both spectrometers over the entire phase space defined by the above cuts for a thin target placed at the hall center. 


\section{Chapter 4}

\section{Cross section and beyond}

\subsection{Cross section calculation}

In $\left(e, e^{\prime} p\right)$ coincidence experiments, the cross section is measured over finite intervals of horizontal and vertical angular acceptance as well as energy for both the electron and the proton. As a result, the measured cross section is six-fold differential. For the present experiment, the data was binned in the four dimensional $\omega, Q^{2}, E_{m i s s}$, and $P_{\text {miss }}$ space. The six-fold differential cross section for a bin $B\left(\omega, Q^{2}, E_{\text {miss }}, P_{m i s s}\right)$ can be expressed by

$$
\frac{d^{6} \sigma}{d \omega d \Omega_{e} d E_{p} d \Omega_{p}}=\frac{N_{B}}{L \varepsilon_{e} \varepsilon_{p} \varepsilon_{\text {coin }} V_{B}} D R,
$$

where

$N_{B}$ is the number of particles scattered into the bin $B\left(\omega, Q^{2}, E_{\text {miss }}, P_{\text {miss }}\right)$,

$L$ is the luminosity, defined as the number of interactions per unit area of the target, $\varepsilon_{e}$ is the electron spectrometer efficiency,

the product $L \varepsilon_{e}$ is determined from the $\mathrm{H}\left(\mathrm{e}, \mathrm{e}^{\prime}\right)$ normalization as described in the next section.

$V_{B}$ is the phase space volume of the bin, $\varepsilon_{p}$ is the hadron spectrometer efficiency and $\varepsilon_{c o i n}$ is the coincidence trigger efficiency.

The determination of $\varepsilon_{p} \varepsilon_{c o i n}$ was described in section 3.6.4 $D$ is the dead-time correction, and 
$R$ is the radiative correction.

The number of scattered events $N_{B}$ in a bin was obtained by subtracting the accidental coincidences from the total number of events in the bin. This was performed using the corrected coincidence time-of-flight spectrum. Fig. 4-1 shows the coincidence time-of-flight spectrum divided into three separate regions. $W_{r}$ is the width of the region that includes the true coincidence peak, and $N_{r}$ is the number of events in this region. $W_{a 1}$ and $W_{a 2}$ are the widths of the two regions defined for the accidental coincidence events, and $N_{a}$ is the total number of events in these two regions. The number of true coincidence events $N_{t}$ is calculated as

$$
N_{t}=N_{r}-N_{a} \frac{W_{r}}{W_{a 1}+W_{a 2}} .
$$

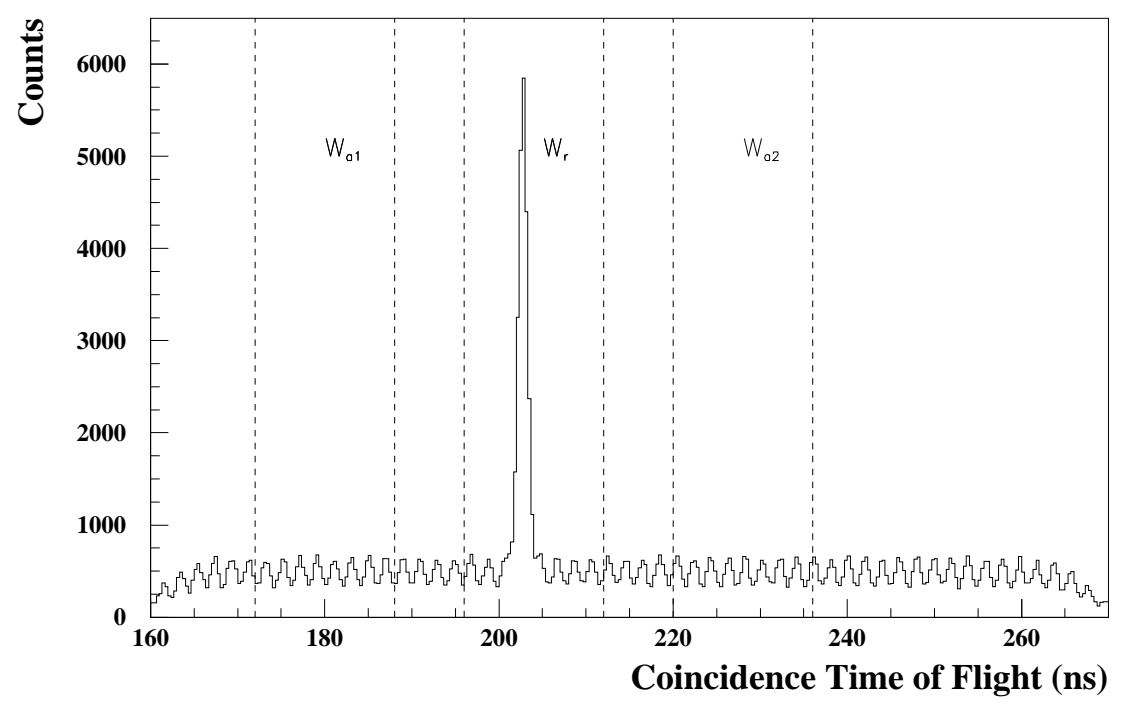

Figure 4-1: Corrected coincidence time-of-flight spectrum with the real and accidental regions defined for accidental subtraction.

\subsubsection{Luminosity calculation}

The luminosity for oxygen in the target can be expressed by

$$
L_{O}=\left(\frac{C}{e}\right)\left(\frac{N_{A}(\rho t)_{O}}{A}\right) \text {, }
$$


where

$C$ is the accumulated charge,

$e$ is the electron charge,

$N_{A}$ is Avagadro's number,

$A$ is the atomic weight, and

$(\rho t)_{O}$ is the effective thickness of oxygen in the target.

The luminosity is conveniently calculated for each run of this experiment through the $\mathrm{H}\left(\mathrm{e}, \mathrm{e}^{\prime}\right)$ normalization as described in section 3-17. For the $\mathrm{H}\left(\mathrm{e}, \mathrm{e}^{\prime}\right)$ reaction, the total cross section can be written as

$$
\frac{d \sigma_{H\left(e, e^{\prime}\right)}}{d \Omega_{e}}=\frac{e A}{N_{A}(\rho t)_{H} C \varepsilon_{e}} \frac{N R D}{\delta \Omega_{e}}
$$

where

$(\rho t)_{H}$ is the effective thickness of hydrogen in the target,

$\delta \Omega_{e}$ is the electron spectrometer solid angle,

$\varepsilon_{e}$ is the electron spectrometer efficiency,

$N$ is the number of events in the $\mathrm{H}\left(\mathrm{e}, \mathrm{e}^{\prime}\right)$ elastic peak integrated over an energy interval

$\Delta E$, and

$R$ is the radiative correction for the events radiated out of the interval $\Delta E$.

With the "world" cross section for the $\mathrm{H}\left(\mathrm{e}, \mathrm{e}^{\prime}\right)$ reaction calculated using the method outlined in section 3-17, the luminosity for $\mathrm{H}$ can be expressed by

$$
L_{H} \varepsilon_{e}=\left(\frac{C}{e}\right)\left(\frac{N_{A}(\rho t)_{H} \varepsilon_{e}}{A}\right)=\frac{N R D}{\sigma_{H\left(e, e^{\prime}\right)}^{\text {world }} \delta \Omega_{e}} .
$$

Since there are two hydrogen atoms for each oxygen atom in the target, the luminosity for oxygen is given by

$$
L_{O} \varepsilon_{e}=\frac{L_{H} \varepsilon_{e}}{2}=\frac{N R D}{2 \sigma_{H\left(e, e^{\prime}\right)}^{w o r l d} \delta \Omega_{e}} .
$$




\subsubsection{Phase-space volume calculation}

A Monte-Carlo simulation was performed to calculate the phase space volume for each bin. A total of $N_{0}^{s i m}$ events were generated, randomly distributed over the variables $E_{f}^{e}, \theta_{t g}^{e}, \phi_{t g}^{e}, E_{f}^{p}, \theta_{t g}^{p}$ and $\phi_{t g}^{p}$, within the flat region of the acceptance. For each event generated, the kinematic variables $\omega, Q^{2}, E_{m i s s}$ and $P_{\text {miss }}$ were calculated. Then the simulated events were binned in the $\omega, Q^{2}, E_{\text {miss }}, P_{\text {miss }}$ space. If the number of events in the bin $B\left(\omega, Q^{2}, E_{m i s s}, P_{m i s s}\right)$ is $N_{B}^{s i m}$, the phase-space volume for that bin is given by

$$
V_{B}=\frac{N_{B}^{s i m}}{N_{0}} \Delta E_{f}^{e} \Delta \Omega_{e} \Delta E_{f}^{p} \Delta \Omega_{p}
$$

Where $\Delta E_{f}^{e}, \Delta \Omega_{e}, \Delta E_{f}^{p}, \Delta \Omega_{p}$ are the ranges of variables $E_{f}^{e}, \Omega_{e}, E_{f}^{p}, \Omega_{p}$ considered for the simulation.

\subsubsection{Dead time correction}

As described in section 2.2.8, only a part of the event triggers are accepted and written to the data stream due to the dead time of the data acquisition system. The number of raw event triggers of each trigger type is counted by scaler modules. The number of triggers of each type written to the data stream is counted by ESPACE during data analysis. For the coincidence events, the dead time correction factor $D$ is calculated as

$$
D=\frac{S_{5}}{T_{5}}
$$

where $S_{5}$ is the total number of event triggers generated, and $T_{5}$ is the number of events written to the data stream. 


\subsection{Radiative Corrections}

Unfortunately, the (e,e'p) reaction never proceeds through the simple, one photon exchange diagram shown in Fig. 4-2. In reality, the incoming and the outgoing charged particles radiate real and virtual photons. This changes not only the cross section for the reaction, but also the apparent energy and the momentum transfer. Further, while these radiative processes are an integral part of the (e,e'p) reaction, theoretical work simply does not include these effects. Therefore, it is the job of the experimentalist to correct the measured cross sections for radiative losses in order to perform a meaningful comparison between the experiment and theory. In the correction process the measured cross section is adjusted to the values which would have been measured in the absence of radiation.

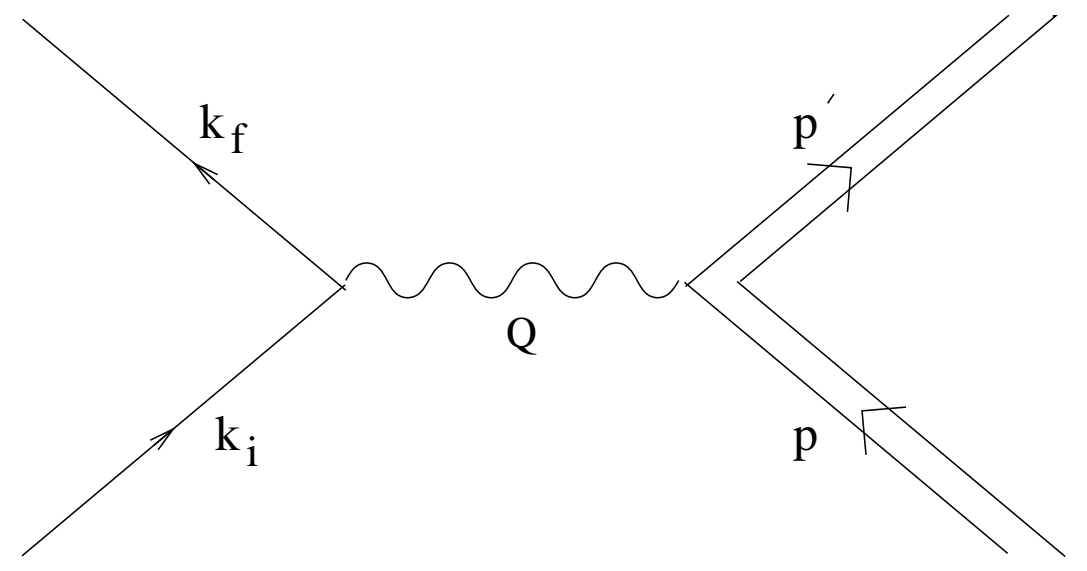

Figure 4-2: The (e,e'p) reaction in the first-order Born approximation.

The radiative corrections for the present experiment were performed with the RADCOR program, originally written at NIKHEF by E. Quint [66] and later modified at Mainz and at MIT. The program was modified to meet the requirements of the present experiment. The exact modifications are presented in the following sections.

Radiative processes may be grouped into two categories:

- Internal radiation: The electron radiates real and virtual photons in the presence of the Coloumb field of the target nucleus involved in the $\left(\mathrm{e}, \mathrm{e}^{\prime} \mathrm{p}\right)$ reaction.

- External radiation: The electron radiates real and virtual photons in the presence of the Coloumb fields of nuclei other than the target nucleus. 


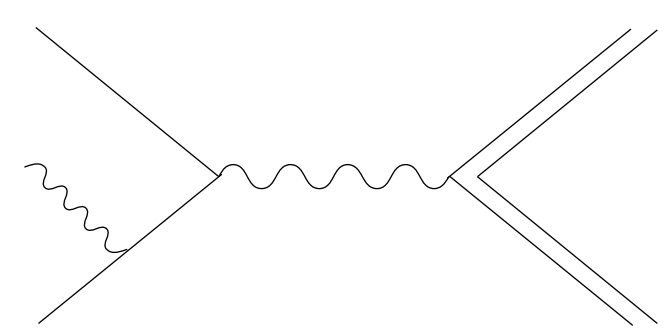

a

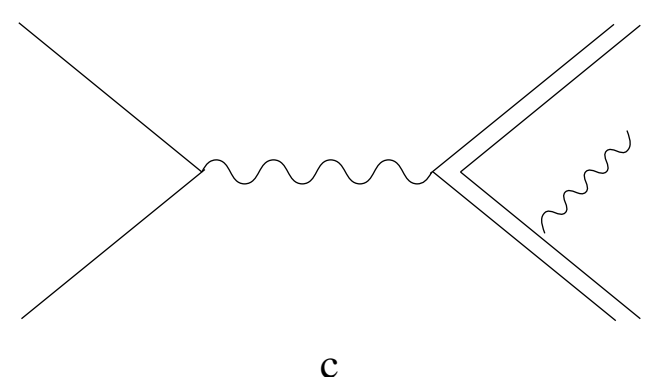

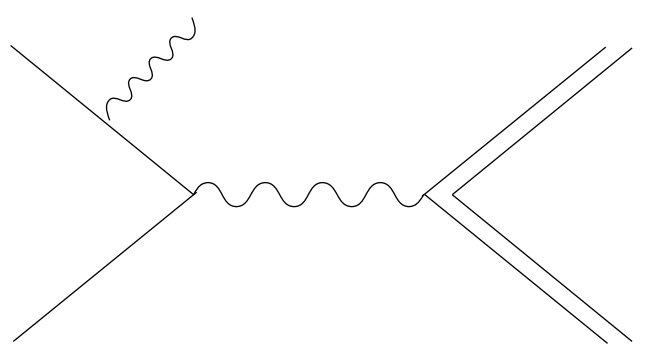

b

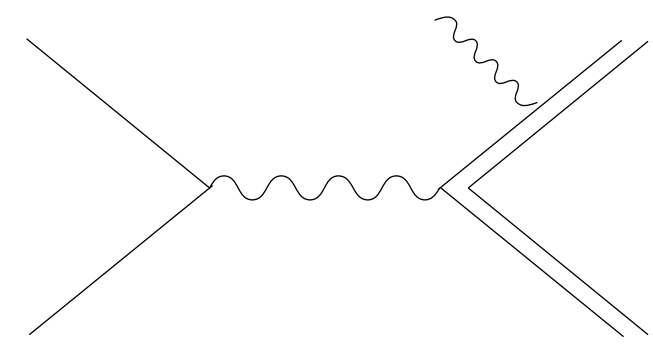

d

Figure 4-3: Feynman diagrams for the radiation of a single real photon.

The magnitude of the external radiative correction is proportional to the amount of material the electron has to traverse. Since a thin waterfall target was used for this experiment, the external corrections were smaller than the internal corrections. For different kinematic settings of the experiment, for radiating out of a $5 \mathrm{MeV}$ bin sampled across the ground state of ${ }^{16} \mathrm{O}$, the external corrections ranged from $5 \%$ to $10 \%$, while the internal corrections ranged from $35 \%$ to $55 \%$. In the following sections, the two radiative processes are described in detail.

\subsubsection{Internal radiation}

The four Feynman diagrams in Fig. 4-3 represent the processes that involve the radiation of a single bremsstrahlung photon. The emission of a real photon changes the four momenta of the incoming (or outgoing) charged particles. The emission of a virtual photon can only change the magnitude of the measured cross section, without altering the momenta of the particles. Fig. 4-4 presents a sample of virtual photon correction terms.

Schwinger [67] first formulated the radiative corrections for an electron scattering from a potential. In his formulation, the experimentally measured cross section is 
related to the one photon exchange (lowest order Born) cross section by

$$
\left(\frac{d \sigma}{d \omega}\right)_{e x p}=(1+\delta)\left(\frac{d \sigma}{d \omega}\right)_{B o r n},
$$

where

$$
\delta \cong-\frac{2 \alpha}{\pi}\left[\left(\ln \frac{E_{0}}{\Delta E}-\frac{12}{13}\right)\left(\ln \frac{-q^{2}}{m^{2}}-1\right)+\frac{17}{36}\right]
$$

In the same paper, Schwinger noted the presence of the so called infra-red $d i$ vergence in this formulation, that is, as $\Delta E \rightarrow 0, \delta \rightarrow \infty$. This divergence arose from the fact that Schwinger did not include multiple photon emission in his calculations. He suggested that the $(1+\delta)$ term in Eqn. 4.9 should be replaced by $e^{\delta}$ if multiple photon emissions were to be included. Later Yennie and Suura [68] proved that in fact the infra-red divergence goes away when the divergent part of $\delta:(-2 \alpha / \pi)\left[\left(-Q^{2} / m^{2}\right)-1\right] \ln (E / \Delta E)$, is exponentiated.

In the energy regime of $M_{p}^{2} \gg-Q^{2}$ where $M_{p}$ is the rest mass of the proton, Eqn 4.9 is a very good approximation. However, the advent of higher energy accelerators (which have enabled experiments with $M_{p}^{2}<-Q^{2}$ ) has demanded the consideration of factors like the target recoil and the radiation from the proton. Tsai [69] was the first to modify $\delta$ to include these two effects. Eqn. (II.6) of ref. [70] gives the modified equation for $\delta$. He included all four diagrams shown in Fig. 4-3, as well as the virtual photon contributions from the diagrams in Fig. 4-4.

For the kinematics of the present experiment, the contribution from the proton radiation is relatively small. The formulae ${ }^{1}$ derived in reference [71] for ultra-relativistic particles have been used to estimate the relative strength of the proton radiation. In the worst case, the contribution to the corrected cross section due to radiation from the proton is about $1 \%$. Since this is well within the systematic and statistical uncertainties of this experiment, this radiation has been neglected in this analysis. However, it should be noted that as one goes to higher values of $Q^{2}$, the proton contributions increase. Therefore, the relative strength of these contributions must always be considered.

\footnotetext{
${ }^{1}$ Eqns. 4.42 of reference [71]
} 


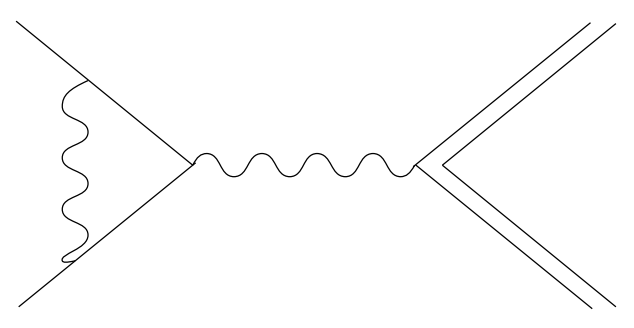

a

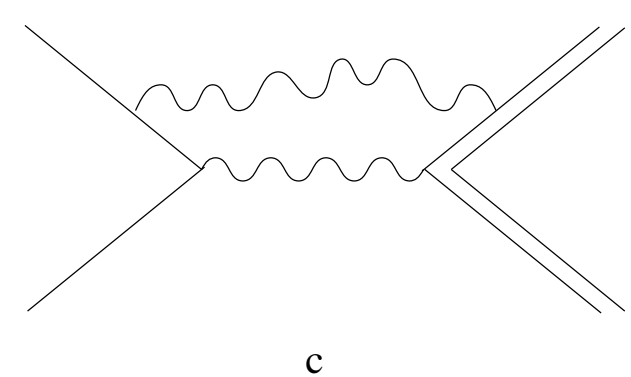

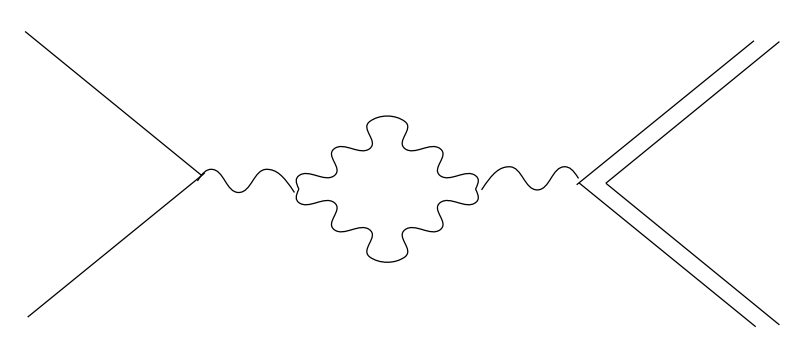

b

Figure 4-4: A few of the Feynman diagrams for the virtual photon corrections to the one photon exchange cross section.

Many approximate formulae based on Tsai's prescription are available in the literature. In RADCOR, the formulation by Penner [72] is employed. In Penner's formulation, $\delta$ is given by (neglecting radiation from the proton),

$$
\begin{gathered}
\delta=\delta_{\text {real }}+\delta_{\text {virtual }} \\
\delta_{\text {real }}=\frac{\alpha}{\pi}\left[\ln \left(\frac{E_{0} E^{\prime} b}{\eta^{2} \Delta \mathrm{E}^{2}}\right)\right]\left[\ln \left(\frac{-q^{2}}{\mathrm{~m}^{2}}\right)-1\right], \text { and } \\
\delta_{\text {virtual }}=\frac{\alpha}{\pi}\left\{\frac{13}{6}\left[\ln \left(\frac{-q^{2}}{\mathrm{~m}^{2}}\right)-1\right]-\frac{17}{18}-\frac{\pi^{2}}{6}-\frac{1}{2} \ln ^{2}\left(\frac{E_{0}}{\mathrm{E}^{\prime}}\right)+\mathrm{L}_{2}\left(\cos ^{2}\left(\frac{\theta}{2}\right)\right)\right\} .
\end{gathered}
$$


In the above equations

$$
\begin{aligned}
& b=1+\frac{2 \omega}{M} \sin ^{2}\left(\frac{\theta}{2}\right), \\
& \eta=1+\frac{2 E^{\prime}}{M} \sin ^{2}\left(\frac{\theta}{2}\right),
\end{aligned}
$$

and $L_{2}$ is the Spence Function

$$
L_{2}(x)=\int_{0}^{x} \frac{\ln (1-y)}{y} d y \text {. }
$$

$\delta_{\text {real }}$ is due to the emission of real photons by the incoming electron (diagrams (a) and (b) in Fig. 4-3), while $\delta_{\text {virtual }}$ represents virtual photon corrections. $\delta_{\text {real }}$ is divergent due to the presence of $\Delta E$ in the denominator, while $\delta_{\text {virtual }}$ is non-divergent. Thus, the lowest order Born cross section can be related to the measured cross section by

$$
\left(\frac{d \sigma}{d \omega}\right)_{b o r n}=\left(\frac{d \sigma}{d \omega}\right)_{\text {exp }}\left(\frac{e^{\delta_{\text {real }}}}{1-\delta_{\text {virtual }}}\right)
$$

In RADCOR, $\delta_{\text {real }}$ and $\delta_{\text {virtual }}$ are combined as the total Schwinger correction term

$$
C_{S c h w}=\frac{e^{\delta_{\text {real }}}}{1-\delta_{\text {virtual }}}
$$

\subsubsection{External radiation}

RADCOR uses the formalism of Friedrich [73] for the external radiative correction.

When an electron travels a distance $t$ through a material with radiation length $X_{0}$, the probability of radiating one real photon can be written as

$$
f^{r a d}=\frac{t}{X_{0}} \frac{1}{\Delta E}\left[1-\eta \frac{E_{f}}{E_{i}}+\left(\frac{E_{f}}{E_{i}}\right)^{2}\right],
$$

where

$$
\eta=\frac{2}{3}-\frac{1}{9}\left(\ln 183 \mathrm{Z}^{-\frac{1}{3}}\right)^{-1}
$$

This probability density may be used to compute an approximate radiative cor- 
rection $K_{\text {ext }}^{a p p r o x}$ for the energy interval $\Delta E$ according to,

$$
\sigma_{\text {exp }}(\Delta E)=K_{\text {ext }}^{a p p r o x}(\Delta E) \cdot \sigma_{\text {Born }}(\Delta E)
$$

where

$$
\begin{aligned}
K_{\text {ext }}^{\text {approx }}(\Delta E) & \approx 1-\int_{0}^{E_{i}-\Delta E} \frac{t}{X_{0}\left(E_{i}-E_{f}\right)}\left[1-\eta \frac{E_{f}}{E_{i}}+\frac{E_{f}^{2}}{E_{i}^{2}}\right] d E_{f} \\
& =1-\delta^{e x t}
\end{aligned}
$$

After integration, $\delta^{e x t}$ is given by

$$
\delta^{e x t}=\frac{t}{X_{0}}\left[-\left(\frac{1}{2}-\eta\right)+(2-\eta) \ln \left(\frac{E_{i}}{\Delta \mathrm{E}}\right)+(2-\eta)\left(\frac{\Delta \mathrm{E}}{\mathrm{E}_{\mathrm{i}}}\right)-\frac{\Delta \mathrm{E}^{2}}{2 \mathrm{E}_{2}^{2}}\right]
$$

$K_{\text {ext }}^{\text {approx }}$ yields unphysical results as $\Delta E \rightarrow 0$. To correct for this, the same argument Schwinger used to avoid the infrared divergence in the internal radiation case is used

$$
K_{\text {ext }}^{\text {approx }}=e^{-\delta}
$$

where, in the notation of RADCOR,

$$
C_{\text {ext }}=\frac{1}{K_{\text {ext }}^{\text {approx }}}=e^{\delta}
$$

In addition to internal and external bremsstrahlung corrections, Landau straggling (the energy loss due to inelastic collisions with atomic electrons of the target) is also taken into account using the formalism given by Findlay and Dusautoy [74]. For the present experiment, the magnitude of this effect is negligible for the incoming and scattered electrons, and amounts to less than $1 \%$ for the scattered protons.

The correction factors previously discussed may be combined into a single expression representing the correction for radiating a photon out of an energy interval $\Delta E$ as

$$
C(\Delta E)=C_{S c h w}(\Delta E) \cdot C_{\text {ext }}(\Delta E) \cdot C_{\text {Land }}(\Delta E)
$$


with

$$
\sigma_{\text {Born }}(\Delta E)=C(\Delta E) \cdot \sigma_{\text {exp }}(\Delta E)
$$

\subsubsection{Radiative unfolding procedure for $\left(e, e^{\prime} p\right)$ experiments}

Analysis of an (e,e'p) experiment involves sampling data in a multi-dimensional hyperspace. In the case of the present experiment, the data was sampled in a 4-D $\left(E_{m i s s}, P_{m i s s}, \omega, Q^{2}\right)$ space. The radiative correction procedure should take into account the propagation of radiative tails in this hyperspace. Things are further complicated by having to consider radiation from all three particles involved in the reaction, $\mathrm{e}, \mathrm{e}^{\prime}$ and $\mathrm{p}^{2}$. The radiation from these particles not only changes the variables $\omega$ and $E_{m i s s}$ calculated for the reaction, but it also changes the $\vec{P}_{m i s s}$ and $\vec{q}$ vectors.

In general, the radiated photon can be emitted in any direction, making it difficult to evaluate the effect of this radiation on the direction of the $\vec{P}_{m i s s}$ and $\vec{q}$ vectors. Fortunately, at the ultra-relativistic energy regime of the present experiment, the peaking approximation is very accurate. According to the peaking approximation, the photon is emitted in the same direction the particle which radiated it was traveling. This approximation has been used to derive the expressions for the change in $P_{m i s s}$ in Eqns. 4.30 through 4.32.

For the radiation of a photon of energy $E_{\gamma}$ by e, $\mathrm{e}^{\prime}$ or $\mathrm{p}$, the missing energy and missing momentum are modified in the following manner:

$$
\begin{aligned}
E_{m 1,2,3} & =E_{m}+T_{r e c}-T_{r e c}^{\text {meas }}+E_{\gamma} \\
& \cong E_{m}+E_{\gamma} \\
\vec{p}_{m 1} & =\vec{p}_{m 0}+E_{\gamma} \hat{\mathbf{k}} \\
\vec{p}_{m 2} & =\vec{p}_{m 0}+E_{\gamma} \hat{\mathbf{k}^{\prime}} \\
\vec{p}_{m 3} & =\vec{p}_{m 0}+E_{\gamma} \frac{E_{p}}{p} \hat{\mathbf{p}}
\end{aligned}
$$

\footnotetext{
${ }^{2}$ As mentioned before, the internal radiation from the initial and the scattered protons is negligible for the kinematics of the present experiment. However, the Landau straggling losses from the scattered proton are considered.
} 
where the indices 1, 2 and 3 indicate radiation from particles e, $\mathrm{e}^{\prime}$ and p respectively. In the above equations, $\hat{\mathbf{k}}, \hat{\mathbf{k}}^{\prime}$ and $\hat{\mathbf{p}}$ are unit vectors along the directions of $\mathrm{e}, \mathrm{e}^{\prime}$ and $\mathrm{p}, E_{p}$ is the energy of the ejected proton, $T_{r e c}$ is the kinetic energy of the recoiling nucleus, and $T_{\text {rec }}^{\text {meas }}$ is the measured kinetic energy of the recoiling nucleus. The difference between $T_{r e c}$ and $T_{\text {rec }}^{\text {meas }}$ results from the change in the missing momentum due to radiative effects. This difference is negligible for ${ }^{16} \mathrm{O}$.

Cross section data in a convenient set of basis variables is supplied as input for RADCOR. This can be the 4-D $\left(E_{m i s s}, \vec{P}_{m i s s}\right)$ space, 2-D $\left(E_{m i s s},\left|\vec{P}_{m i s s}\right|\right)$ space, or as in the case of the present experiment, the 4-D $\left(E_{m i s s},\left|\vec{P}_{m i s s}\right|, \omega, Q^{2}\right)$ space. Since the magnitude and direction of the radiative tail depend on $\overrightarrow{k^{\prime}}$ and $\vec{p}$, it is necessary that these vectors may be reconstructed from the input variables. Combined with azimuthal symmetry about the beam axis, five variables suffice to uniquely determine $\overrightarrow{k^{\prime}}$ and $\vec{p}$. However, only four variables are available.

Several approaches may be used to circumvent this problem:

- The traditional approach of RADCOR was to use a five-dimensional input with either $|\vec{q}|$ or $\omega$ being the fifth variable. This method requires statistics adequate to bin data in five dimensions.

- In case of the present experiment, the added restriction that the scattering is in-plane $(\phi=0)$ is used. With the relatively small out-of-plane acceptance of the HRS pair $( \pm 50.0 \mathrm{mr})$, the uncertainty associated with this restriction is negligible.

For clarity and convenience of presentation, the correction procedure is presented in the 2-D $E_{\text {miss }},\left|\vec{P}_{\text {miss }}\right|$ space. Fig. 4-5 shows the propagation of the radiative tail due to e, $\mathrm{e}^{\prime}$ and $\mathrm{p}$ for two kinematic settings on either side of $\vec{q}$. In order to correct for these tails, a method based on the radiative unfolding suggested by Crannell [75] is used. The correction procedure is initiated at the $\left|\vec{P}_{m i s s}\right|$ bins with the lowest $E_{m i s s}$, (for the present case $E_{\text {miss }}=10.0 \mathrm{MeV}$ ). Since there is nothing radiating into these bins, a correction is needed only for the strength that radiated out.

The following procedure is used to correct all the bins in the grid starting with the lowest $E_{m i s s}$ bins. Note that $i$ refers to the $E_{\text {miss }}$ bin radiating out (losing events) 

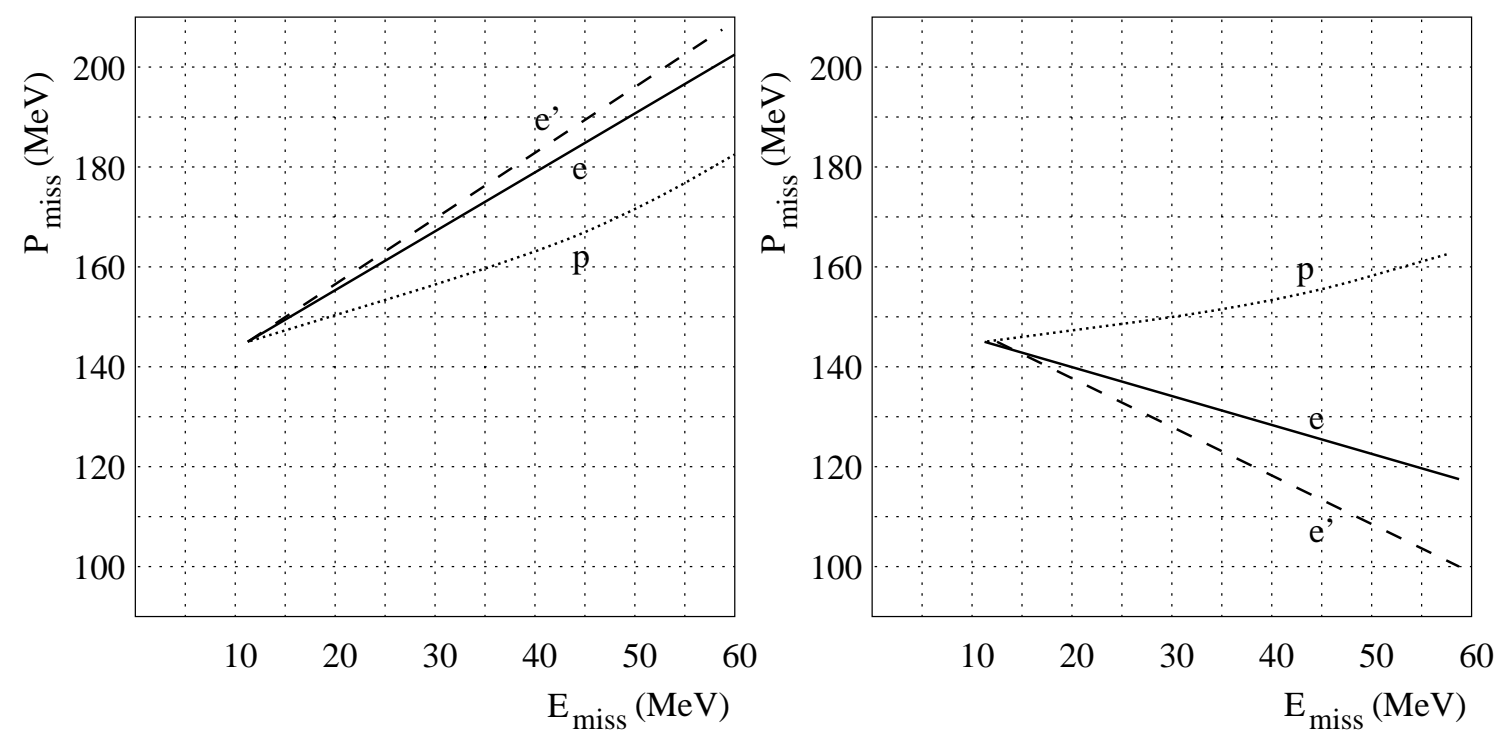

Figure 4-5: Radiative tails due to e, $\mathrm{e}^{\prime}$ and $\mathrm{p}$. The plots are from $E_{0}=2442.3 \mathrm{MeV}$ settings. The left plot corresponds to $\theta_{p q}=+8.0^{\circ}$ and the right plot corresponds to $\theta_{p q}=-8.0^{\circ}$.

and $j$ refers to the $E_{m i s s}$ bin radiating in (receiving events from bin $i$ )

1. Correct the measured strength at the lowest $\left|\vec{P}_{\text {miss }}\right|$ cell of the current $E_{\text {miss }}$ channel for the radiative losses according to:

$$
\sigma_{\text {Born }}\left(i,\left|\vec{P}_{\text {miss }}\right|\right)=\sigma_{\text {exp }}\left(i,\left|\vec{P}_{\text {miss }}\right|\right) \cdot C_{\text {Schw }} \cdot C_{\text {ext }} \cdot C_{\text {Land }}
$$

2. Calculate the radiative tail from the current cell to all the higher $E_{m i s s}$ channels. The tail contribution to the $j^{\text {th }}$ channel may be written as

$$
\Delta \sigma_{1,2,3}\left(j, p_{m_{a}}\right)=\bar{\sigma}_{1,2,3}\left(i, p_{m_{b}}\right)\left[\frac{1}{C_{(\Delta E(j-i))}^{(1,2,3)}}-\frac{1}{C_{(\Delta E(j-i-1))}^{(1,2,3)}}\right],
$$

where the indices 1, 2 and 3 stand for the radiation from $\mathrm{e}, \mathrm{e}^{\prime}$ and $\mathrm{p}$ respectively, and $\Delta E$ is the bin width in missing energy.

Events can radiate into all three tails at the same time. As a result, an average value $\bar{\sigma}_{1,2,3}\left(i, p_{m}\right)$ is used for the cross section in the cell, when the radiation took place in the corresponding tail. This average cross section is calculated by taking into account all $3 !=6$ tail propagation sequences. 


$$
\begin{aligned}
& \bar{\sigma}_{1}\left(i, p_{m}\right)=\frac{\sigma\left(i, p_{m}\right)}{6}\left[2+\frac{1}{C_{\Delta E}^{(2)}}+\frac{1}{C_{\Delta E}^{(3)}}+\frac{2}{C_{\Delta E}^{(2)} C_{\Delta E}^{(3)}}\right] \\
& \bar{\sigma}_{2}\left(i, p_{m}\right)=\frac{\sigma\left(i, p_{m}\right)}{6}\left[2+\frac{1}{C_{\Delta E}^{(1)}}+\frac{1}{C_{\Delta E}^{(3)}}+\frac{2}{C_{\Delta E}^{(1)} C_{\Delta E}^{(3)}}\right] \\
& \bar{\sigma}_{3}\left(i, p_{m}\right)=\frac{\sigma\left(i, p_{m}\right)}{6}\left[2+\frac{1}{C_{\Delta E}^{(2)}}+\frac{1}{C_{\Delta E}^{(1)}}+\frac{2}{C_{\Delta E}^{(2)} C_{\Delta E}^{(1)}}\right]
\end{aligned}
$$

The direction of propagation of these tails in the $E_{m i s s},\left|\vec{P}_{m i s s}\right|$ plane may be calculated using eqns. 4.29 - 4.32. The directions of propagation of the three tails due to e, $\mathrm{e}^{\prime}$, and $\mathrm{p}$ allow for the calculation of $\left|\vec{P}_{m i s s}\right|$ bins at each higher $E_{m i s s}$ channel, into which the radiative tail from the current bin has gone. The tail contributions are then subtracted from the strengths of those bins.

3. Go to higher $\left|\vec{P}_{m i s s}\right|$ bins at the current $E_{m i s s}$ channel and repeat steps 1 and 2 . 4. Go to higher $E_{\text {miss }}$ channels and repeat steps 1,2 and 3.

One major problem faced by the radiative unfolding procedure is the tails coming into the measured region of the phase space from the regions outside. Without the knowledge of the cross section that contribute these tails we can not use the procedure outlined above. The traditional approach of RADCOR to solve this problem was to extrapolate the measured strength out of the measured region and then use this extrapolated strength to calculate the tails coming in from outside. 


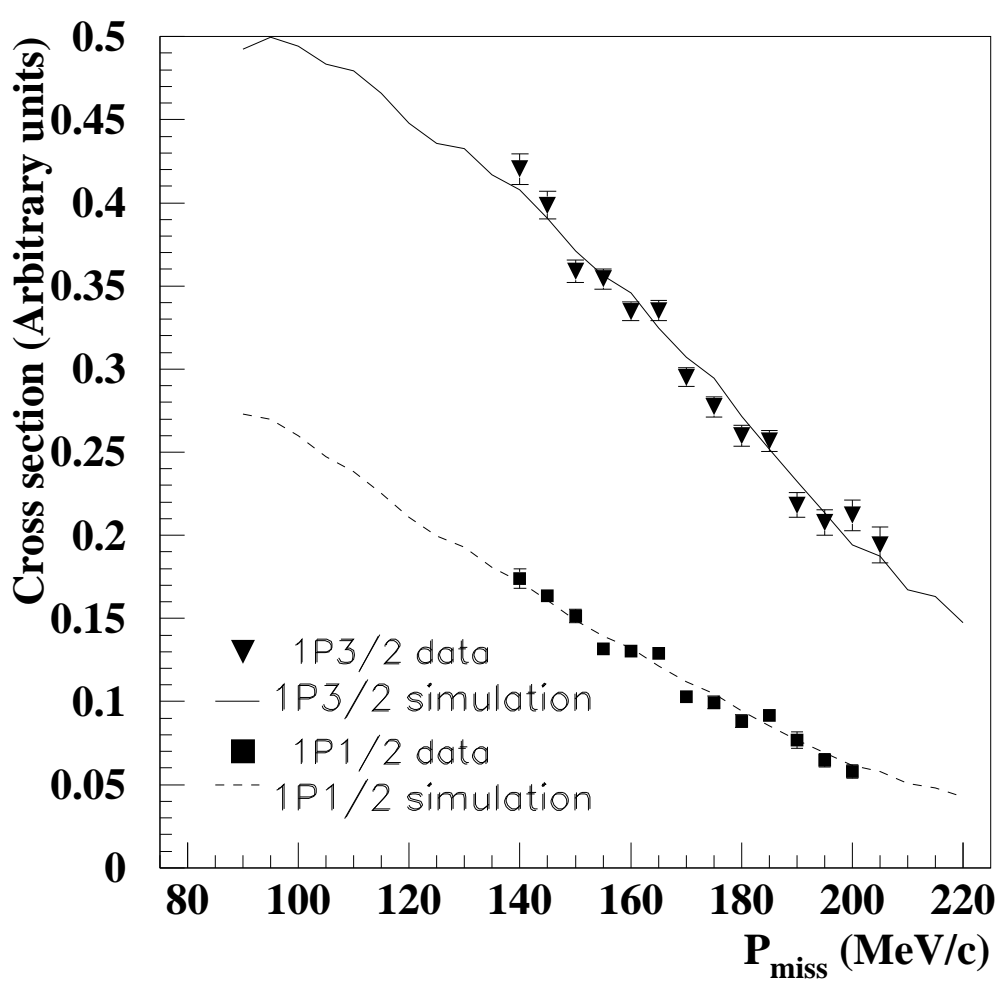

Figure 4-6: Momentum distribution simulations from MCEEP based on PWIA calculation by Van Orden, normalized to data. $E_{0}=2.445 \mathrm{GeV}, \theta_{p q}=8^{\circ}$ kinematic setting.

For the analysis of the present experiment, a different method was used to handle the unmeasured regions. A PWIA momentum distribution calculated by Van Orden was combined with the Monte-Carlo simulation package MCEEP, to generate the cross sections for $1 \mathrm{p}_{1 / 2}, 1 \mathrm{p}_{3 / 2}$, and $1 \mathrm{~s}_{1 / 2}$ states as a function of missing momentum for every kinematic setting of the experiment. The generated cross sections were normalized to the measured cross sections within the measured region. Then these normalized cross sections outside the measured region were used to calculate the tails radiating in. Fig. 4-6 shows the normalized momentum distributions for the $1 \mathrm{p}_{1 / 2}$ and $1 \mathrm{p}_{3 / 2}$ states for one of the kinematics settings of the experiment.

Fig. 4-7 shows the measured cross section before and after radiative corrections. The correction was performed in the $4 \mathrm{D}\left(E_{m i s s},\left|\vec{P}_{m i s s}\right|, \omega, Q^{2}\right)$ space. For the purpose of presentation, the cross section has been projected onto the $E_{m i s s}$ axis. 

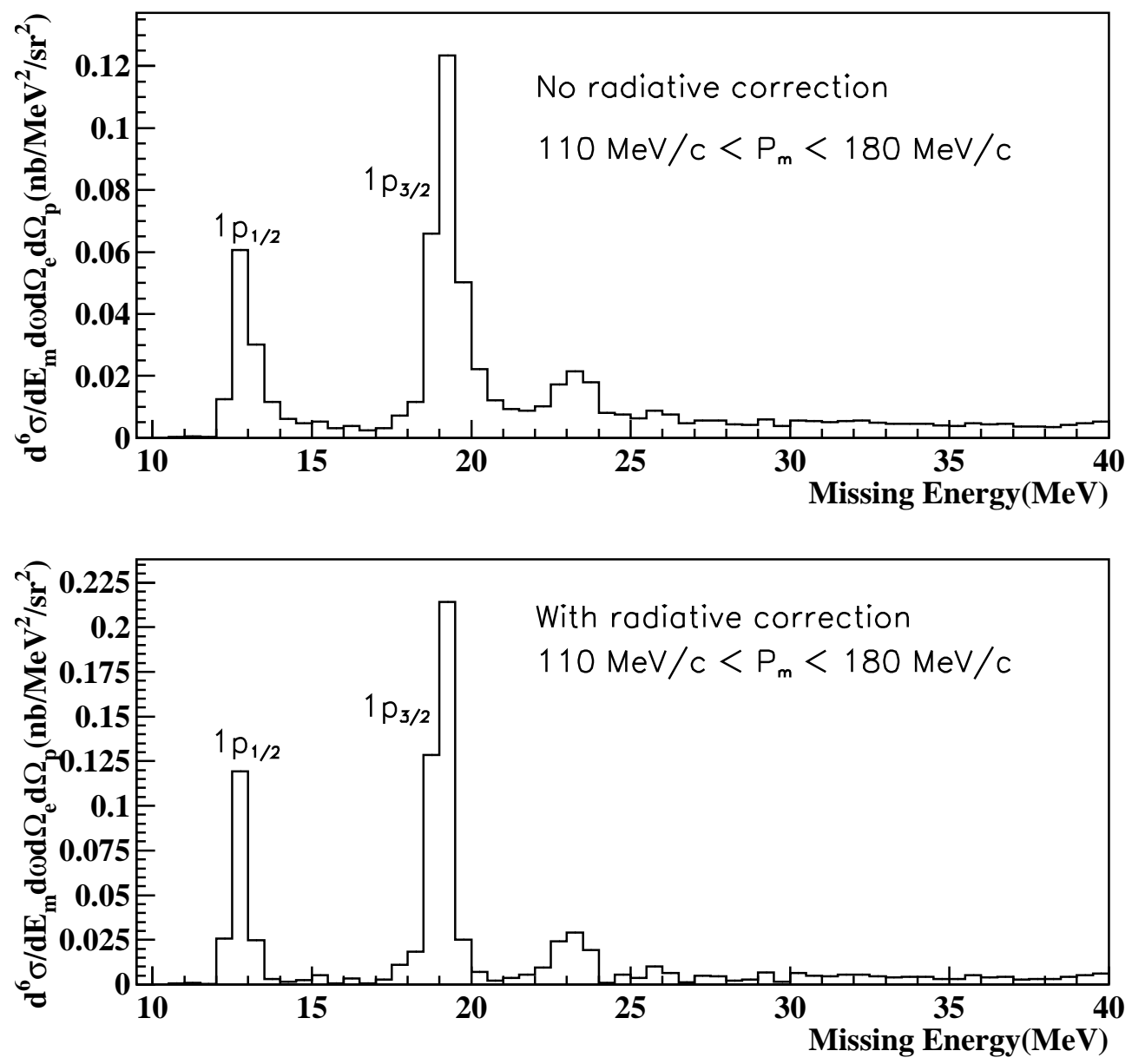

Figure 4-7: ${ }^{16} \mathrm{O}\left(\mathrm{e}, \mathrm{e}^{\prime} \mathrm{p}\right)$ cross section for $\theta_{p q}=+8^{\circ}$ at $\mathrm{E}_{\text {beam }}=0.8433 \mathrm{GeV}$. 


\subsection{Cross section calculation procedure}

The steps used in calculating the cross sections for this experiment are as follows:

1. ESPACE was used to analyze the raw data and to calculate the following variables for each event:

- $\theta_{t g}, \phi_{t g}, y_{t g}, \frac{\Delta p}{p}$ and the absolute momentum for each spectrometer.

- $E_{\text {miss }},\left|\vec{P}_{\text {miss }}\right|, \omega$ and $Q^{2}$.

- Corrected coincidence time-of-flight.

These variables were written into an $h b o o k^{3}$ ntuple for further analysis by FORTRAN codes written for the cross section calculation and the response function separation.

2. Cuts were placed on the variables $\theta_{t g}, \phi_{t g}, y_{t g}$, and $\frac{\Delta p}{p}$ calculated for each spectrometer such that the accepted events were within the flat region of each of these eight variables. The cuts used for this analysis were presented earlier in table 3.3.

3. The data were binned in the $E_{m i s s},\left|\vec{P}_{m i s s}\right|, \omega$ and $Q^{2}$ space. For the analysis of the data presented in this thesis, the $E_{\text {miss }}$ and $\left|\vec{P}_{\text {miss }}\right|$ bin size was selected to be $5 \mathrm{MeV}$. The bin sizes for $\omega$ and $Q^{2}$ were determined based on the available phase space volumes.

4. From the total number of events in a given bin the accidental coincidences were subtracted as described earlier.

5. The phase-space Monte-Carlo was performed under the same acceptance cuts used for the data, and the simulated events were binned using the same binning used for the data.

6. The bin with the largest phase-space volume $V^{\max }$ was found. Only the bins with phase-space volumes larger than $50 \%$ of $V^{\max }$ were used for further analysis.

\footnotetext{
${ }^{3}$ hbook is the histograming package used by CERN program libraries [76].
} 
7. The cross section for each bin was calculated by using Eqn. 4.1. The statistical error for the cross section in each bin is given by

$$
\frac{\delta \sigma_{B}}{\sigma_{B}}=\frac{1}{\sqrt{N_{r}+\left(\frac{W_{r}}{W_{a}}\right)^{2} N_{a}}},
$$

where

$N_{r}$ is the number of events in the bin that fall within the real window of the TOF spectrum,

$N_{a}$ is the total number of events that fall within the accidental windows,

$W_{r}$ is the width of the real window, and

$W_{a}$ is the total width of the accidental windows.

8. The average cross section for a given kinematic setting was calculated as a function of missing energy. The cross sections calculated for all the selected bins with a given missing energy were averaged according to

$$
\sigma=\frac{\sum_{B} \sigma_{B}}{\sum_{B}},
$$

with an uncertainty given by

$$
\delta \sigma=\frac{\left.\sqrt{\sum_{B}\left(\delta \sigma_{B}\right.}\right)^{2}}{\sum_{B}} .
$$




\subsection{Response function calculation}

The response functions, $R_{L}, R_{T}, R_{T T}$ and $R_{L T}$ were defined in chapter 1 by the equation

$$
\frac{d^{6} \sigma}{d \Omega_{e} d \Omega_{p} d \omega d E_{p^{\prime}}}=K \sigma_{M}\left(V_{\mathrm{L}} R_{\mathrm{L}}+V_{\mathrm{T}} R_{\mathrm{T}}+V_{\mathrm{LT}} R_{\mathrm{LT}} \cos \phi+V_{\mathrm{TT}} R_{\mathrm{TT}} \cos 2 \phi\right)
$$

where

$$
\begin{aligned}
K & =\frac{p^{\prime} E_{p^{\prime}}}{(2 \pi)^{3}}, \\
\sigma_{M} & =\frac{\alpha^{2} \cos ^{2}\left(\theta_{e} / 2\right)}{4 E_{i}^{2} \sin ^{4}\left(\theta_{e} / 2\right)}, \\
V_{\mathrm{L}} & =\frac{Q^{4}}{\vec{q}^{4}}, \\
V_{\mathrm{T}} & =\frac{Q^{2}}{2 \vec{q}^{2}}+\tan ^{2}\left(\theta_{e} / 2\right), \\
V_{\mathrm{LT}} & =\frac{Q^{2}}{\vec{q}^{2}}\left[\frac{Q^{2}}{\vec{q}^{2}}+\tan ^{2}\left(\theta_{e} / 2\right)\right]^{\frac{1}{2}}, \\
V_{\mathrm{TT}} & =\frac{Q^{2}}{2 \vec{q}^{2}}
\end{aligned}
$$

\subsection{1 $R_{L T}$ calculation}

Data taken on either side of $\vec{q}$ for the same $\left|\theta_{p q}\right|$ and the same beam energy were used for the $R_{L T}$ calculation. This calculation was performed bin-by-bin in the 4dimensional $\left(E_{m i s s},\left|\vec{P}_{m i s s}\right|, \omega, Q^{2}\right)$ space. Each bin on one side of $\vec{q}$ was matched to the bin with the same $\left(E_{m i s s},\left|\vec{P}_{m i s s}\right|, \omega, Q^{2}\right)$ on the other side of $\vec{q}$. The phase-space volumes of both bins of a pair were checked. The pair was selected for the $R_{L T}$ calculation only if both bins had phase-space volumes larger than $50 \%$ of $V^{\max }$.

$R_{L T}$ is calculated from a selected pair using eqn $4.41 \mathrm{as}^{4}$,

$$
R_{L T}^{B}=\frac{\sigma^{B}\left(0^{\circ}\right)-\sigma^{B}\left(180^{\circ}\right)}{2 K^{B} V_{L T}^{B}},
$$

\footnotetext{
${ }^{4}$ For the convenience of the presentation the six-fold differential cross section $\frac{d^{6} \sigma}{d \Omega_{e} d \Omega_{p} d \omega d E_{p^{\prime}}}$ is denoted by $\sigma$ in the following equations.
} 
with

$$
K^{B}=\frac{E_{p} p_{p}}{(2 \pi)^{3}} \sigma_{M}
$$

where

$\sigma^{B}\left(0^{\circ}\right)$ is the cross section measured for the bin $B\left(\omega, Q^{2}, E_{m i s s},\left|\vec{P}_{m i s s}\right|\right)$ for $\phi=0^{\circ}$, $\sigma^{B}\left(180^{\circ}\right)$ is the cross section measured for the same bin for $\phi=180^{\circ}$, $E_{p}$ and $p_{p}$ are the energy and the momentum of the knocked-out proton respectively, $\sigma_{M}$ is the Mott cross section, and $V_{L T}^{B}$ is the kinematic quantity defined in the previous section calculated for the kinematics of the bin $B$.

The uncertainty in $R_{L T}$ is given by

$$
\delta R_{L T}^{B}=\frac{\sqrt{\left[\delta \sigma^{B}\left(0^{\circ}\right)\right]^{2}+\left[\delta \sigma^{B}\left(180^{\circ}\right)\right]^{2}}}{2 K^{B} V_{L T}^{B}} .
$$

For each $\theta_{p q}, R_{L T}$ was calculated as a function of missing energy. A linear average was taken over all the selected bins with a given missing energy

$$
R_{L T}\left(\theta_{p q}, E_{m i s s}\right)=\frac{\sum_{B} R_{L T}^{B}\left(\theta_{p q}, E_{m i s s}\right)}{\sum_{B}}
$$

with an uncertainty

$$
\delta R_{L T}\left(\theta_{p q}, E_{m i s s}\right)=\frac{\sqrt{\sum_{B} \delta R_{L T}^{B}\left(\theta_{p q}, E_{m i s s}\right)^{2}}}{\sum_{B}} .
$$

\subsection{2 $R_{L}+\frac{V_{T T}}{V_{L}} R_{T T}, R_{T}$ separation}

For the $E_{0}=2442.3 \mathrm{MeV}$ and $E_{0}=1642.5 \mathrm{MeV}$ beam energy settings, the cross sections were measured on either side of $\vec{q}$ for a given $\theta_{p q}$. For these settings, the cross sections of a matched pair of bins on either side of $\vec{q}$ can be added to give

$$
\Sigma=\frac{\sigma\left(0^{\circ}\right)+\sigma\left(180^{\circ}\right)}{2 K}=V_{L}\left(R_{L}+\frac{V_{T T}}{V_{L}} R_{T T}\right)+V_{T} R_{T} .
$$

and 


$$
\delta \Sigma=\frac{\sqrt{\left[\delta \sigma^{B}\left(0^{\circ}\right)\right]^{2}+\left[\delta \sigma^{B}\left(180^{\circ}\right)\right]^{2}}}{2 K}
$$

For the $E_{0}=843.3 \mathrm{MeV}$ nominal beam energy setting, the cross sections were measured only on one side of $\vec{q}$. For this case, $V_{L}\left(R_{L}+\frac{V_{T T}}{V_{L}} R_{T T}\right)+V_{T} R_{T}$ can be calculated by using $R_{L T}$ calculated for a different beam energy, but at the same values of $\left(\omega, Q^{2}, E_{\text {miss }},\left|\vec{P}_{m i s s}\right|\right)$.

Starting from eqn. 4.41, one can write

$$
\begin{aligned}
V_{L}\left(R_{L}+\frac{V_{T T}}{V_{L}} R_{T T}\right)+V_{L T} R_{L T}+V_{T} R_{T} & =\frac{\sigma\left(0^{\circ}\right)}{K}, \text { or } \\
V_{L}\left(R_{L}+\frac{V_{T T}}{V_{L}} R_{T T}\right)+V_{T} R_{T} & =\frac{\sigma\left(0^{\circ}\right)}{K}-V_{L T} R_{L T} .
\end{aligned}
$$

For this case,

$$
\Sigma=\frac{\sigma\left(0^{\circ}\right)}{K}-V_{L T} R_{L T}
$$

with

$$
\delta \Sigma=\sqrt{\frac{\left[\delta \sigma\left(0^{\circ}\right)\right]^{2}}{K^{2}}+V_{L T}^{2}\left(\delta R_{L T}\right)^{2}} .
$$

A plot of $\Sigma / V_{L}$ as a function of $V_{T} / V_{L}$ for the three beam energies but at the same values of $\left(\omega, Q^{2}, E_{m i s s},\left|\vec{P}_{m i s s}\right|\right)$, yields $R_{T}$ as the slope and $\left(R_{L}+\frac{V_{T T}}{V_{L}} R_{T T}\right)$ as the intercept. Having three points on the plot lowers the uncertainties in the derived quantities, while providing a stringent test of the systematics of the experiment. For the $\theta_{p q}=16^{\circ}$ case only $2442.3 \mathrm{MeV}$ and $843.3 \mathrm{MeV}$ settings were available, and as a result $\left(R_{L}+\frac{V_{T T}}{V_{L}} R_{T T}\right)$ and $R_{T}$ had to be calculated using only two points.

The $\left(\omega, Q^{2}\right)$ phase space for the $843.3 \mathrm{MeV}$ setting was much smaller than that for the $2442.3 \mathrm{MeV}$ setting. As a result, only a small fraction of $2442.3 \mathrm{MeV}$ data could be used for the $\left(R_{L}+\frac{V_{T T}}{V_{L}} R_{T T}\right), R_{T}$ separation. For each missing energy bin, the $\left(\omega, Q^{2},\left|\vec{P}_{m i s s}\right|\right)$ phase space was matched for all kinematic settings used in the $\left(R_{L}+\frac{V_{T T}}{V_{L}} R_{T T}\right), R_{T}$ separation. The matched phase space range for $\omega$ and $Q^{2}$ was 
approximately $\pm 3 \%$ in each variable, while the matched $\left|\vec{P}_{\text {miss }}\right|$ range was $\sim \pm 15$ $\mathrm{MeV} / \mathrm{c}$.

\subsection{Systematic uncertainty}

The sources of systematic uncertainty in the measured (e,e'p) cross section are listed in table 4.1. The individual uncertainties were added in quadrature to obtain the total uncertainties given in the last two rows. The "relative uncertainty" given in the final row excludes uncertainties that could be corrected by applying the same multiplicative factor to the cross sections measured at every kinematic setting. Such "non-relative uncertainties" would affect the extracted response functions in the same manner.

\begin{tabular}{|l|l|c|}
\hline \hline Quantity & Uncertainty & \% effect on cross section \\
\hline Beam energy & $0.2 \%$ & 0.4 \\
Electron scattering angle & $0.3 \mathrm{mr}$ & 0.3 \\
Proton scattering angle & $0.3 \mathrm{mr}$ & 0.4 \\
Proton solid angle & $2.0 \%$ & 2.0 \\
Luminosity (relative) & $1.7 \%$ & 1.7 \\
Luminosity (absolute) & $4.0 \%$ & 4.0 \\
\hline Total uncertainty (absolute) & & 4.8 \\
\hline Total uncertainty (relative) & & 2.7 \\
\hline \hline
\end{tabular}

Table 4.1: Systematic errors in experiment E89-003.

Since the $H\left(e, e^{\prime}\right)$ cross section measured by the electron spectrometer was used to normalize the measured cross sections, the electron solid angle cancels out in the expression for cross section and hence does not contribute towards the systematic uncertainty.

The relative uncertainty in luminosity arises from three sources:

- statistical uncertainty in $\mathrm{H}\left(\mathrm{e}, \mathrm{e}^{\prime}\right)$ data.

- uncertainty arising from the separation of the ${ }^{16} \mathrm{O}\left(\mathrm{e}, \mathrm{e}^{\prime} \mathrm{p}\right)$ background from the $\mathrm{H}\left(\mathrm{e}, \mathrm{e}^{\prime}\right)$ elastic peak. 
- uncertainty due to the determination of the electron scattering angle which is used to evaluate the "world" $\mathrm{H}\left(\mathrm{e}, \mathrm{e}^{\prime}\right)$ cross section.

In addition to these, the absolute uncertainty in luminosity contains the absolute uncertainty of the "world" $\mathrm{H}\left(\mathrm{e}, \mathrm{e}^{\prime}\right)$ cross section, which we estimated to be $4 \%$. 


\section{Chapter 5}

\section{Results and discussion}

In this chapter the results from the experiment are presented and discussed. The results for the dip region are presented in the first part of the chapter while the latter part contains the results for the quasielastic region.

In Fig. 1-1, previously described in detail in Chapter 1, the quasielastic peak is

present as a broad bump near $\omega=\frac{Q^{2}}{2 M_{N}}$, where $M_{N}$ is the nucleon mass. $\omega=\frac{Q^{2}}{2 M_{N}}$ is the kinematics that corresponds to the elastic scattering of a free nucleon. The region between the quasielastic bump and the bump above that - the $\Delta$ resonance -, is called the dip region. 


\subsection{Dip region results}

Fig. 5-1 shows the total $\left(E_{\text {miss }},\left|\vec{P}_{\text {miss }}\right|\right)$ phase-space covered by the five HRSH settings (see table 2.3 for the dip region kinematic settings). As is evident in the figure, the momentum settings were chosen to be close enough to each other so that there was adequate overlap between them. This ensured that the radiative unfolding could be performed properly.

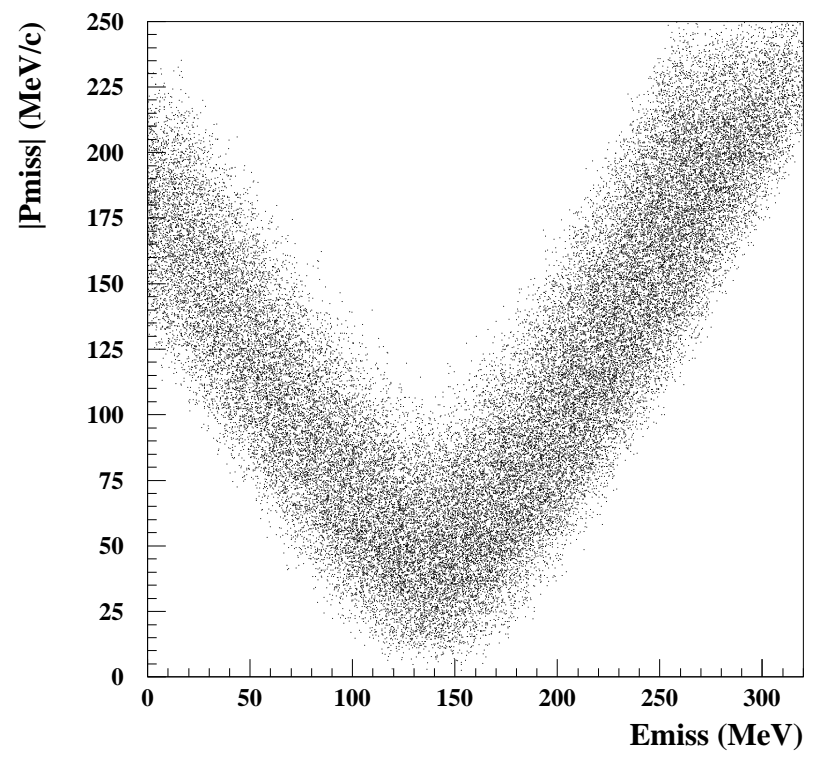

Figure 5-1: The combined $\left(E_{\text {miss }},\left|\vec{P}_{\text {miss }}\right|\right)$ space covered by the five dip region settings. 


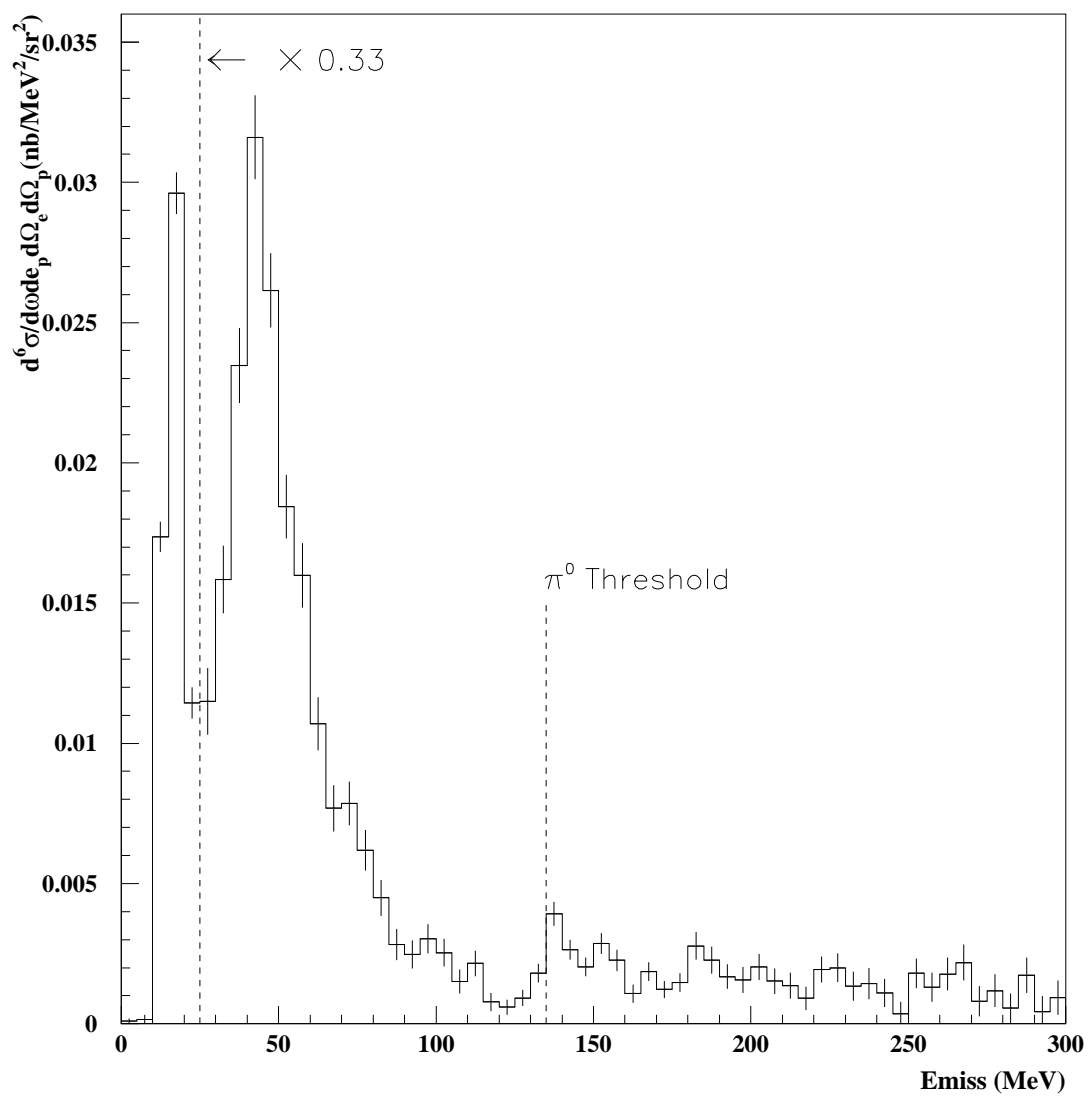

Figure 5-2: Radiatively-corrected cross section for the dip region. See Fig. 5-1 for the $\left|\vec{P}_{m i s s}\right|$ range covered at each value of $E_{\text {miss }}$. Due to binning effects, the two 1 p states at $12.1 \mathrm{MeV}$ and $18.4 \mathrm{MeV}$ show up as a single peak. The $\pi^{0}$ threshold at $134.9 \mathrm{MeV}$ is also shown.

The radiatively-corrected cross section calculated for the dip region after the gas Cherenkov cut and punch-through subtraction is shown in Fig. 5-2. This cross section shows a sharp rise at the pion production threshold $\left(E_{\text {miss }}=134.9 \mathrm{MeV}\right)$. Corresponding to this peak in the $E_{\text {miss }}$ spectrum, there is a sharp peak in the missing mass ${ }^{1}$ spectrum (Fig. 5-3) at the $\pi^{0}$ mass. Such a peak can only come from $\pi^{0}$ production from free protons because the initial momentum of bound protons smears the missing mass peak. Thus, we conclude that the increase in cross section at the pion production threshold is mainly due to $\pi^{0}$ production from $\mathrm{H}$ in the waterfall target,

\footnotetext{
${ }^{1}$ The missing mass is given by $M_{\text {miss }}=\sqrt{E_{m i s s}^{2}-P_{m i s s}^{2}}$.
} 
namely

$$
e+p \rightarrow e^{\prime}+p+\pi^{0}
$$

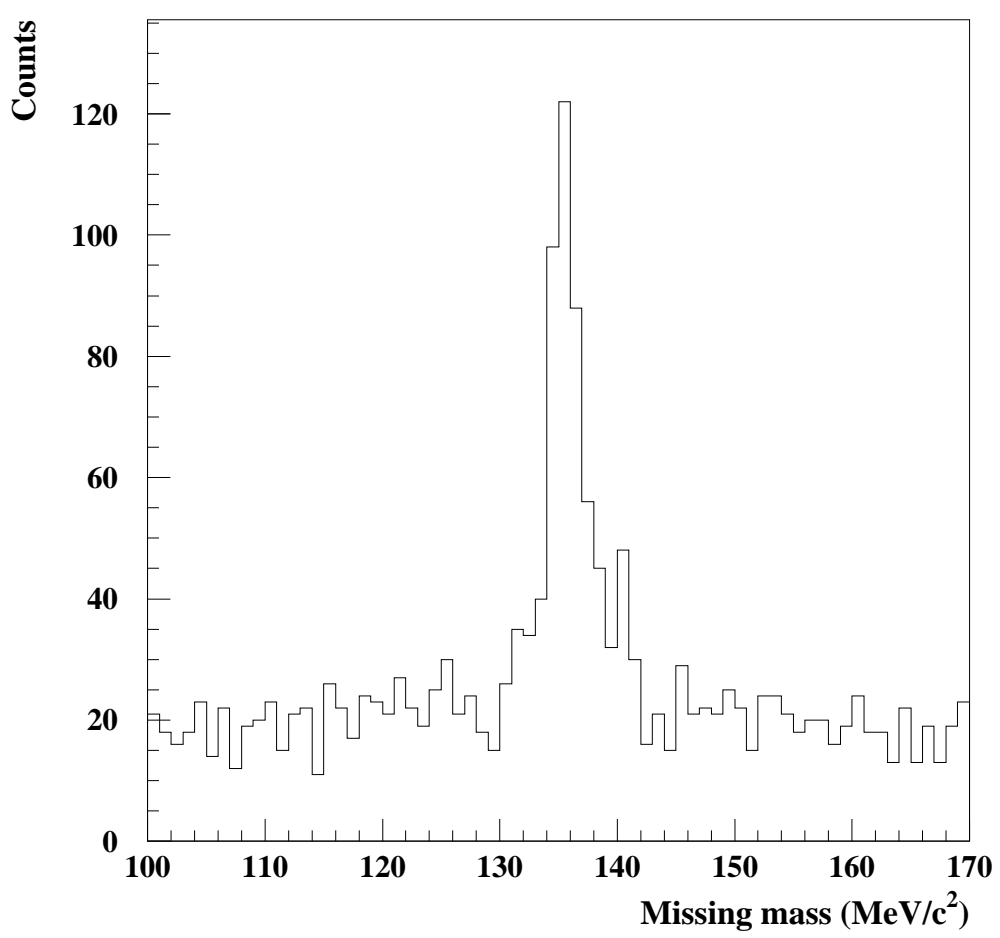

Figure 5-3: Missing mass $\left(\sqrt{E_{m i s s}^{2}-P_{m i s s}^{2}}\right)$ calculated for the dip region settings. The peak is at the $\pi^{0}$ mass.

Since the intent of this experiment is to study the ${ }^{16} \mathrm{O}\left(\mathrm{e}, \mathrm{e}^{\prime} \mathrm{p}\right)$ reaction, this $\pi^{0}$ yield from $\mathrm{H}$ is treated as contamination. A cut in the missing mass spectrum from 130 $\mathrm{MeV}$ to $140 \mathrm{MeV}$ was used for both the real data and the phase-space simulation to reject these events. 


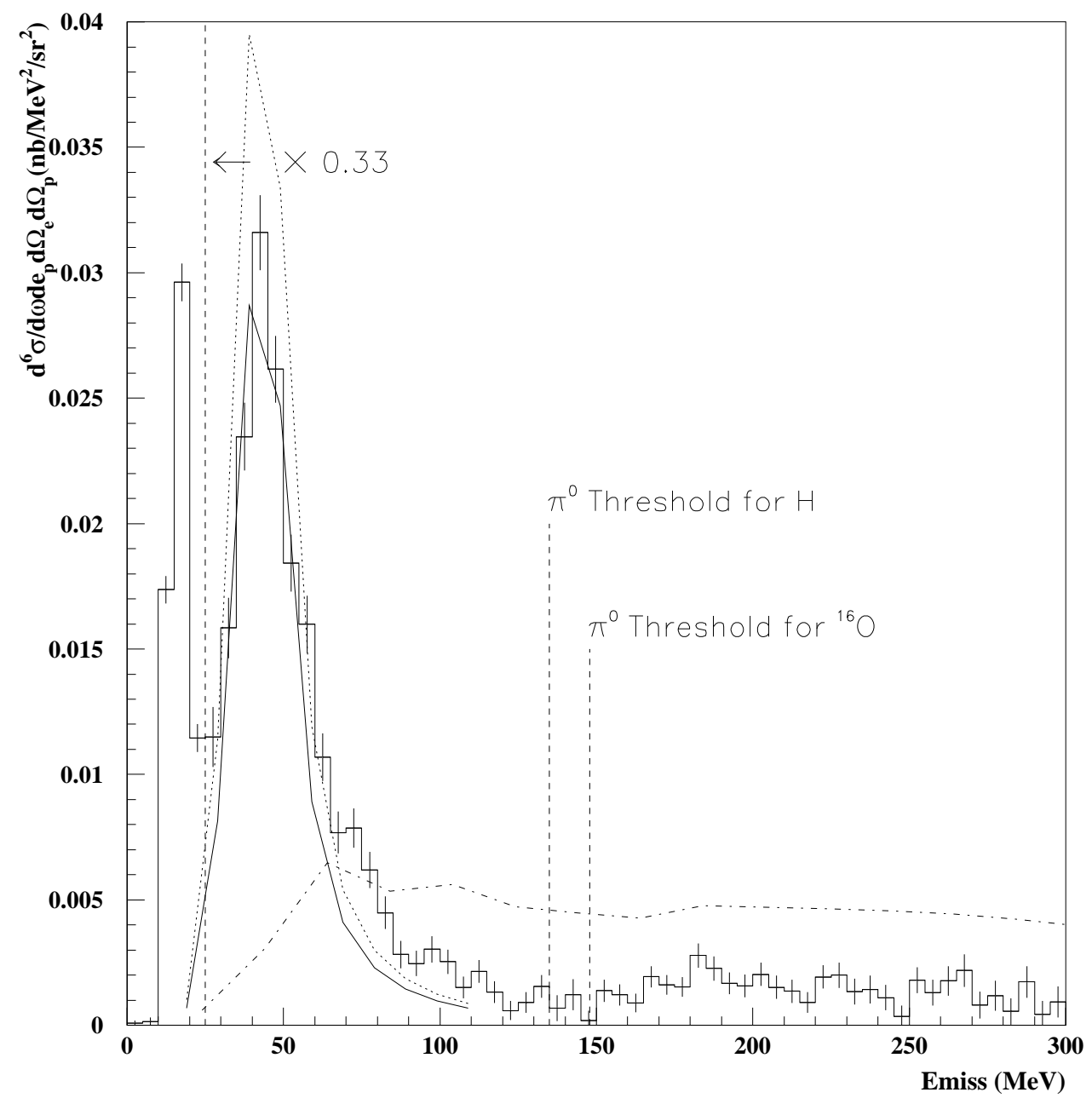

Figure 5-4: The dip region cross section with the $\mathrm{p}\left(\mathrm{e}, \mathrm{e}^{\prime} \mathrm{p} \pi^{0}\right)$ yield removed using a missing mass cut from $130 \mathrm{MeV}$ to $140 \mathrm{MeV}$. The curves present the calculations performed by Ryckebusch [43], [44], [45], [46]. The dotted line gives the 1s $1 / 2$ single-particle knockout strength calculated in the impulse approximation, while the solid curve includes contributions from meson exchange currents and $\Delta$-excitation. The dot-dashed curve gives the (e,e'pn) strength. Note that Ryckebusch calculations are preliminary

Fig. 5-4 presents the cross section with the $\mathrm{p}\left(\mathrm{e}, \mathrm{e}^{\prime} \mathrm{p} \pi^{0}\right)$ yield removed. Although removing this yield has reduced the sharp rise in cross section at the $\pi^{0}$ threshold, there remains a clear increase in cross section past this threshold.

The missing energy spectrum can be divided into several different regions, corresponding to the dominance of different reaction mechanisms. 


\subsection{1 $10.0 \mathrm{MeV}<E_{\text {miss }}<60.0 \mathrm{MeV}$ : single particle knockout of $1 \mathrm{p}_{1 / 2}$, $1 \mathrm{p}_{3 / 2}$ and $1 \mathrm{~s}_{1 / 2}$ protons}

Fig. 5-5 presents the cross sections for this missing energy range. Figures 5-4 and 55 contain theoretical curves for $1 \mathrm{~s}_{1 / 2}$ single particle knockout strength calculated by Ryckebusch et al. Shown in the figures are Impulse Approximation (IA) calculations and calculations that include two-body current contributions related to Meson Exchange Currents (MECs) and $\Delta$-creation. These calculations were performed in a self-consistent Hartree-Fock (HF), Random Phase Approximation (RPA) frame work [44], [45], [46]. The single particle wave functions were obtained by a Hartree-Fock calculation with an effective Skyrme-type interaction. For the protons the Coloumb interaction is added to the mean-field potential. The wave function for the ejectile proton and the residual nucleus is obtained from a RPA formalism that includes particle-hole and hole-particle excitations of a correlated ground state. Since the bound and continuum single particle states are eigenfunctions of the same HF mean field, orthogonality between bound and continuum states is maintained. The treatment of Final State Interactions (FSI) includes distortion by the HF mean field which is real. To account for the flux loss to complicated configurations, which is handled by complex optical potentials in the case of DWIA models, a normalization factor is used with all calculated cross sections. For all Ryckebusch $1 \mathrm{~s}_{1 / 2}$ single particle knockout calculations presented in this thesis, a normalization factor of 0.5 has been used.

The distribution of $1 \mathrm{~s}_{1 / 2}$ strength in $E_{\text {miss }}$ was obtained by using a prescription given by Jeukenne and Mahaux [77], [43], where the $1 \mathrm{~s}_{1 / 2}$ profile is modeled using a Lorentzian with an energy-dependent width $\Gamma(E)$

$$
\sigma(E)=\frac{1}{\pi} \frac{\sigma_{T} \Gamma(E)}{(E-|\epsilon|)^{2}+(1 / 4)(\Gamma(E))^{2}},
$$

where $\sigma_{T}$ is the integrated total strength and $\epsilon$ is the energy of the $1 \mathrm{~s}_{1 / 2}$ state. The width is determined using

$$
\Gamma(E)=\frac{18\left(E-S_{N}\right)^{4}}{\left(E-S_{N}\right)^{4}+13.27^{4}}(\mathrm{MeV}),
$$


which was obtained from a compilation of experimental data [77].

As the two figures indicate, the total calculation $(\mathrm{IA}+\mathrm{MEC}+\Delta)$ appears to be in good agreement with data in this $E_{\text {miss }}$ range.

Table 5.1 shows cross sections measured for $1 \mathrm{p}_{1 / 2}$ and $1 \mathrm{p}_{3 / 2}$ states compared to theoretical cross sections calculated using the program LEA [78]. LEA was developed and is maintained by Dr. J. J. Kelly. The details of the calculation are described in [79]. LEA performs a DWIA calculation and utilizes the $\vec{\Gamma}_{c c 1}$ off-shell vertex function with nucleon form factors from model 3 of Gari and Krümpelmann [80], [81]. Single nucleon overlap functions used in the calculation are Hartree-Fock wave functions based on the quasiparticle Hamiltonian model of Ma and Wambach [82], [83] which incorporates both short-range and long-range correlations. Final State Interactions (FSI) are handled by the EDAD1 potential fitted by Cooper et al. [84] The spectroscopic factors used to normalize the calculation have been determined by fitting to ${ }^{16} \mathrm{O}\left(\mathrm{e}, \mathrm{e}^{\prime} \mathrm{p}\right)$ data of Leuschner. et al [85] The spectroscopic factors thus determined for $1 \mathrm{p}_{1 / 2}$ and $1 \mathrm{p}_{3 / 2}$ states were 0.75 and 0.64 respectively.

\begin{tabular}{|l|l|l|c|}
\hline \hline State & $P_{\text {average }}$ & {$\left[\frac{d^{6} \sigma}{d \Omega_{e} d \Omega_{p} d \omega d E_{p^{\prime}}}\right]_{e x p}\left(\mathrm{nb} / \mathrm{MeV} / \mathrm{sr}^{-2}\right)$} & {$\left[\frac{d^{6} \sigma}{d \Omega_{e} d \Omega_{p} d \omega d E_{p^{\prime}}}\right]_{L E A}\left(\mathrm{nb} / \mathrm{MeV} / \mathrm{sr}^{-2}\right)$} \\
\hline \hline $1 \mathrm{p}_{1 / 2}$ & $170 \mathrm{MeV}$ & $0.35 \pm 0.012$ & 0.43 \\
\hline $1 \mathrm{p}_{3 / 2}$ & $166 \mathrm{MeV}$ & $0.67 \pm 0.017$ & 0.57 \\
\hline \hline
\end{tabular}

Table 5.1: Measured cross sections for the $1 \mathrm{p}_{1 / 2}$ and $1 \mathrm{p}_{3 / 2}$ states compared to the theoretical cross sections calculated using the program LEA [78].

The LEA calculation overestimates the $1 \mathrm{p}_{1 / 2}$ cross section by about $22 \%$ and underestimates the $1 \mathrm{p}_{3 / 2}$ cross section by about $17 \%$. In the case of quasielastic kinematics of this experiment, the measured cross sections for the $1 p_{1 / 2}$ and $1 p_{3 / 2}$ states were in good agreement with the DWIA calculations obtained using LEA [1]. It should be noted that the LEA DWIA calculation does not include MEC or $\Delta$ contributions. The discrepency between the p-shell cross sections measured in the dip region and the LEA calculation might indicate the increased importance of the $\Delta$ and MEC contributions in the dip region compared to the quasielastic region. 


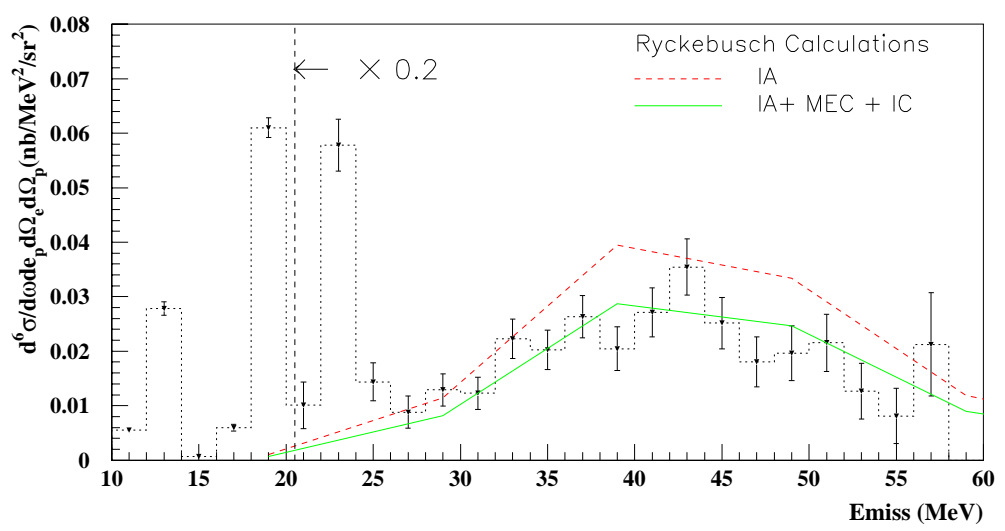

Figure 5-5: The dip region cross section for the valence states. See Fig. 5-1 for the $\left|\vec{P}_{\text {miss }}\right|$ range covered at each value of $E_{\text {miss }}$. The curves are the calculations performed by Ryckebusch. The dashed line gives the $1 \mathrm{~s}_{1 / 2}$ single particle knockout strength calculated in the impulse approximation, while the solid curve includes contributions from meson exchange currents and $\Delta$ excitation.

\subsection{MeV $<E_{m i s s}<150.0 \mathrm{MeV}$ : multi-nucleon knockout}

The two-body knockout threshold for ${ }^{16} \mathrm{O}$ is around $E_{\text {miss }} \approx 28 \mathrm{MeV}$. Thus, the multinucleon knockout is expected to contribute to the cross section in the range of 28.0 $\mathrm{MeV}<E_{\text {miss }}<150.0 \mathrm{MeV}$. Nucleon rescattering in the nucleus is also expected to contribute. Knocked-out nucleons, mainly from the valence states, interact strongly with the other nucleons in the nucleus. Due to these interactions, some nucleons are re-absorbed by the nucleus, while others re-scatter losing energy and changing the angle with which they leave the nucleus. Some of these nucleons can even re-scatter back into the spectrometer acceptance and end up in a higher $E_{m i s s}$ bin due to the energy lost in the rescattering process.

Several attempts have been made to explain the high $E_{\text {miss }}$ continuum strength observed in the Bates coincidence experiments [37], [35], [39] in terms of proton rescattering in the nucleus. Takaki [86] used a model calibrated with respect to proton scattering data and found that rescattering in the nucleus following single nucleon knockout could account for only about $8 \%$ of the cross section observed in the dip region for $E_{\text {miss }}>50 \mathrm{MeV}$ and is negligible for $E_{\text {miss }}>100 \mathrm{MeV}$. He also used a simple model to investigate two-body and three-body mechanisms at high $E_{m i s s}$, and concluded that while two-body mechanisms are important up to $E_{\text {miss }} \sim 100 \mathrm{MeV}$, 
three-body absorption becomes dominant for $100 \mathrm{MeV}<E_{\text {miss }}<200 \mathrm{MeV}$.

Weinstein [39], [40] used a cascade code developed by Bertini [87], [88] to investigate the contribution of nuclear rescattering to the high $E_{\text {miss }}$ strength. Rescattering at high $E_{\text {miss }}$ was found to go down with increasing momentum transfer $(|\vec{q}|)$, and for $|\vec{q}|=827 \mathrm{MeV}$, it accounted for less than $5 \%$ of the yield for $E_{m i s s}>50 \mathrm{MeV}$. Weinstein attributes most of the high $E_{\text {miss }}$ strength to multi-nucleon knockout, where the virtual photon is absorbed by a cluster of two or more nucleons.

Based on these calculations performed for experiments similar to the present experiment, we anticipate that nuclear rescattering would account for only a small fraction of the high $E_{m i s s}$ strength observed in this experiment.

Ryckebusch [43] calculated the (e,e'pn) contribution to the (e,e'p) cross section for the kinematics of this experiment (see Fig. 5-4). The single particle wave functions employed in this model were constructed through the HF-RPA approach described earlier. This calculation includes two-body contributions mediated by pion-exchange currents, intermediate $\Delta$ creation and central (Jastrow) short-range correlations. It should be noted that this calculation does not include any normalization factors.

The strength for the dip region appears to continue to higher $E_{m i s s}$ than indicated by the $1 \mathrm{~s}_{1 / 2}$ single particle knockout calculation. The excess strength up to about $80 \mathrm{MeV}$ in $E_{m i s s}$ is the size of the (e,e'pn) contribution predicted by Ryckebusch. However, for $E_{m i s s}>80 \mathrm{MeV}$, Ryckebusch's prediction for the (e,e'pn) contribution alone is several times larger than the total measured cross section.

\subsection{MeV $<E_{\text {miss }}<320.0 \mathrm{MeV}$ : pion production from ${ }^{16} \mathrm{O}$ in the target and multi-nucleon knockout}

The pion production contributions to the ${ }^{16} \mathrm{O}\left(\mathrm{e}, \mathrm{e}^{\prime} \mathrm{p}\right)$ cross section can be due to the following reactions:

$$
\begin{aligned}
& e+p \rightarrow e^{\prime}+\pi^{0}+p \\
& e+n \rightarrow e^{\prime}+\pi^{-}+p
\end{aligned}
$$

The scattered electron is detected in the electron spectrometer. 
To produce a $\pi$ off a nucleon bound in a nucleus and have the scattered electron and not the $\pi^{-}$detected in the electron spectrometer, the missing energy must be greater than the sum of the pion rest mass and the binding energy of the nucleon. For ${ }^{16} \mathrm{O}$ and $\pi^{0}$, this corresponds to $E_{\text {miss }} \geq 147.0 \mathrm{MeV}$. Inspection of the missing energy spectrum presented in Fig. 5-4 shows a clear increase in the cross section starting at this missing energy range. This bump is presumably due to pion production from nucleons in ${ }^{16} \mathrm{O}$.

Here we note that in a ${ }^{12} \mathrm{C}\left(\mathrm{e}, \mathrm{e}^{\prime} \mathrm{p}\right)$ experiment performed at MIT-Bates by Morrison et al. [41] with $|\vec{q}|=1 \mathrm{GeV}$ and $\omega=475 \mathrm{MeV}$ (corresponding to the center of the quasielastic peak), no increase in cross section was observed at the pion production threshold. Instead they measured a cross section slowly decreasing with $E_{\text {miss }}$ from $\sim 100 \mathrm{MeV}$ all the way up to $320 \mathrm{MeV}$. The difference between this observation for quasielastic kinematics, and the clear bump corresponding to pion production observed in the present experiment for dip kinematics may be due to the presence of the $\Delta$ resonance tail in the dip region. The tail of the $\Delta$-resonance can be expected to strongly contribute to (e, $\left.\mathrm{e}^{\prime} \mathrm{p}\right) \pi$ cross section through resonance production of pions [89] according to

$$
\begin{aligned}
& e+p \rightarrow e^{\prime}+\Delta^{+} \rightarrow e^{\prime}+\pi^{0}+p, \\
& e+n \rightarrow e^{\prime}+\Delta^{0} \rightarrow e^{\prime}+\pi^{-}+p .
\end{aligned}
$$

Thus, the behavior of the continuum cross section measured here indicate the coexistence of two reaction processes in the dip region:

- a process that is responsible for an almost flat or slowly decreasing continuum strength, as observed previously by Morrison et al. in quasielastic kinematics. The (e,e'pn) strength calculated by Ryckebusch, though several times higher than the measured strength, is also slowly decreasing for $E_{m i s s}>80 \mathrm{MeV}$. This appears to indicate the (e,e'pn) knockout could be the process one sees here, but attenuated by a factor not included in the Ryckebusch calculation.

- a process related to $\Delta$-resonant pion production, mediated by 5.6 and 5.6 


\subsection{Quasielastic region results $(|\vec{q}|=1 \mathrm{GeV}, \omega=445 \mathrm{MeV})$}

In the quasielastic region, data were acquired for both parallel and perpendicular kinematics. This section presents the results from quasielastic settings for $E_{\text {miss }}>20$ $\mathrm{MeV}$. The cross sections were measured for $20 \mathrm{MeV}<E_{\text {miss }}<120 \mathrm{MeV}$. However at high missing energies, the phase-spaces for different kinematic settings did not match. Thus, the missing energy range covered for the response function separation was 20 $\mathrm{MeV}<E_{\text {miss }}<60 \mathrm{MeV}$. The results for the $1 \mathrm{p}$ valence states from quasielastic settings are presented in [1].

For the missing energy range covered here, an important contribution the cross section is from single particle knockout from the $1 \mathrm{~s}_{1 / 2}$ shell of ${ }^{16} \mathrm{O}$. One main objective of this part of the experiment is to look for strength in this $E_{\text {miss }}$ range that cannot be explained by single particle knockout from $1 \mathrm{~s}_{1 / 2}$. Any such strength might indicate new reaction mechanisms. In order to check how much of the observed strength can be explained by $1 \mathrm{~s}_{1 / 2}$ knockout, all the results presented here were compared to the $1 \mathrm{~s}_{1 / 2}$ single particle knockout calculations by Van Orden [90], [91], Kelly [31] and Ryckebusch [44], [45], [46]. The calculations by Kelly and Ryckebusch were described in section 5.1.1.

Van Orden's calculation is a DWIA calculation performed in a relativistic framework. Both bound state and final state distorted wave functions are based on the Dirac equation: the bound state wave functions are relativistic Hartree wave functions, while microscopic Dirac optical model wave functions are used for the knockout proton. The optical potential is based on free nucleon-nucleon amplitudes and a nuclear density based on elastic electron scattering data. Effects due to meson exchange currents, isobar excitations and Coulomb distortions of the electron were not included. In this calculation, the $1 \mathrm{~s}_{1 / 2}$ shell is treated as an energy eigenstate. Since no coupling to the continuum states is included, the missing energy spectrum is a $\delta$-function spike. Further, there are no spectroscopic factors included in the calculations. They have to be determined by fitting to data.

Unlike the p-shells, the s-shell is not a localized peak in $E_{\text {miss }}$, but rather a wide bump. The strength calculated by theory should be compared to the strength inte- 
grated over this bump. In order to model the $1 \mathrm{~s}_{1 / 2}$ distribution in $E_{\text {miss }}$, a prescription given by Jeukenne and Mahaux [77] was used. Note that the same parameterization was also used by Ryckebusch et al. [43] for the calculations presented here. In this parameterization, the $1 \mathrm{~s}_{1 / 2}$ profile in $E_{\text {miss }}$ is determined by a Lorentzian with an energy dependent width $\Gamma(E)$

$$
\sigma(E)=\frac{1}{\pi} \frac{\sigma_{T} \Gamma(E)}{(E-|\epsilon|)^{2}+(1 / 4)(\Gamma(E))^{2}}
$$

where $\sigma_{T}$ is the integrated total strength and $\epsilon$ is the energy of the $1 \mathrm{~s}_{1 / 2}$ state. The width of the distribution, $\Gamma(E)$, is determined by

$$
\Gamma(E)=\frac{18\left(E-S_{N}\right)^{4}}{\left(E-S_{N}\right)^{4}+13.27^{4}}(\mathrm{MeV}),
$$

which was obtained from a compilation of experimental data [77].

In order to determine whether such a parameterization would correctly describe the $1 \mathrm{~s}_{1 / 2} E_{\text {miss }}$ profile for the present case, a setting had to be selected where the $1 \mathrm{~s}_{1 / 2}$ strength is much higher than the continuum strength. For this purpose, the $E_{0}=2442.3 \mathrm{MeV}, \theta_{p q}=-2.5^{\circ}$ setting from Experiment E89033² was selected. These data were taken under conditions similar to those in the present experiment with a nominal beam energy of $2442.3 \mathrm{MeV}$, the electron spectrometer set at an angle of $23.7^{\circ}$ and a central momentum of $2000 \mathrm{MeV}$. The values of $|\vec{q}|$ and $\omega$ were $1 \mathrm{GeV}$ and $445 \mathrm{MeV}$ respectively. For the selected setting, the missing momentum was low so that the s shell was prominent. Unlike the case of the parallel kinematic setting of the present experiment (section 5.2.1), there was no contamination from the $\mathrm{H}$ radiative tail for this setting. This is because the $\mathrm{H}$ radiative tail extends to positive values of $\theta_{p q}$, while this setting was at $\theta_{p q}=-2.5^{\circ}$.

Fig. 5-6 presents the radiatively-corrected cross section from this setting. For the whole $E_{m i s s}$ range used for this analysis, the data were integrated over the same $P_{m i s s}$ interval from $30 \mathrm{MeV} / \mathrm{c}$ to $60 \mathrm{MeV} / \mathrm{c}$. The radiatively-corrected cross section in the range $10 \mathrm{MeV}<E_{\text {miss }}<80 \mathrm{MeV}$ was modeled with Gaussians for the narrow p-

\footnotetext{
${ }^{2}$ Jefferson Lab Experiment 89033: Measurement of Recoil Polarization in the ${ }^{16} \mathrm{O}\left(\overrightarrow{\mathrm{e}}, \mathrm{e}^{\prime} \overrightarrow{\mathrm{p}}\right)$ Reaction with $2.4 \mathrm{GeV}$ Electrons [92]
} 
states, the Lorentzian shape described above for the $1 \mathrm{~s}_{1 / 2}$ state, and a linear function for the continuum background. Fig. 5-7 shows the results from the fit of this model to the measured cross section. The peak position for the $1 \mathrm{~s}_{1 / 2}$ state ( $\epsilon$ in eqn. 5.8) was determined from the fit. The isolated s shell line shape is shown in Fig. 5-8. The strength under the isolated s shell profile was integrated for $E_{\text {miss }}<80 \mathrm{MeV}$ to determine the experimentally measured total strength for the $1 s_{1 / 2}$ shell. The ratios between the total measured strength and the total $1 s_{1 / 2}$ strengths predicted by Van Orden's and Kelly's calculations were used to determine the spectroscopic factors to normalize the two calculations.

The $1 \mathrm{~s}_{1 / 2}$ spectroscopic factor thus determined for Van Orden's calculation was $88 \%$, while the spectroscopic factor determined for Kelly's calculation was $87 \%$. These $1 \mathrm{~s}_{1 / 2}$ spectroscopic factors are significantly higher than the spectroscopic factors for the $1 \mathrm{p}_{1 / 2}$ and $1 \mathrm{p}_{3 / 2}$ states determined from the present experiment [1]. Table 5.2 gives the spectroscopic factors used to normalize Van Orden's and Kelly's calculations for the three valence states.

\begin{tabular}{|c|c|c|c|}
\hline \hline Calculation & $1 \mathrm{~s}_{1 / 2}$ & $1 \mathrm{p}_{1 / 2}$ & $1 \mathrm{p}_{3 / 2}$ \\
\hline Van Orden & 0.88 & 0.61 & 0.61 \\
Kelly & 0.87 & 0.75 & 0.64 \\
\hline \hline
\end{tabular}

Table 5.2: The spectroscopic factors used to normalize Van Orden's and Kelly's calculations for the three valence states to fit the data from the present experiment. The factors for the $1 \mathrm{p}_{1 / 2}$ and $1 \mathrm{p}_{3 / 2}$ states are from reference [1].

The spectroscopic foctors determined from the present data for the $1 \mathrm{p}_{1 / 2}$ and $1 \mathrm{p}_{3 / 2}$ states are in agreement with previous experiments [1]. However, the spectroscopic factor determined here for the $1 \mathrm{~s}_{1 / 2}$ state $(0.88)$ is significantly higher than the previously reported $1 \mathrm{~s}_{1 / 2}$ spectroscopic factors which range from 0.3 to 0.6 [42], [93], [94]. The higher spectroscopic factor observed here might be due to the fact that in the present case the integration of the $1 \mathrm{~s}_{1 / 2}$ strength was performed to to $E_{\text {miss }}=80$ $\mathrm{MeV}$, whereas in the previous experiments, the integration has been performed up to lower missing energies. For example, Holtrop et al. [42] integrated over a missing energy range from $28 \mathrm{MeV}$ to $50 \mathrm{MeV}$ and obtained a spectroscopic factor of 0.41 . 
Morrison et al. [41] used an $E_{\text {miss }}$ range from $27 \mathrm{MeV}$ to $50 \mathrm{MeV}$. While they obtained a $1 \mathrm{~s}_{1 / 2}$ spectroscopic factor of 0.4 at $q=1 \mathrm{GeV}$ and $\omega=475 \mathrm{MeV}$ (center of the quasielastic peak), at $q=1 \mathrm{GeV}$ and $\omega=330 \mathrm{MeV}$ (negative side of the quasielastic peak) they obtained an unusually high spectroscopic factor of 1.0.

The integration of the present data from $E_{\text {miss }}=28 \mathrm{MeV}$ to $E_{\text {miss }}=50 \mathrm{MeV}$ results in a spectroscopic factor of 0.65 for the the $1 s_{1 / 2}$ state. However, since the Lorentzian parametrization adaptod here indicates significant $1 \mathrm{~s}_{1 / 2}$ strength from $E_{\text {miss }}=50 \mathrm{MeV}$ to $E_{\text {miss }}=80 \mathrm{MeV}$, we believe it is more appropriate to perform the integration up to $E_{\text {miss }}=80 \mathrm{MeV}$. The high missing energy tail of the Lorenzian parametrization has been attributed to correlation effects [43]. However, the measurement of $R_{L}$ by Ulmer et al. [35] and the values of $R_{L}$ extracted from the present experiment (presented later in this chapter) indicate that the strength in the apparent $1 \mathrm{~s}_{1 / 2}$ bump above $E_{\text {miss }}=50 \mathrm{MeV}$ might be due to processes other than the single particle knockout from $1 \mathrm{~s}_{1 / 2}$. 


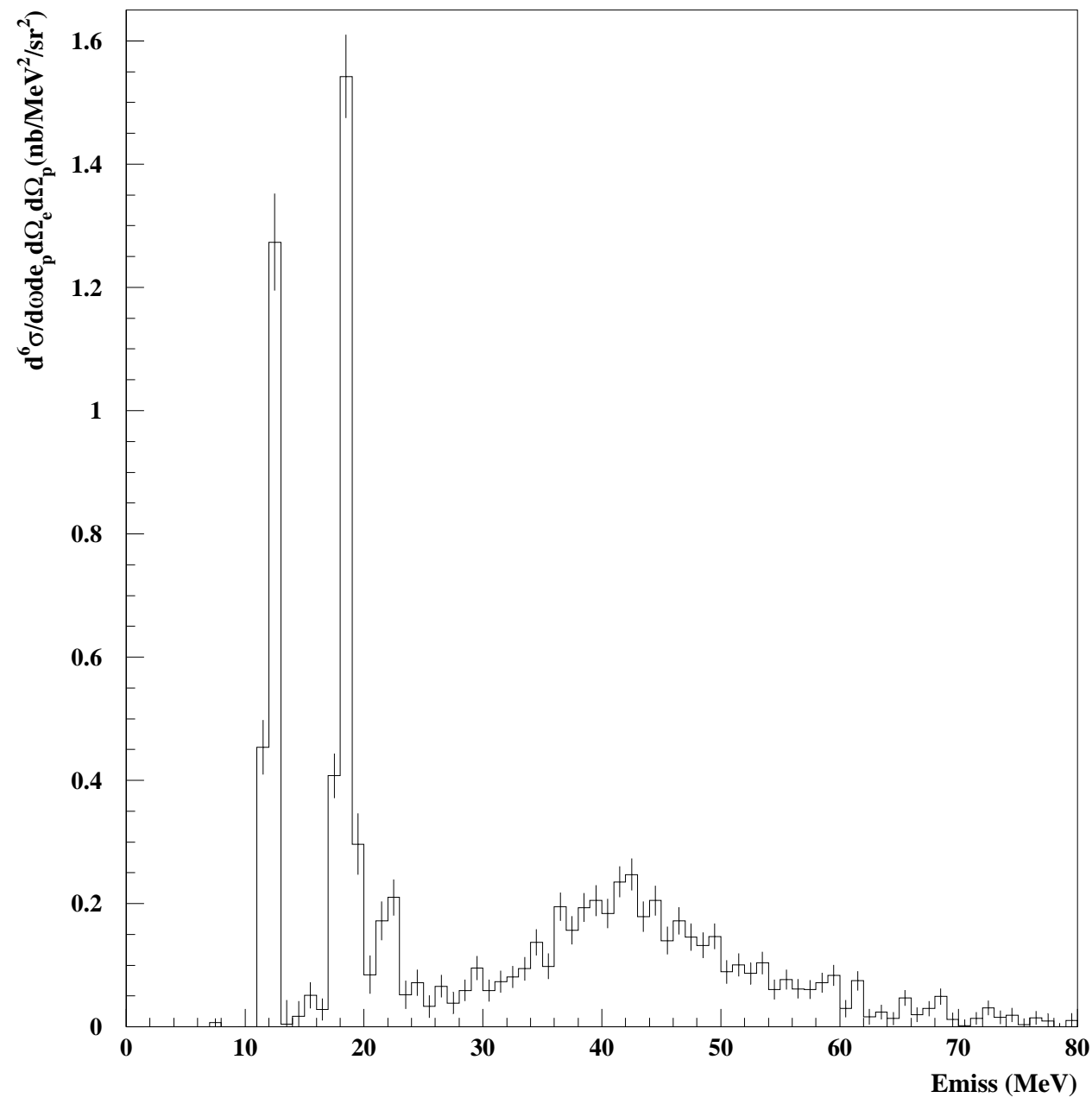

Figure 5-6: The radiatively-corrected cross section for the $E_{0}=2442.3 \mathrm{MeV}, \theta_{p q}=-2.5^{\circ}$ setting from E89033 for $30 \mathrm{MeV} / \mathrm{c}<P_{\text {miss }}<60 \mathrm{MeV} / \mathrm{c}$.

The line shape given by eqns. 5.8 and 5.9 and the spectroscopic factors determined here were used with all calculations by Van Orden and Kelly presented throughout this section. This was based on the assumption that the $E_{\text {miss }}$ profile of the $1 \mathrm{~s}_{1 / 2}$ state and the spectroscopic factor are independent of missing momentum. 


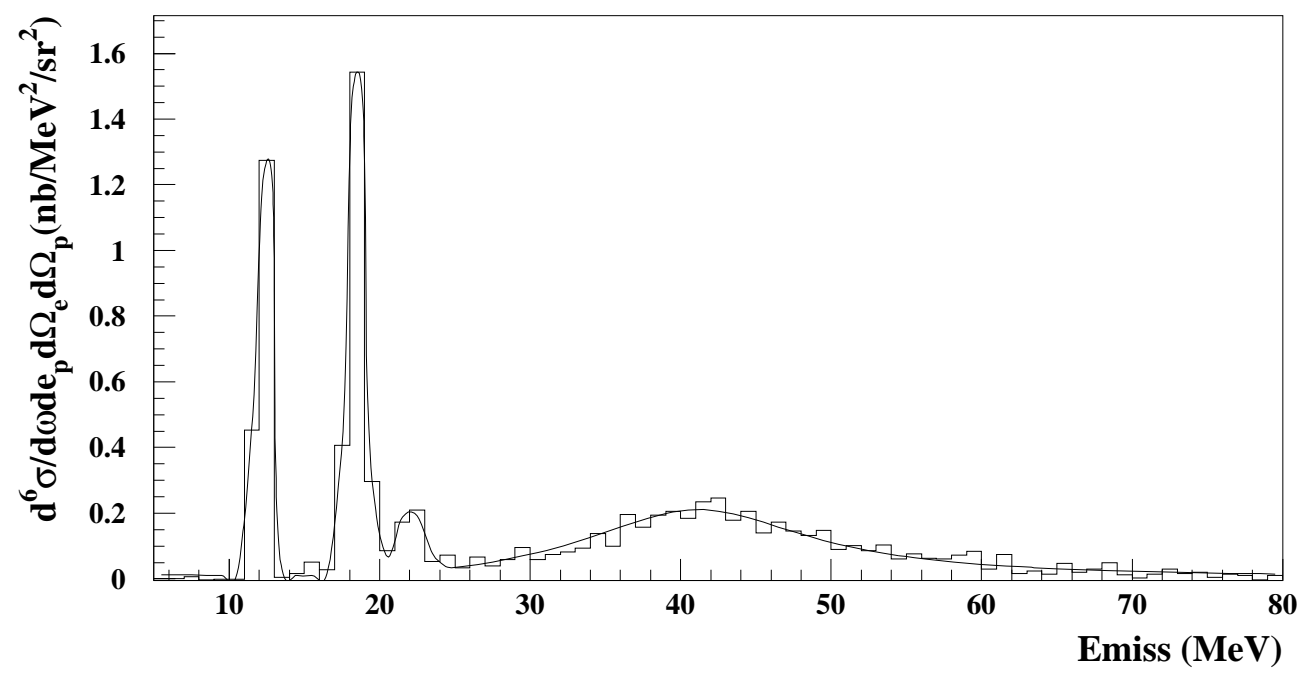

Figure 5-7: Line fits to the $E_{m i s s}$ spectrum from the $E_{0}=2442.3 \mathrm{MeV}, \theta_{p q}=-2.5^{\circ}$ setting. A fourteen parameter fit with Gaussians for the narrow p states, the Lorentzian shape described in the text for the $1 \mathrm{~s}_{1 / 2}$ state, and a linear function for the continuum background was used. The plot shows the total line fit superimposed on data.

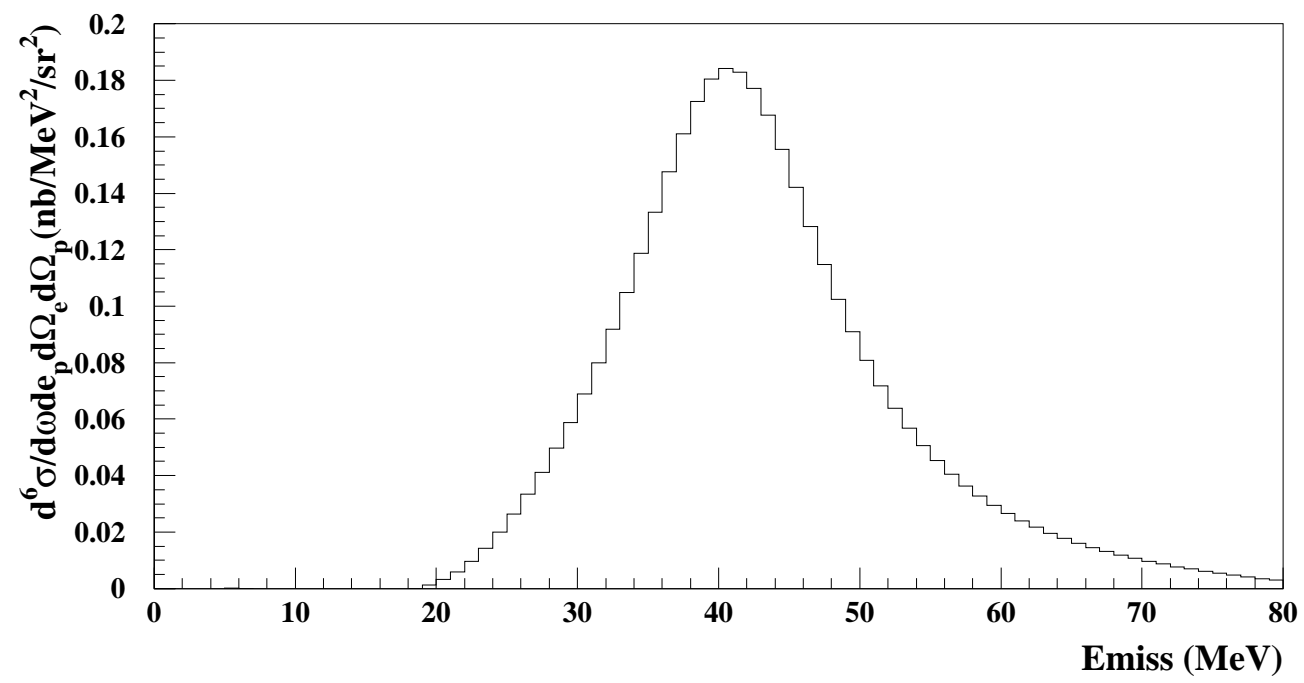

Figure 5-8: The isolated s shell line shape based on the energy dependent Lorentzian parameterization.

\subsubsection{Quasi-parallel kinematics (Quasielastic kinematics: $|\vec{q}|=1 \mathrm{GeV}$, $\omega=445 \mathrm{MeV}$ )}

Data were acquired in parallel kinematics at beam energies of $843.3 \mathrm{MeV}, 1642.5 \mathrm{MeV}$ and 2442.5 MeV during this experiment. Most of the true coincidence events in the 
parallel kinematics settings were $\mathrm{H}\left(\mathrm{e}, \mathrm{e}^{\prime} \mathrm{p}\right)$ events from the waterfall target. Fig. 5-9-a presents the $\left(E_{\text {miss }}, P_{\text {miss }}\right)$ distribution for the $E_{0}=843.3 \mathrm{MeV}$ setting. The dark cluster of events at $\left(E_{\text {miss }}=0 \mathrm{MeV}, P_{\text {miss }}=0 \mathrm{MeV}\right)$ is the $\mathrm{H}\left(\mathrm{e}, \mathrm{e}^{\prime} \mathrm{p}\right)$ peak. In the absence of radiation, the $\mathrm{H}\left(\mathrm{e}, \mathrm{e}^{\prime} \mathrm{p}\right)$ reaction is completely determined by the detection of the scattered electron and the proton. Thus, for the $\mathrm{H}\left(\mathrm{e}, \mathrm{e}^{\prime} \mathrm{p}\right)$ peak $E_{\text {miss }}=0 \mathrm{MeV}$ and $P_{\text {miss }}=0 \mathrm{MeV}$. The radiation from the incoming and the scattered electrons change $E_{m i s s}$ and $P_{m i s s}$ for $\mathrm{H}\left(\mathrm{e}, \mathrm{e}^{\prime} \mathrm{p}\right)$. However, $E_{\text {miss }}$ and $P_{\text {miss }}$ are related to each other by the equation

$$
M_{m i s s}^{2}=E_{m i s s}^{2}-P_{m i s s}^{2},
$$

where $M_{\text {miss }}$ is the missing mass for the reaction. Since the only "missing particle" in the $\mathrm{H}\left(\mathrm{e}, \mathrm{e}^{\prime} \mathrm{p}\right)$ reaction (including radiation) is the massless photon, ideally $E_{\text {miss }}=$ $P_{m i s s}$ for the $\mathrm{H}\left(\mathrm{e}, \mathrm{e}^{\prime} \mathrm{p}\right)$ radiative tail. In reality, $E_{m i s s}$ and $P_{\text {miss }}$ can only be calculated to the accuracy limits set by angular and momentum resolutions of the spectrometers. Therefore, for the $\mathrm{H}\left(\mathrm{e}, \mathrm{e}^{\prime} \mathrm{p}\right)$ radiative tail calculated from real data, $E_{\text {miss }}$ is equal to $P_{\text {miss }}$ only within the resolution allowed by the spectrometers. The diagonal line extending from the $\mathrm{H}\left(\mathrm{e}, \mathrm{e}^{\prime} \mathrm{p}\right)$ peak (at $E_{\text {miss }}=0$ and $P_{\text {miss }}=0$ ) is the radiative tail from $\mathrm{H}\left(\mathrm{e}, \mathrm{e}^{\prime} \mathrm{p}\right)$, while the band of events extending parallel to the $P_{\text {miss }}$ axis is due to widening of the $\mathrm{H}\left(\mathrm{e}, \mathrm{e}^{\prime} \mathrm{p}\right)$ peak in $P_{\text {miss }}$ as a result of the angular resolution of the spectrometer. 


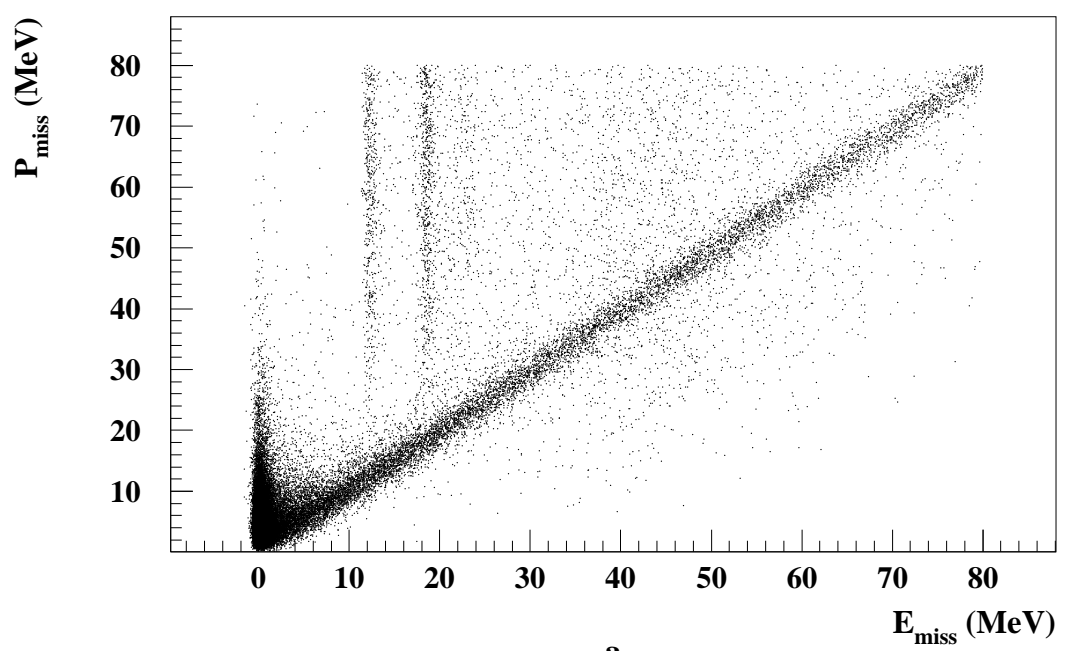

a

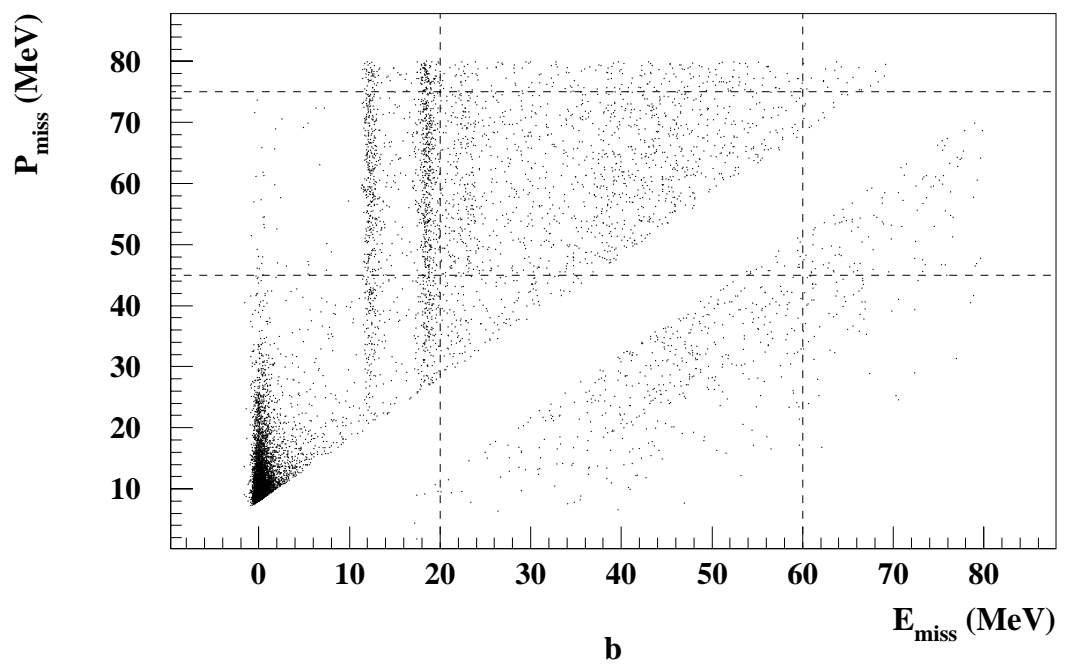

Figure 5-9: The $\left(E_{m i s s}, P_{m i s s}\right)$ distribution sampled for quasi-parallel kinematics. The top plot presents the uncut distribution while the bottom plot was obtained after the $\mathrm{H}\left(\mathrm{e}, \mathrm{e}^{\prime} \mathrm{p}\right)$ radiative tail was removed by a software cut. The rectangular area indicated by dashed lines in the bottom plot was the $\left(E_{m i s s}, P_{m i s s}\right)$ phase space used to obtain the results presented in this thesis. The plots are from $E_{0}=2442.3 \mathrm{MeV}$ setting.

The strength of the $\mathrm{H}\left(\mathrm{e}, \mathrm{e}^{\prime} \mathrm{p}\right)$ peak was so large compared to the ${ }^{16} \mathrm{O}\left(\mathrm{e}, \mathrm{e}^{\prime} \mathrm{p}\right)$ strength that the radiative tail from $\mathrm{H}$ overwhelms the ${ }^{16} \mathrm{O}$ strength at all $E_{\text {miss }}$ values sampled in this experiment. Due to the magnitude of the $\mathrm{H}$ radiative tail, we believe that it is not meaningful to remove the tail by radiative corrections. Instead, a software cut to remove all events with $\left|E_{\text {miss }}-P_{\text {miss }}\right|<10 \mathrm{MeV}$ was used to get rid of the $\mathrm{H}$ 
tail. Fig. 5-9.(b) presents the $\left(E_{m i s s}, P_{m i s s}\right)$ distribution with this cut applied. In addition to this cut, a $P_{\text {miss }}$ cut $\left(45 \mathrm{MeV}<P_{\text {miss }}<75 \mathrm{MeV}\right)$ was used to provide a well defined phase-space volume as a function of $E_{\text {miss }}$.

It should be noted that due to the above cuts, the data used for this analysis are not in strictly parallel kinematics. The $P_{\text {miss }}$ cut described above removes all the events inside a sphere located at the tip of $\vec{q}$ as illustrated in Fig. 5-10. As a result, a large fraction of the $\vec{P}_{\text {miss }}$ vectors that are parallel to $\vec{q}$ are removed. However, since $\vec{P}_{m i s s}$ vectors are symmetrically distributed around $\vec{q}$, we believe that the data sample used here approaches the conditions of parallel kinematics in so far as the interference response functions, dependent on $\phi$ (the azimuthal angle around $\vec{q}$ ), average out to zero. Due to this problem we call this setting "quasi-parallel" kinematics.

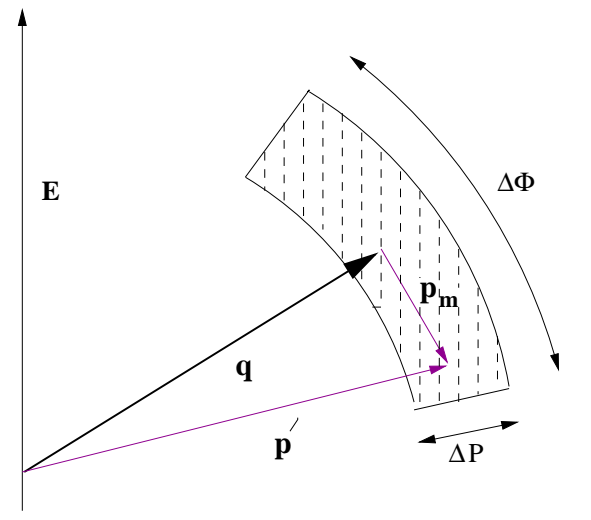

(a)

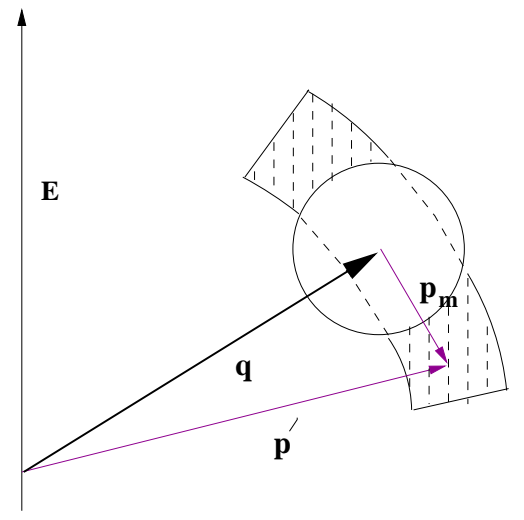

(b)

Figure 5-10: The shaded area in (a) shows the momentum $(\mathrm{P})$-inplane angle $(\phi)$ acceptance of the hadron spectrometer for the case of parallel kinematics without any cuts. For this setting $|\vec{q}|=1000$ $\mathrm{MeV}, \Delta \mathrm{P}=70 \mathrm{MeV}$, and $\Delta \phi \approx 3^{\circ}$. A cut of $P_{\text {miss }}<45$ removes a sphere centered around the tip of $\vec{q}$ resulting in the exclusion of the $P_{\text {miss }}$ vectors parallel to $\vec{q}$ as shown in (b).

Fig. 5-11 presents the radiatively-corrected cross sections for the three beam energy settings. The bump centered at $41 \mathrm{MeV}$ corresponds to $1 \mathrm{~s}_{1 / 2}$ single particle knockout. The method outlined in section 4.4.2 was used for the $R_{L}, R_{T}$ separation. Since the interference response functions are zero for parallel kinematics, a plot of $\sigma / K \sigma_{M} V_{L}$ vs. $V_{T} / V_{L}$ gives $R_{T}$ as the slope and $R_{L}$ as the intercept. Fig. 5-12 shows the $\sigma / K \sigma_{M} V_{L}$ vs. $V_{T} / V_{L}$ plots for all $E_{\text {miss }}$ bins used for this analysis. A chi-squared fit to each plot provides $R_{L}, R_{T}$ and their uncertainties at a given $E_{m i s s}$ bin. For most of the 
bins, the three points lie on a straight line within the uncertainty. Given the validity of the Rosenbluth separation, this indicates that the systematics of the experiment are under control. It would have been desirable if the intermediate point was close to the middle. However, the available accelerator beam energies dictated the $V_{T} / V_{L}$ values used for this experiment.

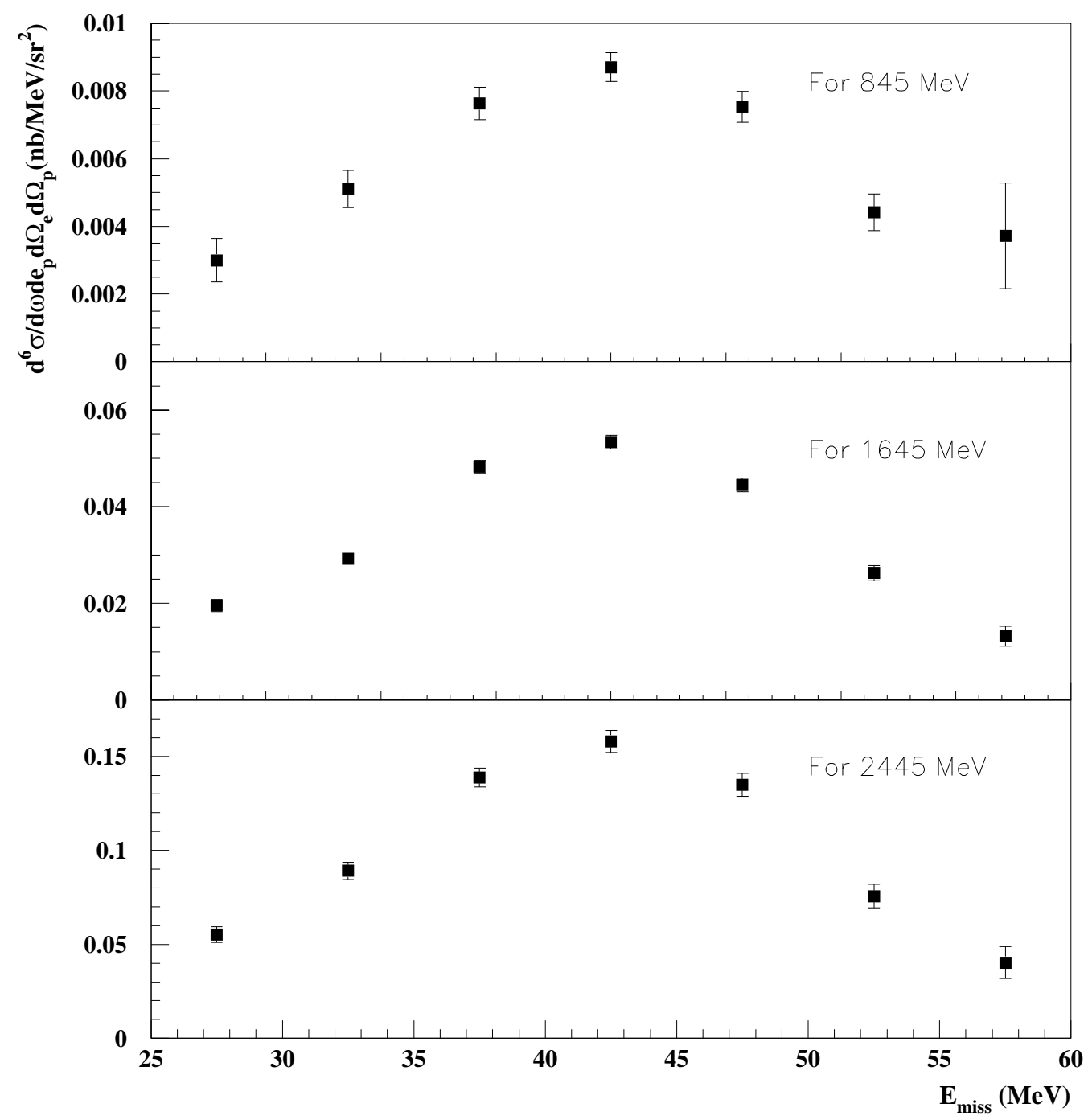

Figure 5-11: The radiatively-corrected cross sections for the three quasi-parallel settings. The range of $P_{\text {miss }}$ sampled at each $E_{\text {miss }}$ bin is different as shown in Fig. 5-9.b. 


\subsubsection{Separated response functions}

The cross section spectra at the three beam energies clearly show the $1 \mathrm{~s}_{1 / 2}$ single proton knockout peak from approximately $E_{\text {miss }}=25 \mathrm{MeV}$ to $E_{\text {miss }}=50 \mathrm{MeV}$. Above $50 \mathrm{MeV}$ the cross section decreases faster for the two higher energy settings than for the $E_{0}=843.3 \mathrm{MeV}$ setting. This is a clear sign that above $50 \mathrm{MeV}, R_{T}$ is stronger than $R_{L}$. The separated response function plots presented in Fig. 5-13 show that that this indeed is the case. Both $R_{T}$ and $R_{L}$ exhibit bumps corresponding to $1 \mathrm{~s}_{1 / 2}$ knockout. While $R_{L}$ goes to zero above this bump, $R_{T}$ remains strong.

The separated response functions are compared to the following theoretical calculations for the $1 \mathrm{~s}_{1 / 2}$ single particle knockout (the details of the calculations were given in previous sections):

1. Relativistic DWIA calculation by Van Orden [90], [91] (with the spectroscopic factor determined by fitting to the $\theta_{p q}=-2.5^{\circ}$ data as described previously).

2. Hartree-Fock, Random Phase Approximation (HF-RPA) calculation by Ryckebusch [43], [44], [45], [46] performed in the Impulse Approximation (IA).

3. HF-RPA calculation in IA including Meson Exchange Currents (MEC) and $\Delta$ excitations (Ryckebusch).

Ryckebusch's calculations contain a spectroscopic factor of 0.5 determined by fitting the calculation including MEC and $\Delta$-excitations to the longitudinal response function reported by Ulmer et al. [35], [36] for the ${ }^{12} \mathrm{C}\left(\mathrm{e}, \mathrm{e}^{\prime} \mathrm{p}\right)$ reaction. The $1 \mathrm{~s}_{1 / 2}$ $E_{m i s s}$ profile used with all three calculations has been determined using the energy dependent Lorentzian parameterization given in eqns. 5.8 and 5.9.

The curves indicate that the measured response functions are in fair agreement with relativistic DWIA and total HF-RPA calculations. The HF-RPA calculation in the IA without $\mathrm{MEC}+\Delta$ appears to overestimate data. The inclusion of $\mathrm{MEC}+\Delta$ terms in the HF-RPA calculation seems to bring it closer to data (and to the relativistic DWIA calculation).

Also shown in the figure is the difference between the longitudinal and transverse 
spectral functions, $S_{T}-S_{L}$. $S_{T}$ and $S_{L}$ were calculated as follows

$$
\begin{aligned}
& S_{L}=\frac{\sigma_{M} V_{\mathrm{L}} R_{\mathrm{L}}}{\sigma_{L}^{e p}}, \\
& S_{T}=\frac{\sigma_{M} V_{\mathrm{T}} R_{\mathrm{T}}}{\sigma_{T}^{e p}},
\end{aligned}
$$

where $\sigma_{L}^{e p}$ and $\sigma_{T}^{e p}$ are the longitudinal and transverse parts of the off-shell ep cross section obtained using deForest's CC1 prescription. 


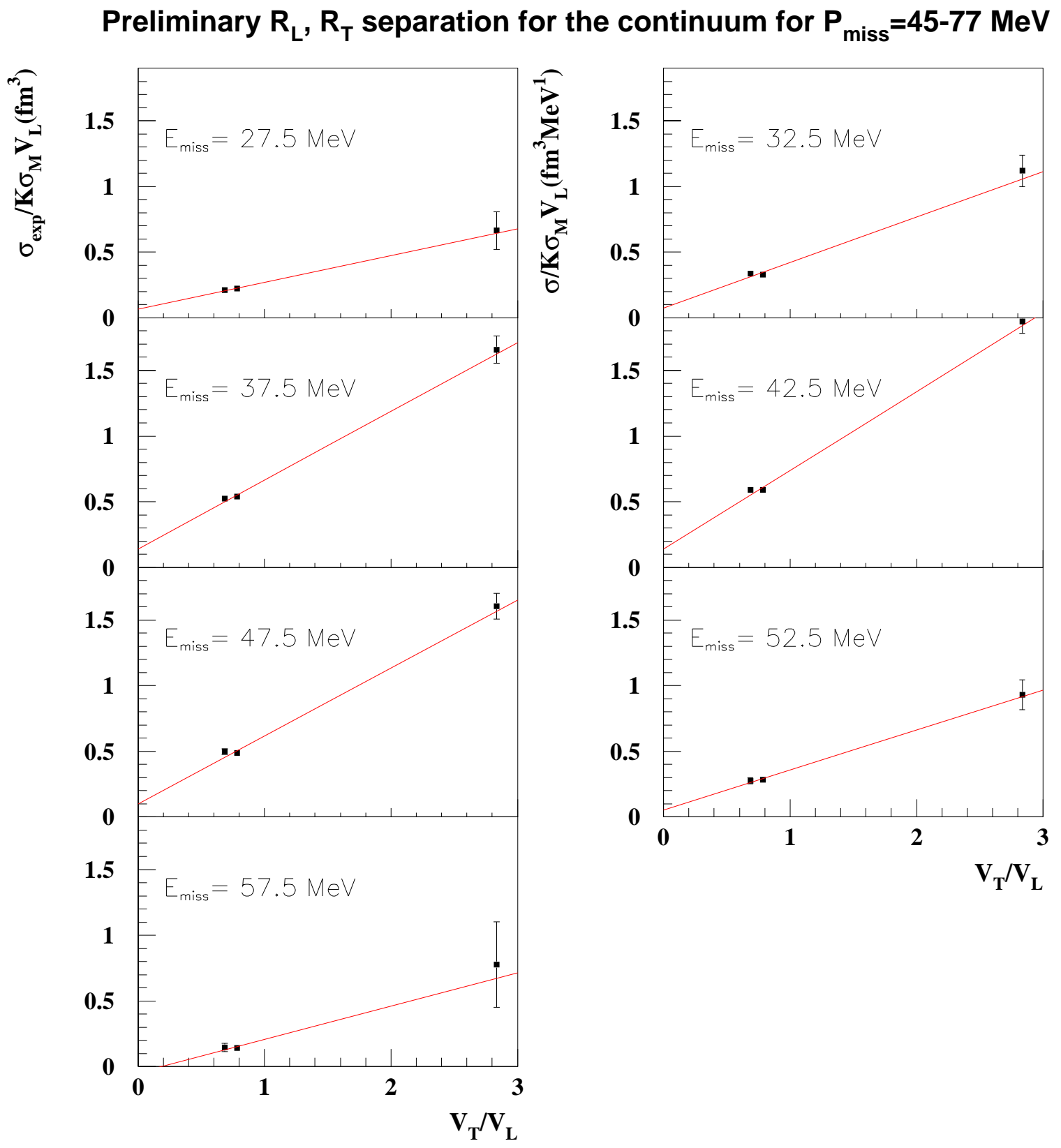

Figure 5-12: The $\sigma / K \sigma_{M} V_{L}$ vs. $V_{T} / V_{L}$ plots for quasi-parallel kinematics for all $E_{m i s s}$ bins. 


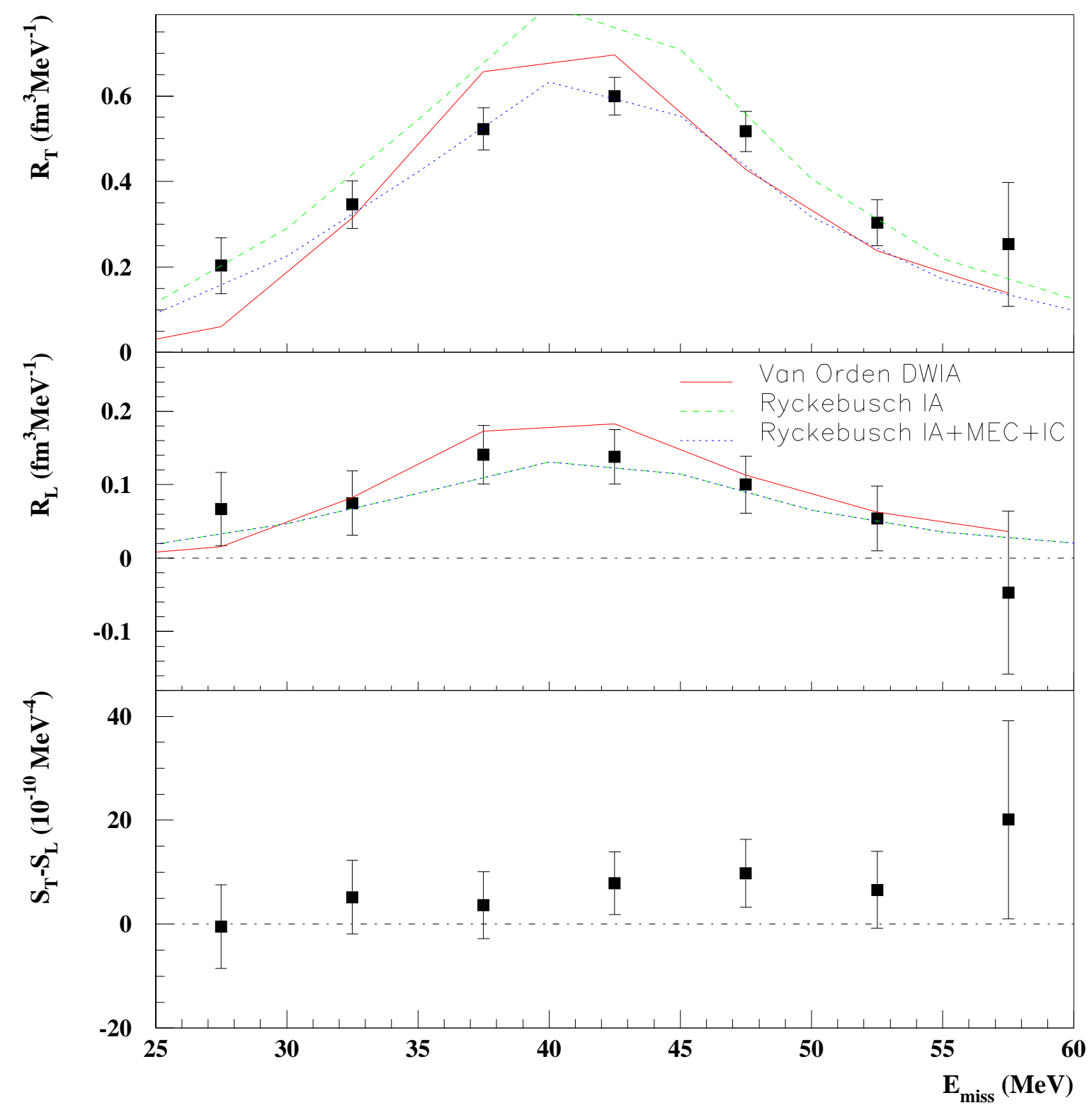

Figure 5-13: The separated response functions for quasi-parallel kinematics for $45 \mathrm{MeV} / \mathrm{c}<p_{\text {miss }}<$ $75 \mathrm{MeV} / \mathrm{c}$. The solid curves give the $1 \mathrm{~s}_{1 / 2}$ strength calculated by Van Orden (folded with the spectroscopic factor and $E_{m i s s}$ profile determined in section 5.2). The dashed curve shows the Ryckebusch Impulse Approximation (IA) calculation for $1 \mathrm{~s}_{1 / 2}$ while the dotted curve shows the total IA calculation including Meson Exchange Currents (MEC) and $\Delta$ contributions. Note that for the case of $R_{L}$ the two calculations by Ryckebusch coincide since MEC and the $\Delta$ have no effect on the longitudinal response. 
$S_{T}$ and $S_{L}$ are expected to be equal for quasifree knockout of protons exhibiting free on-shell behavior [36]. The $S_{T}-S_{L}$ plot shows that $S_{T}$ and $S_{L}$ are essentially equal up to $E_{\text {miss }}=30 \mathrm{MeV}$, and the difference between them shows a tendency to increase slowly after that. Within the statistical error of the present measurement, this is consistent with the results reported by Ulmer et al. [35] for ${ }^{12} \mathrm{C}$ (Fig. 1-7), and is also consistent with $R_{T} / R_{L}$ enhancement observed at NIKHEF-K for ${ }^{6} \mathrm{Li}$ and ${ }^{12} \mathrm{C}$ [95], [96], [97].

The (e,e'pn) contribution to the $\left(e, e^{\prime} p\right)$ cross section for the kinematics of this experiment was calculated by Ryckebusch [43]. The calculated response functions are compared to results in Fig. 5-14. As the plots indicate, the transverse response dominates the (e,e'pn) contribution. However, the magnitude of the (e,e'pn) contribution from the calculation is several orders of magnitude smaller than the single particle knockout strength. 


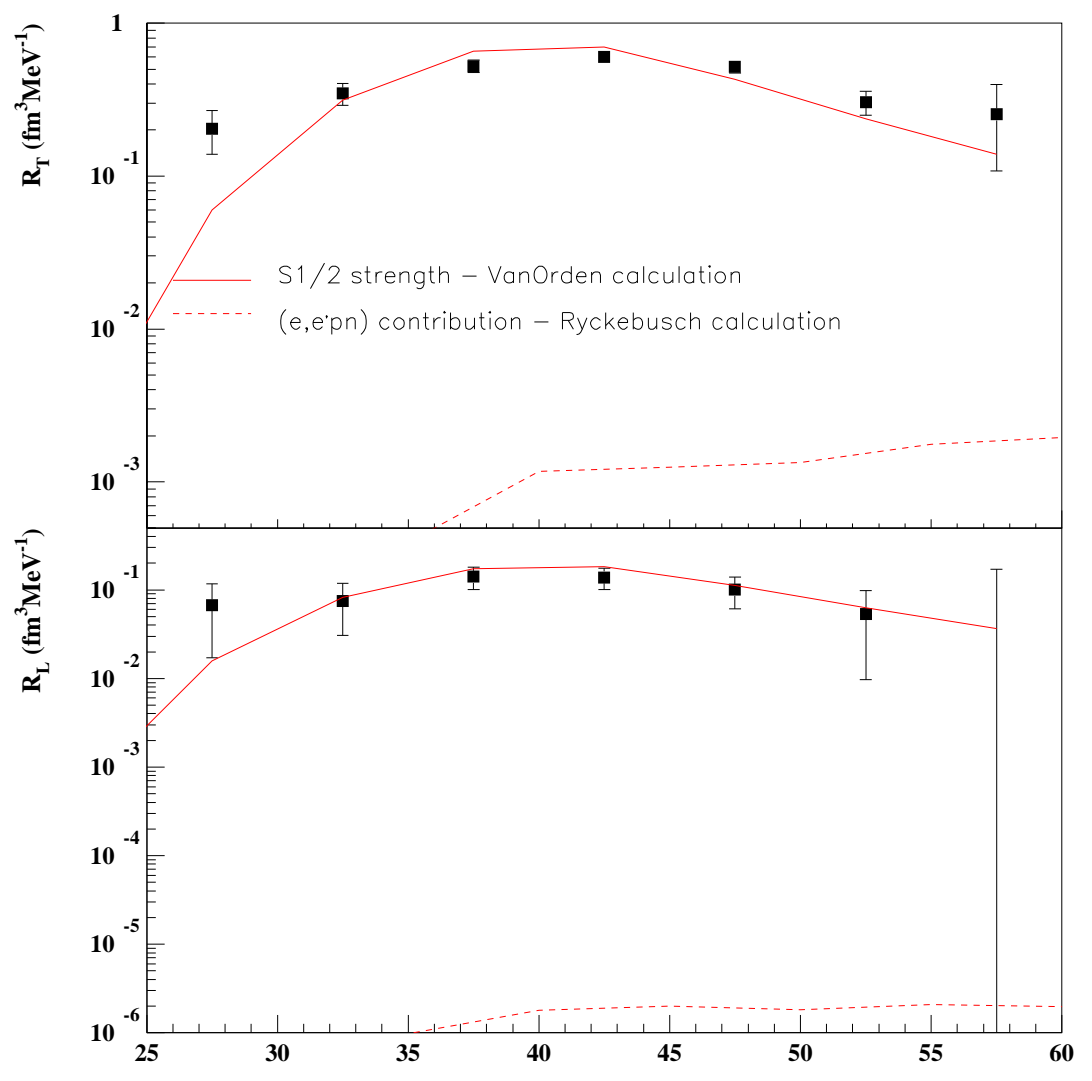

Figure 5-14: Comparison of the calculated response functions for quasi-parallel kinematics (presented earlier in Fig. 5-13 on a linear scale) to the $1 \mathrm{~s}_{1 / 2}$ strength calculated by Van Orden (folded with the spectroscopic factor and $E_{m i s s}$ distribution determined in section 5.2), and to the (e,e'pn) strength calculated by Ryckebusch. The Ryckebusch calculation is preliminary.

\subsubsection{Perpendicular kinematic settings (Quasielastic kinematics: $|\vec{q}|=1$ $\mathrm{GeV}, \omega=445 \mathrm{MeV}$ )}

Data were acquired in perpendicular kinematics at $\left|\theta_{p q}\right|$ values of $8^{\circ}, 16^{\circ}$ and $20^{\circ}$, corresponding to central recoil momenta of approximately $145 \mathrm{MeV} / \mathrm{c}, 280 \mathrm{MeV} / \mathrm{c}$ and $350 \mathrm{MeV} / \mathrm{c}$ respectively. For each setting, the cross section was measured for $0 \mathrm{MeV}$ $<E_{\text {miss }}<120 \mathrm{MeV}$. For the $\left|\theta_{p q}\right|=20^{\circ}$ settings, lack of statistics and inadequate phase-space matching made the separation of response functions impossible. This section presents separated response functions, $R_{L}+\frac{V_{T T}}{V_{L}} R_{T T}, R_{T}$, and $R_{L T}$, for the other two $\left|\theta_{p q}\right|$ settings as well as the measured cross sections for all settings. 
The procedures outlined in sections 4.4 .1 and 4.4 .2 were used for the response function separation.

\subsubsection{1 $\left|\theta_{p q}\right|=8^{\circ}$ setting $\left(P_{\text {miss }}^{\text {average }} \approx 145 \mathrm{MeV} / \mathbf{c}\right)$}

Fig. 5-15 presents the radiatively-corrected cross sections for the $E_{0}=2442.3 \mathrm{MeV}$, $\left|\theta_{p q}\right|=8^{\circ}$ settings along with the $\left(E_{\text {miss }}, P_{\text {miss }}\right)$ phase space simulation for the two settings. As the phase space plot indicates the two settings sample very different ranges in $P_{m i s s}$ at high $E_{m i s s}$. Thus, the difference in the continuum cross sections for the two settings does not represent true left-right asymmetry. Only the phase space matched $E_{\text {miss }}$ range $\left(E_{\text {miss }}<60 \mathrm{MeV}\right)$ can be used for the calculation of the left-right asymmetry and the response functions.

For both settings, the DWIA calculation seems to underestimate the data. In the $E_{\text {miss }}$ region beyond the $1 \mathrm{~s}_{1 / 2}$ bump, the $\theta_{p q}=+8^{\circ}$ setting appears to contain more excess continuum strength than the $\theta_{p q}=-8^{\circ}$ setting.

Figs. 5-16 and 5-17 present the radiatively-corrected cross sections for the $\left|\theta_{p q}\right|=8^{\circ}$ settings measured at $E_{0}=1642.5 \mathrm{MeV}$ and $E_{0}=843.3 \mathrm{MeV}$ respectively. These cross sections also show behavior similar to that of the $E_{0}=2442.3 \mathrm{MeV}$ cross sections. 


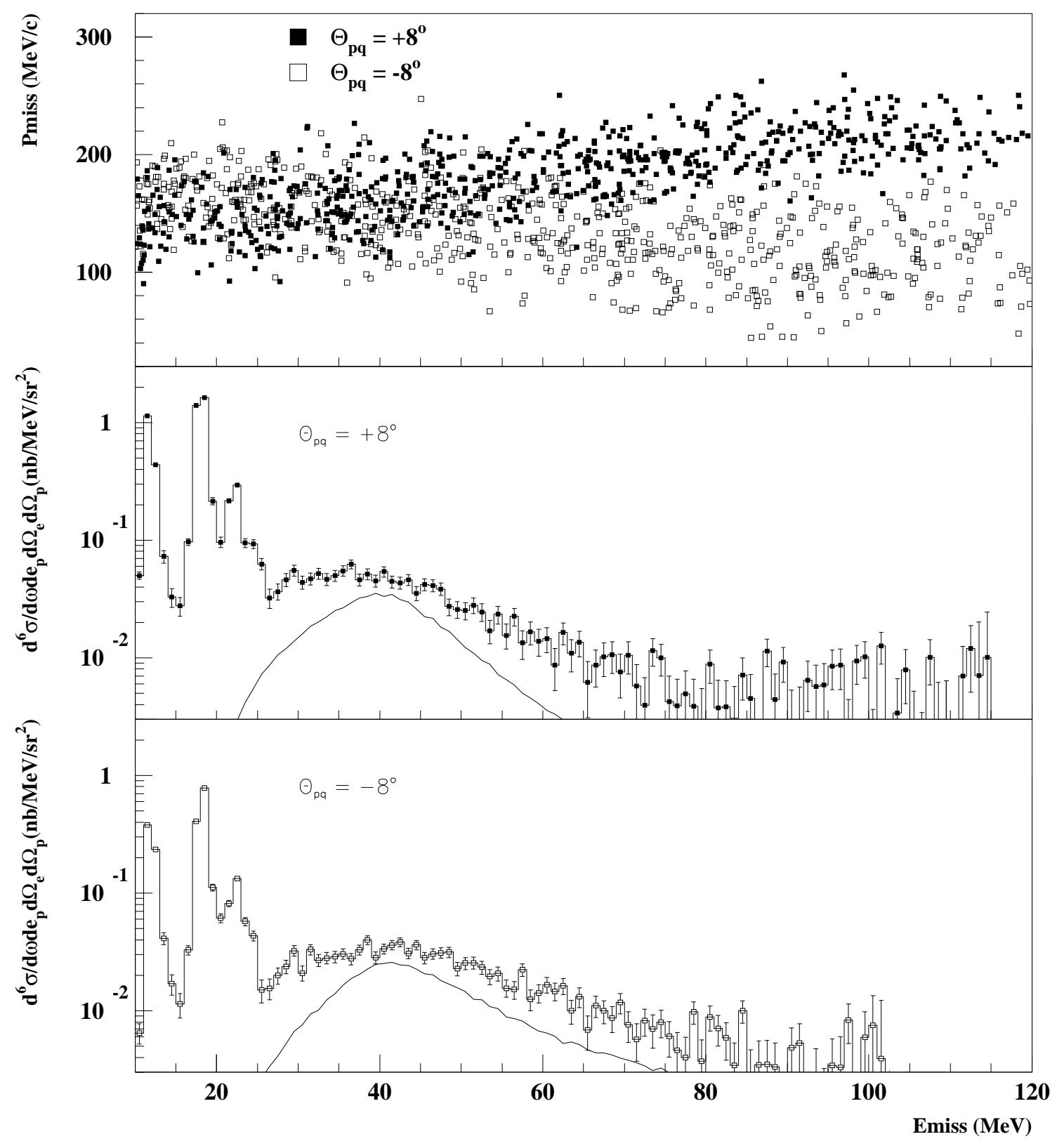

Figure 5-15: radiatively-corrected cross sections for the $E_{0}=2442.3 \mathrm{MeV},\left|\theta_{p q}\right|=8^{\circ}$ kinematic settings. The top plot gives the $\left(E_{m i s s}, P_{m i s s}\right)$ phase space for the two settings. The solid curves indicate the calculation by Van Orden for single particle knockout from the $1 \mathrm{~s}_{1 / 2}$ state. (The spectroscopic factors and the missing energy distributions were obtained as described previously.) 


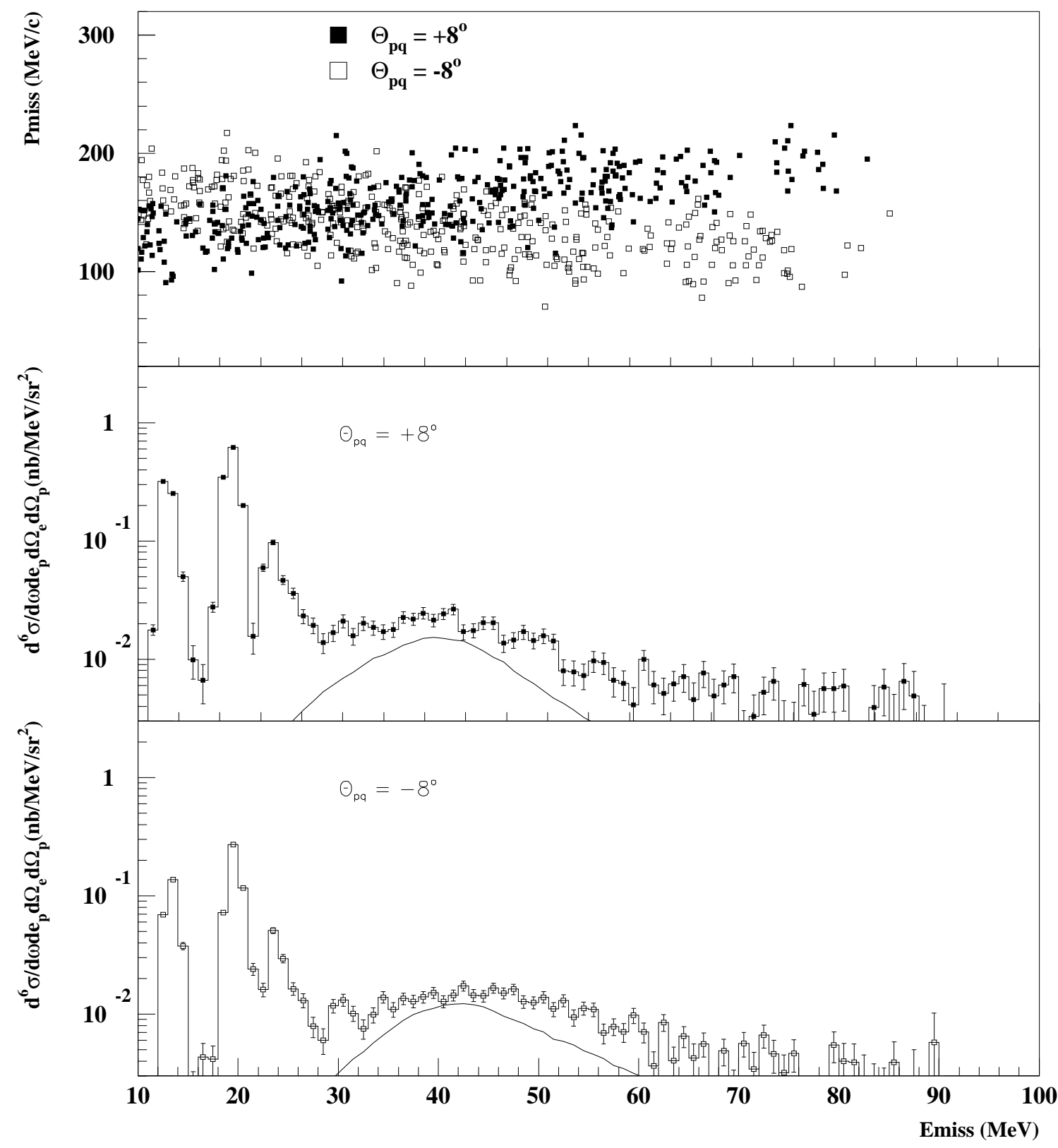

Figure 5-16: radiatively-corrected cross sections for the $E_{0}=1642.5 \mathrm{MeV},\left|\theta_{p q}\right|=8^{\circ}$ kinematic settings. The top plot gives the $\left(E_{m i s s}, P_{m i s s}\right)$ phase space for the two settings. The solid curves indicate the calculation by Van Orden for single particle knockout from the $1 \mathrm{~s}_{1 / 2}$ state. 


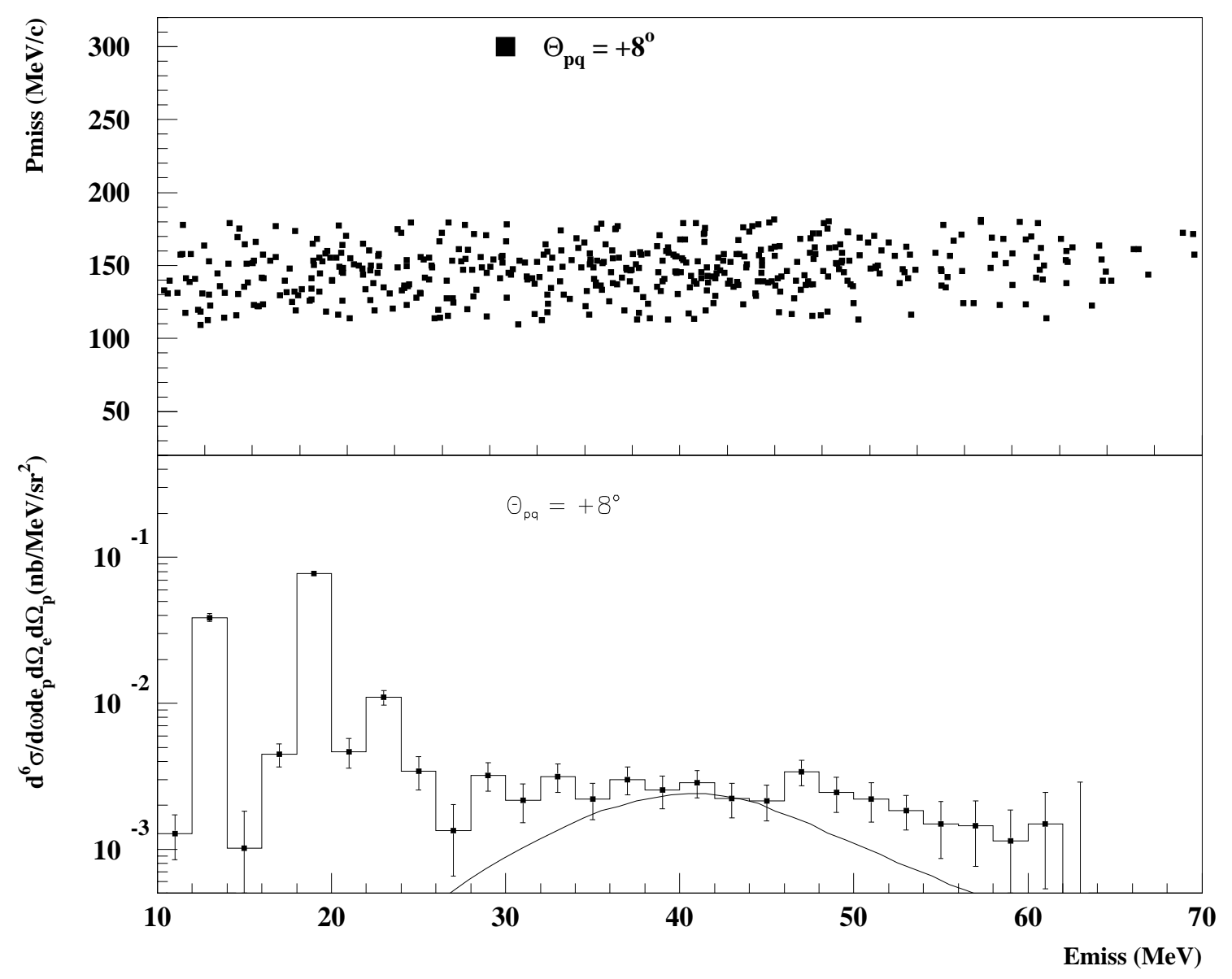

Figure 5-17: radiatively-corrected cross sections for the $E_{0}=843.3 \mathrm{MeV},\left|\theta_{p q}\right|=+8^{\circ}$ kinematic setting. The top plot gives the $\left(E_{\text {miss }}, P_{\text {miss }}\right)$ phase space for this setting. The solid curve indicate the calculation by Van Orden for single particle knockout from the $1 \mathrm{~s}_{1 / 2}$ state.

\subsubsection{2 $R_{L T}$ Separation}

For the $1642.5 \mathrm{MeV}$ and $2442.3 \mathrm{MeV}$ settings of this experiment, data were acquired on either side of $\vec{q}$ at $\theta_{p q}=+8^{\circ}$ and $\theta_{p q}=-8^{\circ}$. At each of the two beam energies, the data from the two sides were combined to calculate $R_{L T}$ for each $5 \mathrm{MeV} E_{\text {miss }}$ bin from $25 \mathrm{MeV}$ to $60 \mathrm{MeV}$. Since the response functions are independent of the beam energy, the $R_{L T}$ values calculated at the two energies should be equal. As Fig. 5-18 indicates $R_{L T}$ calculated at the two beam energies agrees well within statistical error. This is another indication that the systematic errors for the experiment are smaller than the statistical errors. It also shows that the impulse approximation assumptions used in the description of the (e,e'p) reaction are accurate. The overall $R_{L T}$ at each 
$E_{\text {miss }}$ was obtained by averaging the two $R_{L T}$ values for that bin. The averaged $R_{L T}$ values are shown in Fig. 5-20.

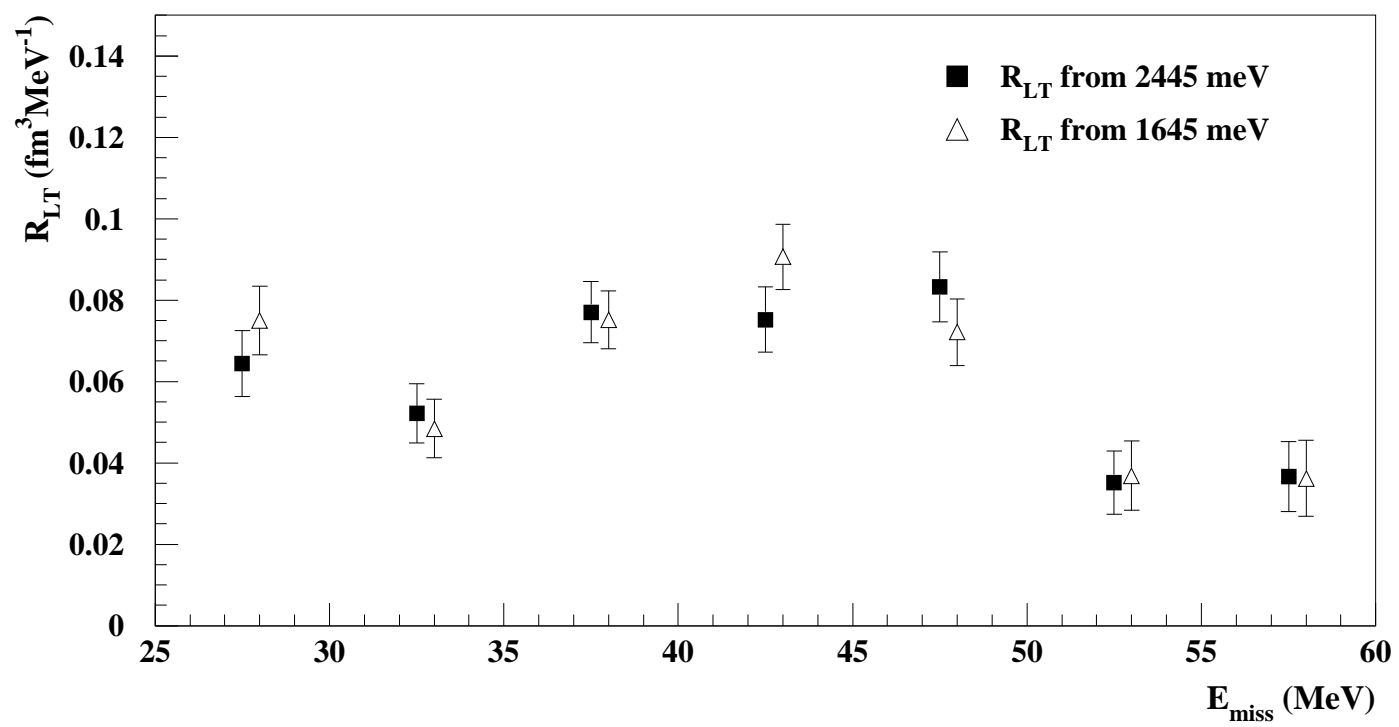

Figure 5-18: $R_{L T}$ calculated from $E_{0}=2442.3 \mathrm{MeV}$ data and from $E_{0}=1642.5 \mathrm{MeV}$ data $\left(P_{\text {miss }}^{\text {average }} \approx 145 \mathrm{MeV} / \mathrm{c}\right)$.

\subsubsection{Separation of $R_{L}+\frac{V_{T T}}{V_{L}} R_{T T}$ and $R_{T}$}

Data were acquired at all three beam energies for $\left|\theta_{p q}\right|=8^{\circ}$, rendering three points on each $\Sigma / V_{L}$ vs. $V_{T} / V_{L}$ plot ( $\Sigma$ was defined in section 4.4.2) for the separation of $R_{L}+\frac{V_{T T}}{V_{L}} R_{T T}$ and $R_{T}$. Fig. 5-19 shows the $\Sigma / V_{L}$ vs. $V_{T} / V_{L}$ plots for the missing energy bins sampled at this angular setting. Fig. 5-20 presents the separated response functions.

The separated response functions are compared to DWIA (relativistic and nonrelativistic) and HF-RPA calculations (with and without $\mathrm{MEC}+\Delta$ ) described previously. All four calculations underestimate the measured $R_{L T}$ over the whole range of $E_{m i s s}$. As in the case of quasi-parallel kinematics, the inclusion of MEC $+\Delta$ in the HF-RPA calculation seems to bring it closer to the data (and to the relativistic DWIA calculation). For $E_{\text {miss }}<35 \mathrm{MeV}$, all response functions indicate more strength than predicted by the calculations. For $E_{\text {miss }}>50 \mathrm{MeV}, R_{L}+\frac{V_{T T}}{V_{L}} R_{T T}$ appears to go to zero, while in agreement with the calculations within the statistical uncertainty. Since $\frac{V_{T T}}{V_{L}} R_{T T}$ is about $7 \%$ of $R_{L}$, this behavior can be attributed mainly to $R_{L}$. Thus, $R_{L}$ 
measured in perpendicular kinematics appears to go to zero for $E_{\text {miss }}>50 \mathrm{MeV} . R_{T}$, on the other hand, appears to retain strength above $50 \mathrm{MeV}$. This is in agreement with the high $E_{\text {miss }}$ behavior of $R_{L}$ and $R_{T}$ observed in parallel kinematics during the present experiment and also at MIT-Bates by Ulmer et al. [35], [36]
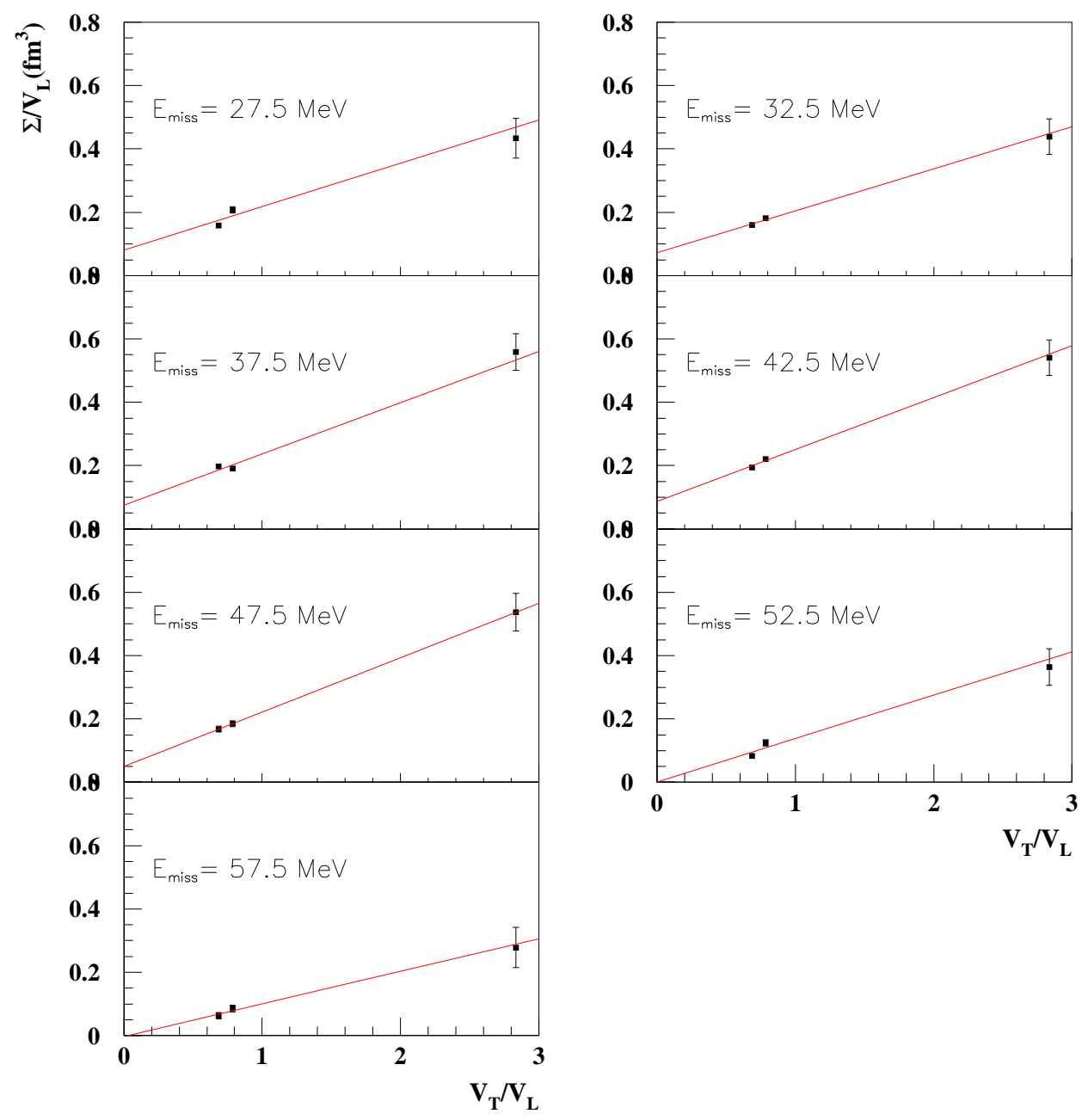

Figure 5-19: $\Sigma / V_{L}$ vs. $V_{T} / V_{L}$ plots ( $\Sigma$ was defined in section 4.4.2) used for the separation of $R_{L}+\frac{V_{T T}}{V_{L}} R_{T T}$ and $R_{T}$ at $\left|\theta_{p q}\right|=8^{\circ}\left(P_{\text {miss }}^{\text {average }} \approx 145 \mathrm{MeV} / \mathrm{c}\right)$. 


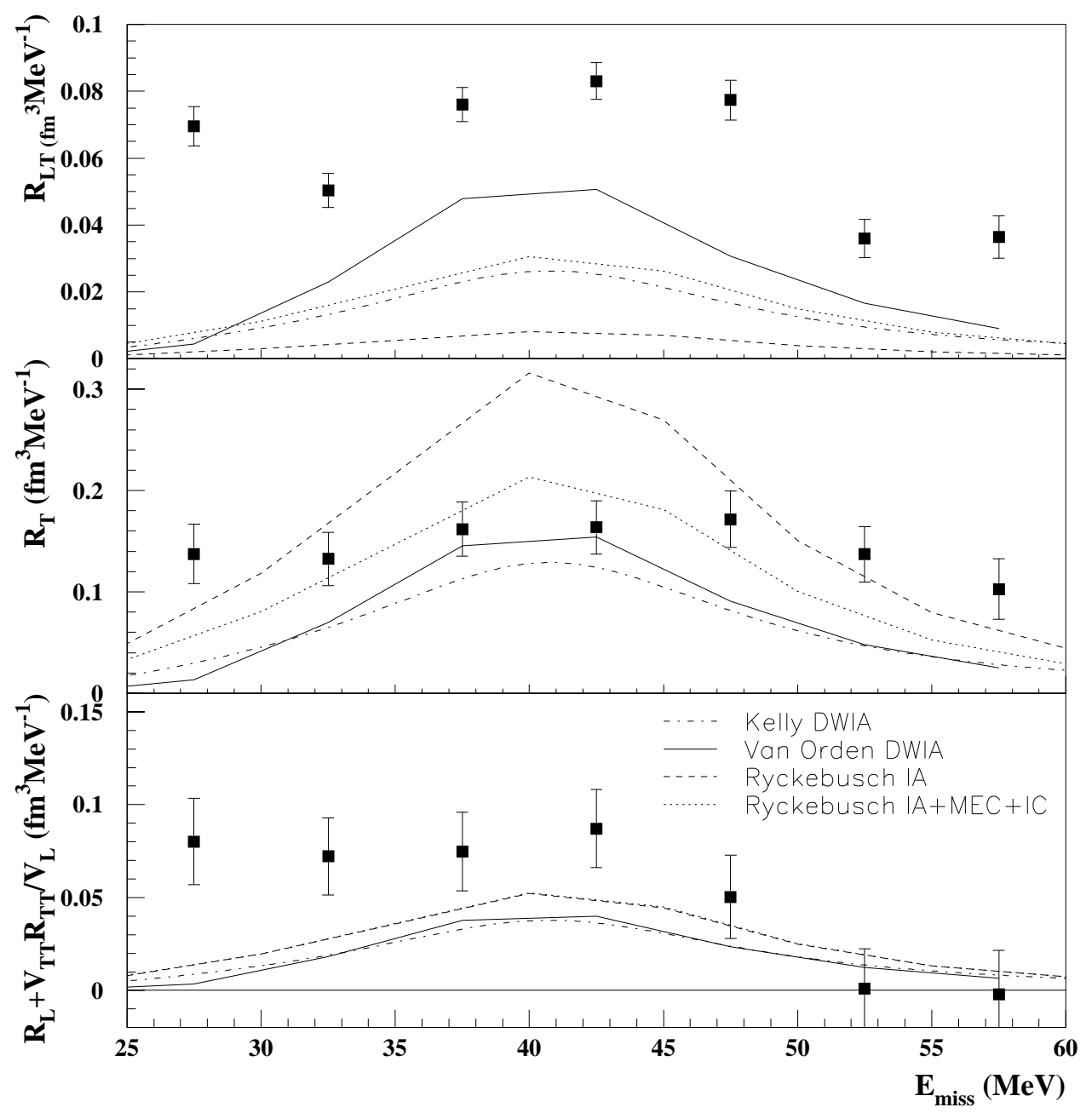

Figure 5-20: Separated response functions for the $\left|\theta_{p q}\right|=8^{\circ}$ setting $\left(P_{\text {miss }}^{\text {average }} \approx 145 \mathrm{MeV} / \mathrm{c}\right)$. Plots show (from top to bottom) $R_{L T}, R_{T}$, and $R_{L}+\frac{V_{T T}}{V_{L}} R_{T T}$. The solid curves indicate the calculations by Van Orden for single particle knockout from the $1 \mathrm{~s}_{1 / 2}$ state. The dot dashed curves indicate the calculations by Kelly for the $1 \mathrm{~s}_{1 / 2}$ state. The dashed curve shows the Ryckebusch Impulse Approximation (IA) calculation for $1 \mathrm{~s}_{1 / 2}$ while the dotted curve shows the total IA calculation including Meson Exchange Currents (MEC) and $\Delta$ contributions.Note that for the case of $R_{L}+$ $\frac{V_{T T}}{V_{L}} R_{T T}$, the two calculations by Ryckebusch are indistinguishable. 


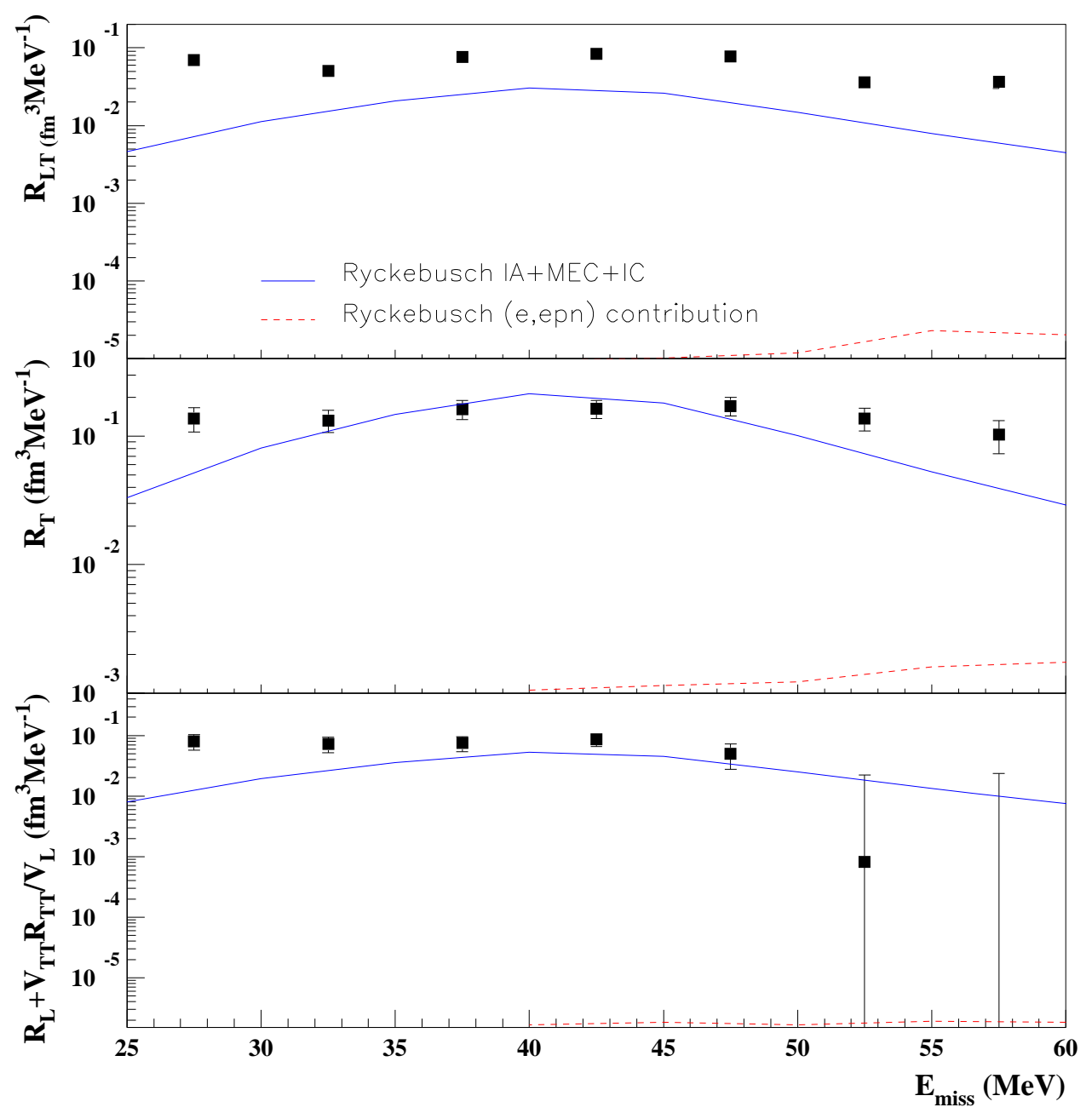

Figure 5-21: Comparison of the calculated response functions for $\left|\theta_{p q}\right|=8^{\circ}$ kinematics (presented in Fig. 5-20 on a linear scale) to the $1 \mathrm{~s}_{1 / 2}$ strength calculated by Ryckebusch in the Impulse Approximation including MECs and ICs and to the (e,e'pn) strength calculated by Ryckebusch. The calculations are preliminary.

It should be noted that the observed behavior of $R_{L T}$ for $E_{\text {miss }}>50 \mathrm{MeV}$ appears to be in disagreement with the $R_{L}$ behavior. Since $R_{L T}$ results from the interference of the longitudinal and transverse components of the virtual photon polarization, a non zero $R_{L T}$ requires a non zero $R_{L}$. However ( $R_{L}$, for the case of quasi-parallel kinematics) and $R_{L}+\frac{V_{T T}}{V_{L}} R_{T T}$ (for the case of perpendicular kinematics) seem to go to zero for $E_{\text {miss }}>50 \mathrm{MeV}$ while the measured $R_{L T}$ is clearly non zero for this range of $E_{m i s s}$. Considering the significant statistical uncertainties associated with $R_{L}$ (and 
with $R_{L}+\frac{V_{T T}}{V_{L}} R_{T T}$ ), it may in fact be possible that the value of $R_{L}$ is sufficiently non zero for $50 \mathrm{MeV}<E_{\text {miss }}<60 \mathrm{MeV}$ to be in agreement with $R_{L T}$. Further, for the case of perpendicular kinematics, the DWIA calculation by Van Orden indicates that $R_{T T}$ is negative. If this is the case then the measured value of $R_{L}$ is small but non zero for this range of $E_{m i s s}$. A non zero longitudinal component, although small, may be able to generate the observed $R_{L T}$ through interference with the unusually high transverse component at this range of $E_{\text {miss }}$.

The $\left(\mathrm{e}, \mathrm{e}^{\prime} \mathrm{pn}\right)$ contribution to the $\left(\mathrm{e}, \mathrm{e}^{\prime} \mathrm{p}\right)$ single particle knockout calculated by Ryckebusch [43] is compared to the measured response functions in Fig. 5-14. As in the case of quasi-parallel kinematics, the magnitude of the $\left(\mathrm{e}, \mathrm{e}^{\prime} \mathrm{pn}\right)$ contribution from the calculation is several orders of magnitude smaller than the single particle knockout strength and is not sufficient to explain the excess $R_{L T}$ and $R_{T}$ strength at high $E_{\text {miss }}$.

\subsubsection{4 $\left|\theta_{p q}\right|=16^{\circ}$ setting $\left(P_{\text {miss }}^{\text {average }} \approx \mathbf{2 8 0 . 0} \mathrm{MeV} / \mathbf{c}\right)$}

Fig. 5-22 presents the radiatively-corrected cross section for the $E_{0}=2442.3 \mathrm{MeV}$, $\left|\theta_{p q}\right|=16^{\circ}$ kinematic setting. The relative strength above $25 \mathrm{MeV}$ compared to the strength in the two p states shows a significant increase from the lower $P_{\text {miss }}$ settings. Unlike in the case of lower $P_{m i s s}$ settings, no clear increase in cross section is observed at the location of the $1 \mathrm{~s}_{1 / 2}$ shell. This indicates that much of the strength at $E_{\text {miss }}>$ $25 \mathrm{MeV}$ measured at this high $P_{\text {miss }}$ might be due to sources other than $1 \mathrm{~s}_{1 / 2}$ single particle knockout. In fact, the relativistic DWIA calculation indicates that only about $10 \%$ of the strength in the range of $30<E_{\text {miss }}<50 \mathrm{MeV}$ can be accounted for by single particle knockout. This is significantly less than at the lower $P_{m i s s}$ settings, where much of the observed strength in this $E_{\text {miss }}$ range was accounted for by $1 \mathrm{~s}_{1 / 2}$ knockout.

As the figure indicates, the cross section measured at $\theta_{p q}=-16^{\circ}$ has a higher statistical uncertainty than the cross section measured at $\theta_{p q}=+16^{\circ}$. This results from the high level of accidental coincidences present in the $\theta_{p q}=-16^{\circ}$ data due to the close proximity of the hadron spectrometer to the beamline. The subtraction of the high accidental coincidence strength from the total cross section leaves it with a 
large statistical uncertainty.

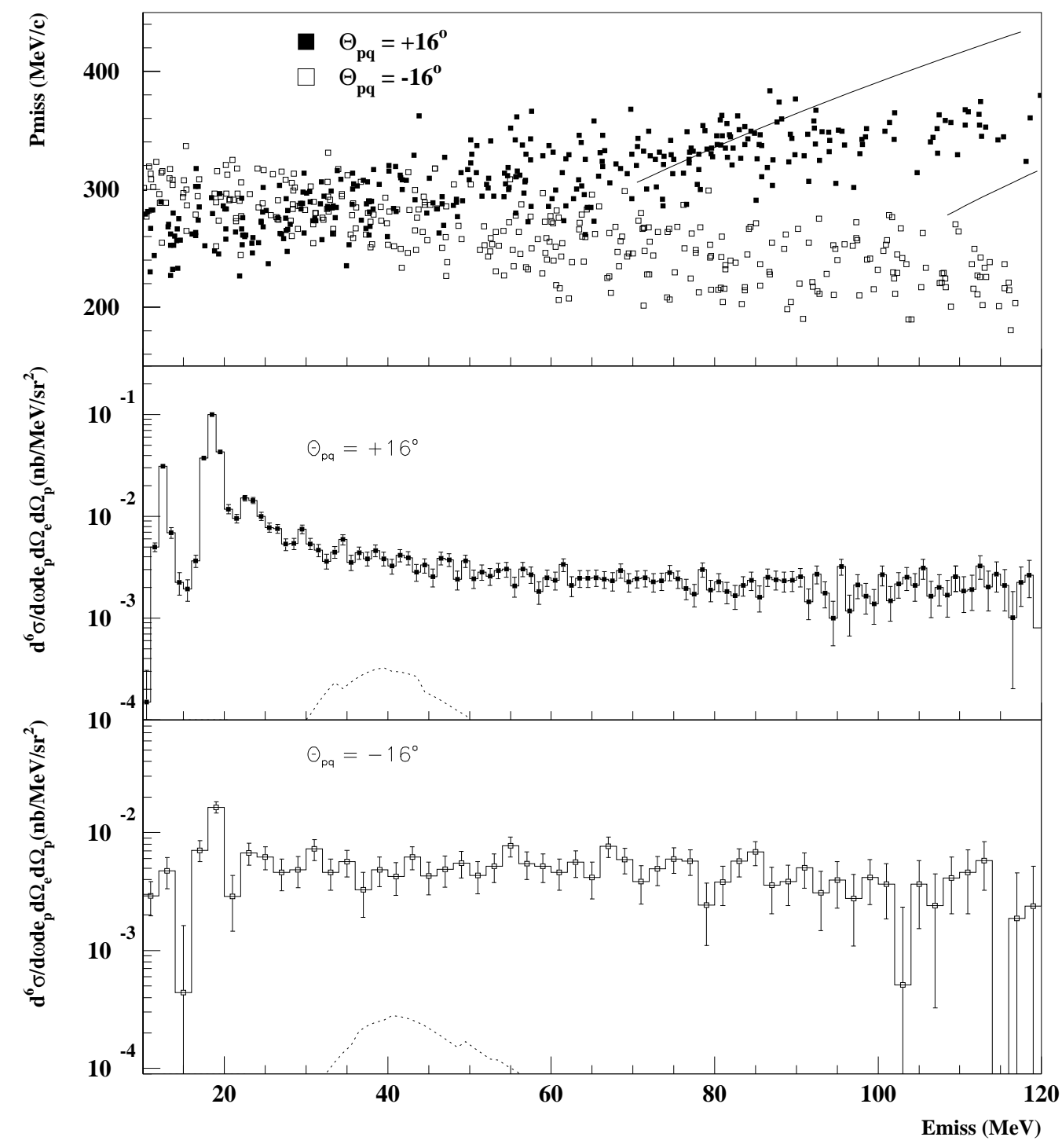

Figure 5-22: radiatively-corrected cross sections for the $E_{0}=2442.3 \mathrm{MeV},\left|\theta_{p q}\right|=16^{\circ}$ kinematic settings. The top plot gives the $\left(E_{\text {miss }}, P_{\text {miss }}\right)$ phase space for the two settings. The two solid lines in the phase space plot indicate the boundaries of the ridge in $\left(E_{\text {miss }}, P_{\text {miss }}\right)$ space that corresponds to the break up of a correlated pair of nucleons. Note that the cross section for the $\theta_{p q}=-16^{\circ}$ setting (bottom plot) is presented using a different binning due to the large statistical uncertainty. The dotted curves indicate the calculation by Van Orden for single particle knockout from the $1 \mathrm{~s}_{1 / 2}$ state. (The spectroscopic factors and the missing energy distributions were obtained as described previously.)

Fig. 5-23 presents the separated response functions for this setting compared to 
the DWIA and HF-RPA calculations. For this setting the total HF-RPA calculation predicts much higher strength for all the response functions than the DWIA calculations. This might be due to the fact that the HF-RPA calculation accounts for long range correlations in the RPA framework while the DWIA calculations do not include the effects of long range correlations.

The longitudinal strength is close to zero as expected by all three calculations. However, the transverse and LT interference response functions are much higher than the DWIA prediction. The total HF-RPA calculation appears to be closer to the data. While the measured $R_{L}$ might appear to follow the $1 \mathrm{~s}_{1 / 2} E_{\text {miss }}$ profile in the range of $30 \mathrm{MeV}<E_{\text {miss }}<50 \mathrm{MeV}$ as predicted by HF-RPA calculations, this not significant statistically. $R_{L T}$, and $R_{T}$ show no clear increase in strength corresponding to the $1 \mathrm{~s}_{1 / 2}$ peak seen at lower $P_{\text {miss }}$.

In both the $\left|\theta_{p q}\right|=16^{\circ}$ and the $\left|\theta_{p q}\right|=8^{\circ}$ settings the HF-RPA calculation appears to reproduce the strength of $R_{L}$ and $R_{T}$ for the $1 \mathrm{~s}_{1 / 2}$ region in $E_{m i s s}$ reasonably well. However, in both cases the HF-RPA calculation underestimates $R_{L T}$ by a significant amount. $R_{L}$ is related to the sum of the squares of the longitudinal components of the hadronic current while $R_{T}$ is related to the sum of the squares of the transverse components. The interference response $R_{L T}$, on the other hand, is related to the relative phase and the amplitudes of the specific parts of the longitudinal and transverse components. The fact that the HF-RPA calculation reproduces $R_{L}$ and $R_{T}$ reasonably well while underestimating $R_{L T}$ might indicate that the specific components of the hadronic current may not be calculated correctly but are compensated for in the sum of the squares.

Fig. 5-24 shows $R_{L T}$ and $R_{T}$ on a logarithmic scale compared to the (e,e'pn) strength calculated by Ryckebusch and to the $1 \mathrm{~s}_{1 / 2}$ single particle knockout strength calculated by Ryckebusch in the Impulse Approximation including MECs and ICs. At this high missing momentum the (e,e'pn) calculation appears to account for about $10 \%$ the transverse strength and for about a few percent of the $L T$ interference strength. The calculated longitudinal strength for (e,e'pn) is close to zero as also indicated by the data. 


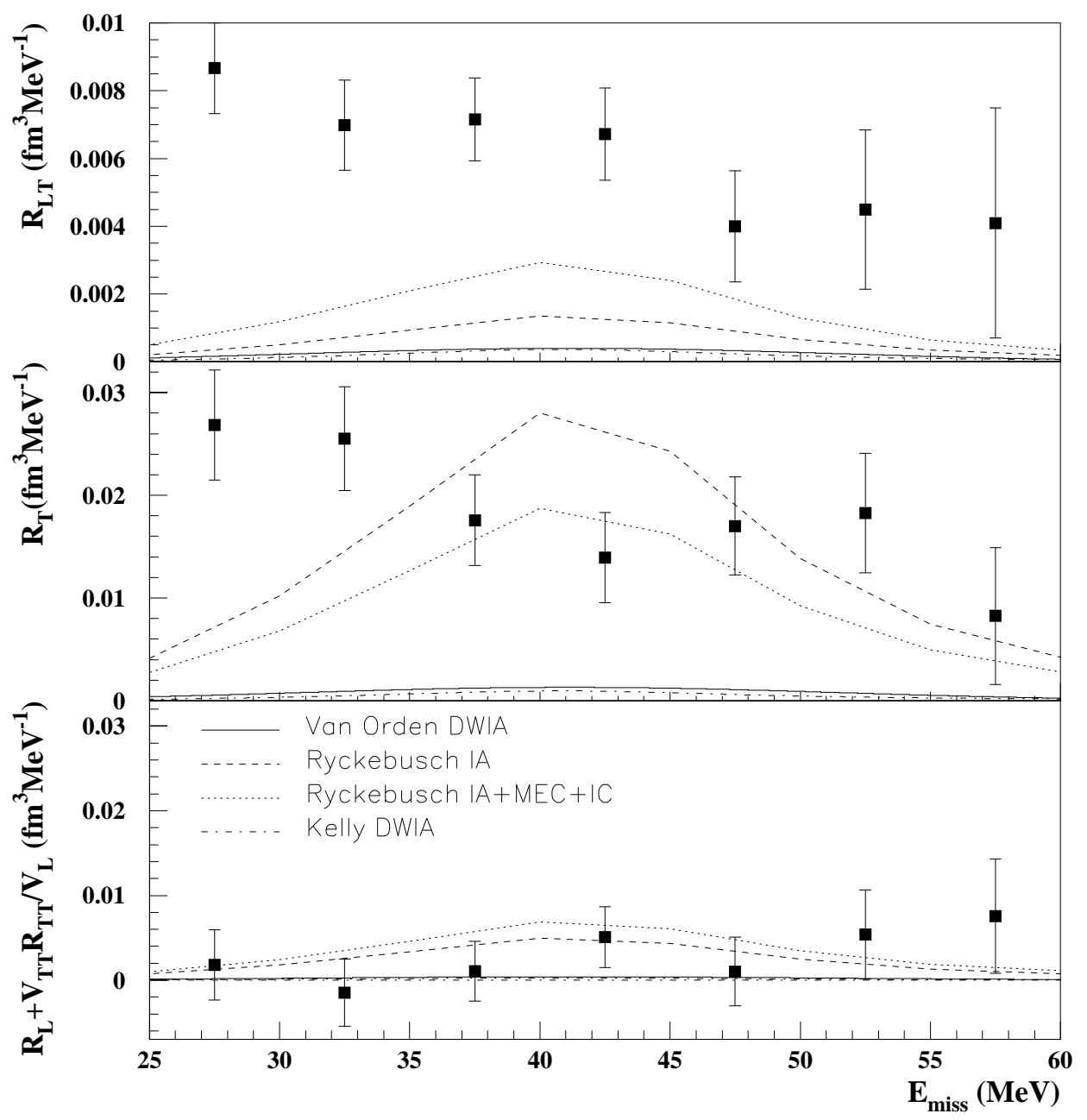

Figure 5-23: Separated response functions for the $\left|\theta_{p q}\right|=16^{\circ}$ setting $\left(P_{\text {miss }}^{\text {average }} \approx 280.0 \mathrm{MeV} / \mathrm{c}\right)$. Plots show (from top to bottom) $R_{L T}, R_{T}$, and $R_{L}+\frac{V_{T T}}{V_{L}} R_{T T}$. The solid curves indicate the calculation by Van Orden for single particle knockout from the $1 \mathrm{~s}_{1 / 2}$ state. The dot dashed curves indicate the calculations by Kelly for the $1 \mathrm{~s}_{1 / 2}$ state. The dashed curve shows the Ryckebusch Impulse Approximation (IA) calculation for $1 \mathrm{~s}_{1 / 2}$ while the dotted curve shows the total IA calculation including Meson Exchange Currents (MEC) and $\Delta$ contributions. 


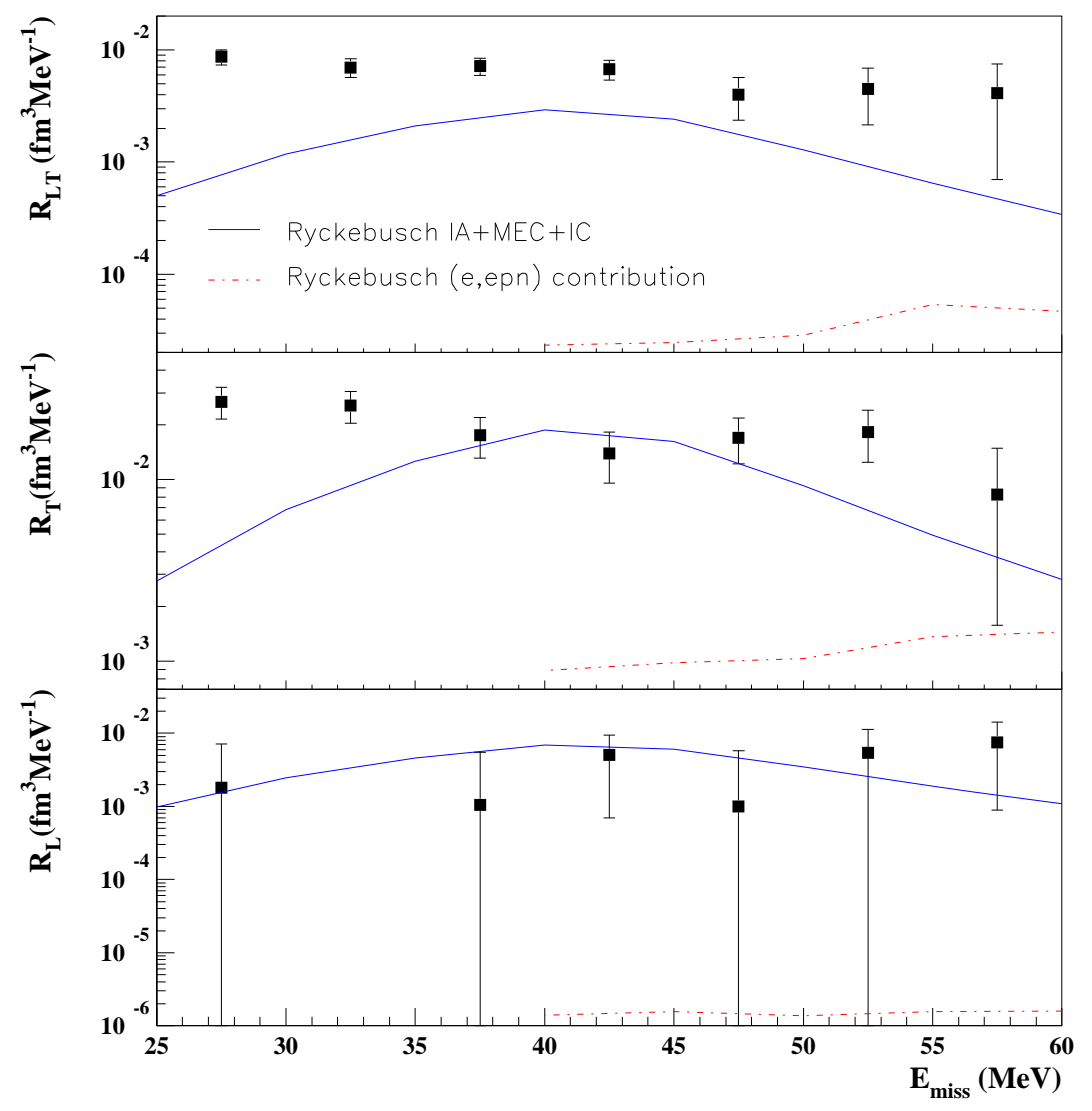

Figure 5-24: Comparison of the calculated response functions for the $\left|\theta_{p q}\right|=16^{\circ}$ kinematics (presented in Fig. 5-23 on a linear scale) to the $1 \mathrm{~s}_{1 / 2}$ strength calculated by Ryckebusch in the Impulse Approximation including MECs and ICs, and to the (e,e'pn) strength calculated by Ryckebusch. (The calculations are preliminary).

\subsubsection{5 $\left|\theta_{p q}\right|=20^{\circ}$ setting $\left(P_{\text {miss }}^{\text {average }} \approx \mathbf{3 4 0 . 0} \mathrm{MeV} / \mathbf{c}\right)$}

Fig. 5-25 presents the radiatively-corrected cross section for the $E_{0}=2442.3 \mathrm{MeV}$, $\left|\theta_{p q}\right|=20^{\circ}$ kinematic settings. No clear increase in cross section is observed at the location of the $1 \mathrm{~s}_{1 / 2}$ shell. The relativistic DWIA calculation indicates that only a very small fraction of the strength in the range of $30<E_{\text {miss }}<50 \mathrm{MeV}$ can be accounted for by single particle knockout.

The two solid lines in the phase space plot indicate the boundaries of the ridge in $\left(E_{m i s s}, P_{m i s s}\right)$ space that corresponds to the break up of a correlated pair of nucleons. The region of $\left(E_{\text {miss }}, P_{\text {miss }}\right)$ phase-space covered by the $\theta_{p q}=+20^{\circ}$ setting falls outside 
this ridge, while the phase space of the $\theta_{p q}=-20^{\circ}$ setting extends across the ridge. The cross section measured at $\theta_{p q}=-20^{\circ}$ shows an increase around the location of the ridge and the cross section measured at $\theta_{p q}=+20^{\circ}$ shows no such increase. This may be a signature of the increase in cross section due to the breakup of a correlated (quasi-deuteron) pair of nucleons. However, it should be noted that there is no clear increase in the cross section around the location of the ridge for the case of $\theta_{p q}=+16^{\circ}$ (see Fig. 5-22).

The lack of matched phase space and the high systematic uncertainty in the cross section prevented the separation of response functions for this $\left|\theta_{p q}\right|$ setting. 


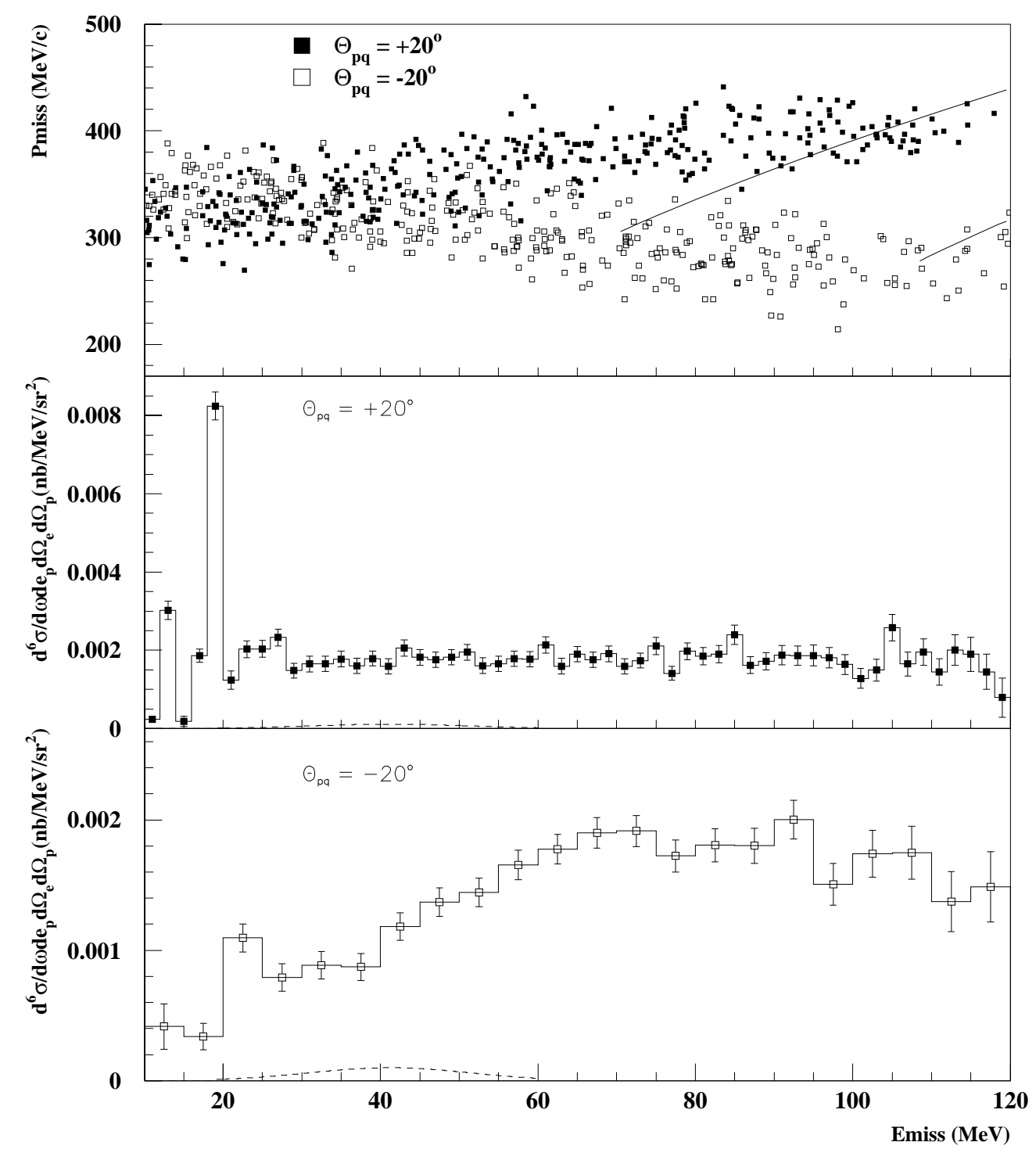

Figure 5-25: radiatively-corrected cross sections for the $E_{0}=2442.3 \mathrm{MeV},\left|\theta_{p q}\right|=20^{\circ}$ kinematic settings. The top plot gives the $\left(E_{m i s s}, P_{\text {miss }}\right)$ phase space for the two settings. The two solid lines in the phase space plot indicate the boundaries of the ridge in $\left(E_{\text {miss }}, P_{\text {miss }}\right)$ space that corresponds to the break up of a correlated pair of nucleons. Note that the cross section for the $\theta_{p q}=-20^{\circ}$ setting (bottom plot) is presented using a different binning due to the large statistical uncertainty. The dashed curves indicate the calculation by Van Orden for the single particle knockout from $1 \mathrm{~s}_{1 / 2}$ state. (The spectroscopic factors and the missing energy distributions were obtained as described previously.)

One underlying feature exhibited by all quasielastic results is that the relative strength in the continuum compared to the strength in the $\mathrm{p}$ shells increases with 
increasing $P_{\text {miss }}$ (see Fig. 5-26). At high $P_{\text {miss }}$, short range and tensor correlations are expected to play an important role. This is especially true for the high $E_{\text {miss }}$ continuum. Such correlations deplete the strength of quasi-hole states and distribute it over a large $E_{\text {miss }}$ range. Muther et al. [47] have calculated the effects of these correlations on ${ }^{16} \mathrm{O}$ using a realistic interaction constructed using a self consistent Green's function approach. They have shown that these correlations have a relatively minor effect in populating high missing momenta of the valence states, while in the high $E_{\text {miss }}$ continuum, a significant strength is shifted towards high missing momenta. The overall effect is the depletion of strength in conventional quasi-hole states to the benefit of high $E_{\text {miss }}$, high $P_{\text {miss }}$ channels.

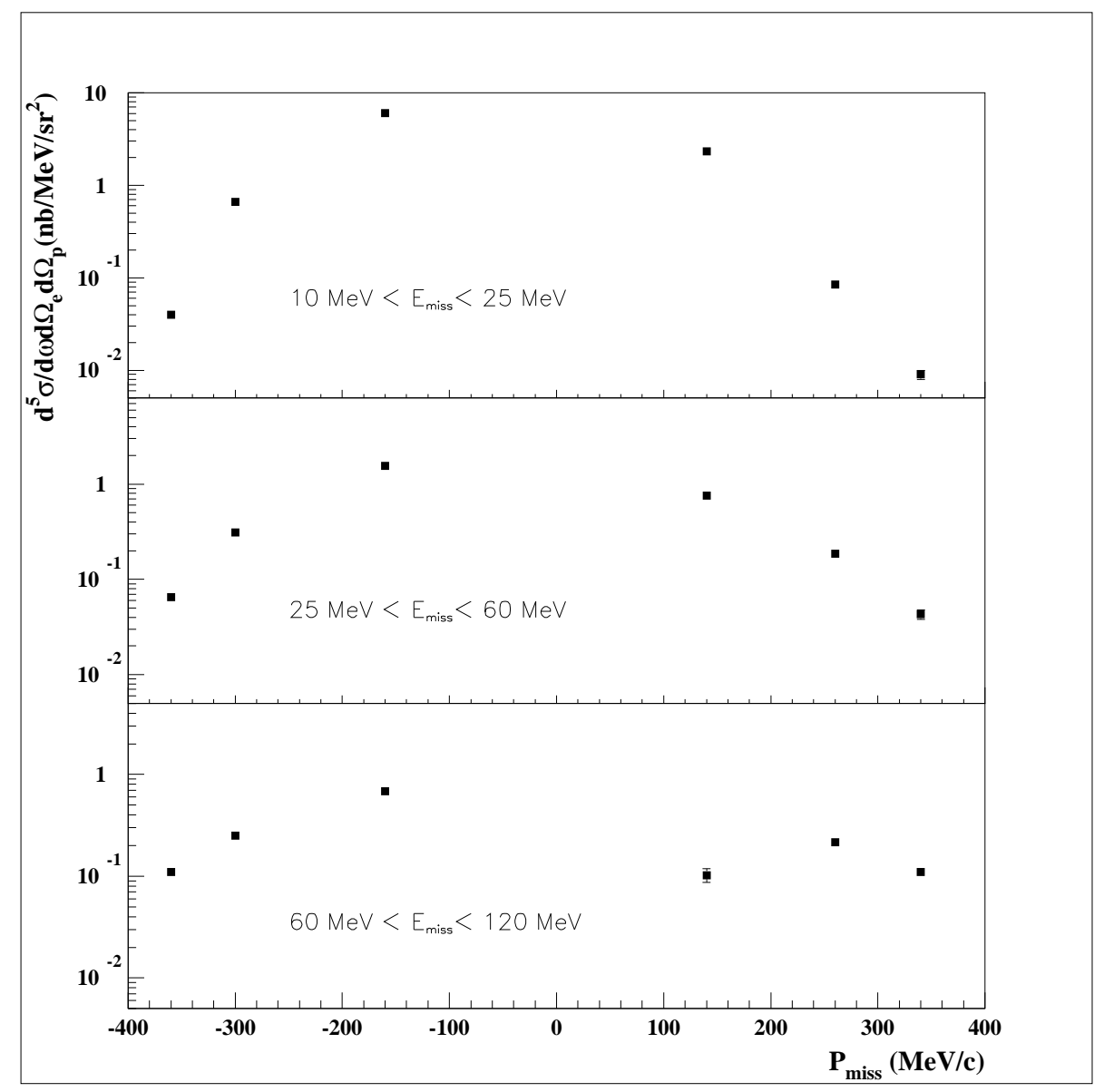

Figure 5-26: Comparison of the cross section integrated over three ranges in $E_{m i s s}$ as a function of missing momentum. At lower $P_{m i s s}$, the cross section is dominated by the p-shell range of $10 \mathrm{MeV}$ $<E_{\text {miss }}<25 \mathrm{MeV}$. At high $P_{\text {miss }}$ the integrated cross section in the deep continuum range of 60 $\mathrm{MeV}<E_{\text {miss }}<120 \mathrm{MeV}$ is much larger than the integrated cross section in the p-shell range. 
A simple model to explain the high $E_{\text {miss }}$, high $P_{\text {miss }}$ strength can be constructed by assuming that this strength arises from the breakup of a correlated pair with a large internal momentum [31], [43]. While one member of the pair is detected in coincidence with the electron, the other member flies off with a large kinetic energy. The kinetic energy of the undetected nucleon results in a large $E_{m i s s}$. To first order $E_{m i s s}$ and $P_{m i s s}$ for this process are (approximately) related by,

$$
E_{m i s s} \approx\left\langle E_{x}^{h h^{\prime}}\right\rangle+\left(\frac{A-2}{A-1}\right) \frac{p_{m i s s}^{2}}{2 M_{p}}
$$

where $M_{p}$ is the proton mass, and $\left\langle E_{x}^{h h^{\prime}}\right\rangle$ is the average excitation energy of the $A-2$ system if the nucleons were knocked-out from orbits characterized by $h$ and $h^{\prime}$.

The solid lines on the phase-space plots in Figs. 5-25 and 5-22 indicate the boundaries of the area for which eqn. 5.13 would predict an increased likelihood to detect ground-state correlation effects. The width of this region was obtained by using 25 $\mathrm{MeV}<\left\langle E_{x}^{h h^{\prime}}\right\rangle<75 \mathrm{MeV}$, thus roughly covering the knockout from $(1 \mathrm{p})^{2},(1 \mathrm{p})(1 \mathrm{~s})$ and $(1 \mathrm{~s})^{2}$ orbitals. A further assumption was made that correlation effects become important for $P_{\text {miss }} \gtrsim 275 \mathrm{MeV}$. Of the kinematic settings covered for this experiment, the $E_{0}=2442.3 \mathrm{MeV}, \theta_{p q}=-20^{\circ}$ setting appears to show an increase in cross section around this area.

A previous ${ }^{16} \mathrm{O}\left(\mathrm{e}, \mathrm{e}^{\prime} \mathrm{p}\right)$ experiment performed at Mainz has shown that for small $P_{m i s s}$, the $E_{m i s s}$ distribution is dominated by valence states, while at high $E_{m i s s}$, it is dominated by the continuum strength. The results from the present experiment exhibit the same pattern.

\subsection{Summary}

In this experiment, the ${ }^{16} \mathrm{O}\left(\mathrm{e}, \mathrm{e}^{\prime} \mathrm{p}\right)$ reaction was studied in the high missing energy range of $E_{m i s s}>20 \mathrm{MeV}$. The ${ }^{16} \mathrm{O}\left(\mathrm{e}, \mathrm{e}^{\prime} \mathrm{p}\right)$ cross section was measured in the dip region at $q=1026 \mathrm{MeV}$ and $\omega=586 \mathrm{MeV}$ for $10 \mathrm{MeV}<E_{\text {miss }}<320 \mathrm{MeV}$. In the quasielastic region, the cross sections were measured for both quasi-parallel kinematics and perpendicular kinematics at $q=1 \mathrm{GeV}$ and $\omega=445 \mathrm{MeV}$. From the data acquired in quasi-parallel kinematics, the longitudinal and transverse response func- 
tions, $R_{L}$ and $R_{T}$ were separated for $E_{\text {miss }}<60 \mathrm{MeV}$. The perpendicular kinematics data were used to extract $R_{L T}, R_{T}$, and $R_{L}+\frac{V_{T T}}{V_{L}} R_{T T}$ response functions for the same $E_{\text {miss }}$ range and for $P_{\text {miss }}<310 \mathrm{MeV}$. This was the first response function separation for the $E_{\text {miss }}$ continuum at high missing momenta ever performed on a complex nucleus.

The results can be summarized as follows:

- In the dip region, the $1 \mathrm{~s}_{1 / 2}$ tail appears to extend to about $E_{m i s s}=90 \mathrm{MeV}$, much further than expected by single-particle mean-field models. This may reflect the increase in importance of the two-body reaction mechanisms in the dip region. After the $1 \mathrm{~s}_{1 / 2}$ tail, the cross section remains at a low, almost flat level up to the $\pi^{0}$ threshold, after which there is a clear increase in the cross section. This might be an indication of the coexistence of at least two different reaction mechanisms at high missing energies in the dip region:

- a mechanism that yields an almost flat continuum strength extending to the very high $E_{m i s s}$ as previously observed at MIT-Bates [41].

- a mechanism connected to $\Delta$-resonant $\pi$ production. Such a mechanism would enhance the cross sections for reactions such as ${ }^{16} \mathrm{O}\left(\mathrm{e}, \mathrm{e}^{\prime} \mathrm{p} \pi^{0}\right)^{15} \mathrm{~N}$. This mechanism was not observed in quasielastic kinematics for similar $|\vec{q}|$ and $\omega[41]$.

- For the quasielastic, quasi-parallel setting, the cross sections and the response functions for $E_{\text {miss }}<50 \mathrm{MeV}$ are mostly accounted for by the single particle knockout from $1 \mathrm{~s}_{1 / 2}$. For $50<E_{\text {miss }}<60, R_{T}$ remains strong while $R_{L}$ appears to go to zero. The difference between the longitudinal and transverse spectral functions, which is expected to be zero for a free nucleon, is close to zero at lower $E_{\text {miss }}$ but appears to increase after the two nucleon emission threshold. This is in qualitative agreement with previous experiments [35]. However, due to the large statistical uncertainty, this is a weak conclusion.

- For the data from the quasielastic, $\left|\theta_{p q}\right|=8^{\circ}$ setting, the behavior of $R_{L}$ and $R_{T}$ is similar to that for the case of quasi-parallel kinematics. In this case, with smaller statistical uncertainties than in the quasi-parallel case, the zero values 
of $R_{L}$ for $E_{\text {miss }}>50 \mathrm{MeV}$ is a stronger conclusion. However, $R_{L T}$ is non-zero for $50 \mathrm{MeV}<E_{\text {miss }}<60 \mathrm{MeV}$, indicating that $R_{L}$ may be small but non-zero in this $E_{\text {miss }}$ range.

- For the quasielastic $\left|\theta_{p q}\right|=16^{\circ}$ setting, the strength in the continuum relative to the $\mathrm{p}$ shell strength is much higher than for the lower $P_{\text {miss }}$ settings.

- The cross sections calculated for the quasielastic $\left|\theta_{p q}\right|=20^{\circ}$ settings show similar behavior to $\left|\theta_{p q}\right|=16^{\circ}$ settings, but with even higher relative strengths in the continuum compared to the valence states. The phase space of the $\theta_{p q}=-20^{\circ}$ setting extends across the two body correlated ridge and shows an increase in cross section around the location of the ridge.

\subsection{Conclusion}

The present results have demonstrated that the relative strength in the continuum compared to the strength in the $\mathrm{p}$ shells increases with increasing $P_{\text {miss }}$. This observation is in qualitative agreement with the theoretical ideas of Muthur et al. [47] and the results from previous experiments. The separated response functions from this experiment at lower $P_{\text {miss }}$ strengthens the previous observation that for $E_{\text {miss }}$ above the $1 \mathrm{~s}_{1 / 2}$ state, the transverse part of the cross section is enhanced. That is, while $R_{L} \rightarrow 0$ for $50 \mathrm{MeV}<E_{\text {miss }}, R_{T}$ remains strong. The present results have shown that at missing momenta above the Fermi momentum, the high excess strength for $25 \mathrm{MeV}<E_{\text {miss }}<60 \mathrm{MeV}$ might be mostly transverse.

The present results have also indicated the increase in the cross section in the high $E_{\text {miss }}$, high $P_{\text {miss }}$ region might be due to the increased likelihood of detecting a member nucleon of a correlated pair in this region. The separated longitudinal and transverse responses of the high $E_{\text {miss }}$, high $P_{\text {miss }}$ cross section would provide a better understanding of the origin of the excess cross section in this region. However, the lack of matched phase space for the present data prevented us from separating the responses in the high $E_{\text {miss }}$, high $P_{\text {miss }}$ region. 


\section{Appendix A}

\section{Calibration of the Hall A High Resolution Spectrometer pair}

The HRS pair was originally commissioned from May '96 to April '97, and the entire commissioning procedure was subsequently repeated for both spectrometers in April '97, prior to this experiment. A 5mm thick tungsten sieve slit with 49 holes was used for the angular calibration. The Hall A event analyzer ESPACE was used to derive optical matrix elements from the commissioning data. These matrix elements allow for the reconstruction of the interaction vertex in the target from the coordinates of the detected particles at the focal plane.

\section{A.1 Coordinate systems}

A detailed description of the coordinate systems used in this document is given in reference [60]. For convenience, a short overview is presented here. All coordinate systems presented are Cartesian. Note that a reference to an angular coordinate in this section should be taken to refer to the tangent of the angle in question.

- Hall Coordinate System (HCS): The origin of the HCS is at the center of the hall, which is defined by the intersection of the electron beam and the vertical symmetry axis of the target system. $\hat{z}$ is along the beam line and points in the direction of the beam dump, and $\hat{y}$ is vertically up. See Fig. A-1. 
- Target Coordinate System (TCS): Each of the two spectrometers has its own TCS. A line perpendicular to the sieve slit surface of the spectrometer and going through the midpoint of the central sieve slit hole defines the $z$ axis of the TCS for a given spectrometer. $\hat{z}_{t g}$ points away from the target. In the ideal case where the spectrometer is pointing directly at the hall center and the sieve slit is perfectly centered on the spectrometer, the $z_{t g}$ axis passes through the hall center. For this case, the distance from the hall center to the midpoint of the central sieve slit hole is defined to be the constant $Z_{0}$ for the spectrometer ${ }^{1}$. The origin of the TCS is defined to be the point on the $z_{t g}$ axis at a distance $Z_{0}$ from the sieve surface. In the ideal case, the origin of the TCS coincides with the hall center. The $x_{t g}$ axis is parallel to the sieve slit surface with $\hat{x}_{t g}$ pointing vertically down. The out-of-plane angle $\left(\theta_{t g}\right)$ and the in-plane angle $\left(\phi_{t g}\right)$ are given by $\frac{d x_{t g}}{Z_{0}}$ and $\frac{d y_{t g}}{Z_{0}}$ respectively. See Fig. A-2.

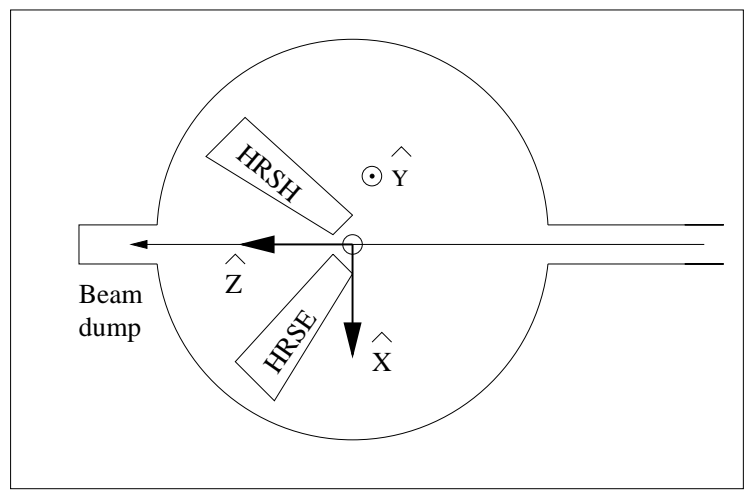

Figure A-1: Hall Coordinate System (top view)

- Detector Coordinate System (DCS): The intersection of wire 184 of the VDC1 U1 plane and the perpendicular projection of wire 184 in the VDC1 V1 plane onto the VDC1 U1 plane defines the origin of the DCS. $\hat{y}$ is parallel to the short symmetry axis of the lower VDC (see Fig. A-3). $\hat{z}$ is perpendicular to the VDC1 U1 plane pointing vertically up, and $\hat{x}$ is along the long symmetry axis of the lower VDC pointing away from the center of curvature of the dipole (see Fig. A-4).

${ }^{1} Z_{0}^{H R S E}=1.181 \mathrm{~m}, Z_{0}^{H R S H}=1.178 \mathrm{~m}$ 


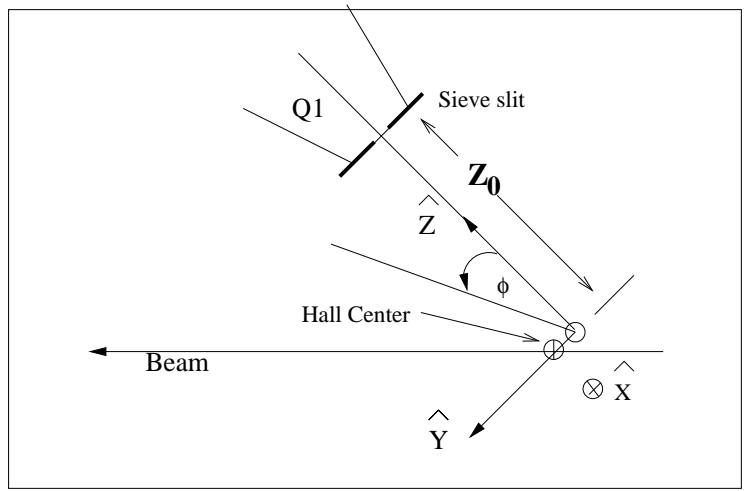

Figure A-2: Target Coordinate System (top view)

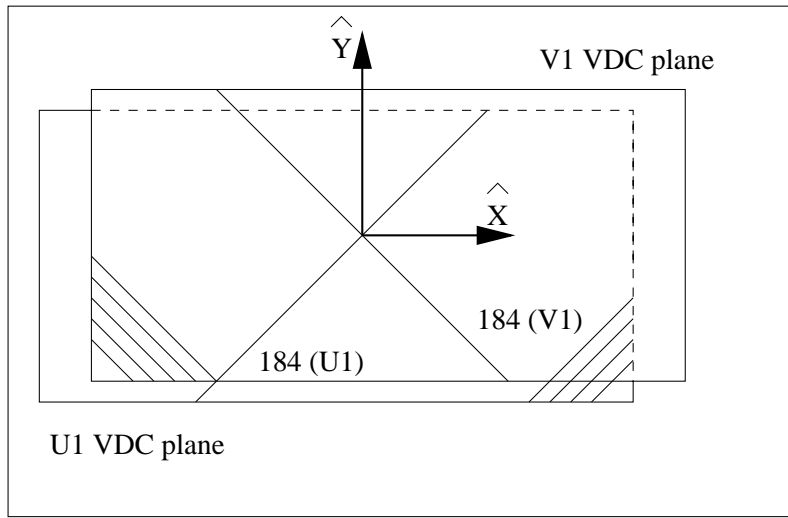

Figure A-3: Detector Coordinate System (top view)

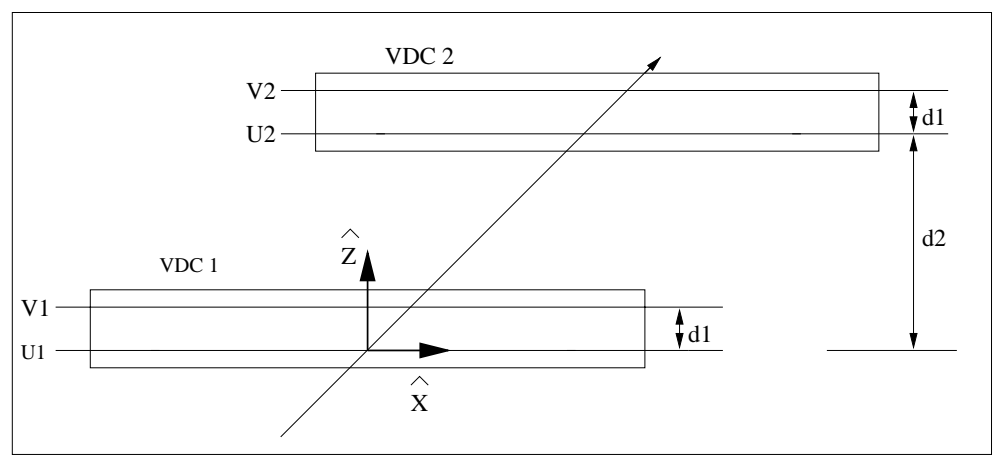

Figure A-4: Detector Coordinate System (side view)

Using the trajectory intersection points $p_{v d c, n}$ (where $n=1-4$ ), with the four VDC 
planes, the coordinates of the detector vertex can be calculated according to

$$
\begin{aligned}
\tan \eta_{1} & =\frac{p_{v d c, 3}-p_{v d c, 1}}{d_{2}}, \\
\tan \eta_{2} & =\frac{p_{v d c, 4}-p_{v d c, 2}}{d_{2}}, \\
\theta_{\text {det }} & =\frac{1}{\sqrt{2}}\left(\tan \eta_{1}+\tan \eta_{2}\right), \\
\phi_{\text {det }} & =\frac{1}{\sqrt{2}}\left(-\tan \eta_{1}+\tan \eta_{2}\right), \\
x_{\text {det }} & =\frac{1}{\sqrt{2}}\left(p_{v d c, 1}+\left(p_{v d c, 2}-d_{1} \tan \eta_{2}\right)\right), \text { and } \\
y_{\text {det }} & =\frac{1}{\sqrt{2}}\left(-p_{v d c, 1}+\left(p_{v d c, 2}-d_{1} \tan \eta_{2}\right)\right)^{\prime}
\end{aligned}
$$

where the distances $d 1$ and $d 2$ are defined in Fig. A-4. These equations may be derived based on the following assumptions:

- the VDC sense wires are oriented at $45^{\circ}$ with respect to the wire frame as shown in Fig. 2-9.

- the wires are positioned in planes.

- the wire planes are parallel to each other and are separated by known distances.

- the location of the center of each wire plane is known.

Any deviation from the above assumptions leads to offsets in the DCS coordinates. These offsets are corrected when the focal plane vertex is calculated.

- Transport Coordinate System (TRCS) at the focal plane: The TRCS at the focal plane is generated by rotating the DCS clockwise around its $y$-axis by $45^{\circ}$. Ideally, the $\hat{z}$ of the TRCS coincides with the central ray of the spectrometer. However, due to the deviations mentioned above, the TRCS used by ESPACE can differ from the ideal spectrometer Transport Coordinate System.

The transport coordinates can be expressed in terms of the detector coordinates 
by

$$
\begin{aligned}
\theta_{t r a} & =\frac{\theta_{d e t}+\tan \rho_{0}}{1-\theta_{d e t} \tan \rho_{0}} \\
\phi_{t r a} & =\frac{\phi_{d e t}}{\cos \rho_{0}-\theta_{d e t} \sin \rho_{0}} \\
x_{t r a} & =x_{d e t} \cos \rho_{0}\left(1+\theta_{\text {tra }} \tan \rho_{0}\right) \\
y_{t r a} & =y_{d e t}+\sin \rho_{0} \phi_{t r a} x_{d e t}
\end{aligned}
$$

where $\rho_{0}$ is the rotation angle, $-45^{\circ}$.

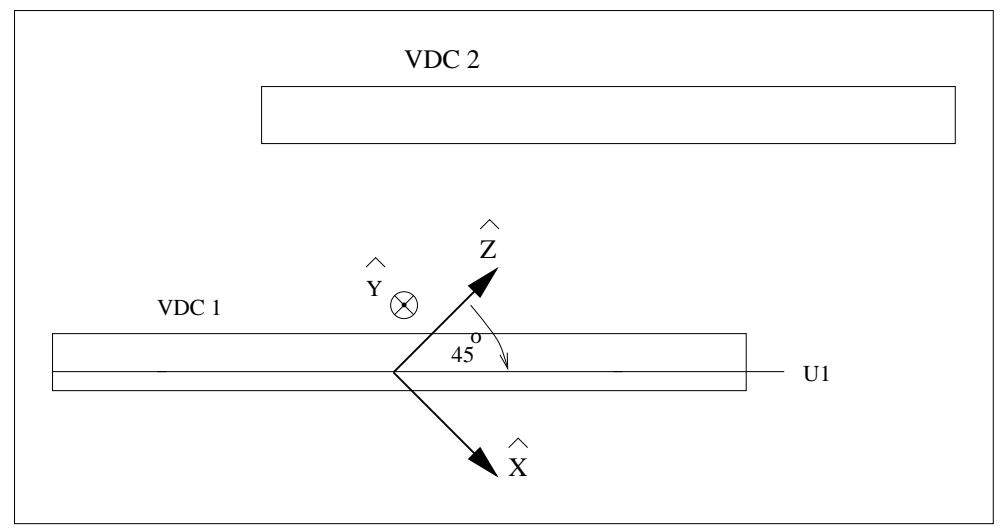

Figure A-5: Transport Coordinate System (side view).

- Focal plane Coordinate System (FCS): The focal plane coordinate system chosen for the HRS analysis is a rotated coordinate system. This coordinate system is obtained by rotating the DCS around its $y$-axis by an angle $\rho$, where $\rho$ is the angle between the local central ray $^{2}$ and the $\hat{z}$ axis of the DCS. As a result, the $\hat{z}$ axis of the FCS rotates as a function of the relative momentum $\frac{\Delta p}{p}$ (see Fig. A-6). In this rotated coordinate system the dispersive angle $\theta$ is small for all points across the focal plane. As a result, the expressions for the reconstructed vertex converge faster during optics calibrations.

\footnotetext{
${ }^{2}$ The ray with $\theta=\phi=0$ for the corresponding relative momentum $\frac{\Delta p}{p}$.
} 


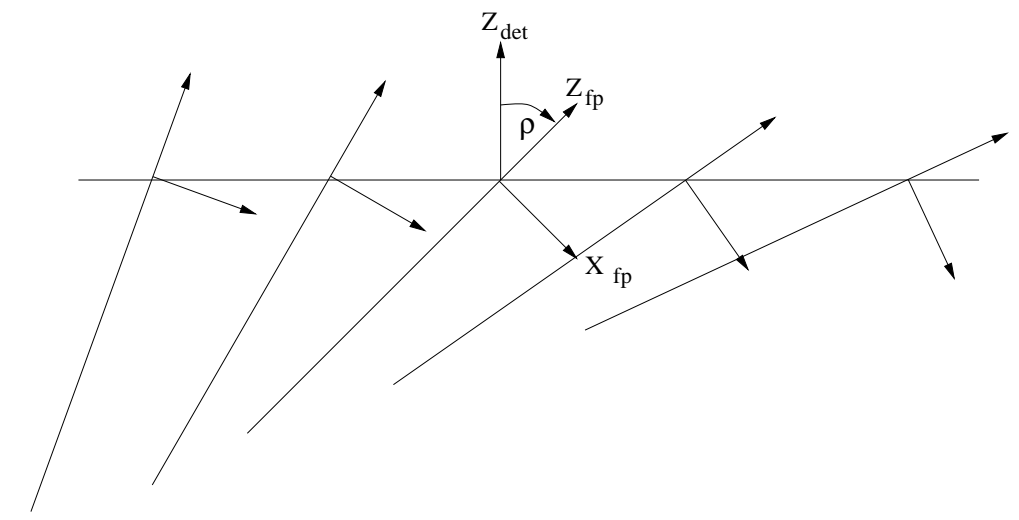

Figure A-6: The focal plane (rotated) coordinate system as a function of the focal plane position.

The transformation to the FCS also includes corrections for the offsets incurred due to misalignments in the VDC package.

The coordinates of focal plane vertex can be written as follows:

$$
\begin{aligned}
y_{f p} & =y_{t r a}-\sum y_{i 000} x_{f p}^{i} \\
x_{f p} & =x_{t r a} \\
\theta_{f p} & =\frac{\theta_{\text {det }}+\tan \rho}{1-\theta_{\text {det }} \tan \rho} \\
\phi_{f p} & =\frac{\phi_{\text {det }}-\sum p_{i 000} x_{f p}^{i}}{\cos \rho-\theta_{\text {det }} \sin \rho},
\end{aligned}
$$

where

$$
\tan \rho=\sum t_{i 000} x_{f p}^{i} .
$$

\section{A.2 Approach}

For each event, two angular coordinates $\left(\theta_{\text {det }}\right.$ and $\left.\phi_{\text {det }}\right)$ and two spatial coordinates $\left(x_{d e t}\right.$ and $\left.y_{d e t}\right)$ are measured at the focal plane. The position of the particle and the tangent of the angle made by its trajectory along the dispersive direction are given by $x_{d e t}$ and $\theta_{\text {det }}$, while $y_{\text {det }}$ and $\phi_{\text {det }}$ give the position and tangent of the angle perpendicular to the dispersive direction. These observables are used to calculate $x$, 
$\theta, y, \phi$, and $\frac{\Delta p}{p} 3$ for the particle at the target. To reduce the number of unknowns at the target to four, the $x_{t g}$ value was effectively fixed at zero during the optics calibration by requiring that the beam position on the target was within $100 \mu \mathrm{m}$ of the origin of the HCS.

The Transport Tensor links the focal plane coordinates to the target coordinates. The relationship between the focal plane and target coordinates can be written (in a first-order approximation) as

$$
\left[\begin{array}{c}
\frac{\Delta p}{p} \\
\theta \\
y \\
\phi
\end{array}\right]_{t g}=\left[\begin{array}{cccc}
\langle\delta \mid x\rangle & \langle\delta \mid \theta\rangle & 0 & 0 \\
\langle\theta \mid x\rangle & \langle\theta \mid \theta\rangle & 0 & 0 \\
0 & 0 & \langle y \mid y\rangle & \langle y \mid \phi\rangle \\
0 & 0 & \langle\phi \mid y\rangle & \langle\phi \mid \phi\rangle
\end{array}\right]\left[\begin{array}{l}
x \\
\theta \\
y \\
\phi
\end{array}\right]_{f p .}
$$

The null tensor elements result from the mid-plane symmetry of the spectrometer.

In practice, the expansion of the focal plane coordinates is performed up to the fifth order. A set of tensors $Y_{j k l}, T_{j k l}, P_{j k l}$ and $D_{j k l}$ link the focal plane coordinates to target coordinates according to ${ }^{4}$

$$
\begin{aligned}
y_{t g} & =\sum_{j, k, l} Y_{j k l} \theta_{f p}^{j} y_{f p}^{k} \phi_{f p}^{l}, \\
\theta_{t g} & =\sum_{j, k, l} T_{j k l} \theta_{f p}^{j} y_{f p}^{k} \phi_{f p}^{l}, \\
\phi_{t g} & =\sum_{j, k, l} P_{j k l} \theta_{f p}^{j} y_{f p}^{k} \phi_{f p}^{l}, \quad \text { and } \\
\frac{\Delta p}{p} & =\sum_{j, k, l} D_{j k l} \theta_{f p}^{j} y_{f p}^{k} \phi_{f p}^{l},
\end{aligned}
$$

where the tensors $Y_{j k l}, T_{j k l}, P_{j k l}$ and $D_{j k l}$ are polynomials in $\mathrm{x}_{f p}$.

\footnotetext{
$3 \frac{\Delta p}{p}=\frac{P-P_{0}}{P_{0}}$, where $P$ is the measured momentum of a particle and $P_{0}$ is the central momentum of the spectrometer.

${ }^{4}$ Note that the superscripts denote the power of each focal plane variable.
} 


$$
\begin{aligned}
Y_{j k l} & =\sum_{i=1}^{m} Y_{j k l, i} x_{f p}^{i} . \\
T_{j k l} & =\sum_{i=1}^{m} T_{j k l, i} x_{f p}^{i} . \\
P_{j k l} & =\sum_{i=1}^{m} P_{j k l, i} x_{f p}^{i} . \\
D_{j k l} & =\sum_{i=1}^{m} D_{j k l, i} x_{f p}^{i} .
\end{aligned}
$$

Mid-plane symmetry of the spectrometer requires that for $Y_{j k l}$ and $P_{j k l},(k+l)$ is odd, while for $D_{j k l}$ and $T_{j k l},(k+l)$ is even.

The transfer tensors are obtained by the minimization of the aberration functions $[98]$

$$
\delta(y)=\sum_{s}\left[\frac{\sum_{j, k, l} Y_{j k l} \theta_{f p}^{j} y_{f p}^{k} \phi_{f p}^{l}-y_{t g}^{0}}{\sigma_{y}^{s}}\right]^{2}
$$

where $\left|y_{t g}^{s}-y_{t g}^{0}\right| \leq w_{y}$

$$
\Delta(\theta, \phi)=\sum_{s}\left[\frac{\sum_{j, k, l} T_{j k l} \theta_{f p}^{j} y_{f p}^{k} \phi_{f p}^{l}-\theta_{t g}^{0}}{\sigma_{\theta}^{s}}\right]^{2}+\sum_{s}\left[\frac{\sum_{j, k, l} P_{j k l} \theta_{f p}^{j} y_{f p}^{k} \phi_{f p}^{l}-\phi_{t g}^{0}}{\sigma_{\phi}^{s}}\right]^{2}
$$

where $\left|\theta_{t g}^{s}-\theta_{t g}^{0}\right| \leq w_{\theta}$ and $\left|\phi_{t g}^{s}-\phi_{t g}^{0}\right| \leq w_{\phi}$, and

$$
\Delta(\delta)=\sum_{s}\left[\frac{\sum_{j, k, l} D_{j k l} \theta_{f p}^{j} y_{f p}^{k} \phi_{f p}^{l}-\delta^{0}}{\sigma_{p}^{s}}\right]^{2}
$$

where $\left|\delta^{s}-\delta^{0}\right| \leq w_{p}$

The index $s$ is varied over all particles detected with their reconstructed vertex $\left(y_{t g}^{s}, \theta_{t g}^{s}, \phi_{t g}^{s}, \delta^{s}\right)$ within the interval $w$ of the peak value $\left(y_{t g}^{0}, \theta_{t g}^{0}, \phi_{t g}^{0}, \delta^{0}\right)$. The contribution of each particle track to the aberration function is weighted by the standard deviation $\left(\sigma_{y}, \sigma_{\theta}, \sigma_{\phi}, \sigma_{\delta}\right)$ calculated for the particular track. The values 
$y_{0}, \theta_{0}, \phi_{0}$ come from surveys.

In practice, the optimization of the transport tensor elements is performed as follows:

- the tensor elements are determined in three steps in the following order: first $Y_{j k l}$, then $P_{j k l}$ and $T_{j k l}$, and finally $D_{j k l}$. Note that the optimization of $Y_{j k l}$ is included here to illustrate the optimization procedure.

- data are acquired for a well-defined, localized $y_{t g}$ point denoted by $y_{t g}^{0}$.

- a set of startup tensor elements, $Y_{j k l}^{i n i t}$, is used with Eqn. A.17 to reconstruct an initial $y_{t g}$ position for each particle according to

$$
y_{t g}^{i n i t}=\sum Y_{j k l}^{i n i t} \theta_{f p}^{j} y_{f p}^{k} \phi_{f p}^{l},
$$

where $y_{f p}, \theta_{f p}$, and $\phi_{f p}$ are given by Eqns. A.11 - A.14 respectively.

- the centroid of the $y_{t g}^{\text {init }}$ distribution (denoted by $y_{t g}^{\text {init } t_{0}}$ ) is calculated for the entire data set.

- the events having $y_{t g}^{\text {init }}$ within a given distance $\left(w_{y}\right)$ of the centroid are selected for the optimization of $Y_{j k l}$ by minimizing the aberration function given in Eqn. A.25.

- tensor elements with principal values smaller than the errors are set to zero. 


\section{A.3 Experimental procedure}

A general set of tensors describing the entire $y_{t g}, \theta_{t g}, \phi_{t g}$ and $\frac{d P}{P}$ space may be obtained by acquiring data that covers a wide range of these variables. This was achieved in practice by performing the following series of calibration experiments:

- at a nominal incident energy of $845 \mathrm{MeV}$, electrons were scattered from a thin ${ }^{12} \mathrm{C}$ target.

1. several different localized $y_{t g}$ points were obtained by physically translating the central axis of the spectrometer parallel to itself by a known amount, as shown in Fig. A-7.

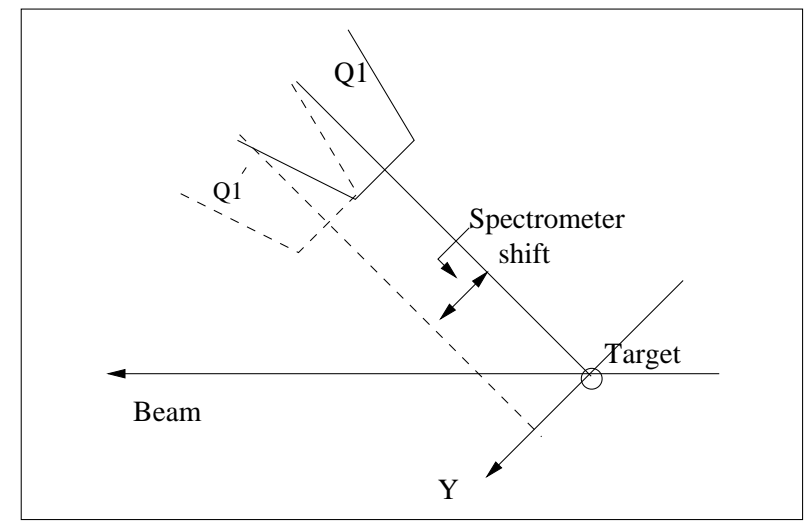

Figure A-7: Spectrometer shift (top view)

2. for each of the $y_{t g}$ points above, seven open collimator measurements were performed at $\frac{d P}{P}$ values varying from $-4.5 \%$ to $4.5 \%$ in steps of $1.5 \%$.

3. all of the above measurements were then repeated with a sieve slit collimator that had 49 holes with well-defined $\phi_{t g}$ and $\theta_{t g}$ values (see Fig. A-8).

- the above measurements were then repeated at an incident energy of $2445 \mathrm{MeV}$. At this energy, a waterfall target with three foils was used [54]. In this case, data from the $\mathrm{H}\left(\mathrm{e}, \mathrm{e}^{\prime}\right)$ reaction was used for the optimization.

During the calibration procedure, the position of the beam on the target was kept to within $100 \mu \mathrm{m}$ of the origin of the HCS. As a result, the intersection point of the beam with the thin target foil provided a point target (to within the spectrometer resolution). The following positions and distances were then surveyed: 
- the target position.

- the spectrometer central angle.

- the displacement of the spectrometer central axis from the hall center.

- the position of the sieve slit center with respect to the spectrometer central axis.

The results from these surveys were used to calculate the $y_{t g}^{0}$ position for each spectrometer setting, and $\phi_{t g}^{0}$ and $\theta_{t g}^{0}$ values for each hole center in the sieve slit.

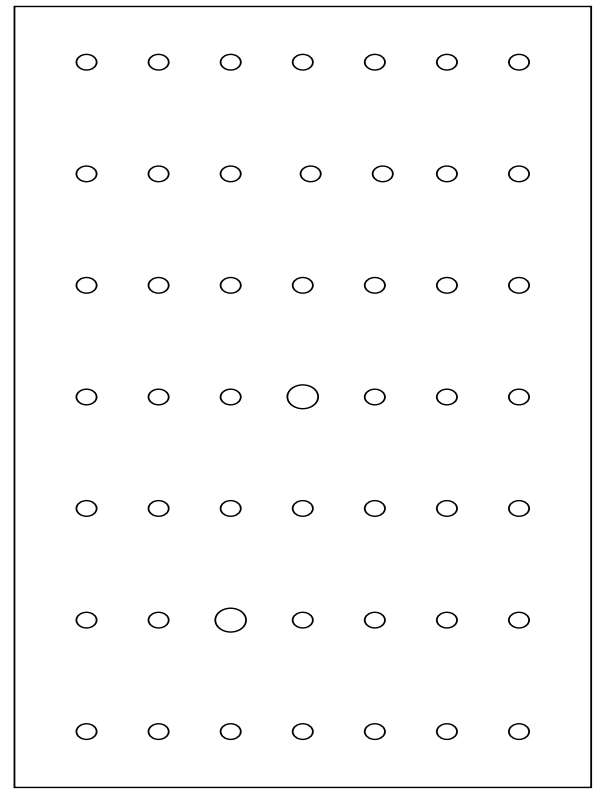

Figure A-8: Sieve slit: The large holes allow for unambiguous identification of the orientation of the image at the focal plane.

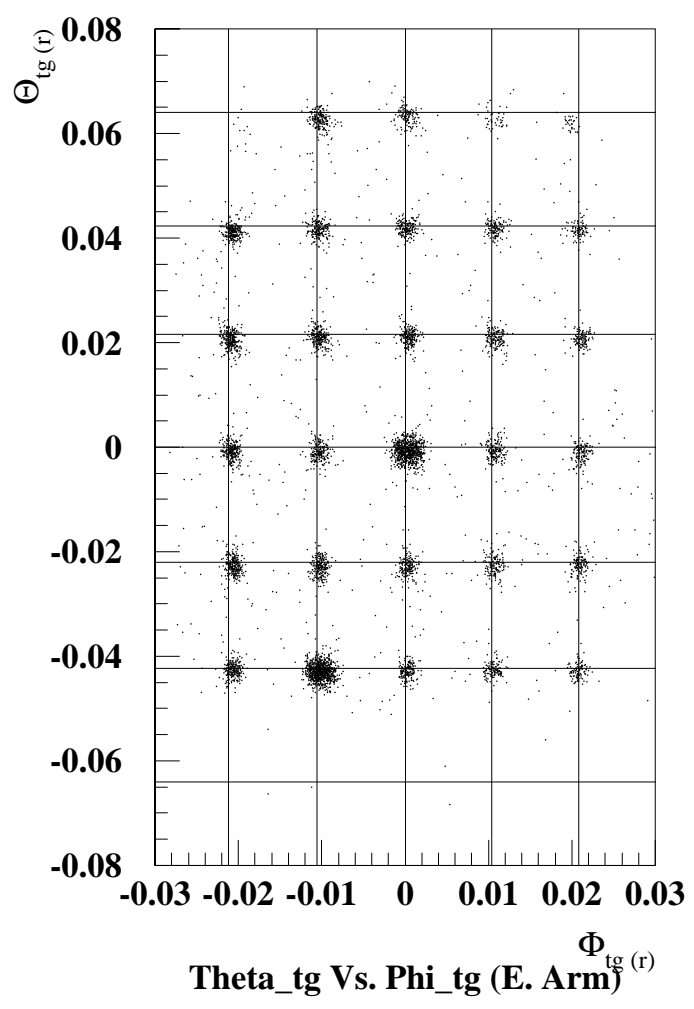

Figure A-9: Reconstructed image of the sieve slit. 


\section{Appendix B}

\section{Basic HRS tensor elements used for E-89003}

See Eqns. A.11 - A.14, A.17 - A.20 and A.21 - A.24 for the definitions of the tensor elements. 


\section{B.1 HRSE}

\begin{tabular}{|l|l|l|l|l|}
\hline Element & $C_{0}$ term & $C_{1}$ term & $C_{2}$ term & $C_{3}$ term \\
\hline \hline t000 & $-1.0027 \mathrm{E}+00$ & $-3.3012 \mathrm{E}-01$ & $-3.2536 \mathrm{E}-02$ & $1.2912 \mathrm{E}-03$ \\
\hline y000 & $-7.2837 \mathrm{E}-03$ & $3.2563 \mathrm{E}-03$ & & \\
\hline p000 & $-2.1774 \mathrm{E}-03$ & $-8.9684 \mathrm{E}-04$ & & \\
\hline D000 & & $8.5175 \mathrm{E}-02$ & $1.0472 \mathrm{E}-02$ & \\
\hline D100 & $-3.5017 \mathrm{E}-02$ & $2.6963 \mathrm{E}-01$ & $4.2004 \mathrm{E}-02$ & $-2.4595 \mathrm{E}-02$ \\
\hline D200 & $-1.4479 \mathrm{E}+00$ & $5.7568 \mathrm{E}-01$ & & \\
\hline D002 & $2.4081 \mathrm{E}-01$ & $-7.7477 \mathrm{E}-02$ & $-6.7727 \mathrm{E}-01$ & \\
\hline D020 & $3.6117 \mathrm{E}-01$ & $-7.5655 \mathrm{E}-01$ & $-7.9962 \mathrm{E}-01$ & \\
\hline D011 & $2.6138 \mathrm{E}-01$ & $6.8103 \mathrm{E}-01$ & & \\
\hline D300 & $2.4742 \mathrm{E}+01$ & & & \\
\hline D120 & $-3.1889 \mathrm{E}+01$ & $1.9336 \mathrm{E}+01$ & & \\
\hline D102 & $-2.6759 \mathrm{E}+01$ & & & \\
\hline D111 & $-1.5159 \mathrm{E}+01$ & $-9.0963 \mathrm{E}+01$ & & \\
\hline
\end{tabular}




\begin{tabular}{|c|c|c|c|c|}
\hline Element & $C_{0}$ term & $C_{1}$ term & $C_{2}$ term & $C_{3}$ term \\
\hline D400 & $1.9030 \mathrm{E}+03$ & & & \\
\hline D202 & $-9.9579 \mathrm{E}+02$ & & & \\
\hline D220 & $-1.6571 \mathrm{E}+03$ & & & \\
\hline D211 & $1.9152 \mathrm{E}+03$ & & & \\
\hline D013 & $-1.4450 \mathrm{E}+02$ & & & \\
\hline $\mathrm{T} 100$ & $-2.2707 \mathrm{E}+00$ & $4.9131 \mathrm{E}-01$ & $1.4908 \mathrm{E}-02$ & \\
\hline $\mathrm{T} 200$ & $-7.2421 \mathrm{E}+00$ & $2.6135 \mathrm{E}+00$ & & \\
\hline T002 & $1.2824 \mathrm{E}-01$ & $4.1137 \mathrm{E}-01$ & $3.6680 \mathrm{E}+00$ & \\
\hline T011 & $1.0730 \mathrm{E}+00$ & $-1.4196 \mathrm{E}+00$ & $-3.7090 \mathrm{E}+00$ & \\
\hline T020 & $-4.2284 \mathrm{E}-01$ & $-3.6365 \mathrm{E}-01$ & & \\
\hline $\mathrm{T} 120$ & $2.5136 \mathrm{E}+01$ & & & \\
\hline $\mathrm{T} 102$ & $2.0016 \mathrm{E}+01$ & $-5.1216 \mathrm{E}+01$ & & \\
\hline T300 & $1.0336 \mathrm{E}+02$ & & & \\
\hline T040 & $5.9637 \mathrm{E}+02$ & & & \\
\hline T022 & $1.0304 \mathrm{E}+03$ & & & \\
\hline $\mathrm{T} 111$ & $-2.1599 \mathrm{E}+01$ & & & \\
\hline $\mathrm{T} 202$ & $-5.4090 \mathrm{E}+02$ & & & \\
\hline T013 & $-1.0237 \mathrm{E}+03$ & & & \\
\hline T031 & $-1.4686 \mathrm{E}+03$ & & & \\
\hline $\mathrm{T} 400$ & $5.0437 \mathrm{E}+03$ & & & \\
\hline P001 & $-6.9215 \mathrm{E}-01$ & $-9.8569 \mathrm{E}-02$ & 2.6903E-01 & -1.0106E-01 \\
\hline $\mathrm{P} 010$ & $-3.2907 \mathrm{E}-01$ & $2.7852 \mathrm{E}-01$ & $-9.4349 \mathrm{E}-02$ & $1.2984 \mathrm{E}-01$ \\
\hline $\mathrm{P} 012$ & $-3.9218 \mathrm{E}+00$ & $5.1591 \mathrm{E}+01$ & & \\
\hline P110 & $3.0497 \mathrm{E}+00$ & $-1.4259 \mathrm{E}-01$ & $-7.7373 \mathrm{E}+00$ & \\
\hline $\mathrm{P} 101$ & $5.9176 \mathrm{E}+00$ & $-7.4720 \mathrm{E}-01$ & $2.6263 \mathrm{E}+00$ & \\
\hline P003 & $1.6310 \mathrm{E}+01$ & $-3.0004 \mathrm{E}+01$ & & \\
\hline P201 & $3.1715 \mathrm{E}+00$ & $-1.5169 \mathrm{E}+02$ & & \\
\hline P210 & $-9.3060 \mathrm{E}+01$ & $7.9745 \mathrm{E}+01$ & & \\
\hline
\end{tabular}




\begin{tabular}{|l|l|l|l|l|}
\hline Element & $C_{0}$ term & $C_{1}$ term & $C_{2}$ term & $C_{3}$ term \\
\hline \hline P030 & $-5.3575 \mathrm{E}+00$ & & & \\
\hline P021 & $-1.7005 \mathrm{E}+01$ & $-1.0125 \mathrm{E}+02$ & & \\
\hline P103 & $-8.3756 \mathrm{E}+02$ & & & \\
\hline P310 & $-1.8746 \mathrm{E}+03$ & & & \\
\hline P112 & $1.2034 \mathrm{E}+03$ & & & \\
\hline P121 & $-7.0797 \mathrm{E}+02$ & & & \\
\hline Y001 & $6.6644 \mathrm{E}-01$ & $-1.2792 \mathrm{E}+00$ & $-5.9088 \mathrm{E}-01$ & $1.0070 \mathrm{E}-01$ \\
\hline Y010 & $-1.1716 \mathrm{E}+00$ & $-7.3591 \mathrm{E}-01$ & $2.0473 \mathrm{E}-01$ & \\
\hline Y110 & $-1.2006 \mathrm{E}+01$ & $-7.4347 \mathrm{E}-01$ & & \\
\hline Y101 & $-5.5873 \mathrm{E}-01$ & $-5.2015 \mathrm{E}+00$ & $-4.7578 \mathrm{E}+00$ & \\
\hline Y012 & $4.9334 \mathrm{E}+00$ & & & \\
\hline Y003 & $2.2622 \mathrm{E}+01$ & & & \\
\hline Y201 & $4.5543 \mathrm{E}+02$ & $2.3879 \mathrm{E}+02$ & & \\
\hline Y210 & $3.1102 \mathrm{E}+02$ & $-9.1130 \mathrm{E}+01$ & & \\
\hline Y030 & $4.7275 \mathrm{E}+01$ & & & \\
\hline Y021 & $6.1093 \mathrm{E}+01$ & $1.8791 \mathrm{E}+02$ & & \\
\hline Y103 & $-7.9386 \mathrm{E}+02$ & & & \\
\hline Y130 & $1.0332 \mathrm{E}+03$ & & & \\
\hline Y112 & $1.8212 \mathrm{E}+03$ & & & \\
\hline Y301 & $3.4467 \mathrm{E}+03$ & & & \\
\hline Y310 & $2.0201 \mathrm{E}+03$ & & & \\
\hline Y121 & $-2.0668 \mathrm{E}+03$ & & & \\
\hline & & & & \\
\hline
\end{tabular}




\section{B.2 HRSH}

\begin{tabular}{|c|c|c|c|c|}
\hline Element & $C_{0}$ term & $C_{1}$ term & $C_{2}$ term & $C_{3}$ term \\
\hline t000 & $-1.0050 \mathrm{E}+00$ & $-3.3613 \mathrm{E}-01$ & $-4.0828 \mathrm{E}-02$ & 4.1021E-03 \\
\hline y000 & $-2.8496 \mathrm{E}-03$ & $-2.0963 \mathrm{E}-03$ & & \\
\hline p000 & $-1.5000 \mathrm{E}-03$ & 3.7015E-03 & $5.4167 \mathrm{E}-04$ & $-3.8885 \mathrm{E}-02$ \\
\hline D000 & & 8.4083E-02 & $1.0977 \mathrm{E}-02$ & \\
\hline D100 & $-3.6552 \mathrm{E}-02$ & 2.8788E-01 & $4.5414 \mathrm{E}-02$ & $-2.4305 \mathrm{E}-01$ \\
\hline D200 & $-1.8889 \mathrm{E}+00$ & $2.3637 \mathrm{E}-01$ & $4.2717 \mathrm{E}+00$ & \\
\hline D002 & $6.5985 \mathrm{E}-03$ & $3.4792 \mathrm{E}-01$ & $-7.3869 \mathrm{E}-01$ & \\
\hline D020 & $5.7923 \mathrm{E}-01$ & $6.7117 \mathrm{E}-01$ & $3.6213 \mathrm{E}-01$ & \\
\hline D011 & $4.7218 \mathrm{E}-01$ & 2.4993E-02 & $-5.0836 \mathrm{E}-01$ & \\
\hline D300 & $3.0423 \mathrm{E}+01$ & $-4.5712 \mathrm{E}+01$ & & \\
\hline D120 & $-6.6381 \mathrm{E}+00$ & $5.4344 \mathrm{E}+01$ & & \\
\hline D102 & $-2.0548 \mathrm{E}+01$ & $-8.2423 \mathrm{E}+00$ & & \\
\hline D111 & $-2.3487 \mathrm{E}+01$ & $-3.6214 \mathrm{E}+01$ & & \\
\hline D400 & $2.1520 \mathrm{E}+03$ & & & \\
\hline D202 & $-3.8587 \mathrm{E}+02$ & & & \\
\hline $\mathrm{D} 220$ & $-4.8900 \mathrm{E}+02$ & & & \\
\hline D004 & $-3.2195 \mathrm{E}+01$ & & & \\
\hline D022 & $-1.5905 \mathrm{E}+02$ & & & \\
\hline D013 & $9.8379 \mathrm{E}+01$ & & & \\
\hline $\mathrm{T} 100$ & $-2.2896 \mathrm{E}+00$ & $5.0418 \mathrm{E}-01$ & $3.5689 \mathrm{E}-02$ & $-7.0449 \mathrm{E}-01$ \\
\hline $\mathrm{T} 200$ & $-3.6320 \mathrm{E}+00$ & $1.0594 \mathrm{E}+00$ & $-1.2168 \mathrm{E}+00$ & \\
\hline T002 & $9.2892 \mathrm{E}-01$ & $-9.9527 \mathrm{E}-01$ & $5.6382 \mathrm{E}-01$ & \\
\hline T011 & $1.0931 \mathrm{E}+00$ & 2.3203E-01 & $-2.2600 \mathrm{E}+00$ & \\
\hline T020 & $1.9557 \mathrm{E}-01$ & $-5.5917 \mathrm{E}-01$ & $1.8563 \mathrm{E}+00$ & \\
\hline $\mathrm{T} 120$ & $-3.5913 \mathrm{E}+01$ & $-5.0596 \mathrm{E}+01$ & & \\
\hline $\mathrm{T} 102$ & $7.0259 \mathrm{E}+00$ & $-1.1836 \mathrm{E}+01$ & & \\
\hline
\end{tabular}




\begin{tabular}{|c|c|c|c|c|}
\hline Element & $C_{0}$ term & $C_{1}$ term & $C_{2}$ term & $C_{3}$ term \\
\hline T300 & $2.6225 \mathrm{E}+01$ & $2.0447 \mathrm{E}+02$ & & \\
\hline Т040 & $4.3965 \mathrm{E}+02$ & & & \\
\hline $\mathrm{T} 220$ & $-2.6534 \mathrm{E}+03$ & & & \\
\hline T022 & $6.7276 \mathrm{E}+02$ & & & \\
\hline $\mathrm{T} 111$ & $-1.6025 \mathrm{E}+01$ & $1.0197 \mathrm{E}+01$ & & \\
\hline $\mathrm{T} 211$ & $-1.4569 \mathrm{E}+03$ & & & \\
\hline T004 & $-4.9292 \mathrm{E}+02$ & & & \\
\hline $\mathrm{T} 202$ & $4.1458 \mathrm{E}+02$ & & & \\
\hline T013 & $-9.8358 \mathrm{E}+02$ & & & \\
\hline T031 & $-8.3672 \mathrm{E}+02$ & & & \\
\hline $\mathrm{T} 400$ & $-3.2178 \mathrm{E}+03$ & & & \\
\hline P001 & $-6.3921 \mathrm{E}-01$ & $-1.3752 \mathrm{E}-01$ & $2.5155 \mathrm{E}-01$ & $3.3808 \mathrm{E}-02$ \\
\hline P010 & -2.6977E-01 & $3.5996 \mathrm{E}-01$ & $-2.9549 \mathrm{E}-01$ & $-4.1009 \mathrm{E}-01$ \\
\hline $\mathrm{P} 012$ & $-2.7086 \mathrm{E}+01$ & $9.6591 \mathrm{E}+01$ & $6.8284 \mathrm{E}+01$ & \\
\hline $\mathrm{P} 110$ & $4.1184 \mathrm{E}+00$ & $-1.4942 \mathrm{E}+00$ & $-3.6635 \mathrm{E}+00$ & \\
\hline P101 & $4.7979 \mathrm{E}+00$ & $1.0219 \mathrm{E}+00$ & $6.6895 \mathrm{E}+00$ & \\
\hline P003 & $2.2081 \mathrm{E}+01$ & $-1.7388 \mathrm{E}+01$ & & \\
\hline P201 & $-4.4012 \mathrm{E}+01$ & $-1.1585 \mathrm{E}+02$ & & \\
\hline P210 & $-8.0492 \mathrm{E}+01$ & $4.5335 \mathrm{E}+01$ & & \\
\hline P030 & $-5.2006 \mathrm{E}+01$ & $-6.8045 \mathrm{E}+01$ & & \\
\hline P021 & $7.2137 \mathrm{E}+01$ & $7.0909 \mathrm{E}+01$ & & \\
\hline P103 & $-8.0018 \mathrm{E}+02$ & & & \\
\hline $\mathrm{P} 130$ & $-9.5414 \mathrm{E}+02$ & & & \\
\hline P301 & $-5.7890 \mathrm{E}+02$ & & & \\
\hline P310 & $-1.2265 \mathrm{E}+03$ & & & \\
\hline $\mathrm{P} 112$ & $1.6526 \mathrm{E}+03$ & & & \\
\hline $\mathrm{P} 121$ & $4.2314 \mathrm{E}+02$ & & & \\
\hline Y000 & $-2.8000 \mathrm{E}-03$ & $5.0000 \mathrm{E}-03$ & & \\
\hline Y001 & $7.2375 \mathrm{E}-01$ & $-1.2442 \mathrm{E}+00$ & $-6.1419 \mathrm{E}-01$ & $-5.1404 \mathrm{E}-02$ \\
\hline
\end{tabular}




\begin{tabular}{|c|l|l|l|l|}
\hline Element & $C_{0}$ term & $C_{1}$ term & $C_{2}$ term & $C_{3}$ term \\
\hline \hline Y010 & $-1.3038 \mathrm{E}+00$ & $-7.2763 \mathrm{E}-01$ & $1.9965 \mathrm{E}-01$ & $2.8169 \mathrm{E}-01$ \\
\hline Y110 & $-1.4394 \mathrm{E}+01$ & $-5.8599 \mathrm{E}-01$ & $-2.1977 \mathrm{E}+00$ & \\
\hline Y101 & $-2.6824 \mathrm{E}+00$ & $-7.7489 \mathrm{E}+00$ & & \\
\hline Y012 & $1.2734 \mathrm{E}+02$ & $-1.4658 \mathrm{E}+02$ & & \\
\hline Y003 & $-2.7597 \mathrm{E}+01$ & $1.5418 \mathrm{E}+02$ & & \\
\hline Y201 & $4.4010 \mathrm{E}+02$ & & & \\
\hline Y210 & $2.3204 \mathrm{E}+02$ & $-1.4032 \mathrm{E}+02$ & & \\
\hline Y030 & $1.0307 \mathrm{E}+02$ & $6.0966 \mathrm{E}+01$ & & \\
\hline Y021 & $-9.2602 \mathrm{E}+01$ & $4.1490 \mathrm{E}+01$ & & \\
\hline Y103 & $7.4580 \mathrm{E}+02$ & & & \\
\hline Y112 & $-8.2829 \mathrm{E}+02$ & & & \\
\hline Y301 & $6.7646 \mathrm{E}+03$ & & & \\
\hline Y310 & $4.2477 \mathrm{E}+03$ & & & \\
\hline
\end{tabular}


Appendix C

The trigger electronics layout 

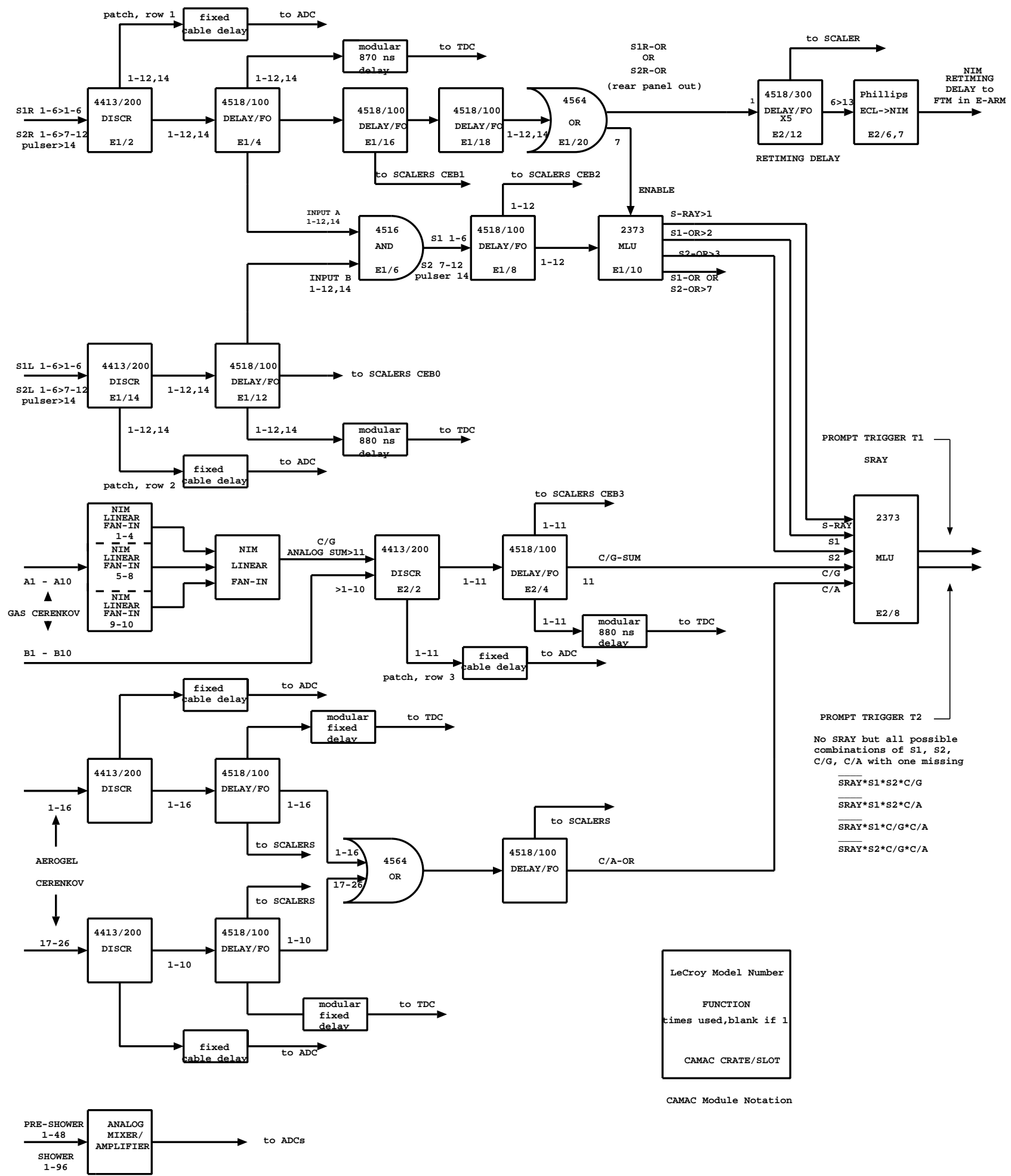

HALI A ELECTRON PROMPT TRIGGER DESIGN 11/13/97

Figure C-1: A schematic diagram of the electron arm trigger electronics 


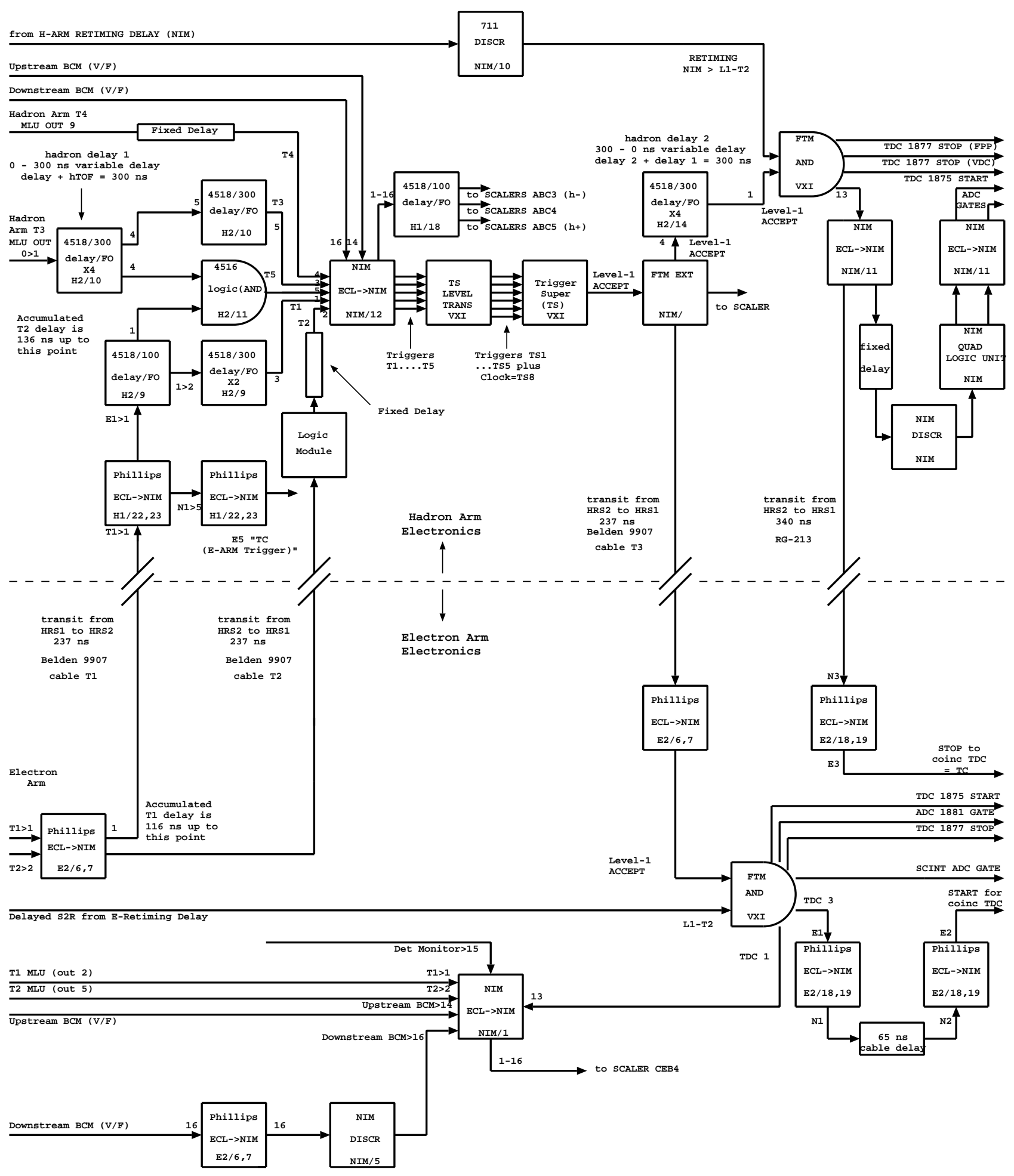

Coincidence Trigger 11/14/97

Figure C-2: A schematic diagram of the coincidence trigger electronics 


\section{Bibliography}

[1] J. Gao, Ph.D. thesis, MIT (1999).

[2] E.J. Moniz, Phys. Rev. 184, 1154 (1969).

[3] P.D. Zimmerman, Ph.D. thesis, Stanford (1969).

[4] R.R. Whitney et al., Phys. Rev. C 9, 2230 (1974).

[5] Z.E. Meziani, et al., Phys. Rev. Lett. 52, 2130 (1984).

[6] Z.E. Meziani, et al., Phys. Rev. Lett. 54, 1233 (1985).

[7] M. Deady et al., Phys. Rev. C 33, 1897 (1986).

[8] W. Alberico et al., Nucl. Phys. A 379, 429 (1982).

[9] W. Alberico et al., Nucl. Phys. A 462, 269 (1987).

[10] G.D. Dang and N.V. Gai Phys. Rev. C 30, 731 (1984).

[11] S. Nishizaki et al., Phys. Lett. B 171, 1 (1986).

[12] L. Clenza et al., Phys. Rev. Lett. 53, 892 (1984).

[13] L. Clenza et al., Phys. Rev. C 31, 946 (1985).

[14] L. Clenza et al., Phys. Rev. C 33, 1012 (1986).

[15] J.V. Noble, Phys. Rev. Lett. 46, 412 (1981).

[16] J.V. Noble, Phys. Lett. B 178, 285 (1986).

[17] P.J. Mulders, Phys. Rev. Lett. 54, 2560 (1985).

[18] J.W. Van Orden et al., Phys. Rev. C 40, 790 (1989). 
[19] W. Alberico et al., Phys. Rev. C 38, 1801 (1988).

[20] T.W. Donnelly and Ingo Sick, MIT-CTP Report 2780 (LANL pre-print nuclth/9809063).

[21] T.W. Donnelly et al., MIT-CTP Report 2722 (LANL pre-print nucl-th/9804054).

[22] J. Jourdan, Nucl. Phys. A 603, 117 (1996).

[23] T. C. Yates et al., Phys. Lett. B312, 382 (1993).

[24] C. F. Williamson et al., Phys. Rev. C 56, 3152 (1997).

[25] T.W. Donnelly, Progress in particle and Nuclear Physics, 183 (1985).

[26] T.W. Donnelly and A.S. Raskin, Ann. Phys 169, 247 (1986).

[27] T.W. Donnelly and A.S. Raskin, Ann. Phys 191, 78 (1989).

[28] A. Picklesimer et al. Phys. Rev. C 32, 1312 (1985).

[29] A. Picklesimer and J.W. Van Orden Phys. Rev. C 35, 266 (1987).

[30] S. Boffi et al., Nucl. Phys. A 476, 617 (1988).

[31] J.J. Kelly, Adv. Nucl. Phys., 23 ed. by J.W. Negele and E. Vogt 75 (1996).

[32] T. de Forest, Nucl. Phys. A 392, 232 (1983).

[33] T. de Forest, Ann. Phys 45, 365 (1967).

[34] S. Frullani and J. Mougey, Adv. Nucl. Phys., 14 edited by J.W. Negele and E. Vogt 75 (1984).

[35] P.E. Ulmer et al., Phys. Rev. Lett. 59, 2259 (1987).

[36] P.E. Ulmer, Ph.D. thesis, MIT (1998).

[37] R. Lourie et al., Phys. Rev. Lett. 56, 2364 (1986).

[38] R. Lourie Ph.D. thesis, MIT (1986).

[39] L. Weinstein et al., Phys. Rev. Lett. 64, 1646 (1990). 
[40] L. Weinstein, Ph.D. thesis, MIT (1988).

[41] J.H. Morrison, Ph.D. thesis, MIT (1993).

[42] M.W. Holtrop, Ph.D. thesis, MIT (1995).

[43] J. Ryckebusch et al., Nucl. Phys. A 624, 581 (1997).

[44] J. Ryckebusch et al., Nucl. Phys. A 476, 237 (1988).

[45] J. Ryckebusch et al., Nucl. Phys. A 503, 694 (1989).

[46] M. Waroquier et al., Phys. Rep. 148, 249 (1987).

[47] H. Muther and W.H. Dickhoff et al., Phys. Rev. C 49, R17 (1994).

[48] K. I. Blomqvist et al. Phys. Lett. B 344, 85 (1995).

[49] P. E. Ulmer et al., Beam Charge Measurements for Hall A experiment E89-003 at Jefferson Laboratory (1998)

[50] K. Unser, IEEE Trans. Nucl. Sci. 16, 934 (1969).

[51] K. Unser, IEEE Trans. Nucl. Sci. 28, 2344 (1981).

[52] http://www.cebaf.gov/accel/documents/epics_doc.html.

[53] Jefferson Lab Data Aquisition group, http://alcor.cebaf.gov/coda2.0/manuals/index.html.

[54] F. Garibaldi et al., Nucl. Instrum. Methods A314, 1 (1992).

[55] "Hall A experimental equipment operations manual", ed. by S. Nanda (1997).

[56] K. G. Fissum et al., "Vertical Drift Chambers for the Hall A High Resolution Spectrometers", to be submitted to Nucl. Instrum. Meth. in 1999

[57] C. Leathers B.S. Thesis MIT (1996)

[58] R.H. Wechsler B.S. Thesis MIT (1996)

[59] R. Veenhoff, GARFIELD, a drift chamber simulation program: User's guide. CERN (1995)

[60] E.A.J.M. Offerman, ESPACE users guide (1997). 
[61] Online codes DATASPY and DHIST for Hall A spectrometers", Robert Michaels, http://www.cebaf.gov/Hall-A/equipment/daq/dplot.html

[62] J. Gao et al., Beam energy measurement at Jefferson Lab using the Hall A Spectrometers, MIT-LNS IR \#0498

[63] J.J. Kelly, ALLFIT: lineshape fitting program for nuclear reactions, http://www2.physics.umd.edu/ jjkelly/allfit.html

[64] G.G. Simon et al., Nucl. Phys. A 333, 381 (1980).

[65] D.V. Jordan, Ph.D. thesis, MIT (1994)

[66] E. Quint, Ph.D. thesis, University of Amsterdam (1988).

[67] J. Schwinger, Phys. Rev. 76, 760 (1949).

[68] D.R. Yennie, S. Frautschi, and H. Suura, ann. Phys. 13, 379 (1961).

[69] Y.S. Tsai, Phys. Rev. 122, 1898 (1961).

[70] L.M. Mo and Y.S. Tsai, Rev. Mod. Phys. 41, 205 (1969).

[71] N. Makins, Ph.D. Thesis, MIT (1994).

[72] S. Penner, Nuclear Structure Physics, Proceedings of the $18^{\text {th }}$ Scottish Univ. Summer School in Physics. 284 (1997)

[73] J. Friedrich, Nucl. Inst. and Meth. 129, 505 (1975).

[74] D.J.S. Findlay and A.R. Dusautoy, Nucl. Inst. and Meth. 174, 531 (1980).

[75] H. Crannell, Nucl. Inst. and Meth. 71, 208 (1969).

[76] CERN Program Library Long Writeup Y250, http://wwwinfo.cern.ch/asdoc/ hbook_html3/hboomain.html.

[77] J.P. Jeukenne and C. Mahaux, Nucl. Phys. A 394, 445 (1983).

[78] J.J. Kelly, LEA: Linear Expansion Analysis, A program to compute nucleon-nucleus scattering and nucleon knockout by electron scattering. "http://www.nscp.umd.edu/ kelly/LEA/lea.html" 
[79] J.J. Kelly, Phys. Rev. C 56, 2672 (1997).

[80] M.F. Gari and W. Krümpelmann, Phys. Lett. B 274, 159 (1992).

[81] M.F. Gari and W. Krümpelmann, Phys. Lett. B 282, 483 (1992).

[82] Z. Y. Ma and J. Wambach, Nucl. Phys. A 402, 275 (1983).

[83] Z. Y. Ma and J. Wambach, Phys. Lett. B 256, 1 (1991).

[84] E.D. Cooper et al., Phys. Rev. C 47, 297 (1993).

[85] M. Leushner et al, Phys. Rev. C 49, 955 (1994).

[86] T. Takaki, Phys. Rev. C 39, 359 (1989).

[87] H. Bertini, Phys. Rev. 131, 1801 (1963).

[88] H. Bertini, Phys. Rev. 188, 1711 (1969).

[89] S. Nozawa and T. S. H. Lee Nucl. Phys. A 513, 511 (1990).

[90] A. Picklesimer, J. W. Van Orden, and S. J. Wallace, Phys. Rev. C32, 1312 (1985).

[91] A. Picklesimer and J. W. Van Orden, Phys. Rev. C40, 290 (1989).

[92] Krishni Wijesooriya, Ph.D. thesis, The College of William and Mary (1999).

[93] L. Lapikas, Nucl. Phys. A 553, 297 (1993).

[94] W. Bertozzi, Nucl. Phys. A 527, 347 (1991).

[95] J.B.J.M. Lanen et al., Phys. Rev. Lett. 64, 2250 (1990).

[96] G. van der Steenhoven et al., Nucl. Phys. A 480, 547 (1988).

[97] G. van der Steenhoven et al., Nucl. Phys. A 484, 445 (1988).

[98] E.A.J.M. Offerman, Ph.D thesis (1988). 


\section{Acknowledgments}

First and foremost, I would like to thank my advisor Professor Bill Bertozzi for his guidance, support, enthusiasm and knowledge. Bill shaped my physics career and tried to make a physicist out of me since I first joined his group as an undergraduate research assistant in 1991.

I would like to thank Shalev Gilad. Most graduate students have one advisor, I had two. Shalev taught me how to build good wire chambers and was a source of valuable guidance and advice throughout my time with the Nuclear Interactions Group.

I would like to thank Kevin Fissum for all his hard work to make this experiment a success, for all his help with the data analysis, for taking over the VDC responsibilities so that Juncai and I could concentrate on data analysis, for his tremendous help with all my technical writing, for putting up with my most unreasonable requests for proof-reading, and for his friendship.

I would like to thank Larry Weinstein for all his contributions to this experiment from its conception. Larry was a spokesperson of the experiment. His help with the details of the experimental technique and data analysis were most valuable.

I would like to thank both Larry and Kevin for reading the draft of this thesis and for the valuable comments and suggestions. It is rather unfortunate that I did not have enough time to incorporate some of those suggestions. If I had, that would have improved the quality of this thesis by several orders of magnitude.

I would like to thank Arun Saha for all his efforts to make this experiment happen and for his advice and help throughout the experiment and data analysis. Arun was one of the spokespersons of the experiment as well as the contact person.

I would like to thank Paul Ulmer for his invaluable help during the experiment and the data analysis. Paul's experience with $\left(e, e^{\prime} p\right)$ experiments helped us through many difficult issues during data analysis.

I would like to thank Meme Liang for the invaluable role she played in this experiment, for all her help with the analysis, for her work related to the spectrometer surveys, and most of all for her work with the trigger simulation which helped us 
calculate the dead-time correction factors properly.

I would like to thank Larry Cardman and Kees De Jager for their leadership of Hall $\mathrm{A}$ in the years proceeding this experiment. I would like to thank the Hall A staff members: J. P. Chen, Javier Gomez, John LeRose, Meme Liang, Robert Michaels, Sirish Nanda, Eddy Offerman, Arun Saha and Bogdan Wojtsekhowski for all their commitment and hard work to get the Hall A experimental setup ready for this experiment. I would also like to thank Eugene Chudakov, Ole Hansen and Michael Kuss for their valuable advice during the last several months. I would like to thank the technical staff: Ed Folts, Jack Segal, Mark Stevens, James Proffitt, and Scot Spiegel for their hard work and many sleepless nights which made this experiment possible. I would especially like to thank Bogdan, Jack, and Howard Fenker for all their advice and help during the VDC project.

I would like to thank the members of the Hall A collaboration for their efforts to put together the Hall A experimental setup and for all the shifts during the experiment.

I would like to thank the past and present post-docs of the Nuclear Interactions Group: Dan Dale, Adam Sarty, J. P. Chen, Jeff Templon, Jianguo Zhao, Kevin Fissum, Zilu Zhou, and David Rowntree for their help, advice, support and friendship throughout my many years with the group. I would especially like to thank J. P. and Jeff for their work with the VDCs and David for his work on charge measurements and spectrometer survey software for this experiment.

I would like to thank Professors June Matthews and Earle Lomon for agreeing to serve on my thesis committee at such short notice and for all their valuable comments.

I owe a great deal to Juncai Gao. I was very lucky to have Juncai as my fellow graduate student in the VDC project and in the experiment and as my officemate at the lab. Without Juncai's help it would have taken me many more years to finish the data analysis. I benefited tremendously from his sharp physics knowledge.

I would like to thank Jim Kelly for the most valuable codes LEA and ALLFIT, for the $\left(e, e^{\prime} p\right)$ review, for the calculations, for very quickly providing me with several figures in this thesis and for many physics discussions.

I would also like to thank Jan Ryckebusch and Wally Van Orden who have been most helpful with the calculations. 
I would like to thank Mike Finn for useful physics discussions, for support and for encouragement.

I would like to thank Joanne Gregory for all her help beginning the day I joined the group. I am sure I will miss Joanne. I would like to thank Marjorie Neal for teaching me fine technical skills associated with wire chamber construction. I would like to thank Joanne and Marjorie also for their kindness and support through one of the most difficult periods of my life.

I would like to thank Hall A graduate students and my fellow trailer dwellers who helped make my life away from MIT a little more bearable. They include (but are not limited to): Paul Gueye, Mark Jones, Bill Kahl, Michael Kuss, Sergey Malov, Kathy Mccormick, David Prout, Gilles Quemener, Riad Suleiman, Luminita Tudor, Rob van der Meer, Wenxia Zhao and of course Krishni Wijesooriya.

I would like to thank my fellow Nuclear Interactions Group graduate students (past and present): Maurik Holtrop, Richard Florizone, Jiang Chen, Haijun Song, Zhengwei Chai, Bin Zhang and Marat Rvachev for their friendship. I would especially like to thank Richard for his help with RADCOR.

I would like to thank my friends whose help, support, and kindness sustained me through my difficult times and helped me get through graduate studies without permanent damage. Some names that readily come to my mind are Vipuli Wijetunga and Nalantha Amarasekara, Udaya and Shiroma Liyanage, Charles and Margaret Hallisey, Lotta Ekberg, Jessica Clark, Julie Grossen, and Gina Hoatson. I would also like to thank Ira, Vajee, Kamal and Selaka for their love and support.

I would like to thank my mother Sumana Liyanage for all her love, support and for everything she has done for me. She was the one who encouraged me to become a physicist. When she did that, she must have known that it would require me to be several thousand miles away from her and I know that she did not like it, but she still told me that I would be happier as a physicist than as a medical doctor. I would also like to thank my two brothers Gayan and Anusha for their love.

I would like to thank my son Seth for brightening my days and for being such an easy baby to look after. If he had wanted to he could have made me take many more months to finish this thesis.... 
Finally I would like to thank my wife Krishni for all her love, patience, support, friendship and all the happiness she brings in to my life. If not for Krishni, I would never have come to MIT. During a time of great chaos and uncertainty in my life Krishni told me about MIT (I was so ignorant I did not even know that it existed) and forced me to apply . I would also like to thank Krishni for all her important comments on the thesis, for her help with the analysis of E-89033 data and for showing me how to run LEA. 